\title{
Starvation/stationary phase survival of Rhodococcus erythropolis SQ1: a physiological and genetic analysis
}

\author{
By Nicolas VJ Fanget \\ Dissertation submitted to the Faculty of Health, Life and Social Sciences, \\ Napier University, Edinburgh, in partial fulfilment of the requirements for \\ the degree of Doctor of Philosophy, 2008.
}

Supervisor: Dr Sophie Foley 


\section{Abstract}

Although the starvation or non-growth state is probably the most common physiological state of bacteria, it has been studied in relatively few organisms. In spite of its importance in pathogenesis, bioremediation and several industrial processes, limited research has been performed on Rhodococcus under starvation/stationary phase conditions. The objectives of this study were to analyse the physiological adaptation of Rhodococcus erythropolis SQ1 to starvation/stationary phase, and to generate and screen a bank of mutants to identify genetic elements involved in this adaptation.

It was found that $R$. erythropolis SQ1 can survive for at least 43 days in LB and distilled water, and 65 days in chemically defined medium (CDM) containing high (1\% $\mathrm{w} / \mathrm{v})$ or low $(0.1 \% \mathrm{w} / \mathrm{v})$ glucose concentrations. Early stationary phase $R$. erythropolis SQ1 cells grown in $0.1 \%$ glucose also exhibited enhanced resistance to heat and oxidative stress compared with exponential phase cells. A mutant bank of $898 R$. erythropolis SQ1 mutants was generated and screened; four mutants were of particular interest. The culturability of mutants 4G6 and 10D3 dropped to $<0.1 \%$ of the maximum $\mathrm{CFU} / \mathrm{ml}$ at 27 days incubation, and to $<3 \%$ of the maximum CFU/ml for mutants $1 \mathrm{~B} 2$ and $1 \mathrm{H} 1$, when grown in $1 \%$ glucose medium. No drop in culturability was observed when mutants were grown in $0.1 \%$ glucose. Mutant 4G6 had a transposon insertion in $u v r B$ (UvrB, part of the DNA excision repair mechanism), while the insertion for mutant 10D3 was immediately downstream of a putative guaB gene, which, based on bioinformatic analyses, is followed by another putative IMP dehydrogenase (guaB-like) and/or a cholesterol oxidase gene. In mutant $1 \mathrm{H} 1$ the transposon inserted 272 nucleotides downstream of a gene encoding a putative phosphoglycerate mutase and upstream of putative thioredoxin and cytochrome $c$ biogenesis genes.

In conclusion, $R$. erythropolis SQ1 was shown to present a classic starvation/stationary phase survival response, with the associated increase in resistance to various external stresses. A mutant bank has been generated which can be used in the future to analyse other phenotypes of interest. Several genes linked to starvation/stationary phase survival were identified. These findings show that a wide variety of genes are involved in starvation/stationary phase survival. Indeed, over 100 such genes have been identified in Escherichia coli and Mycobacterium tuberculosis. 


\section{Acknowledgements}

This thesis would never have been competed without the enduring support of my parents and my dearest wife, Amina. I also wish to thank my Supervisor Dr Sophie Foley for her patience and help, and all the people I worked with at Napier University. 


\section{Table of contents}

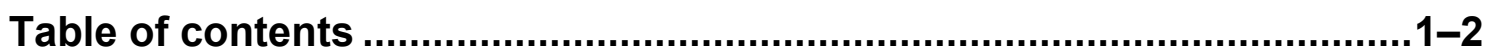

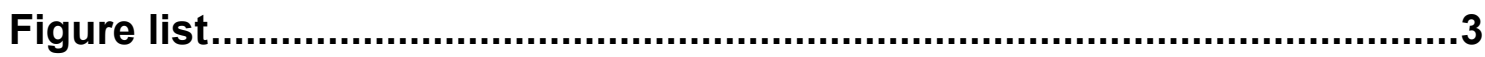

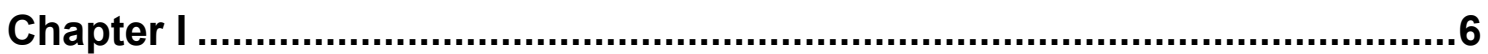

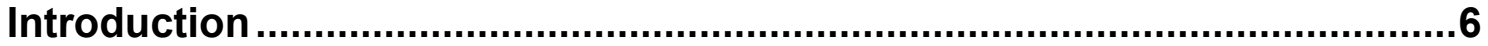

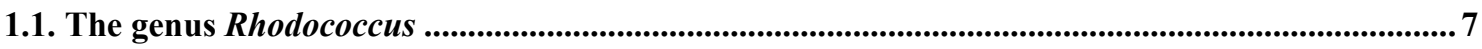

1.2. Environmental and industrial applications of Rhodococcus ...................................................9

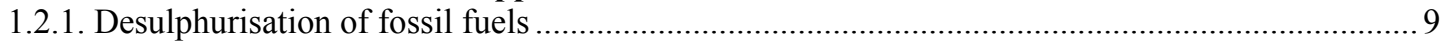

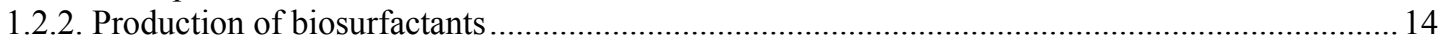

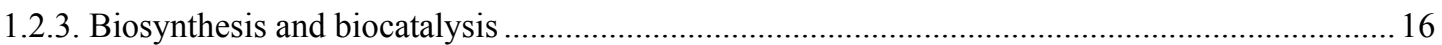

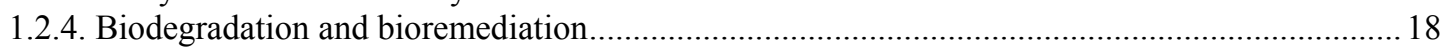

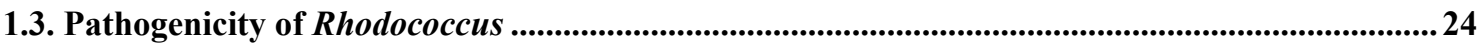

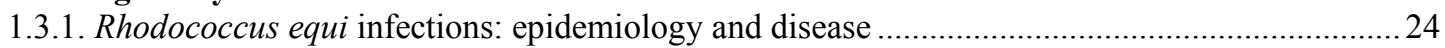

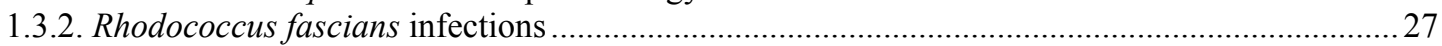

1.4. Mechanisms of starvation survival in bacteria .............................................................................28

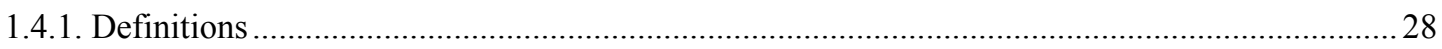

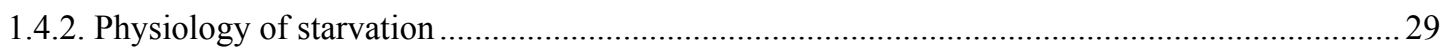

1.4.3. Molecular components of regulation of the Starvation Survival Response (SSR) in Gram-

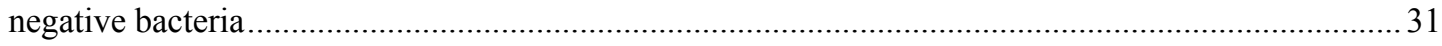

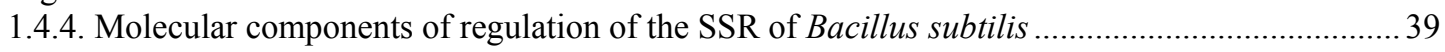

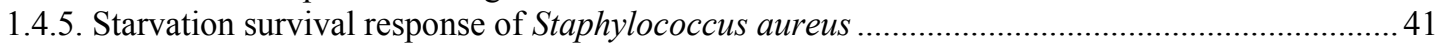

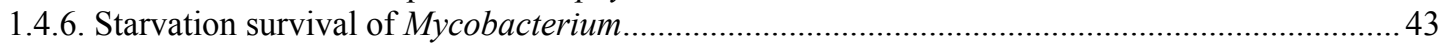

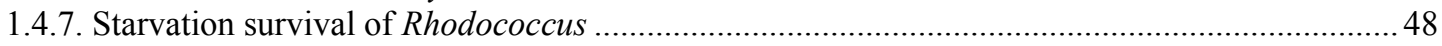

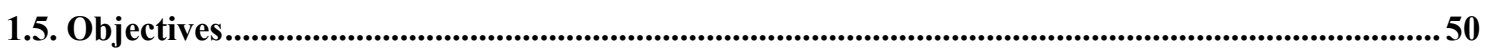

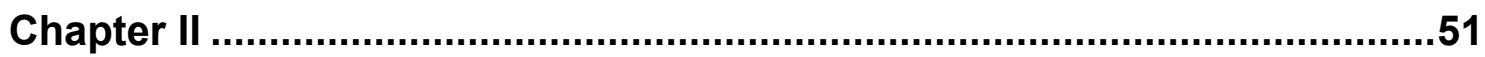

Materials and Methods ....................................................................

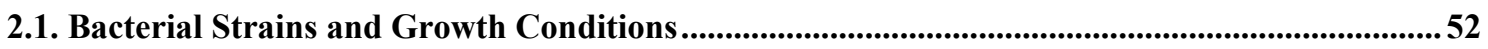

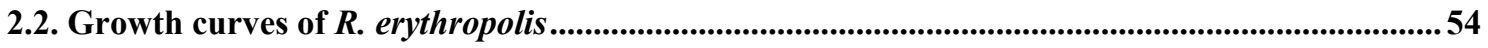

2.3. Measurement of impact of heat shock on cell viability .........................................................57

2.4. Measurement of impact of oxidative damage on cell viability.....................................................58

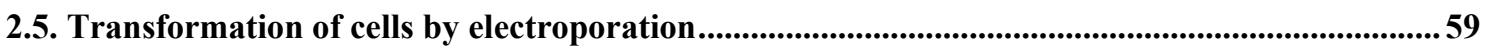

2.5.1. Preparation of electrocompetent cells of $E$. coli and Rhodococcus ..........................................59

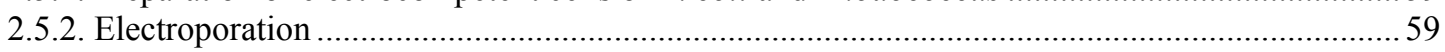

2.6. Transposon mutagenesis and generation of mutant bank ...........................................................61

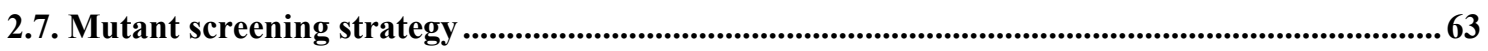

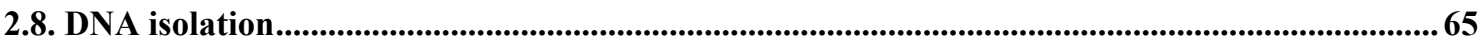

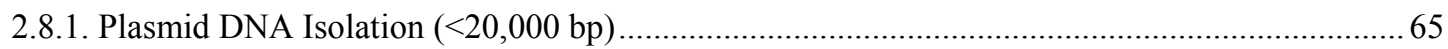

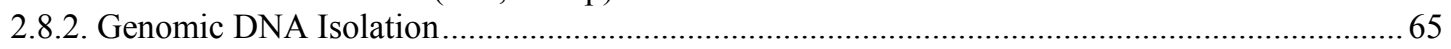

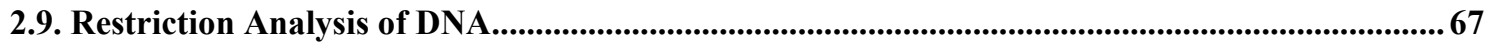




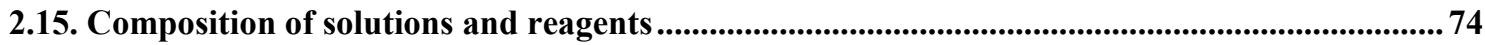

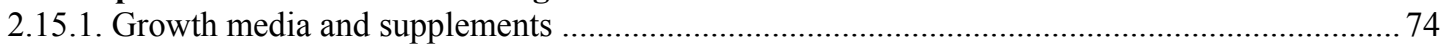

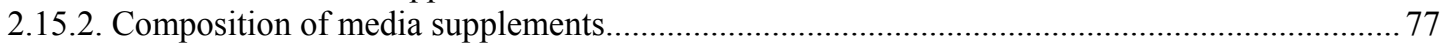

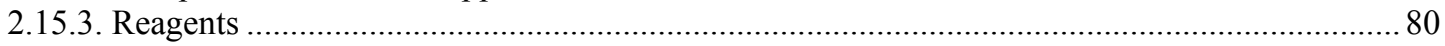

Chapter III .81

Physiological Response of Rhodococcus erythropolis SQ1 to Starvation

3.1. Selection of an appropriate Rhodococcus strain for the study of carbon starvation ....................83

3.1.1. Design of a chemically defined medium suitable for studying the effect of carbon starvation on

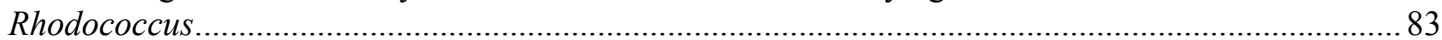

3.1.2. Antibiotic resistance markers for screening of transformants and mutants of Rhodococcus.......87

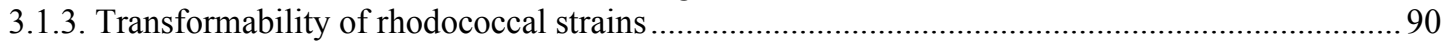

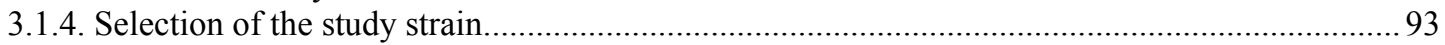

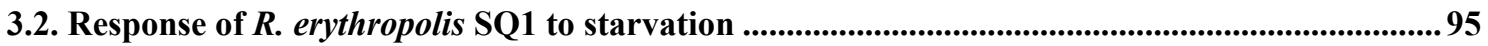

3.2.1. Response of $R$. erythropolis SQ1 to growth in complex medium and to multiple-nutrient

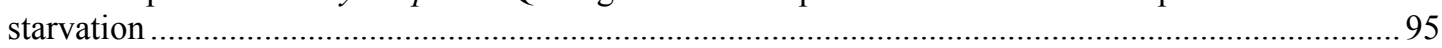

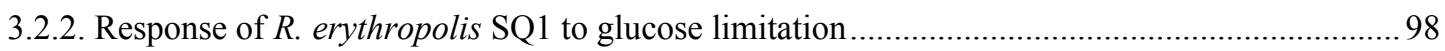

3.3. Starvation of $R$. erythropolis SQ1: Cross protection induced by carbon starvation................... 109 3.3.1. The effect of carbon-starvation on the ability of $R$. erythropolis SQ1 to survive heat-shock.... 110

3.3.2. Cross-protection against oxidative stress..................................................................... 117

Chapter IV ....................................................................................

Random Transposon Mutagenesis of $R$. erythropolis SQ1 and Genomic Analysis of the Mutants.........................................................................122

4.1. Generation of a mutant bank of $R$. erythropolis SQ1 ............................................................125

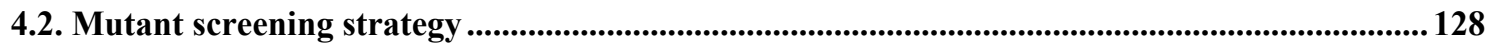

4.3. Growth curves of selected mutants in chemically defined medium ........................................ 138

4.4. Identification of genes and functions disrupted by transposon insertion ................................... 142

4.4.1. Nucleotide excision repair mutant 4G6: $u v r B$ mutant ................................................ 148

4.4.2. Mutant with insertion downstream of putative IMP dehydrogenase gene guaB: mutant 10D3 155

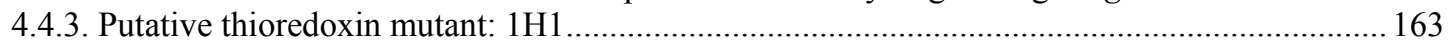

4.4.4. Histidine and Serine/Glycine auxotrophic mutants ........................................................ 168

4.4.5. Serine/glycine auxotrophic ser $A$ mutant: 6E6 ............................................................ 185

Chapter V ...........................................................................................193

Discussion ........................................................................................193

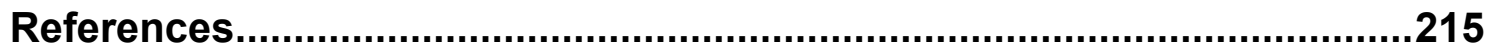




\section{Figure list}

\section{Chapter I}

Figure 1.1 Desulphurisation of DBT into HBP by R. erythropolis IGTS8.

Figure 1.2 Transcriptional and translational regulation of rpoS, leading to

synthesis of $\sigma^{\mathrm{S}}$.

Figure 1.3 Recognition and degradation pathway of $\sigma^{S}$

\section{Chapter II}

Figure 2.1 Schematic overview of the growth experiments methodology..... 56

Figure 2.2 Generation of a mutant bank by random transposon insertion using the EZ-Tn5 Transposome (@2007 Epicentre Biotechnologies).

Figure 2.3 Mutant screening strategy (adapted from Uhde et al., 1997)..........64

Figure 2.4 Principle of Inverse PCR (iPCR) and cloning...............................70

\section{Chapter III}

Figure 3.1 Growth curve of $R$. erythropolis SQ1 grown in LB and distilled water.

Figure 3.2 Growth curve of $R$. erythropolis SQ1 grown in CDM IA $1 \%$ glucose and $0.1 \%$ glucose with static incubation after entry into stationary phase........99 Figure 3.3 Comparison of the growth rates of $R$. erythropolis SQ1 grown in CDM IA $1 \%$ ( $\mathbf{a})$ and $0.1 \%$ glucose ( $\square$ ). 100

Figure 3.4 Growth curve of $R$. erythropolis SQ1 grown in CDM IIA $1 \%$ glucose and CDM IIA $0.1 \%$ glucose with static incubation.

Figure 3.5 Growth curve of $R$. erythropolis SQ1 grown in CDM II $1 \%$ glucose

and CDM II $0.1 \%$ glucose with or without static incubation 105

Figure 3.6 Representative standard curves for measurement of glucose in CDM IIA.

Figure 3.7 Monitoring of glucose concentration during growth of $R$. erythropolis

SQ1 in (a) CDM IIA 1\% glucose and (b) CDM IIA 0.1\% glucose...................108 Figure 3.8 Preliminary heat shock experiment in which $R$. erythropolis SQ1 cultures were exposed to heat treatment at $57{ }^{\circ} \mathrm{C}$

Figure 3.9 Effect of heat shock on diluted and undiluted late exponential phase cultures of $R$. erythropolis SQ1

Figure 3.10 Survival of $R$. erythropolis SQ1 subjected to heat shock $\left(57^{\circ} \mathrm{C}\right)$ with and without exposure to $40 \mathrm{\mu g} \mathrm{ml}^{-1}$ chloramphenicol...............................116 Figure 3.11 Effect of increasing concentrations of tBHP on exponentially growing $R$. erythropolis SQ1 cells.

Figure 3.12 Survival of $R$. erythropolis SQ1 subjected to oxidative stress (400 mM tBHP) with and without exposure to $40 \mu \mathrm{g} \mathrm{ml}^{-1}$ chloramphenicol.....121

\section{Chapter IV}

Figure 4.1 Agarose gel (0.8\%) electrophoresis of the PCR products using KANR-FP and KANR-RP, designed to the Tn903 kanamycin resistance

gene

Figure 4.2 Appearance of mutants growing on CDM IIA $1 \%$ glucose agar.....130

Figure 4.3 Growth curves of mutants 4G6 (a) and 10D3 (b) compared to wild-

type $R$. erythropolis SQ1. 
Figure 4.4 Growth curves of mutants 1B2 (a) and 1H1 (b) compared to wild-type R. erythropolis SQ1................................................................... 141

Figure 4.5 Principle of recovering DNA flanking a transposon insertion site by inverse PCR (iPCR) ..........................................................................143

Figure 4.6 Total genomic DNA from mutant $1 \mathrm{H} 1$ subjected to restriction endonucleases and analysed by agarose gel electrophoresis.

Figure 4.7 Agarose gel electrophoresis of DNA products of inverse PCR on religated DNA from mutant $1 \mathrm{H} 1$

Figure 4.8 Schematic representation of the $1671 \mathrm{bp}$ sequence retrieved for mutant 4G6.

Figure 4.9 Alignment of the putative UvrB sequence from R. erythropolis SQ1 with sequences from Rhodococcus sp. RHA1, Nocardia farcinica IFM 10152, M. tuberculosis H37Rv and M. vanbaalenii PYR-1..................................152 Figure 4.10 Schematic representation of the $1165 \mathrm{bp}$ sequence retrieved for mutant 10D3.

Figure 4.11 Alignment of a putative IMP dehydrogenase sequence from $R$. erythropolis SQ1 with sequences from Rhodococcus sp. RHA1 (guaB1), Nocardia farcinica IFM 10152 (guaB), and Mycobacterium leprae TN (guaB2).....

Figure 4.12 Position of the inositol-5'-monophosphate dehydrogenase in the purine metabolism pathway [adapted from the Rhodococcus sp. RHA1 pathway from KEGG (Kanehisa et al., 2006)]....

Figure 4.13 Schematic representation of the gua operon of E. coli K12, $N$. farcinica IFM 10152, M. tuberculosis H37Rv and Rhodococcus RHA1..........162 Figure 4.14 Alignment of a putative thioredoxin from $R$. erythropolis SQ1 with sequences from Rhodococcus sp. RHA1, Mycobacterium gilvum and Nocardia farcinica IFM 10152.

Figure 4.15 Schematic representation of the 844 bp sequence retrieved for mutant $1 \mathrm{H} 1$

Figure 4.16 Alignment of the sequences obtained from mutants 1B3, 3B10,

$4 \mathrm{G} 11$ and 10E4

Figure 4.17 Schematic representation of the genomic area (1317 bp) disrupted in mutants $1 \mathrm{~B} 3,3 \mathrm{~B} 10,4 \mathrm{G} 11$ and $10 \mathrm{E} 4$.

Figure 4.18 Alignment of a putative HisA sequence from $R$. erythropolis SQ1 with HisA sequences from Rhodococcus sp. RHA1 and Nocardia farcinica IFM 10152 (Noc), and with HisA/TrpF sequences from Mycobacterium vanbaalenii PYR-1 (PYR) and Mycobacterium sp. MCS

Figure 4.19 Function of HisA in the synthesis of histidine............................175

Figure 4.20 Schematic representation of the $1232 \mathrm{bp}$ sequence retrieved from mutant 3B4.

Figure 4.21 Alignment of a putative HisB sequence from R. erythropolis SQ1 with HisB sequences from Rhodococcus sp. RHA1, Nocardia farcinica IFM 10152 (Noc), Mycobacterium avium subsp. paratuberculosis K-10 and M. tuberculosis H37Rv.

Figure 4.22 Steps catalysed by HisB (highlighted in red) in the histidine anabolic pathway [adapted from KEGG (Kanehisa et al., 2006)] 182 Figure 4.23 Histidine synthesis operons of E. coli K-12, M. tuberculosis H37Rv and Rhodococcus sp. RHA1...

Figure 4.24 Schematic representation of the $831 \mathrm{bp}$ sequence retrieved for mutant 6E6. 
Figure 4.25 Alignment of a putative SerA sequence from $R$. erythropolis SQ1 with sequences from Rhodococcus sp. RHA1, M. tuberculosis strain H37Rv, M. vanbaalenii PYR-1 and N. farcinica IFM 10152.................................188 Figure 4.26 Position of phosphoglycerate dehydrogenase in a simplified glycine, serine and threonine metabolism pathway [adapted from the Rhodococcus $\mathrm{sp}$. RHA1 pathway from KEGG (Kanehisa et al., 2006)] 189

\section{Chapter V}

Figure 5.1 Transposon insertion sites identified in mutants 1B3, 3B10, 4G6, 10E4, 3B4, 10D3, 6E6 and 4G6. 
Chapter I

\section{Introduction}




\subsection{The genus Rhodococcus}

Members of the genus Rhodococcus are described in Bergey's manual as aerobic, Gram positive, non-motile, mycolate-containing, partially acid-fast, nocardioform actinomycetes. The shape of cells during growth ranges from cocci to filaments with short projections and elementary branching (Goodfellow, 1989). According to 16S phylogenetic clustering, they are part of the Corynebacterineae, which includes Grampositive actinomycetes with mycolic acid in the cell wall (Stackebrandt et al., 1997).

Over the last seven decades there has been confusion surrounding the taxonomy of the mycolic acid-containing actinomycetes, which currently includes Rhodococcus, Corynebacterium, Dietzia, Gordonia, Mycobacterium, Nocardia, Skermania, Millisia, Segniliparus, Williamsia, Tsukamurella and Smaragdicoccus (Adachi et al., 2007; Butler et al., 2005; Goodfellow et al., 1998; Kampfer et al., 1999; Soddell et al., 2006; Stackebrandt et al., 1997). This is largely due to the inability of conventional tests to distinguish amongst different species. In the last ten years, methods have been developed to distinguish Rhodococcus from other members of the mycolata group. A study by Bizet et al. (1997) showed that pure cultures of Rhocococcus, Gordonia and Dietzia could be discriminated using a carbon source utilisation test based on the "Biotype-100" strips (Biomérieux), in which growth on 99 different carbon sources is analysed with the computer package Taxotron (Taxolab). The system could differentiate between 29 different species, including 10 Rhocococcus, 7 Gordonia and 1 species of Dietzia (Bizet et al., 1997). Primers and specific probes based on the 16S rRNA sequences have also been designed for identification of $R$. equi (Bell et al., 1996), R. globerulus, R. erythropolis, R. opacus and R. ruber (Bell et al., 1999), and R. coprophilus (Savill et al., 2001). In addition, primers specific to the vap virulence 
genes of the $R$. equi virulence plasmid have been designed to differentiate between virulent and avirulent equine-derived strains (Takai et al., 1998).

The name "Rhodococcus" was first used by Overbeck in 1891, and was subsequently reintroduced by Tsukamura in 1974 (Overbeck, 1891; Tsukamura, 1974). Study of the lipid composition of coryneform bacteria led to further determination of what was called the "rhodochrous" complex (Minnikin et al., 1977). Following a study of 177 strains examined for 92 unit characters (such as colony morphology, growth on sole carbon source, growth temperature), the taxon Rhodococcus was definitely established in 1977 (Goodfellow \& Alderson, 1977).

The genus is defined primarily on the basis of cell-wall composition. It is restricted to actinomycetes that have: (a) A1 $\gamma$ peptidoglycan consisting of $\mathrm{N}$-acetylglucosamine, $\mathrm{N}$ glycolylmuramic acid, D- and L-alanine, and D-glutamic acid with meso-DAP as the diamino acid; (b) arabinose and galactose as diagnostic wall sugars (chemotype IV and whole-cell sugar pattern type A); (c) a phospholipid pattern consisting of diphosphatidylglycerol, phosphatidylethanolamine, phosphatidylinositol, and phosphatidylinositol mannosides; (d) a fatty acid profile containing major amounts of straight-chain, unsaturated, and tuberculostearic acids (type IV), and mycolic acids with 32-54 carbon atoms; and (e) dehydrogenated menaquinones with eight or nine isoprene units (Briglia et al., 1996; Goodfellow, 1989; Li et al., 2004). The mol\% G+C of the DNA ranges from 55.5 to 72 mol\% (Goodfellow et al., 1998; Li et al., 2004).

16S rRNA studies subsequently indicated that Rhodococcus was indeed a genus of its own and, even though there have been minor changes over the last decade or so, most of the species described as Rhodococcus are now well characterised (Goodfellow et al., 
1998). More recent work on 29 R. erythropolis strains showed very little diversity in the 16S rRNA sequence (Oberreuter et al., 2002). There are now forty-one officially recognised species of Rhodococcus (Euzéby, 2007).

\subsection{Environmental and industrial applications of Rhodococcus}

Rhodococci have been isolated from a large range of habitats such as soil, rocks, ground water, marine sediments, animal dung, the guts of insects and from infected animals, plants and humans (Goodfellow, 1989; Rowbotham \& Cross, 1977; Tilford, 1936; Weyland, 1969; Woolcock et al., 1979). They have been isolated from environments ranging from the deep sea (Heald et al., 2001) to the Arctic and Antarctic soil (Bej et al., 2000; Kochkina et al., 2001), and there are even reports of isolation from the air in the Mir space station (Li et al., 2004). Members of the genus Rhodococcus have an outstanding range of enzymes that enable them to transform many chemicals, rendering them extremely attractive for many industrial processes, as well as good candidates in environmental biotechnology (Warhurst \& Fewson, 1994). This is reflected in the number of patents exploiting Rhodococcus; a search for the word "Rhodococcus" at http://openaccess.dialog.com/ip/ in all patent sources yielded over 1000 titles in October 2007. This section of the review will focus on the environmental and industrial applications of the genus Rhodococcus, with an added emphasis on the species R. erythropolis.

\subsubsection{Desulphurisation of fossil fuels}

The combustion of fossil fuels releases sulphur oxides in the atmosphere, contributing to acid rain and air pollution. To control emissions, environmental agencies worldwide impose strict regulations on the sulphur content of fuels. The United States and European Union environment protection agencies have imposed regulations (Directive 
98/70/EC of the European parliament) that allow a maximum sulphur content of $15 \mathrm{ppm}$ since 2006 (Anonymous, 1998). A new objective will be to bring the concentration of sulphur in fuels to $10 \mathrm{ppm}$ by 2009 , if new legislation currently proposed by the European Commission is adopted (Anonymous, 2007).

The conventional technique to remove sulphur from petroleum is through a process called hydrodesulphurisation (HDS), which involves reacting oil fractions at high temperature and pressure with an inorganic catalyst and hydrogen, resulting in the production of hydrogen sulphide and a desulphurised product (Gray et al., 1996). This technique is very energy-demanding, and some compounds are recalcitrant to it, for example benzothiophene, dibenzothiophenes (DBT) and DBT with substitutions adjacent to the sulphur group (McFarland, 1999). Several organisms capable of desulfurising DBT have been isolated, in particular Rhodococcus erythropolis. R. erythropolis IGTS8 (ATCC 53968), patented by the Institute of Gas Technology (Des Plaines, IL, USA), can oxidise DBT to 2-hydroxybiphenyl (HBP), releasing inorganic sulphur that can then be assimilated by the bacteria (Kilbane \& Jackowski, 1992). Other strains that can desulphurise fuel have been isolated, such as $R$. erythropolis KA2-5-1 (Hirasawa et al., 2001), R. erythropolis H-2 (Ohshiro et al., 1995) and $R$. erythropolis D-1 (Ohshiro \& Izumi, 2000). Importantly, the phenyl rings of DBT are left intact following desulphurisation by R. erythropolis, and the HBP (2hydroxybiphenyl) is returned to the fuel, hence its calorific (i.e. fuel) value is not diminished (Kayser et al., 1993).

The oxidation pathway of DBT has been characterised, and the enzymes DszA, DszB, DszC and DszD identified (Denome et al., 1993; Denome et al., 1994). DszA and DszC are flavin-dependent monooxygenases, particular in that they use $\mathrm{FMNH}_{2}$ as a co- 
substrate instead of FAD. DszB is the desulfinase that removes the sulphur, and DszD is a FMN:NADPH reductase that supplies DszA and DszC with $\mathrm{FMNH}_{2}($ Oldfield et al., 1997) (see Figure 1.1).

A number of steps in the pathway are still not well understood, including for example the transfer of these hydrophobic polyaromatic molecules into the cell (Monticello, 2000). The genes involved in the basic desulphurisation steps have been characterised. $d s z A, d s z B$ and $d s z C$ have been located in a single operon on a $150 \mathrm{~kb}$ circular plasmid in $R$. erythropolis IGTS8, and on a $100 \mathrm{~kb}$ linear plasmid in related strains (DenisLarose et al., 1997; Oldfield et al., 1997). Their transcription is repressed by the presence of sulphur-containing amino acids, but not by DBT (Li et al., 1996). 

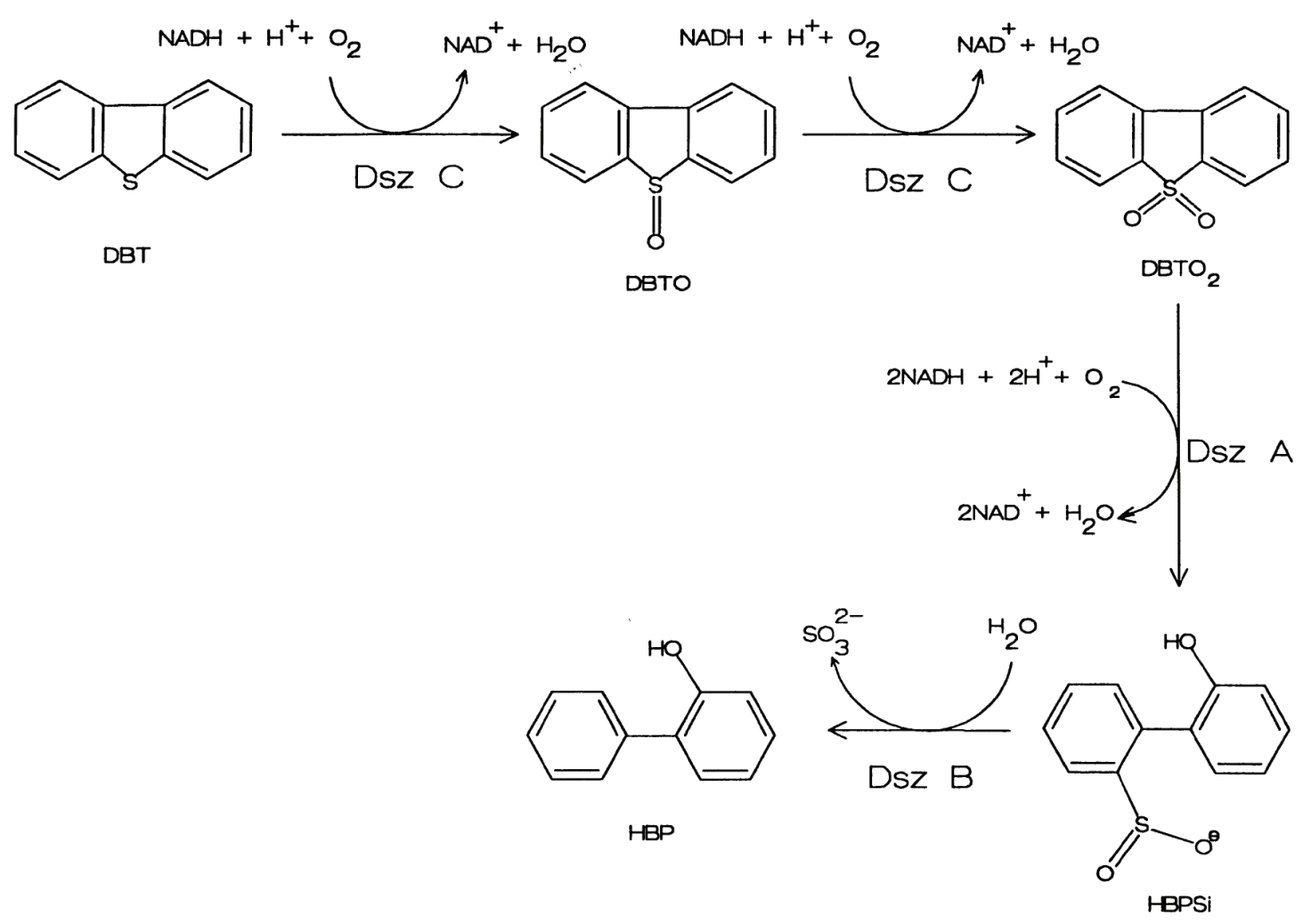

Figure 1.1. Desulphurisation of DBT into HBP by $R$. erythropolis IGTS8. Reproduced from Oldfield et al. (1997). DBT, dibenzothiophene; DBTO, dibenzothiophene 5-oxide; $\mathrm{DBTO}_{2}$, dibenzothiophene 5,5-dioxide; HBPSI, 2-(2'Hhydroxyphenyl)benzene sulphinate; HBP, 2-hydroxybiphenyl. 
Exploiting the biochemical and genetic information available on the desulphurisation pathway of Rhodococcus and indeed other organisms, it was possible to consider manipulating these organisms to produce improved biocatalysts. Work has been done to improve the desulphurisation rate, for example by insertion of multiple copies of the $d s z$ operon in R. erythropolis I19 (Folsom et al., 1999). An attempt has also been made to increase the desulfinase enzyme activity and broaden its specificity by error-prone PCR of the $d s z B$ gene (Borole et al., 2003). A clone with higher desulfinase activity was isolated, but analysis of the $d s z B$ sequence showed the mutation was not within the gene and therefore it is assumed that the modification took place in a regulatory sequence.

There are still many hurdles to overcome before biodesulphurisation of fuels becomes a routine process, including the stability of the biocatalyst. BDS is performed using resting cells, meaning they are non-growing live cells (Guerinik \& Al-Mutawah, 2003; Noda et al., 2002; Oldfield et al., 1997). An advantage of using resting cells is that they can be used at higher concentrations than growing cells (Abbad-Andaloussi et al., 2003). Resting cells, harvested at the end of exponential phase for maximum activity, are usually washed and resuspended in potassium phosphate or HEPPS [4-(2hydroxyethyl)-1-piperazine-propane-sulfonic acid] buffer to which the fuel is added and mixed. Due to the hydrophobicity of $R$. erythropolis, the cell suspension can form a very fine and stable emulsion with the oil, which increases the conversion rate of DBT to HBP (Borole et al., 2002). This, however, does make it difficult to separate the biocatalyst from the reaction medium after the fuel was desulphurised. As a result, an immobilised $R$. erythropolis KA-2-5-1 resting cell system has been developed (Naito et al., 2001). This biocatalyst was found to function well and, when reactivated by incubation overnight in growth medium, could maintain its activity for up to 900 hours. 


\subsubsection{Production of biosurfactants}

Biosurfactants are produced by micro-organisms and represent a broad spectrum of molecules, including normal and hydroxyl fatty acids, glycolipids, cyclic lipopeptides, $N$-acyl amino acids, lipopeptides, mono and diglycerides, and phospholipids. Due to their amphiphatic nature, they have in common their capacity to accumulate at airwater, oil-water and solid-liquid interfaces (for a review, see Christofi \& Ivshina, 2002; Lang, 2002). As a result they have solubilisation, emulsification, dispersion, wetting, foaming and detergent effects. The diversity of chemical structures and surface properties may reflect differences in their role in the microorganisms' natural habitat. For example, several biosurfactants increase the bioavailability of hydrophobic substrates (including many xenobiotics), while others have antimicrobial activities (for a review, see Ron \& Rosenberg, 2001). Synthetic surfactants have been used in oil washing for secondary oil recovery and to clean oil pipes and oil reservoirs. The impact of biosurfactants on the biodegradation of xenobiotic contaminants in soil and other environments has been investigated and showed that biosurfactants can be used to speed up the remediation of organic and metal-contaminated sites, and their low toxicity makes them suitable for bioremediation by bioaugmentation (for a review, see Christofi \& Ivshina, 2002). They are believed to help cell adhesion to hydrophobic phases in twophase systems, help the entry of hydrophobic compounds into the cell by decreasing the interfacial tension and disperse hydrophobic compounds to increase the surface area for bacteria-molecule contact (Finnerty, 1994). This is illustrated by the increased desulphurisation of fuel by $R$. erythropolis in the presence of surfactants (Feng et al., 2006; Patel et al., 1997). A similar observation was made with $R$. erythropolis EK-1 and surfactants produced by a Pseudomonas strain (Karpenko et al., 2006). Similarly, mycolic acids enhanced the degradation of diesel by Rhodococcus baikonurensis (Lee et al., 2006). 
The predominant biosurfactants produced by members of the genus Rhodococcus are glycolipids, with trehalose as the hydrophilic compound and mycolates as the hydrophobic component (Lang \& Philp, 1998). The type and quantity of biosurfactant produced by Rhodococcus is dependent on growth conditions including growth phase, nutrient limitation and exposure to hydrophobic substrates such as $n$-alkanes. Nongrowth-associated production of trehalose lipids has also been observed (Kim et al., 1990). R. erythropolis can produce biosurfactants such as trehalose mono- and dicorynomycolates, trehalose-6,6'-diacylates and trehalose-6-acylates and trehalose tetraester, as well as common glycolipids and lipids such as cetyl alcohol, palmitic acid, methyl $n$-pentadecanoate, triglyceride and mycolic acids (Lang \& Philp, 1998; Pirog et al., 2004). It was shown that high yields of up to $20 \mathrm{~g} / 1$ glycolipids could be obtained with resting cells of $R$. erythropolis DSM 43215 (Kim et al., 1990). Production of efficient surfactants (unidentified, monitored by measuring medium surface tension) was also observed in R. erythropolis strains DSM 1069 and TA57 (Pizzul et al., 2006). R. ruber can also produce a trehalose dicorynomycolate in large quantities (approx. $10 \mathrm{~g} / \mathrm{l})$ under nitrogen-limited conditions, as described by Philp et al. (2002), that is more hydrophobic than that produced by $R$. erythropolis. 


\subsubsection{Biosynthesis and biocatalysis}

There are many advantages to the use of biocatalysts in chemical synthesis. In particular, complex reactions can be performed in a single step, leading to the use of fewer and cheaper reagents. High yields and high product purity can also be achieved. Milder reaction conditions, typically at near ambient temperature and pressure, can also reduce the cost of the operation (Johannes, 1996; Thomas et al., 2002). Biocatalysis has also been proposed as a way to shift the current main source of chemicals from oil to biomass, thereby reducing $\mathrm{CO}_{2}$ emissions and hopefully leading to "greener" chemistry (Danner \& Braun, 1999). Rhodococcus species have been found to catalyse reactions that have applications in the synthesis of fine chemicals (e.g. perfumes, paints), pharmaceuticals and sensors, and more, a selection of examples are presented below.

The most successful use of Rhodococcus species in an industrial setting so far has been the production of acrylamide by Rhodococcus rhodochrous J1 (Nagasawa \& Yamada, 1990). Each year more than 30,000 tons of acrylamide are produced, with a productivity of over $7 \mathrm{~kg}$ acrylamide per g of cells (Nagasawa et al., 1993), using non-growing cells fixed inside acrylamide beads [see US patent 7,205,133 by Banba et al. (2002)].

Resting cells of R. erythropolis DSM 1069 were shown to synthesise cyclopropanol from cyclopropyl methyl ketone (Overbeeke et al., 2003). This reaction has potential for industrial exploitation, as cyclopropanol is difficult to produce by traditional organic chemistry methods and is thus not commercially viable. The possibility to produce cyclopropanol in large quantities for a reasonable cost would open new ways in organic synthesis that could not be investigated before. Another example of potential industrial application came from the observation that $R$. erythropolis DCL14 could grow on limonene as a source of carbon (van der Werf et al., 1999b). This led to the discovery of 
enzymes that produce enantiopure epoxides (van der Werf et al., 1999a), essential building blocks in the synthesis of fine chemicals used as fragrances and flavours. R. erythropolis IGTS8 and R. erythropolis IGTS8 BKO-53, a R. erythropolis IGTS8 derivative lacking $d s z A$ and $d s z B$ but containing multiple copies of $d s z C$, originally developed for biodesulphurisation of fuels, were used to transform simple sulphides into chiral sulfoxides, thus providing interesting building blocks for organic chemistry (Holland et al., 2003a; Holland et al., 2003b). The bioconversion was mediated by resting cells, resuspended in a phosphate buffer containing glucose, ethanol and the appropriate sulphide during a period of 15 hours. In addition to substituted benzyl sulphides, the organism mediated sulphide oxidation of amino acids such as methionine and cysteine.

R. ruber strain DSM 44541 was found to transform sec-alcohols into ketones and vice versa by an asymmetric hydrogen transfer that uses 2-propanol or acetone as a cofactor (Kosjek et al., 2003). Optically active $R$ or $S$ alcohols, which are important as intermediates for the synthesis of fine chemicals, are produced depending on the substrate. $R$. ruber has been shown to produce dodecanedioic acid, which is an intermediate in the synthesis of films, fibres, paints and adhesives (Thomas et al., 2002) and to synthesise poly(3-hydroxyalkanoic acid) (PHA), a polymer that can be used to produce biodegradable plastics (Madison \& Huisman, 1999).

The exploitation of resting cells of R. erythropolis SC 13845 and R. erythropolis 16002 as biocatalysts in the production of the HIV-protease inhibitor Atazanavir is being considered. A key step in the process of Atazanavir production includes a diastereoselective reduction which can be mediated by specific $R$. erythropolis isolates with a diastereomeric purity of $>98 \%$ and minimum production of undesirable end- 
products (Patel et al., 2003). Rhodococcus sp. strains B264-1 and I24 were found to convert indene to indandiol, an essential step in the synthesis of another HIV-protease inhibitor, CRIXIVAN (Buckland et al., 1999).

The ability to survive adverse environmental conditions, including high concentrations of toxicants (de Carvalho et al., 2004; Paje et al., 1997), combined with their ability to metabolise a wide range of diverse and unusual compounds (e.g. substituted phenols), offers a rich resource for the development of biosensors capable of detecting these toxic compounds in the environment (de Carvalho \& da Fonseca, 2004). Potential sensors currently being developed or evaluated include the use of whole cells of $R$. erythropolis for the detection of 2,4-dinitrophenol (Emelyanova \& Reshetilov, 2002). R. ruber and $R$. erythropolis also have the capacity to degrade the industrial pollutant acrylonitrile, prompting the development of a biosensor relying on the transformation of acrylonitrile into ammonium acrylate by $R$. ruber (Roach et al., 2003). Another field of interest is the oxidation of cholesterol. $R$. erythropolis can oxidise cholesterol, which leads to various applications (Jadoun \& Bar, 1993). Work is being carried out towards the isolation and purification of the cholesterol oxidase of $R$. erythropolis, with the aim of producing a test or a sensor to detect cholesterol (Sojo et al., 1997; Sojo et al., 2002). Disruption of the cholesterol metabolism pathway could also produce mutants that accumulate bioactive steroids, or potential precursors in the synthesis of bioactive steroids (van der Geize et al., 2001).

\subsubsection{Biodegradation and bioremediation}

Rhodococcus species can metabolise a wide range of xenobiotics, from simple hydrocarbons through to chlorinated and aromatic hydrocarbons, nitroaromatics and chlorinated polycyclic aromatics such as polychlorinated biphenyls (PCBs) (Warhurst \& 
Fewson, 1994). This makes them potentially useful in bioremediation. A few specific examples are described below.

A wide range of chemicals, in particular aromatics and hydrocarbons, can be degraded by Rhodococcus. Natural isolates have been found to degrade polychlorinated biphenyls (PCBs), a common pollutant from industrial oils (Begonja Kolar et al., 2007). R. erythropolis UPV-1 can degrade phenol and formaldehyde, and processes are being investigated to use it for decontamination of industrial wastewater (Begoña Prieto et al., 2002; Hidalgo et al., 2002; Prieto et al., 2002). R. erythropolis M1 can degrade 2chlorophenol, phenol and $\rho$-cresol in co-culture with Pseudomonas fluorescens (Goswami et al., 2005). R. erythropolis HL PM-1 can degrade dinitrophenol (Kitova et al., 2004), and R. erythropolis DSM 1069 and TA57 have been found to metabolise polyaromatic hydrocarbons (Pizzul et al., 2006). Additionally, R. erythropolis VKM AS-1339D degrades oil and oil products (Yagafarova et al., 2002). Screening of contaminated soil from a chemical plant for benzene degraders resulted in the isolation of a Rhodococcus strain of particular interest due to its ability to use liquid benzene as a sole carbon source and at concentrations observed to be toxic to other bacteria. This strain can also co-metabolise toluene and use chlorinated benzenes, making it an ideal candidate for bioremediation (Paje et al., 1997). The persistence of Rhodococcus is illustrated by their isolation from soil in Antarctica (Bej et al., 2000). These species were shown to degrade a wide range of alkanes (C6-C20 and branched alkane pristane), as well as being able to grow at temperatures ranging from $-2{ }^{\circ} \mathrm{C}$ to $+15^{\circ} \mathrm{C}$. These characteristics would make these species useful in bioremediation of cold environments where oil is extracted, such as Alaska or Siberia. 
$R$. ruber also can degrade a large number of pollutants. An example is ethyl tert-butyl ether, a common pollutant from petrol (Chauvaux et al., 2001). Like R. erythropolis, some strains of $R$. ruber can degrade PCBs (Begonja Kolar et al., 2007). Most interestingly, $R$. ruber can metabolise polyethylene, one of the most widespread and most recalcitrant plastic pollutants known (Gilan et al., 2004), which could lead to the bioremediation of landfill.

Strains of $R$. erythropolis have been found to degrade fertilisers (isobutylidenediurea, Jahns \& Schepp, 2001), carcinogenic toxins (aflatoxin B1, Alberts et al., 2006), C5C16 hydrocarbons and motor oils (de Carvalho \& da Fonseca, 2005b), diesel (Lin et al., 2005) and to dehalogenate haloalkanes (Armfield et al., 1995). For a review of the metabolic capacities of $R$. erythropolis, see de Carvalho \& da Fonseca (2005a).

Rhodococcus strain DN22 has been found to readily degrade RDX (hexahydro-1, 3, 5trinitro-1, 3, 5-triazine) in vitro. RDX is a commonly used explosive, hence a rather frequent pollutant. Since these compounds are toxic and mutagenic, it is important to be able to clean up sites where vast quantities of explosives have been used or stocked, for example by a bioremediation process. Several bacteria are known to biodegrade RDX either aerobically or anaerobically, including Rhodococcus strain DN22 (Coleman et al., 1998) and Enterobacteriaceae (Kitts et al., 1994), respectively. Initial studies of the RDX biodegradation pathway in Rhodococcus strain DN22 indicate that the system is inducible and that the initial steps may be mediated by a cytochrome p-450 system. Coleman et al. (2002) suggest that the cytochrome p-450 enzyme is plasmid-encoded. This is of significance since this would enable transfer to other bacteria. 
Although in vitro studies are important in order to identify the specific organisms involved in biodegradation of a particular toxicant and the metabolic pathways involved, investigation of the performance of individual isolates or microbial consortia in contaminated soil and/or water is absolutely essential. Factors such as the competing indigenous microflora and environmental conditions impact on the survival and catabolic activity of the introduced organism. A number of studies have been initiated, investigating the biodegrading activity of Rhodococcus sp. in soil.

The activity of the naphthalene-degrading Rhodococcus strain 1BN was investigated in naphthalene-contaminated soil in the presence and absence of an indigenous population of naphthalene-degrading bacteria (Cavalca et al., 2002). Rhodococcus strain 1BN was able to survive in the naphthalene-contaminated soil in the presence of the competing indigenous naphthalene-degrading microflora, constituting $13.6 \%$ of the naphthalenedegrading population on the $22^{\text {nd }}$ day of the experiment. Although naphthalene degradation was not significantly different in soil supplemented with Rhodococcus strain $1 \mathrm{BN}$ compared to uninoculated soil, the presence of $1 \mathrm{BN}$ resulted in faster mineralisation of the parent compound to $\mathrm{CO}_{2}$. Screening of the soil microflora by PCR amplification of narA (Gram-positive) and ndoB/nahAC (Gram-negative) naphthalenedegrading dioxygenase genes revealed that, although after 10 days the $n d o B$-harbouring strains (Gram-negative) were dominant, at 22 days the dominant isolates contained narA (Gram-positive). This would suggest that, in substrate-limiting conditions, Rhodococcus and other Gram-positive bacteria survive longer than Gram-negative bacteria. Due to their broad metabolic activity, Gram-positive bacteria such as Rhodococcus also bring additional degradative properties to the microcosm, for example towards medium- and long-chain alkanes. Similar observations on population changes were made during a study of crude oil biodegradation in soil under laboratory 
conditions. Gram-negative bacteria were predominant during the first seven months after which Gram-positive bacteria, including indigenous Rhodococcus species, were dominant (Peressutti et al., 2003).

The range of chemicals degraded and metabolised by Rhodococcus species is impressive, as outlined above and in several reviews (de Carvalho \& da Fonseca, 2005a; Warhurst \& Fewson, 1994). With the development of genetic tools for Rhodococcus (see e.g. Veselý et al., 2003) and the recent availability of complete genome sequences (McLeod et al., 2006), there is a great scope for the production of genetically engineered "one-cell factories" to use in industry, and for the production of strains that can help clean up the environment from toxicants. What is striking with most of these industrial processes is that they use resting cells (see e.g. Abbad-Andaloussi et al., 2003; Caro et al., 2007; Kim et al., 1990). The cells are grown in the appropriate medium, very often until stationary phase to obtain as much biomass as possible, then harvested by centrifugation. The reactions catalysed then take place in either a phosphate buffer or an aqueous solution of the compound to be metabolised. Even though some of these processes are used commercially, there are no reports on how the physiological state of the cells, in these cases starvation, may affect the production of the product of interest, the half-life of the catalyst or its renewal. Interestingly, in a chemostat biodesulphurisation study, it was found that highest activity was obtained when Rhodococcus cells were selectively starved for sulphur, but not for carbon or other nutrients (Kishimoto et al., 2000).

For bioremediation, it is essential that the organisms used can survive in low-energy environments, particularly soil. It has been shown that polyurethane-immobilised Rhodococcus could survive for more than six months in soil, and that they increased the 
degradation rate of pentachlorophenol (Briglia et al., 1990). Autochthonous populations of Rhodococcus were found in soil after a controlled oil spill, even though they could not be detected before the oil spill (Peressutti et al., 2003). This suggests that the population naturally present in soil was growth-restricted, but that it retained the ability to grow and degrade hydrocarbons. Rhodococcus species could be found in soil for up to 13 months after the oil spill, also showing that they successfully competed with other species present in the ecosystem (Peressutti et al., 2003). Finally, in a bioremediation study of activated sludge with phenol-degrading Ralstonia eutropha, it was found that cells that were starved prior to addition to the sludge survived longer than exponential phase cells (Watanabe et al., 2000). Although this type of protocol has not been investigated with Rhodococcus, it could point to an easy and effective way of increasing the efficiency of bioremediation. 


\subsection{Pathogenicity of Rhodococcus}

\subsubsection{Rhodococcus equi infections: epidemiology and disease}

Due to its pathogenic nature, Rhodococcus equi is probably one of the best studied Rhodococcus species so far. It was isolated for the first time from foals and humans in 1923 and in 1967, respectively. It was first classified as a Corynebacterium, then assigned to the Mycobacterium genus and finally reclassified as Rhodococcus (Mosser $\&$ Hondalus, 1996). It has attracted attention because it is the main cause of pneumonia in foals between 1 and 6 months old leading to fatality, and, more recently, due to its emergence as a human opportunistic pathogen.

In foals, the most common clinical manifestation of infection by $R$. equi is a chronic and suppurative pneumonia, with extensive abscessation and lymphadenitis. Intestinal manifestations are present in about $50 \%$ of all foals with pneumonia. The intestinal infection is characterised by ulcerative enterocolitis that can lead to peritonitis, which is usually fatal. It is especially difficult to diagnose since the symptoms are subtle and complex until rather extensive damage has been done to the lung. Joint inflammation, with or without infection can occur, as well as osteomyelitis and subcutaneous lesions (for a review, see Giguère \& Prescott, 1997). In adult horses, disease due to $R$. equi is rare and has been associated with immunodeficiency (Freestone et al., 1987). Furthermore, although rare, $R$. equi has been found to cause disease in other animals such as cats, pigs, cattle and dogs (Fairley \& Fairley, 1999; Hondalus, 1997).

In humans, $R$. equi is associated with immunocompromisation, including in individuals with AIDS and patients undergoing immuno-suppressive therapy (Prescott, 1991; Takai et al., 1994). The predominant clinical manifestation is pneumonia, but other infections 
reported include subcutaneous lesions, osteomyelitis, pericarditis and skin infections. Recurrence is common, and the treatment is long, about six months. The same treatment is applied to horses and humans, and generally consists of a combination of erythromycin and rifampin (Giguère \& Prescott, 1997; Perez et al., 2002). The mortality rate is about $20-25 \%$ in immunocompromised patients, but over $50 \%$ for HIV patients (Perez et al., 2002).

\section{Virulence determinants of Rhodococcus equi:}

In vitro experiments have shown that $R$. equi binds to the macrophage complement receptor CR3 (Hondalus et al., 1993). The bacteria are phagocytosised but the phagosome-lysosome fusion is inhibited (Zink et al., 1987). R. equi can therefore replicate in the phagosome, explaining why the treatment of choice includes antibiotics that concentrate in macrophages. Similar behaviour has been observed for other intracellular pathogens such as Mycobacterium, Nocardia asteroides and Legionella pneumophila. Interestingly, avirulent strains have been shown to persist in macrophages but are incapable of multiplying (Hondalus \& Mosser, 1994). For a review, see Hondalus (1997).

The main virulence factors of $R$. equi identified to date are plasmid-encoded proteins. All virulent strains isolated from foals contain a plasmid in the 80-90 kb range (Makrai et al., 2002). This plasmid harbours the gene for the $15-17 \mathrm{kDa}$ VapA protein (virulence-associated protein), a cell-surface protein of unknown function (Takai et al., 1991). Sequence analysis of the virulence plasmid revealed a putative pathogenicity island harbouring the vapA gene and six additional vap genes (vapC, $D, E, F, G$ and $H$ ). Deletion and complementation analyses provided clear evidence for VapA as a virulence factor in R. equi (Jain et al., 2003). In contrast to foal-derived clinical isolates, 
all of which harbour an 80-90 kb VapA-encoding plasmid, the plasmid profile of human isolates is more diverse. Isolates from immunocompromised patients without AIDS were mostly avirulent and plasmid-free, while isolates from AIDS patients harboured a virulence plasmid of $79-100 \mathrm{~kb}$ with a degree of relatedness to that harboured by equine isolates (Takai et al., 1994; Takai et al., 1995).

Virulence plasmid gene expression was recently analysed using a DNA microarray containing 66 out of the 69 identified ORFs from the $80 \mathrm{~kb}$ virulence plasmid (Ren \& Prescott, 2003). A comparison of in vivo and in vitro growth revealed that, in macrophage-grown $R$. equi, all seven vap genes were induced, in addition to 6 ORFs of unknown function located in the putative pathogenicity island (PI). The observation of a putative IdeR binding site upstream of vapA is significant since IdeR controls transcription of genes encoding proteins contributing to bacterial survival within macrophages, as well as proteins for iron uptake and sequestration in Mycobacterium tuberculosis (Gold et al., 2001). Furthermore, an IdeR homologue has been identified in R. equi (Boland \& Meijer, 2000). Investigation of the effect of iron depletion on gene expression revealed the up-regulation of vapA, $C, G$ and $H$ and down-regulation of $v a p D$, suggesting that vapA is part of the IdeR regulon in $R$. equi (Ren \& Prescott, 2003).

Although $R$. equi infection is rarely fatal, the costs associated with treating the disease are high, especially since infected foals are less likely to race once adult (Ainsworth et al., 1998). This has led The Horserace Betting Levy Board, UK, to fund the sequencing of the complete genome of $R$. equi 103S (see www.sanger.ac.uk/Projects/R_equi/). 


\subsubsection{Rhodococcus fascians infections}

The only other Rhodococcus known to be pathogenic is Rhodococcus fascians. Fasciation of sweet pea was first described in 1927, and the causative agent identified in 1936. Its classification remained problematic until the 1980s, when it was finally named Rhodococcus fascians. It is the only known phytopathogen of the genus (Bell et al., 1998; Finnerty, 1992). R. fascians initiates the formation of leafy galls that consist of centres of shoot amplification and shoot growth inhibition. It has a very broad host range, across 39 plant families and 86 genera (Goethals et al., 2001).

Both R. equi and R. fascians are commonly found in soil (Maes et al., 2001; Muscatello et al., 2006). They must therefore survive large variations in temperature, humidity, $\mathrm{pH}$ and other physico-chemical characteristics of soil, as well as the intra-macrophage (for $R$. equi) and plant environment (for $R$. fascians). Soil is widely considered to be oligotrophic (Morita, 1993), so these organisms must have some ways of surviving periods of low energy availability. 


\subsection{Mechanisms of starvation survival in bacteria}

\subsubsection{Definitions}

In this study, we propose to investigate some physiological responses of rhodococci to starvation, and aim to identify molecular elements that participate in the starvation survival of rhodococci. The term "starvation" describes environmental conditions in which bacteria stop growing, or grow at a very slow rate, because of the lack of nutrients. It is also commonly called "stationary phase", although the stationary phase is not necessarily induced by a lack of nutrients.

Starvation survival has been investigated in Vibrio, Escherichia coli, Pseudomonas, Ralstonia, Rhizobium, Sinorhizobium and Salmonella to name a few Gram-negative bacteria (Jenkins et al., 1988; Kjelleberg et al., 1993; O'Neal et al., 1994). Among the non-sporulating Gram-positives, Staphylococcus, Enterococcus, Streptococcus, Listeria, Micrococcus as well as Lactococcus have been studied to a limited extent (Besnard et al., 2000; Clements \& Foster, 1998; Duwat et al., 2000; Giard et al., 1997; Mukamolova et al., 1998; Trainor et al., 1999). Little work has been done to date on Rhodococcus although some work has been done on the close relative Mycobacterium, due to its pathogenicity and its ability to remain dormant in the lung (Betts et al., 2002; Kamalakannan et al., 2002; Shleeva et al., 2002).

It has been suggested that bacteria can enter a state where they are "viable but non culturable" (VBNC), meaning that they still have metabolic activities but cannot multiply (see e.g. Besnard et al., 2000). This definition seems somewhat misnamed as some VBNC bacteria have been reported to be "resuscitated" (McDougald et al., 1998), usually by using special growth conditions, adding nutrients, or mediated through an 
extracellular protein (Mukamolova et al., 1998). It has been suggested that the term "dormant" describes the state of the cell more accurately, as these cells can return to a "viable and cultivable" state (Kell et al., 1998; Kell \& Young, 2000). The following definitions are more precise:

\begin{tabular}{l|l}
\hline $\begin{array}{l}\text { Physiological } \\
\text { state }\end{array}$ & \multicolumn{1}{c}{ Phenotype } \\
\hline $\begin{array}{l}\text { Viable } \\
\text { (culturable) }\end{array}$ & $\begin{array}{l}\text { Capable of division; will form a colony on agar plate or } \\
\text { proliferate observably in liquid medium }\end{array}$ \\
\hline Dormant & $\begin{array}{l}\text { In a state of low metabolic activity and unable to divide or } \\
\text { to form a colony on an agar plate without a preceding } \\
\text { resuscitation phase }\end{array}$ \\
$\begin{array}{l}\text { Non-viable } \\
\text { (non- } \\
\text { culturable) }\end{array}$ & $\begin{array}{l}\text { Incapable of division; will not form a colony on an agar } \\
\text { plate nor proliferate observably in liquid medium }\end{array}$ \\
\hline
\end{tabular}

Table 1.1. Reproduced from Kell \& Young (2000).

\subsubsection{Physiology of starvation}

The physiological responses of non-sporulating bacteria to starvation are extraordinarily similar from species to species, especially in the case of carbon starvation. One of them is the formation of starvation and multiple-stress resistant microcells. The formation of multiple-stress resistant cells has been observed in all bacteria studied so far. Although morphology is not a good marker of starvation, it should be noted that most bacteria change shape, becoming coccoid or round, and dramatically reduce in size, up to $90 \%$ in the case of Vibrio (Baker et al., 1983; Kjelleberg et al., 1993). The aspect of the culture itself may change too, from smooth to rough colonies on agar or clumping and biofilm formation in broth. The rugose and biofilm phenotypes are associated with the production of exopolysaccharides in response to nutrient starvation (Wai et al., 1998). The biofilm is believed to trap and adsorb nutrients and protect bacteria from predators, thus providing a more favourable environment for growth (Wai et al., 1998). 
A model of starvation has been developed with Vibrio, and has subsequently been supported by studies with other organisms. In this model, bacteria adapt to starvationinduced growth arrest by a complex pattern of regulation that turns on the expression of some genes and turns off others (Kjelleberg et al., 1993). This adaptation takes place in three phases during multiple-nutrient starvation in vitro. The first phase is described as a stringent control phase marked by decreased rate of macromolecular synthesis, the temporary accumulation of guanosine-tetraphosphate (ppGpp) and an increased rate of protein degradation. In Vibrio the stringent phase takes place as soon as the cells face carbon deficiency in the medium and lasts about half an hour (Nyström et al., 1990). During the second phase larger events take place, including changes in membrane fatty acid composition. The reserve material poly- $\beta$-hydroxybutyrate (PHB) is degraded and multiple stress resistance to heat, high and low $\mathrm{pH}$ and oxidative damage appears (Nyström et al., 1992). This phase extends for up to 6 hours (Kjelleberg et al., 1993). From then on, the bacteria enter the third phase, which appears as a slow shutdown of metabolic functions in which the rates of respiration and of RNA, protein and peptidoglycan synthesis plummet (Holmquist \& Kjelleberg, 1993).

It was found for Vibrio that only starvation for carbon led to the formation of viable multiple-stress resistant cells, as opposed to cells starved for nitrogen or phosphorus (Holmquist \& Kjelleberg, 1993; Nyström et al., 1992). It was shown that carbon starvation could induce the same physiological changes as multiple-nutrient starvation. Comparison of the protein profile, by 2D gel electrophoresis, of cultures starved for carbon, nitrogen, phosphorus and multiple-nutrient starvation proteins showed that few proteins were specific to carbon starvation (Nyström et al., 1992). In these studies, the 
carbon source was also the energy source, which would indicate that energy is the most important factor in triggering the starvation survival response.

\subsubsection{Molecular components of regulation of the Starvation Survival Response (SSR) in Gram-negative bacteria}

\subsubsection{The stringent response: ppGpp}

In response to a non-growing state a phenomenon called the stringent response is initiated resulting in the accumulation of the "alarmone" guanosine $3^{\prime}, 5^{\prime}$ bispyrophosphate (ppGpp). This alarmone has been identified in all the organisms studied so far, including E. coli, Salmonella, and several Gram-positive bacteria (see below) (Gallant et al., 1972; Spector, 1998). ppGpp is usually present at low concentrations in the cell, its level being stabilised by two enzymes, RelA and SpoT (Hernandez \& Bremer, 1991; Xiao et al., 1991). RelA is the ribosome-associated ppGpp synthetase I and is activated by uncharged tRNAs as a result of the lack of amino acids. SpoT is a bifunctional enzyme with both weak (p)ppGpp synthetic and hydrolytic properties. The lack of amino acids and starvation for a carbon or energy-source inhibit its hydrolytic activity, leading to a rapid increase in the level of ppGpp in the cell (Murray \& Bremer, 1996).

The effects of ppGpp are numerous. High levels of ppGpp block DNA replication at the origin by repressing transcription of $d n a A$, the gene that encodes the initiator protein DnaA (Chiaramello \& Zyskind, 1990). Although ppGpp levels were also observed to have an effect on chromosomal replication in Bacillus subtilis, replication was initiated but then blocked 100-200 kb downstream of the origin. Replication resumed at the blocked site once the stringent response was lifted. Above all, ppGpp influences the transcription of $r p o S$, encoding the sigma factor RpoS which in turn leads to an increase 
in the expression of many starvation inducible genes (for a review, see Hengge-Aronis, 2002a).

\subsubsection{The stationary phase sigma factor: $\sigma^{\mathrm{S}}$}

RpoS is the stationary phase sigma factor, also called $\sigma^{\mathrm{S}}$ or $\sigma^{38}$. The vegetative $\sigma$ factor, $\sigma^{70}(\mathrm{RpoD})$, is the general "house-keeping factor" that recognises transcription initiation signals on DNA. In exponentially growing cells, low levels of RpoS are present, although there are relatively high levels of $r p o S$ mRNA. As the growth rate decreases and the culture enters stationary phase, $r p o S$ transcription is increased 5- to 10-fold, and RpoS levels increase dramatically (Lange \& Hengge-Aronis, 1994). When the concentration of RpoS increases in the cell, it displaces $\sigma^{70}$ from the RNA polymerase, thereby diminishing the activity of $\sigma^{70}$. Both in vitro and in vivo work also showed that ppGpp decreased the ability for wild-type $\sigma^{70}$ to compete with $\sigma^{\mathrm{S}}$ and bind to the core RNA polymerase (Jishage et al., 2002). RpoS has been identified in E. coli and is present in the branch of proteobacteria. It is recognised as the key regulator of the general stress response. Regulation of RpoS occurs at every level: transcriptional, translational and post-translational. For a review, see Hengge-Aronis (2002b).

A number of factors have been implicated in the control of $r p o S$ transcription, including ppGpp, polyphosphate and cAMP-CRP. Quorum-sensing systems have also been implicated in the transcriptional regulation of $\operatorname{rpoS}$ (Lazazzera, 2000). ppGpp and polyphosphates have a part in $r p o S$ regulation and RpoS activity at three different points in the pathway; they increase the transcription of $\operatorname{rpoS}$, stimulate the activation of inactive $r p o S$ mRNA into active mRNA that can be translated, and enhance the binding of $\sigma^{\mathrm{S}}$ to the RNA polymerase core enzyme (Figure 1.2) (Al-Maghrebi \& Benov, 2001; Hengge-Aronis, 2002b). This is supported by the observation of E. coli mutants lacking polyphosphate kinase (PPK) or RpoS. ppk mutants are unable to accumulate 
polyphosphates and demonstrate reduced viability in stationary phase (nutrient deprivation) and are less resistant to heat, oxidative and osmotic stress (Rao \& Kornberg, 1996). Interestingly, introduction of extra copies of $\operatorname{rpoS}$ on a multi-copy plasmid restored the heat resistance of the ppk mutants to that of the wild-type strain. Furthermore, E. coli rpoS ${ }^{-}$lacked polyphosphate protection against oxidative DNA damage (Al-Maghrebi \& Benov, 2001).

A complex network of proteins and regulatory RNAs is involved in the activation of rpoS mRNA (see Figure 1.2), some of which are induced at late log phase by ppGpp and by oxidative stress (HU, histone-like protein; Hfq, host factor for coliphage Q $\beta$ and DsrA, small RNA regulator of transcription). UDP-glucose, whose concentration increases in the presence of glucose or galactose, decreases the translation levels of RpoS, although the level at which it acts, its mode of action and whether its cellular levels changes during stress or starvation is unknown. Histone-like protein H-NS is activated by cold and inhibits HU, Hfq and DsrA, as well as the activation of rpoS mRNA. Transcription of regulatory RNA OxyS is induced by oxidative stress, including $\mathrm{H}_{2} \mathrm{O}_{2}$, and it subsequently reduces the transcription of rpoS RNA by binding to and inhibiting Hfq. Factors identified to date involved in translational regulation of RpoS include changes in rpoS mRNA secondary structure. Theoretical predictions of the rpoS mRNA secondary structure have been made, although the in vivo structure remains to be determined (Hengge-Aronis, 2002b). 


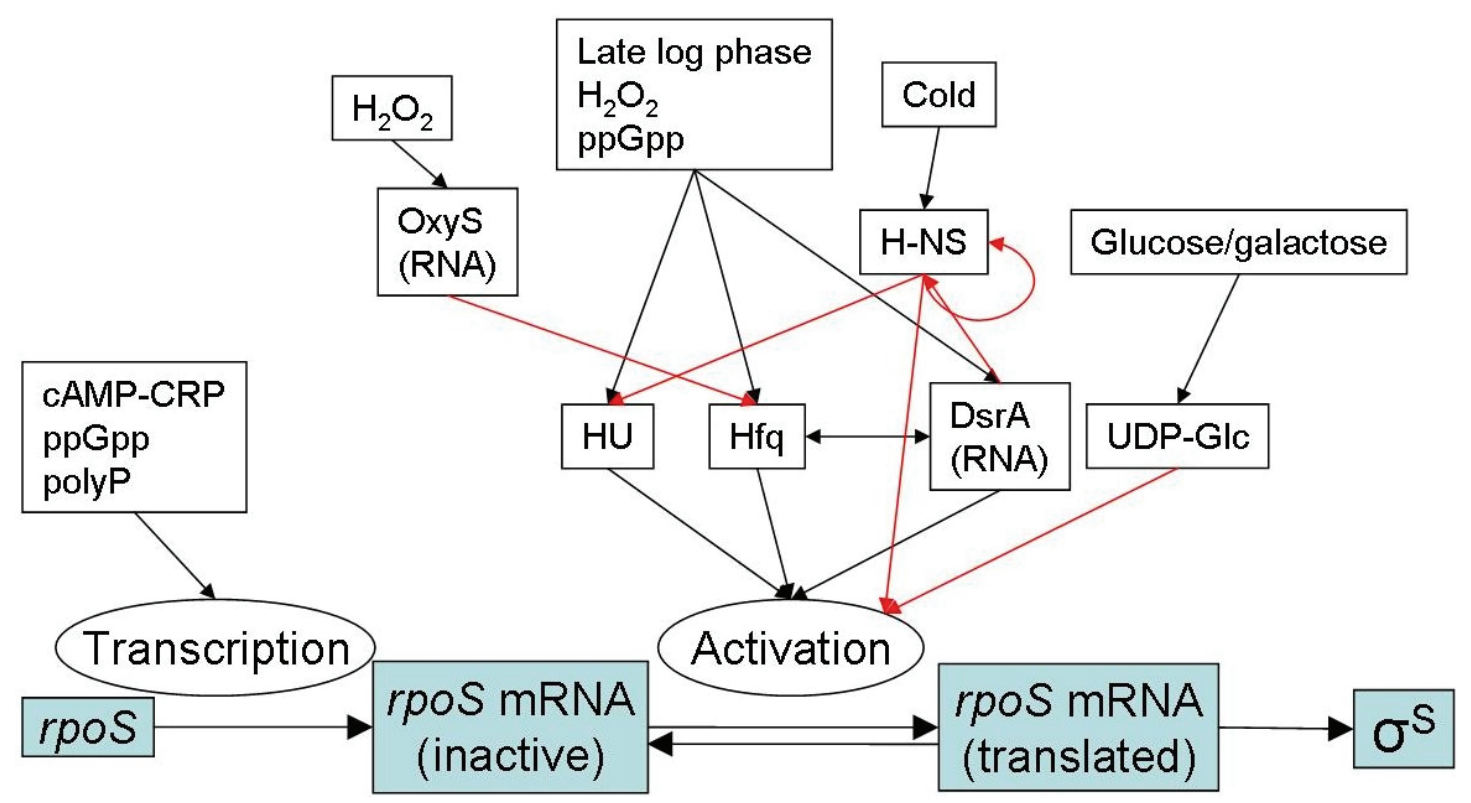

Figure 1.2. Transcriptional and translational regulation of $r p o S$, leading to synthesis of $\boldsymbol{\sigma}^{\mathbf{S}}$. Black arrows, induction; red arrows, repression. See text for details. Adapted from Hengge-Aronis (2002b).

cAMP-CRP, cAMP-receptor protein; ppGpp, guanosine 3', 5'-bispyrophosphate; polyP, polyphosphate; HU, histone-like protein; Hfq, host factor for coliphage Q $\beta$; H-NS, histone-like protein; DsrA, small RNA regulator of transcription; OxyS, regulatory RNA induced by oxidative stress. 
A number of small regulatory RNA molecules targeting rpoS translation have been identified in E. coli and include DsrA, OxyS and RprA (reviewed by Hengge-Aronis, 2002b). DsrA plays a role in low-temperature induction of $r p o S$ translation (Sledjeski et al., 1996), while OxyS is induced by oxidative stress (Altuvia et al., 1997). RprA also stimulates RpoS translation, but the physiological conditions by which it responds have yet to be determined.

In addition to transcriptional and translational control, control of RpoS activity is also possible through regulated proteolysis by the ClpXP protease. RssB is a targeting factor which, when phosphorylated, exhibits specificity for RpoS and thereby tags RpoS for proteolysis by the ClpXP protease (see Figure 1.3) (Jenal \& Hengge-Aronis, 2003; Weichart et al., 2003). Production of RssB is controlled by $\sigma^{\mathrm{S}}$. The integration of all these signals to control $\sigma^{\mathrm{S}}$ remains to be elucidated. 


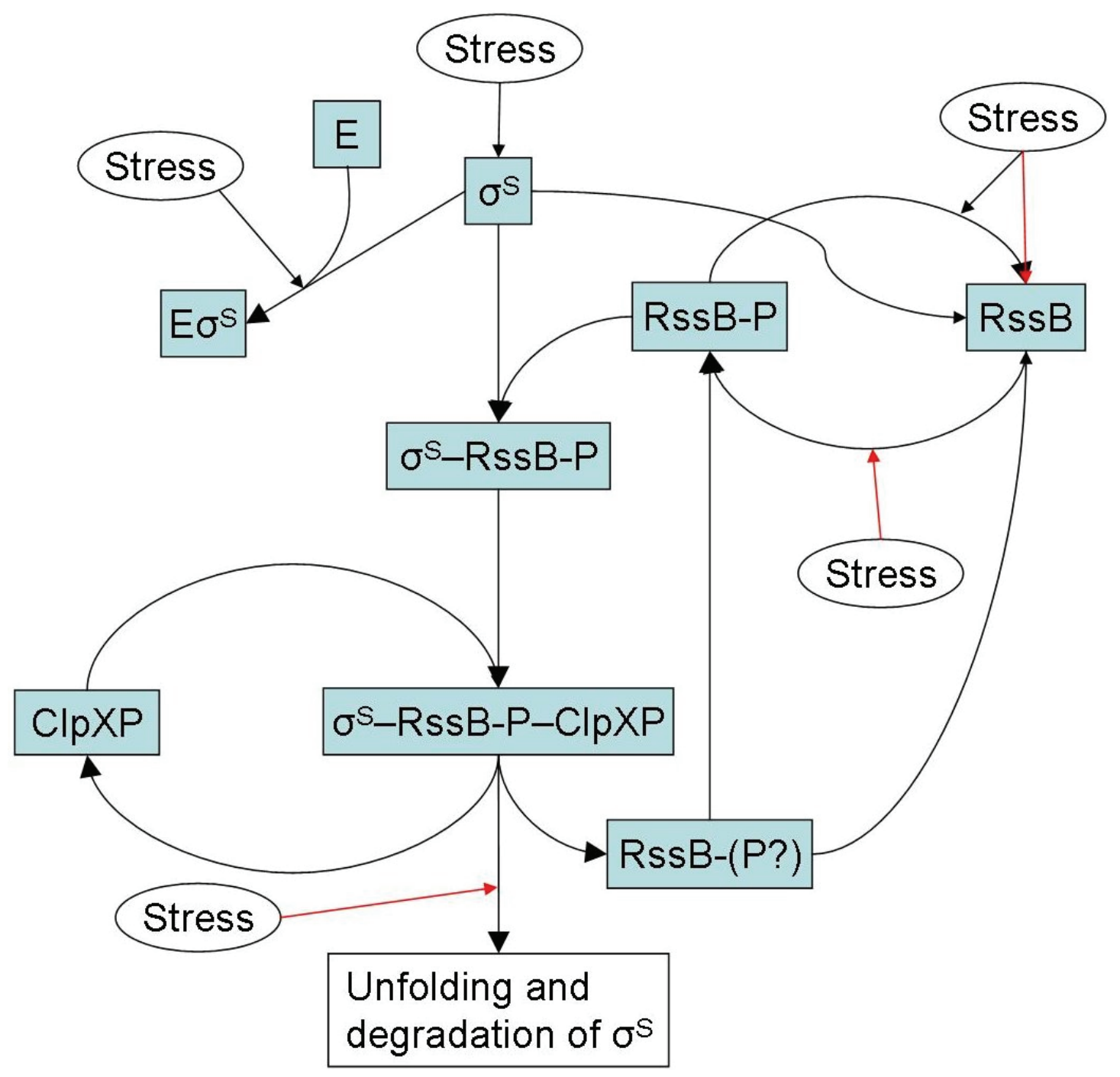

Figure 1.3. Recognition and degradation pathway of $\boldsymbol{\sigma}^{\mathbf{S}} \cdot \sigma^{\mathrm{S}}$ displaces $\sigma^{70}$ from RNA polymerase (E), leading to expression of a different transcriptome. Which stresses intervene at each stage of the pathway has not yet been determined, but the half-life of $\sigma^{\mathrm{S}}$ is known to increase under sudden carbon starvation, heat shock, osmotic upshift and acidic $\mathrm{pH}$. Black arrows, induction; red arrows, repression. Adapted from HenggeAronis (2002b).

E, RNA polymerase; ClpXP, ATP-dependent protease consisting of proteolytic (ClpP) and chaperone (ClpX) subunits; RssB, two-component-type response regulator. 


\subsubsection{Gene expression controlled by $\sigma^{\mathrm{S}}$}

It is estimated that the expression of more than 70 genes is affected by $\sigma^{\mathrm{S}}$, encoding functions leading to altered morphology and metabolism, increased survival during starvation and fluctuating environmental conditions, and also factors leading to increased virulence and cell death (Hengge-Aronis, 1993; Hengge-Aronis, 2002b). Many of the RpoS-dependent genes are not required during the early stationary phase, but provide protection for the cell when de novo protein synthesis might not be possible. The $\operatorname{sts} A$ and $o t s B$ genes, for example, encode enzymes required for production of the osmoprotectant trehalose. The regulation of these genes is RpoS-dependent and they are induced in response to osmotic shift during the exponential phase (Hengge-Aronis, 1996) and during entry into stationary phase (Hengge-Aronis et al., 1991).

Microarray analysis of the whole genome of E. coli K-12 has shown that up to 481 genes are up-regulated by RpoS during transition from exponential to stationary phase (Weber et al., 2005). Of this grand total, 252 were found to be up-regulated during transition to stationary phase, 422 in response to osmotic challenge and 197 in response to acid stress. Interestingly, 140 genes were always up-regulated by RpoS, independently of the challenge. Of those 140 genes, 16 are involved in stress protection (heat, osmotic pressure, oxidative damage etc.), 26 are involved in central energy metabolism (glycolysis, fermentation, pentose phosphate shunt), 11 in regulation, 20 in transmembrane transport, 7 in protein processes and finally 60 were of other or unknown function (Weber et al., 2005). In this study however, the transition was from growth in LB, which is a highly complex medium that can sustain high cell densities, as illustrated by the fact that the authors chose an $\mathrm{OD}_{600 \mathrm{~nm}}$ of 4.0 as the transition point between exponential and stationary phase (Weber et al., 2005). 
When E. coli $\mathrm{K}-12$, grown in a chemostat in chemically defined medium with $0.01 \%$ glucose, was compared with exponential-phase E. coli in $0.1 \%$ glucose, 340 genes were found to be up-regulated and 502 down-regulated (increase or decrease by a factor of more than 2) (Franchini \& Egli, 2006). Among these, 181 up-regulated genes increased expression by a factor of more than 5 , and 81 down-regulated genes decreased expression by a factor of more than 10 . Surprisingly few of these were stress resistanceassociated genes (6 up-regulated, 5 down-regulated). The other genes are involved in transport (32 up-regulated, 11 down-regulated), carbon and energy metabolism (30 upregulated, 7 down-regulated), regulation (14 up-regulated, 12 down-regulated), cell structure (7 up-regulated, 9 down-regulated), protein processing (1 up-regulated, 25 down-regulated), amino acid biosynthesis (1 up-regulated, 1 down-regulated), nucleic acid biosynthesis (1 up-regulated, 12 down-regulated), other functions (3 up-regulated, none down-regulated) and unknown or hypothetical functions (56 up-regulated, 15 down-regulated) (Franchini \& Egli, 2006). The differences between this study and that by Weber et al. (2005) are probably due to the different media employed (i.e. chemically defined $v s$ LB) and differences in protocol (chemostat $v s$ batch), as well as different cut-off points for inclusion in up- or down-regulated lists (i.e. 2- and 5-fold change $v s$ 4-fold change in gene expression). 


\subsubsection{Molecular components of regulation of the SSR of Bacillus subtilis}

\subsubsection{Sigma factor, $\operatorname{SigB}\left(\sigma^{\mathrm{B}}\right)$}

In Bacillus subtilis, the secondary sigma factor $\sigma^{\mathrm{B}}$ is a key element in the control of the general stress response (for a review, see Hecker et al., 1996). The $\sigma^{\mathrm{B}}$-dependent general stress regulon encompasses over 100 stress genes differentially expressed in response to glucose, phosphate or oxygen starvation, and heat, alcohol, acid and osmotic stress (Voelker et al., 1995). The mechanism by which $\sigma^{\mathrm{B}}$ is activated has been well studied. In contrast to $\sigma^{\mathrm{S}}$, it does not rely on ppGpp but reacts to the ratio of ATP to ADP in the cell. The $\operatorname{sig} B$ operon comprises eight genes transcribed constitutively from a $\sigma^{\mathrm{A}}$ (housekeeping factor)-dependent promoter upstream of $r s b R$ (Wise \& Price, 1995). There is also a second internal promoter recognised by $\sigma^{\mathrm{B}}$ located immediately upstream of $r s b V$, which leads to increased expression of $r s b V, r s b W, \operatorname{sig} B$ and $r s b X$ (Duncan et al., 1987). Although all proteins encoded by the $\operatorname{sig} B$ operon are probably involved in the regulation of $\sigma^{\mathrm{B}}$ activity, RsbW is the primary regulator.

RsbW can bind either to $\sigma^{\mathrm{B}}$, thereby sequestering it and preventing its activity, or to $\mathrm{RsbV}$, resulting in free $\sigma^{\mathrm{B}}$, which can then activate transcription of $\sigma^{\mathrm{B}}$-dependent genes. The formation of the RsbW-RsbV complex is controlled by RsbW and influenced by RsbU and RsbX (Boylan et al., 1992; Duncan et al., 1987). Scientific evidence to date supports the presence of two independent pathways for activation of $\sigma^{\mathrm{B}}$. In the case of starvation for glucose or phosphate, RsbW activity responds to the ATP:ADP ratio. It has been confirmed for B. subtilis that glucose and phosphate limitations result in a reduction in the intracellular level of ATP (Voelker et al., 1995). In the presence of low concentrations of ATP, RsbW (a protein kinase) cannot phosphorylate RsbV, as a result of which the RsbW-RsbV complex is then formed. Therefore, the $\sigma^{\mathrm{B}}$ factor is free to 
interact with the RNA polymerase and to drive expression from $\sigma^{\mathrm{B}}$-dependent promoters. For reviews, see Hecker et al.(1996) and Hecker and Volker (1998).

Activation of $\operatorname{sig} B$ in response to glucose or phosphate starvation confers multiple-stress resistance to $B$. subtilis through the expression of over 100 genes, involved in heat, oxidative, water and osmotic stress resistance, as well as many with unknown function (Hecker \& Völker, 1998; Völker et al., 1999). Interestingly, characterisation of a sigB mutant revealed that $\sigma^{\mathrm{B}}$, although activated by glucose and phosphate starvation, is not required for starvation survival (Völker et al., 1999). It is therefore believed that $\sigma^{\mathrm{B}}$ provides additional protection to the bacteria against stresses that could arise during long-term starvation.

\subsubsection{Stringent response in Bacillus}

A stringent response mechanism, similar to that in E. coli, has been reported in B. subtilis. A bifunctional RelA enzyme with the activity of the E. coli RelA and SpoT has been isolated from B. subtilis. As in E. coli, this enzyme provokes an increase in intracellular ppGpp levels under nutrient-limited conditions (Wendrich \& Marahiel, 1997). This results in a more than threefold decrease in the expression of translational apparatus genes, including rRNAs, tRNAs, ribosomal proteins (almost all, including nus $A$, rроA and rроB) and translation factors (e.g. tig, inf $A-C$, fus, tufA and tsf) (Eymann et al., 2002). Genes expected to be expressed in actively growing cells (i.e. involved in nucleotide biosynthesis, synthesis of lipids, energy metabolism, RNA modification, protein modification, cell wall synthesis and DNA replication) are also repressed (Eymann et al., 2002). Relatively few transcription units were up-regulated by the stringent response (approximately 50). These include, in particular, the branchedchain amino acid biosynthetic operon (Ile, Val and Leu; ilv-leu) and a $\gamma$-aminobutyrate 
permease, urease, serine proteases and alkaline protease (gabP, ure, vpr and epr, and aprE). This possibly reflects the need to relieve the amino acid-induced starvation response (Eymann et al., 2002). Sporulation-associated genes are also up-regulated (e.g. $y v y D, y t x G H I$, spoOA, and spoVG), presumably so that sporulation can occur quickly under worsening conditions. Additionally, a relA mutant was found to sporulate less efficiently than the wild-type strain (Eymann et al., 2002). The stringent response in Bacillus also results in termination of DNA replication, located approximately 100$200 \mathrm{~kb}$ on either side of oriC. A contrahelicase, RTP (replication terminator protein), prevents further progression of the replisome by binding constitutively to LSTer and RSTer (left and right stringent termini), approximately $100 \mathrm{~kb}$ up- and downstream of the origin of replication on the chromosome. ppGpp does not increase the DNA-binding affinity of RTP. It is interesting to note that, in a mutant lacking RTP, the stringent response retarded the replisome, showing that other mechanisms are involved in stopping replication (Autret et al., 1997).

\subsubsection{Starvation survival response of Staphylococcus aureus}

Staphylococcus aureus is an important human pathogen resulting in diseases ranging from mild skin infection to life-threatening septicaemia. It is of particular concern in hospitals as it is one of the most important causes of nosocomial infection, due to its ability to survive for extended periods of time outside of the host. During this period, S. aureus is able to survive long-term starvation and fluctuating environmental conditions. S. aureus was proven to survive at least 7 days of carbon starvation in in vitro experiments (Clements \& Foster, 1998). Further characterisation of the SSR showed responses similar to those already identified in other bacteria, including reductive cell division, and an increase in resistance to acid shock and oxidative stress (Watson et al., 1998b). In order to further investigate this, Watson et al. (1998a) 
screened a mutant bank for starvation survival deficient mutants (i.e. mutants that were unable to survive starvation-induced stationary phase). Based on sequence analysis, the genes identified encoded a putative superoxide dismutase, a haem A synthase, an RNA polymerase $\sigma$ subunit, an SOS response component and a phosphoribosyltransferase. Further work confirmed the presence of a haem A synthase (ctaA) and a major superoxide dismutase $(\operatorname{sod} A)$ in $S$. aureus and their role in starvation survival (Clements et al., 1999a; Clements et al., 1999b).

Superoxide dismutases (SOD) detoxify superoxide-anions resulting in production of hydrogen peroxide. In many bacteria, including E. coli, Bacillus and Listeria, increased production of SOD has been observed during stationary phase growth (Dukan \& Nyström, 1999; Inaoka et al., 1998; Nyström, 1999; Vasconcelos \& Deneer, 1994). This may be part of a mechanism used by the bacterium to protect proteins from oxidative damage, an important activity considering that there is a low protein turnover due to a low level of de novo protein synthesis. Analysis of the $S$. aureus sod mutant revealed a low level of survival in stationary phase of growth (Watson et al., 1998a). The S. aureus sod mutant demonstrated a survival defect with amino-acid limitation only. During carbon and phosphate limitation it behaved identically to the parent strain (Watson et al., 1998a). The effect of the $\operatorname{sod} A$ mutation during amino acid starvation is dependent on the degree of aeration. When incubated statically the behaviour of the sodA mutant is identical to the parent strain. In contrast, increased aeration results in a dramatic reduction in the viability of the mutants (Clements et al., 1999a). Interestingly, the sodA mutant demonstrated increased sensitivity to acid compared to the wild-type strain, although the role of $\operatorname{sod} A$ in acid resistance remains to be investigated. Using a mouse abscess model to determine the consequence of the sodA defect on pathogenicity, no significant differences were observed between the mutant and wild-type strain. 
The stringent response in $S$. aureus results in a sharp increase in ppGpp intracellular concentration following nutrient (carbon or amino acid) starvation (Crosse et al., 2000). Immunological analysis revealed that $S$. aureus contains a protein exhibiting crossreactivity with $E$. coli anti-RelA and anti-SpoT antibodies.

A gene homologous to the $B$. subtilis $\operatorname{sig} B$ has been identified in $S$. aureus, although the genetic organisation differs, in that the $S$. aureus $\operatorname{sig} B$ locus lacks the upstream $r s b R$, $r s b S$ and $r s b T$ genes (Kullik \& Giachino, 1997; Wu et al., 1996). Analysis of a S. aureus mutant revealed that $\sigma^{\mathrm{B}}$, although not essential for starvation survival, contributes to increased stress resistance (Chan et al., 1998).

\subsubsection{Starvation survival of Mycobacterium}

Mycobacterium tuberculosis is a pathogen that kills over 2 million people every year, and one third of the world population is believed to be currently infected (WHO, 2002). The incidence of M. tuberculosis infection is on the increase in developed and developing countries. One of the main challenges facing control of this pathogen is that M. tuberculosis cells can remain dormant in lung lesion tissue and can reactivate years later, leading to tuberculosis. The mechanisms that enable Mycobacterium to survive for long periods are poorly understood.

In order to gain an understanding of the molecular basis for dormancy, both M. tuberculosis and M. smegmatis, a fast-growing relative, have been studied. M. smegmatis was shown to be able to survive carbon, nitrogen or phosphorus starvation for 650 days in vitro in chemically defined medium (Smeulders et al., 1999). During this time they were shown to undergo reductive cell division and exhibited 
resistance to oxidative, osmotic and acid stress. Following starvation, mycobacterial cells tend to clump, probably as a result of changes in surface characteristics (Shleeva et al., 2002; Smeulders et al., 1999).

Non-culturable cells could be induced in vitro in M. tuberculosis by letting them grow into late stationary phase (4-5 months) in modified Sauton's medium (chemically defined, $6 \%$ glycerol v/v, for details see Shleeva et al., 2002). It is not known whether the cells stop growing because of nutrient exhaustion or cell density. At this stage, the cells lost their culturability (0 CFU for M. tuberculosis) on solid agar, but could be recovered by culture in liquid medium. The recovery was much improved by the addition of filtered spent medium to fresh broth or the addition of Rpf from Micrococcus luteus. Rpf is the resuscitation promoting factor found to resuscitate dormant cells of Micrococcus luteus, and that also stimulates growth of other high $\mathrm{G}+\mathrm{C}$ organisms, including mycobacteria (Mukamolova et al., 1998). Similar predicted genes were found in M. tuberculosis, M. leprae and M. smegmatis, in addition to several other high $\mathrm{G}+\mathrm{C}$ such as Corynebacterium glutamicum and Streptomyces. Rpf from Micrococcus luteus was found to resuscitate M. tuberculosis and $R$. rhodochrous (Shleeva et al., 2002). Further work on putative rpf genes of $M$. avium and M. tuberculosis showed the proteins they encode have similar resuscitating activities to Rpf from Micrococcus luteus (Zhu et al., 2003).

\subsubsection{Stringent response in Mycobacterium}

Homologues of RelA and SpoT were found in a single protein in M. tuberculosis and named $\mathrm{Rel}_{\mathrm{Mtb}}$ (Avarbock et al., 1999). In vitro studies demonstrated that $\mathrm{Rel}_{\mathrm{Mtb}}$ catalyses the synthesis of (p)ppGpp, consistent with the activity of E. coli RelA. The purified $\mathrm{Rel}_{\mathrm{Mtb}}$ also possesses $3^{\prime}$-pyrophosphohydrolase activity, correlating with E. coli 
SpoT activity. This provides further support for the findings that Gram-positive bacteria possess one protein for both synthesis and degradation of (p)ppGpp. Characterisation of a knockout $\mathrm{Rel}_{\mathrm{Mtb}}$ mutant revealed that the mutant grew significantly more slowly in synthetic medium than the wild type strain (Primm et al., 2000). Interestingly, no significant differences were observed for the growth rate of the mutant and wild type in human macrophage-like cell lines (Primm et al., 2000).

\subsubsection{Transcription factors}

The first transcription factor from M. tuberculosis to be associated with the stationary phase is SigF (DeMaio et al., 1996). It has a close homology to SigF and SigB, sporulation and stress-response sigma factors, respectively, from B. subtilis. SigF was shown to be transcribed during stationary phase, nitrogen depletion, cold and alcohol shock, and oxidative stress. Analysis of gene expression in M. bovis BCG (Bacille Calmette-Guérin) strain grown in complex medium revealed strong induction of sigF expression during stationary phase, nitrogen depletion and cold shock. Weak induction of $\operatorname{sig} F$ transcription was observed during the late-exponential phase in response to alcohol shock and oxidative stress. Since no measurements were taken during the latestationary phase, it is not known if $\operatorname{sig} F$ expression is transitional.

In a study of the transcription of $\operatorname{sig} A$ and $\operatorname{sig} B$ in M. tuberculosis (Hu \& Coates, 1999), the level of $\operatorname{sig} A$ mRNA remained constant from early exponential phase through to late stationary phase, and was not affected by stress. The level of $\operatorname{sig} B$ on the other hand was low during exponential phase, increased to its maximum in late-exponential phase and then slowly decreased. Expression of $\operatorname{sig} B$ was also influenced by environmental stress, in that induction of transcription was observed following oxidative stress and heat shock. These results suggest that SigA may be the housekeeping sigma factor, similar to 
$\sigma^{70}$ of E. coli, whereas SigB may be a stationary-phase sigma factor responsive to starvation and environmental stress. SigB and SigF could well work together, each with its own regulon, to ensure survival of dormant cells.

\subsubsection{Mutant, whole-genome and proteome analyses of Mycobacterium - response to starvation}

In a study by Keer et al. (2000), mutants of M. smegmatis were generated by transposon mutagenesis, and screened for starvation survival deficiency, i.e. mutants unable to survive carbon-starvation-induced stationary phase. Five starvation survival deficient mutants were further analysed and the genes disrupted were partly sequenced. Based on bioinformatic studies the predicted genes encoded a putative penicillin-binding protein, a polyketide synthase, a monoamine oxidase, a membrane protein and an enzyme for biosynthesis of biotin.

Disruption mutants of the predicted bioA gene were auxotrophic for biotin, thereby confirming the bioinformatic analysis. Interestingly, mutants harbouring a disruption of a gene encoding a putative membrane protein have altered colony morphology when grown on agar. The isolation of a stationary-phase mutant harbouring a disruption of a gene predicted to encode a putative penicillin-binding protein is supported by observations in E. coli. Penicillin-binding proteins are needed for cell-wall synthesis. Studies of $E$. coli stationary phase cultures indicate an increase in the level of specific penicillin-binding proteins, in comparison with exponential-phase cultures (Dougherty \& Pucci, 1994). The roles of the polyketide synthase and the monoamine oxidase in stationary-phase and starvation survival are unknown.

An interesting study, based on microarray analysis of $M$. tuberculosis starvation, during which the expression of 3649 genes was monitored, has been published (Betts et al., 
2002). Monitoring after 4, 24 and 96 hours of multiple-nutrient starvation (cells washed and resuspended in PBS) revealed that several hundred genes were significantly up- or down-regulated. For example, at 96 hours, 279 and 323 genes were up- and downregulated, respectively (Betts et al., 2002). Up-regulated genes included those involved in antibiotic production and resistance, nucleotide synthesis and metabolism, and sequences related to insertion sequences and phages. In addition to the up-regulation of a number of regulatory genes (e.g. $\operatorname{sig} B, \operatorname{sig} D, \operatorname{sig} E$ and $\operatorname{sig} F$ ), it is interesting to note that the majority of up-regulated genes correspond to proteins with hypothetical or unknown functions (e.g. Rv0116c, encoding a $26.9 \mathrm{kDa}$ protein homologous to a putative secreted protein from $M$. leprae). Down-regulated genes included those involved in the synthesis of amino acids, lipids, prosthetic groups, carriers and cofactors, in addition to genes involved in DNA replication, repair, recombination, restriction and modification, and translation and post-translation modifications. Of the 281 energy metabolism genes, $15 \%$ were significantly down-regulated. In addition, several virulence-associated genes were down-regulated, e.g. the isocitrate lyase required for in vivo survival of $M$. tuberculosis and polyketide pk52 induced during macrophage infection.

Two-dimensional gel electrophoresis analysis was performed in the same study and revealed up-regulation for only three proteins, including an $\alpha$-crystalline homologue and 24.3 and $25.7 \mathrm{kDa}$ hypothetical proteins, of unknown function. The $16 \mathrm{kDa} \alpha-$ crystalline homologue is believed to enhance long-term protein stability, and it was found that only oxygen limitation could increase its synthesis (Yuan et al., 1996).

Four down-regulated proteins were identified, although two of these proteins were recovered in culture filtrates of $M$. tuberculosis. The down-regulated proteins included 
Tig, a chaperone-like protein, and GrpE, which together with DnaJ is a chaperone for DnaK (Pierpaoli et al., 1998). Although few up- or down-regulated proteins were identified in this study, it should be noted that the 2-D gel analysis on the M. tuberculosis proteome was performed on 6-week multiple-nutrient-starved cultures. This is in contrast to the microarray experiment which monitored cultures in early stationary phase (Betts et al., 2002). It is well known that there is a sharp increase in protein synthesis just before the cells enter stationary phase, and therefore these changes were presumably captured in the microarray experiment.

Proteome analysis of a carbon-starved $M$. smegmatis culture identified a Dps homologue which is preferentially expressed under starvation conditions (Gupta et al., 2002). The Dps (DNA-binding protein from starved cells) of E. coli is expressed during stationary phase and then binds non-specifically to the DNA, thereby protecting it against DNase and oxidative damage in particular (Almirón et al., 1992). The Dps-like protein from M. smegmatis (Ms-Dps) was purified and shown to bind non-specifically to DNA. In the presence of Ms-Dps, DNA was protected from oxidative radicals as demonstrated by an in vitro DNA damage assay. The Ms-Dps protein also protected the DNA from DNaseI activity (Gupta \& Chatterji, 2003). Dps-like proteins have been identified in other Gram-positive organisms including B. subtilis and L. innocua (Bozzi et al., 1997; Chen \& Helmann, 1995).

\subsubsection{Starvation survival of Rhodococcus}

Few studies so far have focussed on the starvation survival of Rhodococcus. Most of the information available to date is circumstantial, such as when cells are grown in defined mineral media to stationary phase for accumulation of a biocatalyst (see e.g. Begoña Prieto et al., 2002). Survival of Rhodococcus species has also been demonstrated in 
bioremediation studies using a mixed culture added to polluted seawater (Shkidchenko et al., 2004) as well as in soil (Briglia et al., 1990; Peressutti et al., 2003). Both of these environments are notoriously low in energy sources and have been described as generally oligotrophic (Morita, 1993). Remarkably, Rhodococcus chlorophenolicus was shown to survive for 200 days in soil, all the while degrading polychlorophenol (Briglia et al., 1990). It is also clear from numerous environmental studies that rhodococci are resilient organisms, as they have been isolated from a very wide range of environments, from the deep sea (Heald et al., 2001) to the Antarctic soil (Bej et al., 2000).

In one of the only studies dedicated to the starvation survival of Rhodococcus, $R$. corallinus was shown to recover from total carbon or nitrogen starvation for up to 5 months (Sanin, 2003). Following starvation, when placed in normal growth medium $(0.4 \%$ glucose, w/v), maximum (non-starved) growth rates were achieved after just 5 days in the case of cells that had been starved for carbon, or 10 days if the cells had been starved for nitrogen. The hydrophobicity of the cells was also measured, but little variation was observed (Sanin, 2003; Sanin et al., 2003).

In another study, a large but transient drop in culturability $(5 \operatorname{logs})$ was observed in Rhodococcus rhodochrous by allowing the cells to enter late stationary phase (60 hours growth) in modified Sauton's medium (chemically defined, $6 \%$ glycerol v/v, for details see Shleeva et al., 2002). It is not known whether the cells stopped growing because of nutrient exhaustion or cell density. At this stage, the cells lost their culturability on solid agar, but could be recovered by culture in liquid medium, suggesting that the cells entered a temporary state of non-culturability. Rpf from Micrococcus luteus was found to greatly increase "resuscitation" of "non-culturable" R. rhodochrous (Shleeva et al., 2002). 


\subsection{Objectives}

As described above, the starvation survival of Rhodococcus has not been studied in great detail, in spite of the importance that organisms of this genus hold for industrial processes and bioremediation. Indeed, $R$. erythropolis has been suggested as an important future bioremediation agent (Čejková et al., 2005), and an agent in the biodesulphurisation of fuel (Caro et al., 2007). In addition, with the exception perhaps of $R$. equi, this genus remains relatively unexplored in terms of genetic analyses. Relatively few tools are available for the genetic manipulation of these organisms and complete genome sequences are only now becoming available.

This study consists of two major components. An objective of this research was to study the physiological adaptation of a selected Rhodococcus strain, R. erythropolis SQ1, to energy and carbon starvation, in particular in terms of (i) its capacity to survive starvation, and (ii) the cross-protection to other environmental insults such as heat and oxidative stress, afforded by the transition to nutrient-induced stationary phase.

In addition, the second objective was to generate a mutant bank of $R$. erythropolis SQ1 and to screen this bank for starvation survival mutants, with subsequent sequence analysis of the mutated genes, in order to gain an insight to the molecular mechanisms involved in the starvation survival response of Rhodococcus. 


\section{Chapter II \\ Materials and Methods}




\subsection{Bacterial Strains and Growth Conditions}

The bacterial strains used in this study are described in Table 2.1. Rhodococcus spp. and Escherichia coli were routinely grown overnight in Luria-Bertani (LB) (Atlas, 1996) or Glucose Yeast Extract (GYE) broth on a rotary shaker $(200 \mathrm{rpm})$ and incubated at 27 and $37^{\circ} \mathrm{C}$, respectively. For growth on solid media, $13 \mathrm{~g} / 1$ of bacteriological agar (Oxoid) was added to the broth. Media were sterilised by autoclaving at $121^{\circ} \mathrm{C}$ and 15 pounds per square inch of pressure for $15 \mathrm{~min}$. When required, media were supplemented with antibiotics at concentrations of 200,100 and $40 \mu \mathrm{g} / \mathrm{ml}$ of kanamycin, ampicillin and chloramphenicol (all Sigma-Aldrich), respectively, for Rhodococcus. Final concentrations of $50 \mu \mathrm{g} / \mathrm{ml}$ of kanamycin or $100 \mu \mathrm{g} / \mathrm{ml}$ of ampicillin were used for E. coli. All strains were stocked at -20 and $-80{ }^{\circ} \mathrm{C}$ in $20 \%$ and $40 \%$ glycerol, respectively. 


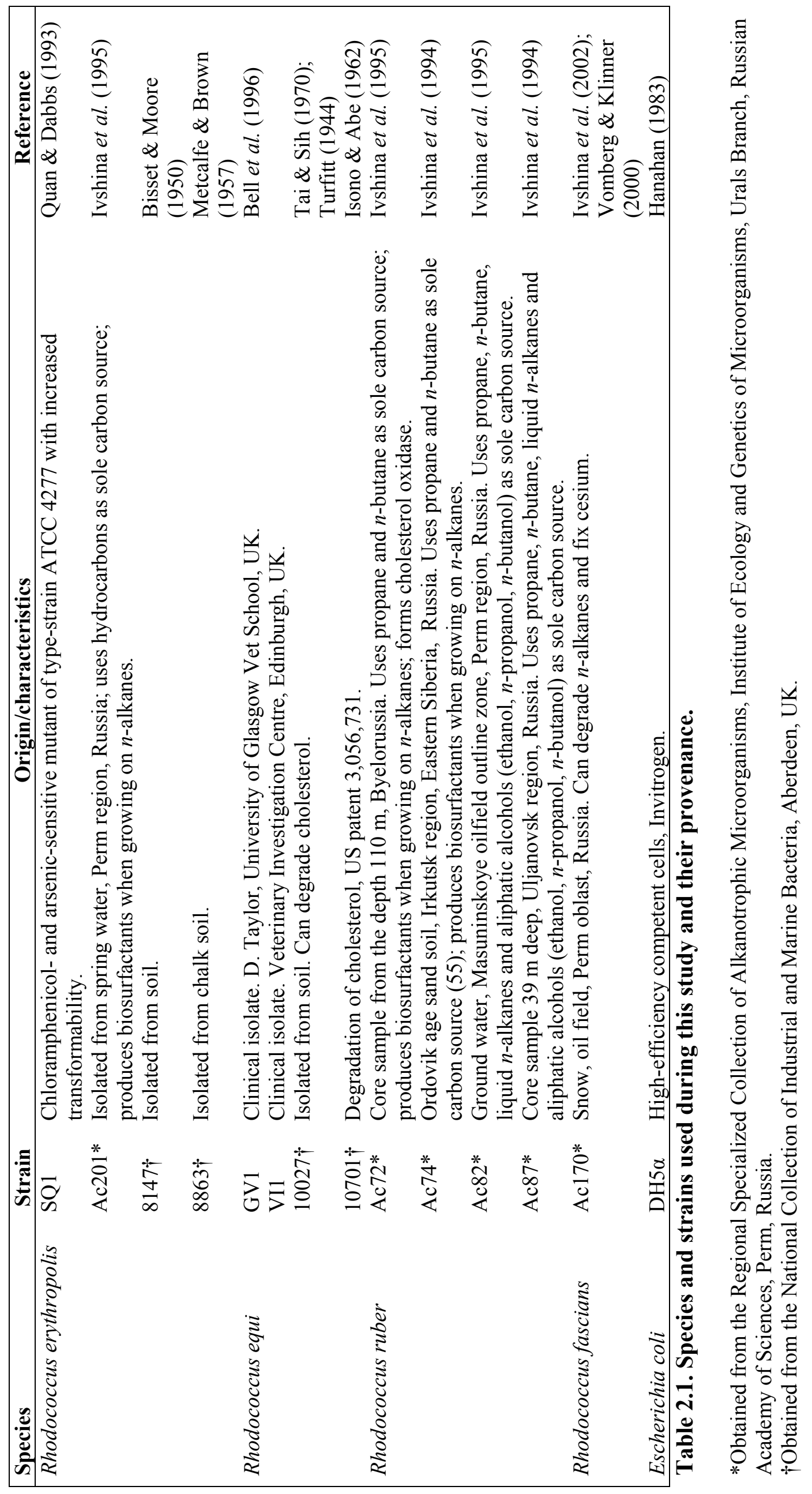




\subsection{Growth curves of R. erythropolis}

Growth curve experiments were performed to establish the growth rate of R. erythropolis SQ1 and to determine when cultures entered stationary phase. Optical density $\left(\mathrm{OD}_{600 \mathrm{~nm}}\right)$ and viable plate count measurements were used to monitor the viability of Rhodococcus and entry into stationary phase. Unless otherwise stated, cultures used to inoculate the medium were in the exponential growth phase. To ensure this, $R$. erythropolis SQ1 stored at $-20^{\circ} \mathrm{C}$ was grown at $27^{\circ} \mathrm{C}$ and $200 \mathrm{rpm}$ in the same medium as that used for the growth curve. When this culture reached an $\mathrm{OD}_{600 \mathrm{~nm}}$ between 0.5 and 1.0 (early exponential phase), it was inoculated at $1 \%(\mathrm{v} / \mathrm{v})$ in the same medium. After overnight growth at $27^{\circ} \mathrm{C}$ and $200 \mathrm{rpm}$, this culture was used to inoculate $100 \mathrm{ml}$ medium (in $500 \mathrm{ml}$ conical flasks) at an $\mathrm{OD}_{600 \mathrm{~nm}} 0.01$. The flasks were then incubated at $27^{\circ} \mathrm{C}$ and $200 \mathrm{rpm}$. Samples were taken periodically to determine the $\mathrm{OD}_{600 \mathrm{~nm}}$ and the viable plate count (Figure 2.1).

To measure the viable plate count, $1 \mathrm{ml}$ of culture was sampled for a serial dilution. Dilutions were performed in $9 \mathrm{ml}$ quarter strength (Ringer) saline supplemented with $0.05 \%(\mathrm{v} / \mathrm{v})$ Tween. Aliquots $(100 \mu \mathrm{l})$ of the appropriate dilutions were then plated in triplicate on LB agar. The plates were then incubated for at least two days at $27^{\circ} \mathrm{C}$ and the viable plate count calculated as colony forming units per $\mathrm{ml}(\mathrm{CFU} / \mathrm{ml})$.

Where specified, glucose concentration in the culture medium was determined during the course of growth. A $1 \mathrm{ml}$ sample of culture was taken and centrifuged at $16,000 \times \mathrm{g}$ for $1 \mathrm{~min}$. The supernatant was then filter-sterilised $(0.22 \mu \mathrm{m}$, Millipore) before storage at $-20{ }^{\circ} \mathrm{C}$. The quantity of glucose in the samples was measured using a colorimetric hexokinase-based method (Glucose HK assay kit; Sigma-Aldrich), following the manufacturer's protocol. The standard curves were done using chemically defined 
medium containing $0.1 \%$ and $1 \%$ glucose. When required, samples were diluted with glucose-free chemically defined medium, so that the only variable would be the glucose concentration. 
R. erythropolis SQ1 stock $\left(-20^{\circ} \mathrm{C}\right)$

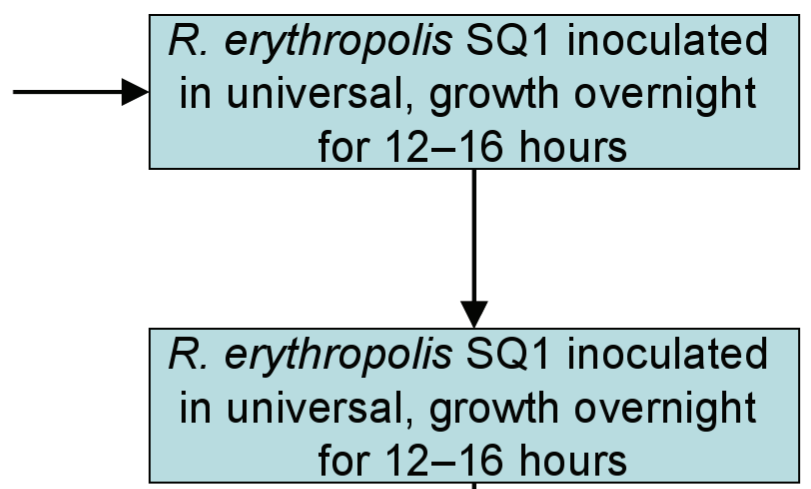

Figure 2.1. Schematic overview of the growth experiments methodology. 


\subsection{Measurement of impact of heat shock on cell viability}

The ability of $R$. erythropolis SQ1 to survive heat shock was evaluated by using a modification of the protocol described by Jenkins et al. (1988). R. erythropolis SQ1 was grown overnight in $100 \mathrm{ml}$ of chemically defined medium (CDM IIA) containing $0.1 \%$ glucose at $27^{\circ} \mathrm{C}$ and $200 \mathrm{rpm}$ to ensure that entry into stationary phase was due to depletion of the carbon source. The inoculum was prepared as described in Section 2.2. The composition of chemically defined medium is provided in Section 2.15. Ten $\mathrm{ml}$ samples were taken at mid-exponential and early-stationary phase. The exponential phase samples were taken after approximately $48 \mathrm{~h}$ of growth at an $\mathrm{OD}_{600 \mathrm{~nm}} 0.5$. Additional samples were taken after 6 days of incubation (early-stationary phase).

The samples were aliquoted $(1 \mathrm{ml})$ in $1.5 \mathrm{ml}$ eppendorfs pre-warmed at $57^{\circ} \mathrm{C}$. The aliquots were then incubated in a $57{ }^{\circ} \mathrm{C}$ waterbath for up to $2 \mathrm{~h}$. At $0,5,10$ and $15 \mathrm{~min}$ one sample was removed from the waterbath and maintained at room temperature for 5 min to cool down. Serial dilutions of the samples were then prepared using quarter strength (Ringer) saline solution supplemented with $0.05 \%(\mathrm{v} / \mathrm{v})$ Tween and $100 \mu \mathrm{l}$ aliquots were plated in triplicate on LB-agar. The plates were then incubated for $48 \mathrm{~h}$ at $27^{\circ} \mathrm{C}$ and the viable count $(\mathrm{CFU} / \mathrm{ml})$ determined. In parallel, an additional late-logphase sample was diluted prior to heat shock treatment to an $\mathrm{OD}_{600 \mathrm{~nm}}$ of approximately 0.5 and a titre of $5.05 \times 10^{8} \mathrm{CFU} / \mathrm{ml}$ (which was reached in mid-exponential phase) in order to determine whether cell density affected the heat resistance profile. Since no difference was observed, the protocol described above was used. 


\subsection{Measurement of impact of oxidative damage on cell}

\section{viability}

Exponential and stationary phase cultures were sampled for investigation of the resistance of $R$. erythropolis SQ1 to oxidative damage. Cells were grown as described in Section 2.2. One $\mathrm{ml}$ samples were taken and the cells harvested by centrifugation at $16,000 \times \mathrm{g}$ for $5 \mathrm{~min}$. The supernatant was discarded and the cells resuspended in quarter strength (Ringer) saline. The washed cells were then used to inoculate $2 \mathrm{ml}$ of quarter strength (Ringer) saline to an $\mathrm{OD}_{600 \mathrm{~nm}}$ of approximately 0.01 (approx. $1 \times 10^{6} \mathrm{CFU} / \mathrm{ml}$ ). The cell concentration was determined by plating three $100 \mu 1$ aliquots. Tert-butyl hydroperoxide (tBHP) was then added to $1 \mathrm{ml}$ of cells at concentrations ranging from 1 to $400 \mathrm{mM}$. To measure cell viability, $100 \mu \mathrm{l}$ samples were taken at regular intervals for serial dilution. Dilutions were done using $900 \mu \mathrm{l}$ quarter strength (Ringer) saline supplemented with $0.05 \%$ Tween. Aliquots $(100 \mu 1)$ of the appropriate dilutions were then plated in triplicate on LB-agar. The plates were subsequently incubated for two days at $27{ }^{\circ} \mathrm{C}$ and the cell viability calculated as colony forming units per $\mathrm{ml}(\mathrm{CFU} / \mathrm{ml})$. 


\subsection{Transformation of cells by electroporation}

\subsubsection{Preparation of electrocompetent cells of $E$. coli and Rhodococcus}

Electrocompetent cells of E. coli DH5a (Invitrogen) were prepared as described in the manual for the Gene Pulser II (Bio-Rad). LB $(100 \mathrm{ml})$ was inoculated with $1 \mathrm{ml}$ fresh overnight culture and grown at $37^{\circ} \mathrm{C}$ with vigorous shaking to an $\mathrm{OD}_{600 \mathrm{~nm}}$ between 0.5 and 1 . The flasks were then chilled on ice for approximately $30 \mathrm{~min}$ and the cells were subsequently harvested by centrifugation at $10,000 \times \mathrm{g}$ for $10 \mathrm{~min}$ in a centrifuge cooled to $4{ }^{\circ} \mathrm{C}$ (5810R, Eppendorf). The supernatant was discarded and the cells were washed twice in ice-cold $10 \%$ glycerol, and then resuspended in $400 \mu 1$ of $10 \%$ glycerol. The cells were aliquoted $(50 \mu \mathrm{l})$ in ice-cold eppendorfs, which were then stored at $-80{ }^{\circ} \mathrm{C}$ until required for electroporation.

A similar protocol was used for the preparation of electrocompetent Rhodococcus but with the following modifications: incubation of the culture at $27^{\circ} \mathrm{C}$ to an $\mathrm{OD}_{600 \mathrm{~nm}}$ of 1.0 and final resuspension of the cell pellet in $2.5 \mathrm{ml}$ of $10 \%$ glycerol (Tanaka et al., 2002).

The protocol described by Zheng et al. (1997) was also used. Briefly, cells for electroporation were prepared by growth of a single colony in $200 \mathrm{ml}$ of LB supplemented with $1.8 \%$ sucrose, $1.5 \%$ glycine and $100 \mathrm{mg} \mathrm{ml}^{-1}$ isoniazid, incubated at $27^{\circ} \mathrm{C}$ with constant shaking at $200 \mathrm{rpm}$ for 2 days, chilling on ice for $1.5 \mathrm{~h}$, and repeated washing in $10 \%$ glycerol dissolved in pure water.

\subsubsection{Electroporation}

An aliquot of competent cells was thawed on ice and gently mixed with 0.5 to $2 \mu \mathrm{g}$ of DNA. The cells were then incubated on ice for approximately $1 \mathrm{~min}$. The electroporator 
(Gene Pulser II with Pulse Controller Plus, Bio-Rad) was set at $25 \mu \mathrm{F}, 2.5 \mathrm{kV}$ and $200 \Omega$ for E. coli (Bio-Rad protocol), or at $25 \mu \mathrm{F}, 2.5 \mathrm{kV}$ and $400 \Omega$ for Rhodococcus (Treadway et al., 1999). The mixture of cells and DNA was transferred to a cold $0.2 \mathrm{~cm}$ electroporation cuvette and placed into the electrotransformation chamber. The sample was pulsed once at the above settings, which should produce a pulse of time constant 4.5-5 ms for E. coli and 8-12 ms for Rhodococcus. The cuvette was removed from the chamber and $950 \mu \mathrm{l}$ of SOC recovery medium (composition of SOC is provided in Section 2.15) (Sambrook et al., 1987) was immediately added. The cell suspension was transferred to a $17 \times 100 \mathrm{~mm}$ sterile propylene culture tube and incubated at $37^{\circ} \mathrm{C}$ and $200 \mathrm{rpm}$ for $1 \mathrm{~h}$ for E. coli. For Rhodococcus, the samples were incubated at $27^{\circ} \mathrm{C}$ and $200 \mathrm{rpm}$ for $2-4 \mathrm{~h}$. The samples were subsequently plated on LB agar plates containing the appropriate antibiotic. 


\subsection{Transposon mutagenesis and generation of mutant bank}

Transposon mutagenesis exploits the ability of transposons to insert randomly into DNA. The EZ-TN $<\mathrm{KAN}-2>$ transposon is an artificial transposon, consisting only of a kanamycin resistance gene from Tn903 flanked by 19 bp inverted repeat mosaic end sequences which serve as binding sites for the transposase (Epicentre Biotechnologies). The DNA is provided as a complex with the EZ-TN transposase enzyme and the resulting transposome can be electroporated into living cells, where the intracellular $\mathrm{Mg}^{2+}$ activates the transposase, leading to random insertion of the transposon into the genome (see Figure 2.2), the only requirement being a TA dimer at the point of insertion (Berg et al., 1983). Mutants are obtained by selection for kanamycin resistance.

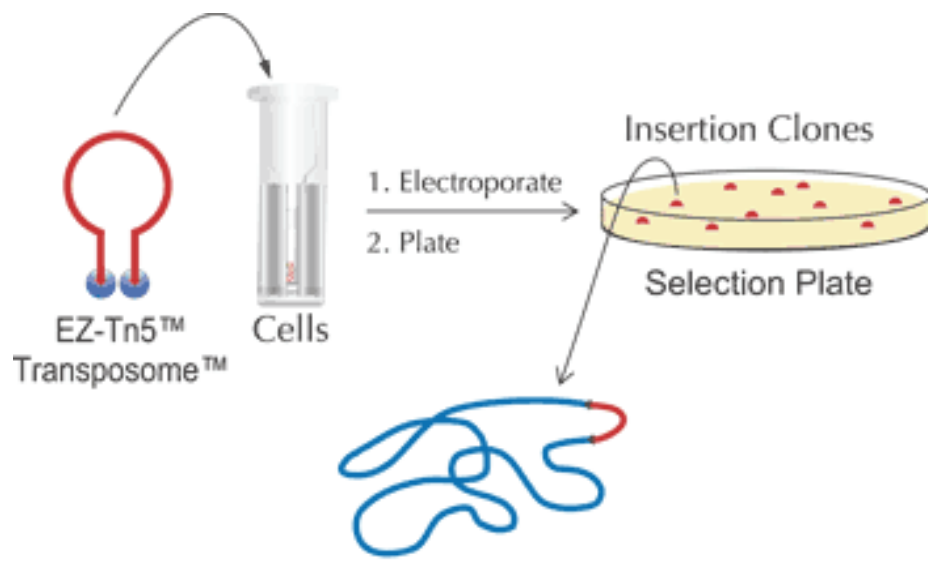

Genomic DNA

Figure 2.2. Generation of a mutant bank by random transposon insertion using the EZ-Tn5 Transposome (@2007 Epicentre Biotechnologies).

Transposon mutagenesis was performed on competent cells of $R$. erythropolis SQ1 prepared as described in Section 2.5.1. Instead of plasmid DNA, $1 \mu$ transposome (transposon-transposase complex) EZ-Tn5 $<$ KAN-2 $>$ Tnp from Epicentre Biotechnologies was used. The electroporated cells were plated on LB agar containing $200 \mu \mathrm{g} / \mathrm{ml}$ of kanamycin and incubated at $27^{\circ} \mathrm{C}$. Each colony growing on the selective 
medium was picked using a sterile toothpick and inoculated in $100 \mu \mathrm{l}$ of LB supplemented with $200 \mu \mathrm{g} / \mathrm{ml}$ of kanamycin in a well of a microtitre plate (stock plates). The plates were incubated overnight at $27^{\circ} \mathrm{C}$ in a static incubator and fresh microtitre plates containing $150 \mu \mathrm{l}$ of $\mathrm{LB}+$ kanamycin per well (working plates) were inoculated using purpose-built sterile 96-prong replicators. $100 \%$ glycerol $(50 \mu 1)$ was added to each well of the stock plates and mixed by pipetting, and the plates were stored at $-80{ }^{\circ} \mathrm{C}$. In each plate, well A1 was inoculated with wild-type R. erythropolis SQ1. Well H12 contained LB only (the non-inoculated negative control). 


\subsection{Mutant screening strategy}

The mutant bank was screened for stationary phase survival mutants following the method described by Uhde et al. (1997). Transfers were made from the working plates to plates containing chemically defined medium CDM IIA (see Section 2.15. for details) containing either $1 \%$ or $0.1 \%(\mathrm{w} / \mathrm{v})$ glucose broth. The working plates were replicated at weekly intervals on $\mathrm{LB}$ agar or CDM $1 \%$ glucose agar to assess the survival of the mutants (see Figure 2.3). The appearance of growth for each mutant was recorded. Further investigation of mutants exhibiting interesting features was continued by going back to the stock plates. Each plate was assigned a number (from 1 to 10) and each well was identified alpha-numerically, so that each mutant has a unique identifying code (e.g. 4C7), allowing easy retrieval of mutants of interest for further analysis. 


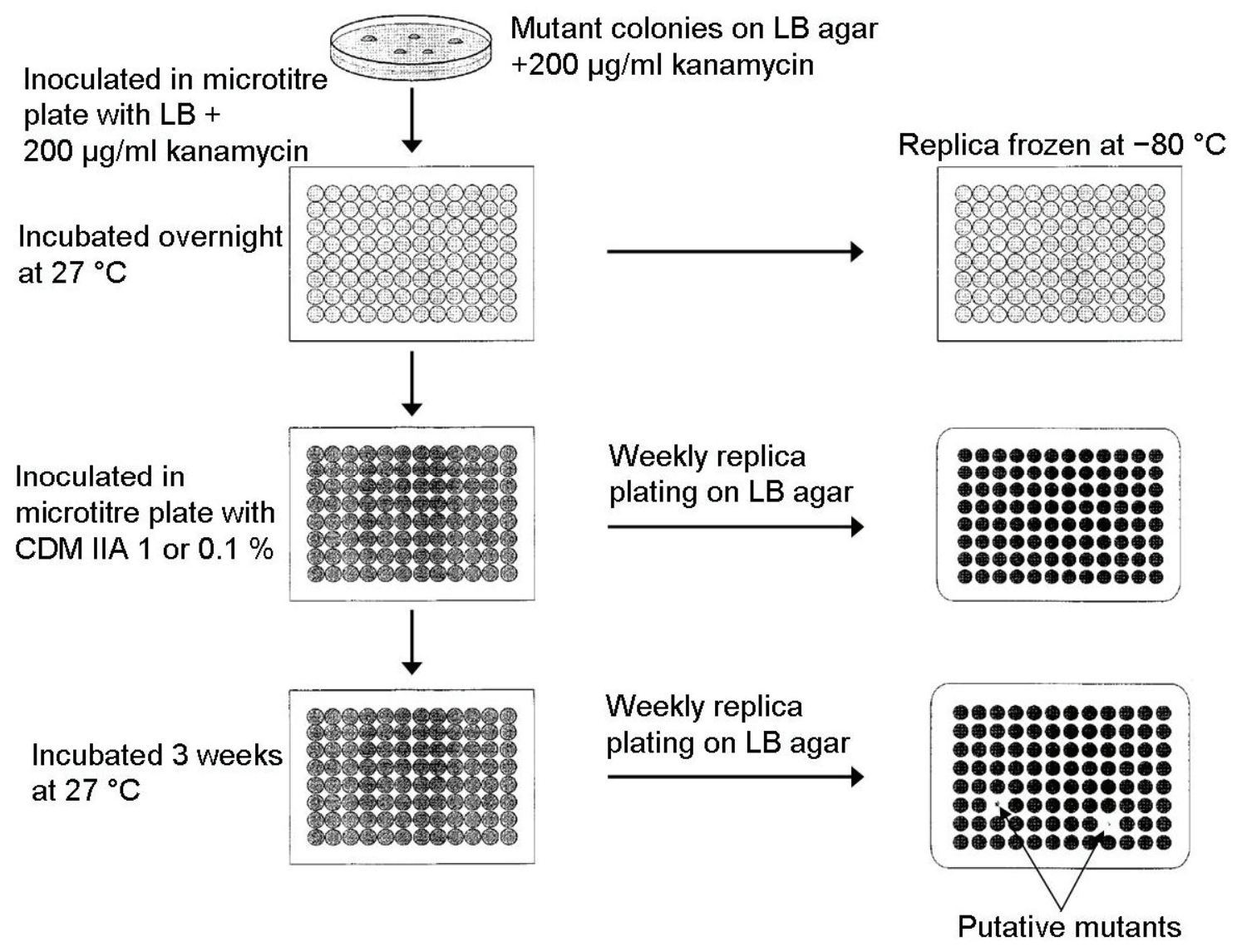

Figure 2.3. Mutant screening strategy (adapted from Uhde et al., 1997). The mutants were not allowed to enter stationary phase until inoculation in CDM II A for screening. 


\subsection{DNA isolation}

\subsubsection{Plasmid DNA Isolation $(<20,000 \mathrm{bp})$}

Plasmid DNA was recovered from bacterial cultures using the Wizard SV Miniprep kit (Promega). For extraction of plasmids from Rhodococcus species the following adaptation was made: 1-5 $\mathrm{ml}$ overnight culture was harvested and resuspended in TE buffer (10 mM Tris-HCl, $1 \mathrm{mM}$ EDTA, pH 8.0) supplemented with $20 \mathrm{mg} / \mathrm{ml}$ lysozyme (Sigma) and incubated for $2-4 \mathrm{~h}$ in a $37^{\circ} \mathrm{C}$ waterbath. The procedure was completed using the DNA isolation kit as per the manufacturer's instructions. The plasmids used in this study are listed in Table 2.2.

\subsubsection{Genomic DNA Isolation}

The FastDNA SPIN Kit for Soil (Qbiogene) was used to extract genomic DNA from $R$. erythropolis SQ1 and mutants thereof. Modifications made to the manufacturer's protocol were as follows. Two to four $\mathrm{ml}$ of broth culture $\left(\mathrm{OD}_{600 \mathrm{~nm}} \sim 1.0\right)$ were centrifuged at $12,000 \times \mathrm{g}$ for $10 \mathrm{~min}$. The pellets were resuspended in $978 \mu \mathrm{l}$ Sodium Phosphate Buffer (SPB) and $122 \mu 1$ MT buffer (provided in the kit), and subsequently transferred to a Lysing Matrix E Tube. The procedure was continued as described in the manufacturer's handbook, including a lysis step at setting 5.5 for $30 \mathrm{~s}$ in the FastPrep Instrument (Qbiogene). In the final step, the genomic DNA was eluted in $100 \mu 1$ DNase- and pyrogen-free water provided in the kit. 


\begin{tabular}{|c|c|c|c|}
\hline Plasmid & $\begin{array}{l}\text { Antibiotic } \\
\text { resistance }\end{array}$ & Description & Reference \\
\hline pRE7 & $\mathrm{Km}^{\mathrm{R}}$ & $\begin{array}{l}\text { E. coli - Rhodococcus shuttle } \\
\text { vector }(5.9 \mathrm{~kb})\end{array}$ & Zheng et al. (1997) \\
\hline pDA71 & $A m p^{\mathrm{R}}, \mathrm{Cm}^{\mathrm{R}}$ & $\begin{array}{l}\text { E. coli - Rhodococcus shuttle } \\
\text { vector }(8.8 \mathrm{~kb})\end{array}$ & Dabbs (1998) \\
\hline pSRK21 & $\mathrm{Km}^{\mathrm{R}}$ & $\begin{array}{l}\text { E. coli }- \text { Rhodococcus shuttle } \\
\text { vector }(5.8 \mathrm{~kb})\end{array}$ & Veselý et al. (2003) \\
\hline pCR2.1-TOPO & $\mathrm{Amp}^{\mathrm{R}}, \mathrm{Km}^{\mathrm{R}}$ & E. coli cloning vector $(3.9 \mathrm{~kb})$ & Invitrogen \\
\hline
\end{tabular}

Table 2.2. Description of the plasmids used in this study.

Amp, ampicillin; Cm, chloramphenicol; Km, kanamycin. 


\subsection{Restriction Analysis of DNA}

Restriction enzymes used were from Roche Applied Science and used according to the manufacturer's instructions. Routinely, the DNA was combined with the appropriate buffer $(10 \times)$ and $10 \mathrm{U}$ of enzyme followed by incubation overnight at $37^{\circ} \mathrm{C}$. When performing multiple digests, the buffer was chosen according to the manufacturer's recommendations in order to yield the highest possible activity.

\subsection{Agarose Gel Electrophoresis}

The results of DNA extraction, DNA restriction analysis and PCR amplification were visualised by agarose gel electrophoresis. Molecular grade agarose $(0.8$ to $1.0 \% \mathrm{w} / \mathrm{v}$, Fluka) was dissolved in Tris-acetate buffer (TAE; $40 \mathrm{mM}$ Tris-base, $20 \mathrm{mM}$ acetate and 2 mM EDTA, pH 8.0) by heating. Ethidium bromide was incorporated in the gel at a final concentration of $0.3 \mu \mathrm{g} / \mathrm{ml}$. The gel was cast in a mould (Embi Tec) and allowed to solidify prior to being submerged in TAE buffer. The molecular size standard used was the New England Biolabs 2-log DNA ladder. The DNA samples were mixed with loading buffer $(0.25 \%$ bromophenol blue, $30 \%$ glycerol in water) prior to gel loading. Samples were electrophoresed through the gel at $150 \mathrm{~V}$ in a Run-One electrophoresis cell (Embi Tec) until the bromophenol blue from the loading buffer had migrated halfway through the gel. Gels were visualised and recorded using the Molecular Imager FX Pro and the Quantity One software (Bio-Rad). When necessary, DNA fragments were extracted from gels using the Montage DNA gel extraction kit (Millipore) following the manufacturer's protocol. 


\subsection{Polymerase Chain Reaction (PCR)}

PCR was routinely performed using BioMix (Bioline), unless otherwise specified. BioMix is a complete reaction mix $(2 \times)$ for PCR that contains $32 \mathrm{mM}\left(\mathrm{NH}_{4}\right)_{2} \mathrm{SO}_{4}$, $125 \mathrm{mM}$ Tris- $\mathrm{HCl} \quad(\mathrm{pH} \mathrm{8.8}), \quad 0.02 \%$ Tween 20, $2 \mathrm{mM}$ dNTPs, $0.05 \mathrm{U} / \mu 1 \quad$ Taq polymerase and $3 \mathrm{mM} \mathrm{MgCl}$. A PCR reaction consisted of $25 \mu 1$ BioMix, $1 \mu 1$ of each primer $(100 \mathrm{pmol} / \mu \mathrm{l})$, DNA template and the volume was adjusted to $50 \mu$ with $\mathrm{dH}_{2} \mathrm{O}$. The primers used in this study are described in Table 2.3. Water was substituted for the DNA template in the negative controls. The reagents were combined and centrifuged briefly prior to being placed in the Px2 thermal cycler (Thermo Scientific). A typical reaction was carried out as follows: a denaturing period of $5 \mathrm{~min}$ at $94{ }^{\circ} \mathrm{C}$, followed by 25 cycles of $1 \mathrm{~min}$ denaturation at $94{ }^{\circ} \mathrm{C}, 1 \mathrm{~min}$ annealing at $50{ }^{\circ} \mathrm{C}$ and $3 \mathrm{~min}$ of elongation at $72{ }^{\circ} \mathrm{C}$. A final elongation period of $10 \mathrm{~min}$ at $72{ }^{\circ} \mathrm{C}$ was then followed by cooling down to $4{ }^{\circ} \mathrm{C}$. The PCR products were then analysed by agarose gel electrophoresis as described above.

\subsection{Inverse $P C R$}

Inverse PCR (iPCR) is a method designed to recover DNA sequences flanking a region of known sequence (Ochman et al., 1988). In this study, iPCR was performed on mutants of $R$. erythropolis SQ1 harbouring the EZ-Tn $<$ KAN2 $>$ transposon (Epicentre Biotechnologies). Genomic DNA was isolated from the mutants and restricted with the appropriate restriction endonucleases, and subsequently religated. The sequence flanking the transposon was then amplified by PCR, as illustrated in Figure 2.4 and described below in further detail. 


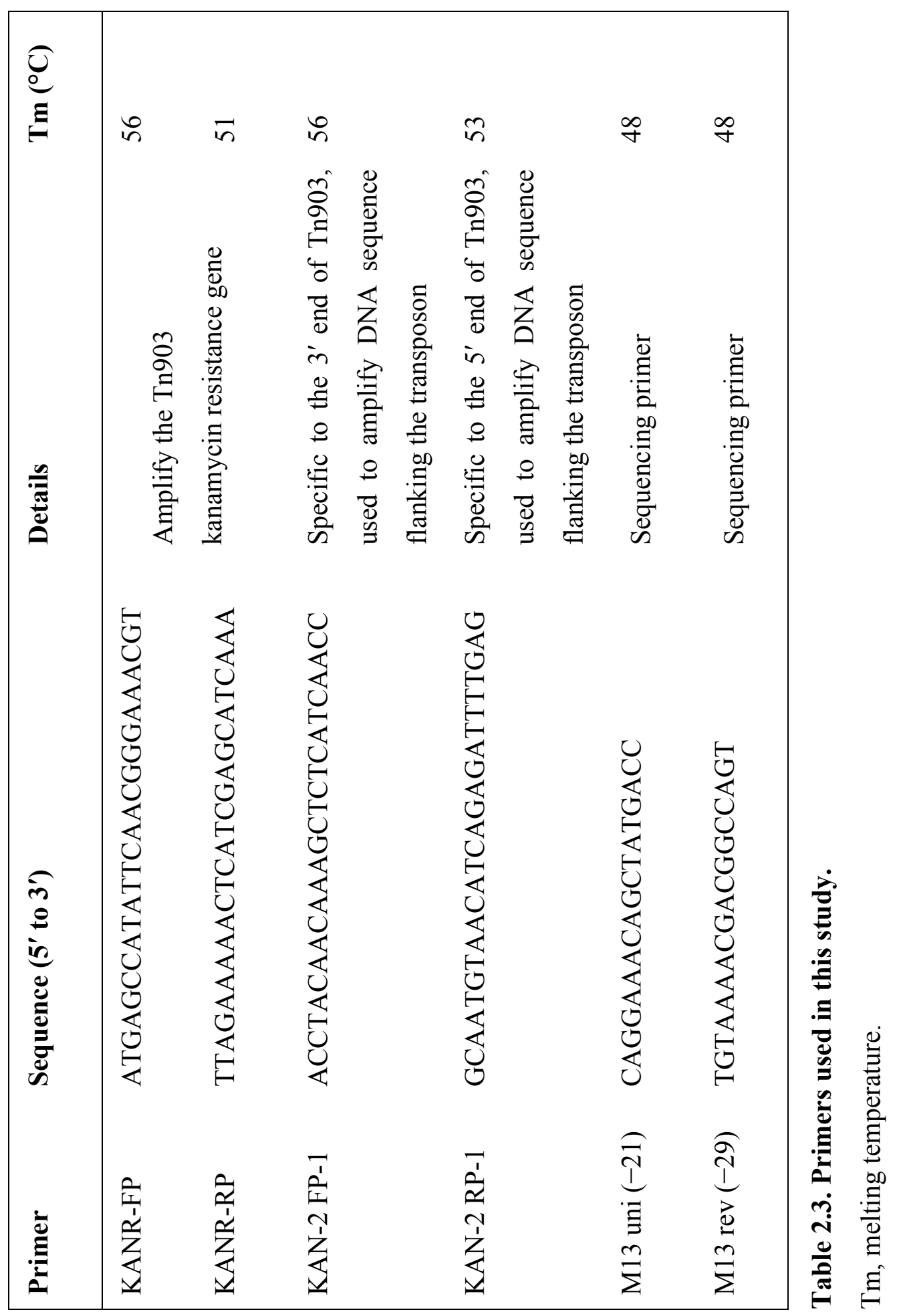




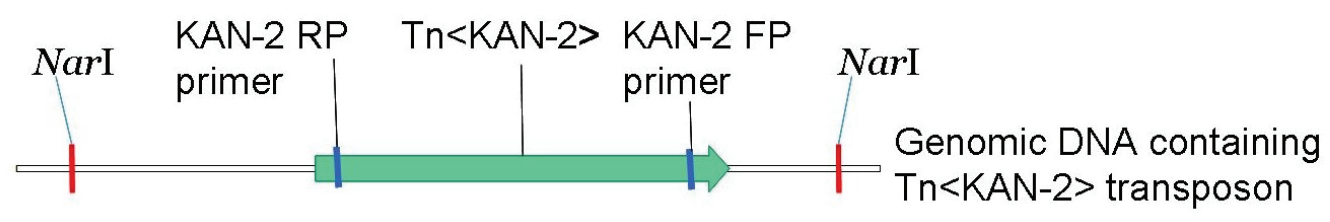

Restriction of genomic DNA with Narl and religation
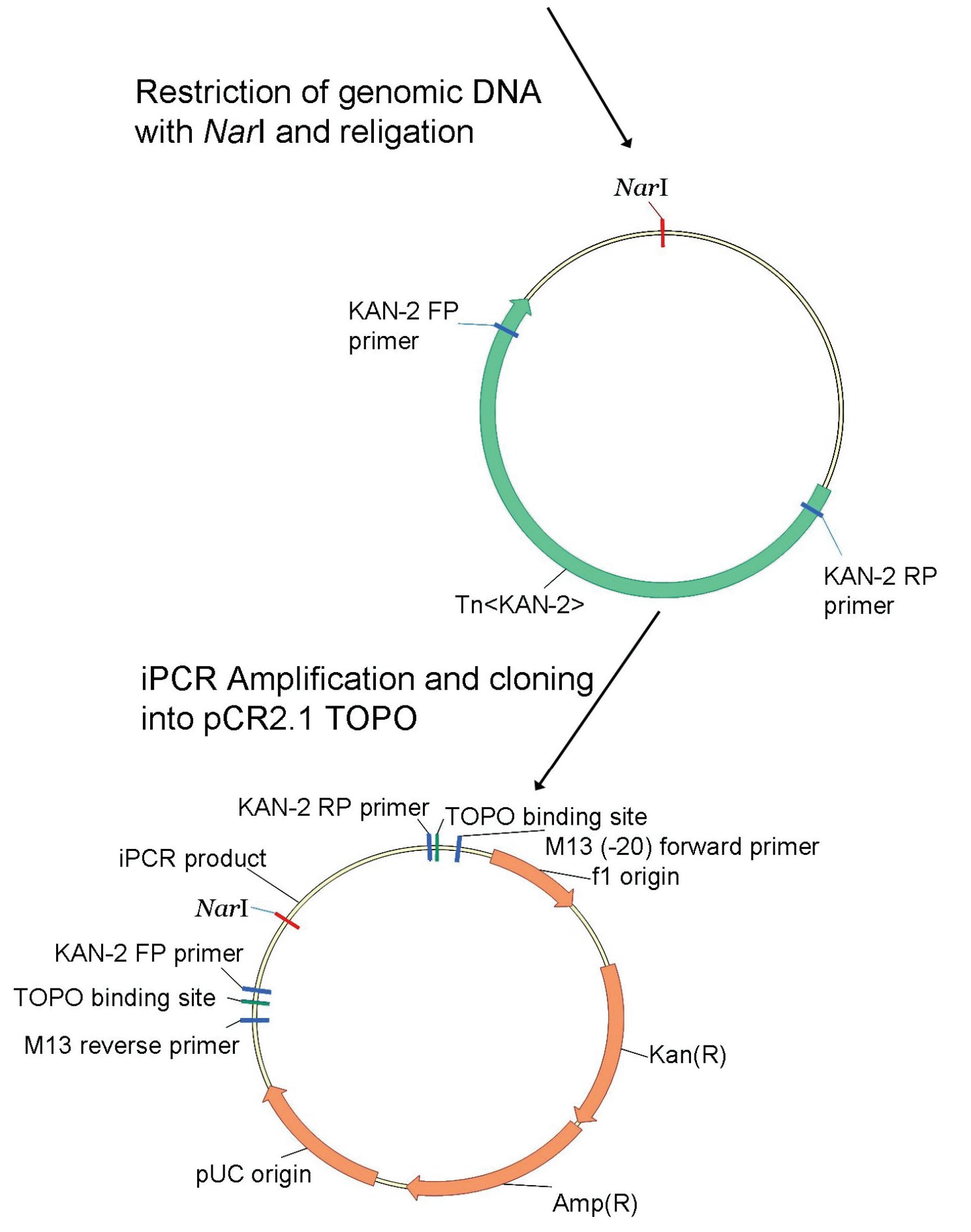

Figure 2.4. Principle of Inverse PCR (iPCR) and cloning. For illustration purposes only the restriction endonuclease NarI is used. 
Approximately $2 \mu \mathrm{g}$ of genomic DNA was digested with a panel of restriction enzymes. The restrictions were performed following the manufacturer's instructions and the efficiency of restriction was checked by agarose gel electrophoresis on $0.8 \%$ agarose gels. The DNA was then separated from the restriction enzymes by phenol/chloroform extraction (Sambrook et al., 1987). Briefly, the DNA was diluted to a volume of $200 \mu 1$ and an equal volume of phenol:chloroform:isoamylalcohol (25:24:1) (Sigma-Aldrich) was added. The contents of the tube were mixed by agitation until an emulsion formed. The mixture was centrifuged at $12,000 \times \mathrm{g}$ for $15 \mathrm{~s}$ at room temperature to separate the organic and aqueous phases. The aqueous phase, containing the DNA, was then transferred to a fresh sterile eppendorf and the DNA precipitated by adding an equal volume of isopropanol (2-propanol). The tube was mixed by agitation and the DNA pelleted by centrifugation at $14,000 \times \mathrm{g}$ for $10 \mathrm{~min}$. The DNA was resuspended in $17 \mu \mathrm{l}$ $\mathrm{dH}_{2} \mathrm{O}$, to which $1 \mu \mathrm{l}$ ligation buffer $(10 \times)$ and $1 \mu \mathrm{l}$ of T4 DNA ligase (Roche Applied Science) were added. After incubation at room temperature overnight, the reaction was inactivated by heating in a $80{ }^{\circ} \mathrm{C}$ waterbath for $20 \mathrm{~min}$. The ligation mixture was then dialysed against $\mathrm{dH}_{2} \mathrm{O}$ on a $0.025 \mu \mathrm{m}$ MF-Millipore membrane (hydrophilic, Millipore) for $1 \mathrm{~h}$ at room temperature prior to iPCR.

The PCR was performed as previously described in Section 2.11 using $1 \mu$ l religated DNA as the template. The amplification cycle consisted of a denaturing period of $5 \mathrm{~min}$ at $96{ }^{\circ} \mathrm{C}$, followed by 30 cycles of $30 \mathrm{~s}$ denaturation at $95^{\circ} \mathrm{C}, 1 \mathrm{~min}$ annealing at $56^{\circ} \mathrm{C}$ and 1 min of elongation at $72{ }^{\circ} \mathrm{C}$. A final elongation period of 5 min at $72{ }^{\circ} \mathrm{C}$ was then followed by cooling down to $4{ }^{\circ} \mathrm{C}$. The PCR products were then analysed by agarose gel electrophoresis. 


\subsection{TA-Cloning}

The iPCR products were cloned using the TOPO TA Cloning Kit (with pCR2.1-TOPO vector) with One Shot TOP10 chemically competent E. coli DH5- $\alpha$ from Invitrogen according to the manufacturer's instructions. The pCR2.1-TOPO vector was used since it has 3'-T overhangs to allow efficient cloning of Taq polymerase-generated fragments ( $T a q$ adds an $\mathrm{A}$ to the $5^{\prime}$ end of the region amplified). The $\mathrm{T}$ overhangs are also covalently linked to topoisomerase I from the vaccinia virus, mediating the ligation of the PCR-generated fragment into the cloning vector.

\subsection{DNA sequencing and bioinformatics analysis}

The concentration of purified plasmid DNA was determined by agarose gel electrophoresis. Ethanol-precipitated DNA, provided to MWG Biotech (London) for sequencing, was prepared as follows. The plasmid DNA (1-2 $\mu \mathrm{g})$ was diluted in a final

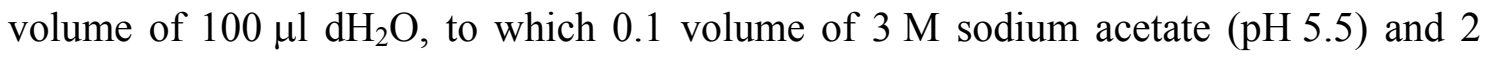
volumes of $100 \%$ ethanol were added. The sample was mixed after $5 \mathrm{~min}$ at $4{ }^{\circ} \mathrm{C}$ and centrifuged at $14,000 \times \mathrm{g}$ for $5 \mathrm{~min}$ at room temperature. The pellet was resuspended in $200 \mu 170 \%$ ethanol and centrifugation was repeated. The supernatant was discarded, and the DNA pellet was air-dried at room temperature. DNA sequencing was performed by MWG Biotech using primers M13 uni (-21) and M13 rev (-29) (see Table 2.3).

All sequence manipulations (i.e. cutting and pasting, generation of complement sequence, identification of restriction and primer binding sites) were done using the programme Vector NTI from Invitrogen (http://www.invitrogen.com). The sequence was then analysed using various tools of the Vector NTI package, such as AlignX, ContigExpress, ORF Finder and the Translation tool. The complete DNA sequences obtained were also searched directly against the non-redundant database at NCBI, using 
either BLASTN for a nucleotide search or BLASTX to search the database with the sequence translated in all six frames. The resulting putative proteins were searched against the database using BLASTP (Altschul et al., 1997; Schaffer et al., 2001) at www.ncbi.nlm.nih.gov. The search was performed on the non-redundant database (nr) limited to the phylum Bacteria. The most significant results were used to assign a function to the putative protein. Further analysis of the DNA sequence was carried out with a neural network promoter prediction method (Reese \& Eeckman, 1995), in an attempt to identify putative promoters. The software NNPP version 2.2 used is available at http://www.fruitfly.org/seq_tools/promoter.html. 


\subsection{Composition of solutions and reagents}

All solutions and media were prepared with deionised water, except where indicated. Bacteriological medium components were obtained from Oxoid. All chemicals, antibiotics and vitamins were purchased from Sigma-Aldrich and were of HPLC grade.

\subsubsection{Growth media and supplements}

All media were prepared and dispensed prior to autoclaving at $121{ }^{\circ} \mathrm{C}$ and 15 pounds per square inch of pressure for $15 \mathrm{~min}$.

Luria-Bertani broth (per litre) (Atlas, 1996):

$\begin{array}{ll}\text { Tryptone } & 10 \mathrm{~g} \\ \text { Sodium chloride }(\mathrm{NaCl}) & 10 \mathrm{~g}(172 \mathrm{mM}) \\ \text { Yeast extract } & 5 \mathrm{~g}\end{array}$

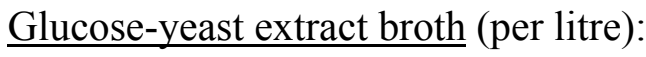

$\begin{array}{ll}\text { Glucose } & 10 \mathrm{~g}(55 \mathrm{mM}) \\ \text { Yeast extract } & 10 \mathrm{~g}\end{array}$

$\underline{\mathrm{CDM} I}$ (per litre):

$\begin{array}{ll}\text { Glucose } & 2 \mathrm{~g}(11 \mathrm{mM}) \\ \text { Ammonium sulphate }\left[\left(\mathrm{NH}_{4}\right)_{2} \mathrm{SO}_{4}\right] & 1 \mathrm{~g}(7.5 \mathrm{mM}) \\ \text { Dipotassium phosphate anhydrous }\left(\mathrm{K}_{2} \mathrm{HPO}_{4}\right) & 1 \mathrm{~g}(5.7 \mathrm{mM}) \\ \text { Salts solution (recipe provided below) } & 10 \mathrm{ml} \\ \text { Trace elements solution (recipe provided below) } & 1 \mathrm{ml}\end{array}$


CDM IIA $1 \%$ Glucose (per litre):

$\begin{array}{ll}\text { Glucose } & 10 \mathrm{~g}(55 \mathrm{mM}) \\ \text { Ammonium sulphate }\left(\left(\mathrm{NH}_{4}\right)_{2} \mathrm{SO}_{4}\right) & 1 \mathrm{~g}(7.5 \mathrm{mM}) \\ \text { Dipotassium phosphate anhydrous }\left(\mathrm{K}_{2} \mathrm{HPO}_{4}\right) & 2.75 \mathrm{~g}(15.8 \mathrm{mM}) \\ \text { Potassium phosphate }\left(\mathrm{KH}_{2} \mathrm{PO}_{4}\right) & 2.25 \mathrm{~g}(16.5 \mathrm{mM}) \\ \text { Salts solution } & 10 \mathrm{ml} \\ \text { Trace elements solution } & 1 \mathrm{ml}\end{array}$

For CDM IIA $0.1 \%$ Glucose, , only $1 \mathrm{~g}$ of glucose was used per litre (final concentration $5.5 \mathrm{mM})$.

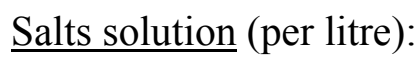

$\begin{array}{ll}\text { Magnesium sulphate }\left(\mathrm{MgSO}_{4} .7 \mathrm{H}_{2} \mathrm{O}\right) & 25 \mathrm{~g}(0.1 \mathrm{M}) \\ \text { Ferrous sulphate }\left(\mathrm{FeSO}_{4} .7 \mathrm{H}_{2} \mathrm{O}\right) & 0.28 \mathrm{~g}(1 \mathrm{mM}) \\ \text { Manganous sulphate }\left(\mathrm{MnSO}_{4} .4 \mathrm{H}_{2} \mathrm{O}\right) & 1.7 \mathrm{~g}(7.6 \mathrm{mM}) \\ \text { Sodium chloride }(\mathrm{NaCl}) & 0.6 \mathrm{~g}(10 \mathrm{mM}) \\ \text { Sodium molybdate }\left(\mathrm{Na}_{2} \mathrm{MoO}_{4} .2 \mathrm{H}_{2} \mathrm{O}\right) & 0.1 \mathrm{~g}(0.4 \mathrm{mM}) \\ \text { Zinc sulphate }\left(\mathrm{ZnSO} \mathrm{O}_{4} .7 \mathrm{H}_{2} \mathrm{O}\right) & 0.06 \mathrm{~g}(0.2 \mathrm{mM}) \\ \text { Calcium chloride }\left(\mathrm{CaCl}_{2} .2 \mathrm{H}_{2} \mathrm{O}\right) & 0.01 \mathrm{~g}(0.07 \mathrm{mM}) \\ \text { Hydrochloric acid, } 0.1 \mathrm{M} & 1 \text { litre }\end{array}$


Trace elements solution (per litre):

$\begin{array}{lc}\text { Hydrochloric acid }(25 \%) & 6.5 \mathrm{ml} \\ \text { Ferrous chloride }\left(\mathrm{FeCl}_{2} .4 \mathrm{H}_{2} \mathrm{O}\right) & 1.5 \mathrm{~g}(7.5 \mathrm{mM}) \\ \text { Borate }\left(\mathrm{H}_{3} \mathrm{BO}_{3}\right) & 60 \mathrm{mg}(1 \mathrm{mM}) \\ \text { Manganous chloride }\left(\mathrm{MnCl}_{2} .4 \mathrm{H}_{2} \mathrm{O}\right) & 100 \mathrm{mg}(0.5 \mathrm{mM}) \\ \text { Cobalt chloride }\left(\mathrm{CoCl}_{2} \cdot 6 \mathrm{H}_{2} \mathrm{O}\right) & 120 \mathrm{mg}(0.5 \mathrm{mM}) \\ \text { Zinc chloride }\left(\mathrm{ZnCl}_{2}\right) & 70 \mathrm{mg}(0.5 \mathrm{mM}) \\ \text { Nickel chloride }\left(\mathrm{NiCl}_{2} .6 \mathrm{H}_{2} \mathrm{O}\right) & 25 \mathrm{mg}(0.1 \mathrm{mM}) \\ \text { Copper chloride }\left(\mathrm{CuCl}_{2} .2 \mathrm{H}_{2} \mathrm{O}\right) & 15 \mathrm{mg}(0.1 \mathrm{mM}) \\ \text { Sodium molybdate }\left(\mathrm{Na}_{2} \mathrm{MoO}_{4} \cdot 2 \mathrm{H}_{2} \mathrm{O}\right) & 25 \mathrm{mg}(0.1 \mathrm{mM})\end{array}$

The salts and the trace elements solutions were distributed in $200 \mathrm{ml}$ bottles, autoclaved and stored at $4{ }^{\circ} \mathrm{C}$. 


\subsubsection{Composition of media supplements}

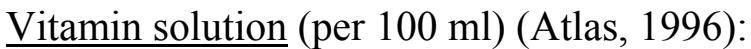

$\begin{array}{lc}\text { Biotin } & 4 \mathrm{mg}(160 \mu \mathrm{M}) \\ p \text {-aminobenzoic acid } & 10 \mathrm{mg}(260 \mu \mathrm{M}) \\ \text { Folic acid } & 4 \mathrm{mg}(90 \mu \mathrm{M}) \\ \text { Calcium pantothenate } & 10 \mathrm{mg}(420 \mu \mathrm{M}) \\ \text { Nicotinic acid } & 10 \mathrm{mg}(813 \mu \mathrm{M}) \\ \text { Vitamin B12 } & 0.2 \mathrm{mg}(1 \mu \mathrm{M}) \\ \text { Thiamine hydrochloride } & 10 \mathrm{mg}(296 \mu \mathrm{M}) \\ \text { Pyridoxine (B6) hydrochloride } & 20 \mathrm{mg}(975 \mu \mathrm{M}) \\ \text { Thioctic acid } & 10 \mathrm{mg}(485 \mu \mathrm{M}) \\ \text { Riboflavin } & 1 \mathrm{mg}(26 \mu \mathrm{M})\end{array}$

The vitamin solution was filter-sterilised to avoid heat degradation, aliquoted in $1.5 \mathrm{ml}$ eppendorfs and stored at $-20^{\circ} \mathrm{C}$.

Thiamine solution:

$\begin{array}{ll}\text { Thiamine hydrochloride } & 5 \mathrm{mg}(0.7 \mathrm{mM}) \\ \text { Distilled water } & 20 \mathrm{ml}\end{array}$

The thiamine solution was filter-sterilised to avoid heat degradation, aliquoted in $1.5 \mathrm{ml}$ eppendorfs and stored at $-20^{\circ} \mathrm{C}$. 


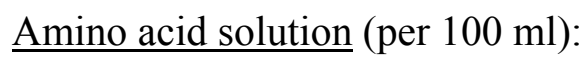

\begin{tabular}{|c|c|}
\hline L-Gln & $292 \mathrm{mg}(20 \mathrm{mM})$ \\
\hline L-Arg & $126.1 \mathrm{mg}(7 \mathrm{mM})$ \\
\hline L-Ile & $52 \mathrm{mg}(4 \mathrm{mM})$ \\
\hline L-Leu & $52 \mathrm{mg}(4 \mathrm{mM})$ \\
\hline L-Tyr, diNa & $52 \mathrm{mg}(2.8 \mathrm{mM})$ \\
\hline L-Val & $46 \mathrm{mg}(3.9 \mathrm{mM})$ \\
\hline L-His.HCl & $42 \mathrm{mg}(2.7 \mathrm{mM})$ \\
\hline L-Phe & $32 \mathrm{mg}(1.9 \mathrm{mM})$ \\
\hline L-Met & $31 \mathrm{mg}(2 \mathrm{mM})$ \\
\hline L-Glu & $15 \mathrm{mg}(1 \mathrm{mM})$ \\
\hline L-Asp & $13.3 \mathrm{mg}(0.7 \mathrm{mM})$ \\
\hline $\mathrm{L}-\mathrm{Asn} . \mathrm{H}_{2} \mathrm{O}$ & $13.2 \mathrm{mg}(1 \mathrm{mM})$ \\
\hline L-Pro & $11.5 \mathrm{mg}(1 \mathrm{mM})$ \\
\hline L-Ser & $10.5 \mathrm{mg}(1 \mathrm{mM})$ \\
\hline L-Trp & $10 \mathrm{mg}(0.5 \mathrm{mM})$ \\
\hline DL-Ala & $8.9 \mathrm{mg}(1 \mathrm{mM})$ \\
\hline Gly & $7.5 \mathrm{mg}(1 \mathrm{mM})$ \\
\hline Distilled water & $100 \mathrm{ml}$ \\
\hline
\end{tabular}

The amino acid solution was filter-sterilised and stored at $4{ }^{\circ} \mathrm{C}$. 
Pyrimidine solution (per $100 \mathrm{ml}$ ):
Uracil
$20 \mathrm{mg}(1.8 \mathrm{mM})$
Thymine
$20 \mathrm{mg}(1.6 \mathrm{mM})$
Cytosine
$20 \mathrm{mg}(1.8 \mathrm{mM})$

The pyrimidine solution was gently heated to dissolve all components. It was then filter-sterilised and stored at $4{ }^{\circ} \mathrm{C}$.

\section{$\underline{\text { Antibiotics: }}$}

Stock solutions of $100 \mathrm{mg} / \mathrm{ml}$ kanamycin and ampicillin were prepared in distilled water, filter-sterilised and stocked at $-20{ }^{\circ} \mathrm{C}$. Stock solution of $100 \mathrm{mg} / \mathrm{ml}$ chloramphenicol was prepared in $100 \%$ ethanol and stocked at $-20{ }^{\circ} \mathrm{C}$. 


\subsubsection{Reagents}

Tris-EDTA (TE) buffer $(1 \times)$ : 10 mM Tris-HCl, 1 mM EDTA pH 8.0.

Tris-acetate-EDTA buffer (TAE) (50×):

$\begin{array}{ll}\text { Tris- } \mathrm{HCl} & 240 \mathrm{~g}(2 \mathrm{M}) \\ \text { Glacial acetic acid } & 57.1 \mathrm{ml} \\ \text { EDTA } 0.5 \mathrm{M} & 100 \mathrm{ml}(50 \mathrm{mM}) \\ \mathrm{dH}_{2} \mathrm{O} & \text { to } 1000 \mathrm{ml}\end{array}$

The $\mathrm{pH}$ was then adjusted to 8.5 . The solution was diluted $50 \times$ to prepare agarose gels and used as running buffer.

\begin{tabular}{|c|c|c|}
\hline SOC broth: & Magnesium chloride $\left(\mathrm{MgCl}_{2}\right)$ & $10 \mathrm{mM}$ \\
\hline & Magnesium sulphate $\left(\mathrm{MgSO}_{4}\right)$ & $10 \mathrm{mM}$ \\
\hline & Potassium chloride $(\mathrm{KCl})$ & $2.5 \mathrm{mM}$ \\
\hline & Sodium chloride $(\mathrm{NaCl})$ & $10 \mathrm{mM}$ \\
\hline & Tryptone & $20 \mathrm{~g} / 1$ \\
\hline & Yeast extract & $5 \mathrm{~g} / 1$ \\
\hline & Glucose & $20 \mathrm{mM}$ \\
\hline
\end{tabular}

SOC broth was dispensed in universals and autoclaved at $121^{\circ} \mathrm{C}$ and 15 pounds per inch of pressure for $15 \mathrm{~min}$. 


\section{Chapter III \\ Physiological Response of Rhodococcus erythropolis SQ1 to Starvation}


Members of the genus Rhodococcus are widely distributed and have been isolated from diverse environments, as described in Chapter I. Their ability to survive in low-energy and nutrient environments is significant due to the role they play in bioremediation (e.g. in the case of Rhodococcus erythropolis and Rhodococcus ruber) and pathogenesis (e.g. in the case of Rhodococcus equi and Rhodococcus fascians). From an industrial perspective, it has been shown that nitrogen starvation may elicit biosurfactant production in $R$. ruber (Philp et al., 2002) and R. erythropolis (Kim et al., 1990). Nitrogen starvation also decreased the hydrophobicity of Rhodococcus corallinus (Sanin et al., 2003), with consequences for bioremediation and microbial deemulsification (Van Hamme et al., 2003). Carbon and/or energy starvation can also be important factors for other chemical/biosynthesis processes. For instance, ultramicrobacteria (UMB) are produced by carbon/energy starvation, and used for microbial-enhanced oil recovery (Van Hamme et al., 2003).

There has been little study to date of the processes by which rhodococci survive starvation, or indeed of the effects of starvation on rhodococci. It has been shown however that $R$. corallinus can grow after incubation for up to 5 months in carbon- or nitrogen-free medium (Sanin, 2003). The objective of the study reported in this chapter was to characterise the physiological response of Rhodococcus to starvation (in this study, carbon was chosen as the limiting nutrient) and to investigate whether carbon starvation can induce cross-protection in Rhodococcus to a variety of environmental stresses, including heat and oxidative stress. 


\subsection{Selection of an appropriate Rhodococcus strain for the study of carbon starvation}

There are many parameters that influenced the choice of the particular strain of Rhodococcus for this study. In order to investigate the physiological response of Rhodococcus to starvation (the focus of this chapter), it was essential to be able to accurately limit the nutrient or nutrients for which the bacterium was being starved and to be able to monitor the growth of Rhodococcus on this medium. The growth behaviour of Rhodococcus on chemically defined medium was therefore considered. The second part of this study (Chapter IV) however is concerned with the generation of a mutant bank and the screening of that mutant bank for starvation or stationary phase survival mutants. Therefore additional criteria were applied to the selection of the appropriate strain. These included sensitivity to antibiotics that may be used as selective pressure to allow for screening of mutants and transformants, and the transformability of Rhodococcus with plasmid DNA

\subsubsection{Design of a chemically defined medium suitable for studying the effect of carbon starvation on Rhodococcus}

Thirteen strains representative of four taxa $-R$. erythropolis, $R$. ruber, $R$. equi and R. fascians - were selected. These strains are described in Table 2.1. Of the forty-two species of Rhodococcus identified to date (Euzéby, 2007), these four species were selected initially for their industrial, environmental and/or medical significance. All thirteen strains selected were able to grow readily on Luria-Bertani (LB) or glucose yeast extract (GYE) media, whether prepared as broth or agar plates. The basic chemically defined medium described by Goodhue et al. (1986) was selected as the basis for designing a chemically defined medium appropriate for Rhodococcus. It 
should be noted that the basic medium described by Goodhue et al. (1986) does not include vitamins, although these are widely reported to be necessary for the growth of some rhodococci (Goodfellow \& Alderson, 1977; Goodfellow, 1989). Therefore, in the first instance, in this study supplementation with either a complex mix of vitamins (described in Chapter II) or with $0.7 \mathrm{mM}$ thiamine was performed in order to ensure growth of all strains and to avoid complex deficiencies. These modified media were labelled as CDM IA and CDM IB, respectively. Glucose $(0.2 \% \mathrm{w} / \mathrm{v})$ was chosen as the carbon and energy source (Goodhue et al., 1986). Growth was assessed visually after $48 \mathrm{~h}$ of incubation at $27^{\circ} \mathrm{C}$ and agitation at $200 \mathrm{rpm}$. The results are shown in Table 3.1 .

Strains of $R$. ruber Ac72, Ac74, Ac82 and Ac87 grown in CDM IA and IB broth clumped extensively. A significant amount of clumping was also observed for R. erythropolis Ac201. Extensive clumping of bacterial cells is undesirable since this leads to inaccurate cell counts, whether evaluated by determining viable plate counts or optical density. Although dense cultures were obtained for most strains tested in $\mathrm{CDM}$ IA and IB, it is possible that the low concentrations of carbon $(0.2 \% \mathrm{w} / \mathrm{v}$ glucose, i.e. $11 \mathrm{mM})$ and phosphate $\left(6 \mathrm{mM} \mathrm{K}_{2} \mathrm{HPO}_{4}\right)$ used may be growth-limiting. Furthermore, although there was no evidence of $\mathrm{pH}$ fluctuation, the low concentrations of phosphate and the limited buffering capacity provided may lead to large variations in $\mathrm{pH}$ during lengthy periods of incubation. Therefore, the glucose concentration was increased from 0.2 to $1 \% \mathrm{w} / \mathrm{v}$. The phosphate concentration was also increased to $32.3 \mathrm{mM}$ using $15.8 \mathrm{mM}$ dipotassium hydrogen phosphate $\left(\mathrm{K}_{2} \mathrm{HPO}_{4}\right)$ and $16.5 \mathrm{mM}$ potassium dihydrogen phosphate $\left(\mathrm{KH}_{2} \mathrm{PO}_{4}\right)$, thereby buffering the medium at $\mathrm{pH} 6.2-7.4$ (Sambrook et al., 1987). Good growth was attained in this medium (CDM IIA; see Table 3.1) for all species and strains tested, except for $R$. equi. Supplementation with 
amino acids and pyrimidines (see Chapter II for composition) was required for good growth of R. equi (CDM IIB; Table 3.1). 


\begin{tabular}{|c|c|c|c|c|c|}
\hline \multirow{2}{*}{ Species } & \multirow{2}{*}{ Strain } & \multicolumn{4}{|c|}{ CDM } \\
\hline & & IA & IB & IIA & IIB \\
\hline \multirow[t]{4}{*}{ R. erythropolis } & SQ1 & +++ & +++ & ++++ & $\mathrm{ND}$ \\
\hline & Ac201 & +++ & +++ & ++++ & $\mathrm{ND}$ \\
\hline & NCIMB 8147 & +++ & +++ & ++++ & ND \\
\hline & NCIMB 8863 & +++ & +++ & ++++ & $\mathrm{ND}$ \\
\hline \multirow[t]{4}{*}{ R. equi } & GV1 & + & + & ++ & ++++ \\
\hline & VI 1 & + & - & + & ++++ \\
\hline & NCIMB 10027 & - & + & + & ++++ \\
\hline & NCIMB 10701 & + & + & + & ++++ \\
\hline \multirow[t]{4}{*}{ R. ruber } & Ac72 & ++ & ++ & +++ & $\mathrm{ND}$ \\
\hline & Ac74 & ++ & ++ & +++ & $\mathrm{ND}$ \\
\hline & Ac82 & +++ & +++ & ++++ & $\mathrm{ND}$ \\
\hline & Ac87 & ++ & ++ & +++ & ND \\
\hline R. fascians & Ac170 & +++ & +++ & ++++ & ND \\
\hline
\end{tabular}

Table 3.1. Growth of Rhodococcus species in a range of chemically defined media.

-, no growth; + to ++++ , increasing growth; ND, not determined.

CDM IA and CDM IB correspond to a basic chemically defined medium containing $0.2 \%$ glucose described by Goodhue et al. (1986) supplemented with either $1 \% \mathrm{v} / \mathrm{v}$ of vitamin mix (described in Chapter II) or $0.7 \mathrm{mM}$ thiamine, respectively.

CDM IIA corresponds to CDM IA with $1 \%$ glucose and is phosphate-buffered.

CDM IIB corresponds to CDM IIA supplemented with amino acids and pyrimidines. 


\subsubsection{Antibiotic resistance markers for screening of transformants and mutants of Rhodococcus}

Antibiotic resistance is an essential tool for genetic manipulation of microorganisms. Most resistance markers are encoded by a single gene, thus making them easily transferable from one organism to another. The majority of cloning vectors are designed with specific antibiotic resistance markers, allowing the isolation of the organisms harbouring the vector. Transposons, frequently used for random mutagenesis, generally possess an antibiotic resistance gene. It is therefore essential that the Rhodococcus strain selected for this study is sensitive to antibiotics that may be used in a later stage of the project. The cloning vectors used in this study carry resistance genes for ampicillin, kanamycin and chloramphenicol, and the transposon system we proposed to use harbours a kanamycin resistance gene. It was therefore essential to verify the antibiotic sensitivity profile of the available rhodococcal strains.

All the strains considered for this study were plated on GYE agar containing either $100 \mu \mathrm{g} \mathrm{ml}^{-1}$ of ampicillin, $200 \mu \mathrm{g} \mathrm{ml}^{-1}$ of kanamycin or $40 \mu \mathrm{g} \mathrm{ml}^{-1}$ of chloramphenicol. The genes coding for resistance to these antibiotics are used as the selective markers on the cloning vectors used to date in Rhodococcus (Dabbs, 1998; Quan \& Dabbs, 1993; Veselý et al., 2003). Antibiotic concentrations were chosen according to the literature regarding the use of these vectors in Rhodococcus (Quan \& Dabbs, 1993; Veselý et al., 2003).

Except for the phytopathogen $R$. fascians, all the strains tested were sensitive to ampicillin, kanamycin and chloramphenicol (Table 3.2). R. fascians Ac170 was resistant to all three antibiotics. Based on these observations, the Rhodococcus-E. coli 
shuttle vectors and the transposon mutagenesis system used later in this study are unsuitable for R. fascians Ac170. 


\begin{tabular}{|c|c|c|c|c|}
\hline \multirow[b]{2}{*}{ Strain } & \multicolumn{4}{|c|}{ Growth medium } \\
\hline & GYE & $\begin{array}{c}\text { GYE + } \\
\text { ampicillin }\end{array}$ & $\begin{array}{c}\text { GYE + } \\
\text { kanamycin }\end{array}$ & $\begin{array}{c}\text { GYE + } \\
\text { chloramphenicol }\end{array}$ \\
\hline $\begin{array}{l}\text { R. fascians } \\
\text { Ac170 }\end{array}$ & + & + & + & + \\
\hline $\begin{array}{l}\text { R. erythropolis } \\
\text { Ac201 }\end{array}$ & + & - & - & - \\
\hline $\begin{array}{l}\text { R. erythropolis } \\
\text { NCIMB } 8147\end{array}$ & + & - & - & - \\
\hline $\begin{array}{l}\text { R. erythropolis } \\
\text { NCIMB } 8863\end{array}$ & + & - & - & - \\
\hline $\begin{array}{l}\text { R. erythropolis } \\
\text { SQ1 }\end{array}$ & + & - & - & - \\
\hline $\begin{array}{l}\text { R. ruber } \\
\text { Ac72 }\end{array}$ & + & - & - & - \\
\hline $\begin{array}{l}\text { R. ruber } \\
\text { Ac74 }\end{array}$ & + & - & - & - \\
\hline $\begin{array}{l}\text { R. ruber } \\
\text { Ac82 }\end{array}$ & + & - & - & - \\
\hline $\begin{array}{l}\text { R. ruber } \\
\text { Ac87 }\end{array}$ & + & - & - & - \\
\hline
\end{tabular}

Table 3.2. Growth of selected Rhodococcus strains on GYE agar containing antibiotics.

Concentrations of $100 \mu \mathrm{g} \mathrm{ml}^{-1}$ ampicillin, $200 \mu \mathrm{g} \mathrm{ml}^{-1}$ kanamycin and $40 \mu \mathrm{g} \mathrm{ml}^{-1}$ chloramphenicol were used. Cultures were incubated at $27^{\circ} \mathrm{C}$ until colonies could be clearly observed, or for up to 4 days.

+ , growth; -, no growth. 


\subsubsection{Transformability of rhodococcal strains}

To date, there are only a limited number of publications reporting the genetic manipulation of Rhodococcus sp. There are a few reports concerning the introduction of DNA to Rhodococcus species using conjugation. Conjugation has been reported for $R$. erythropolis BD2 with plasmid pBD2 (Dabrock et al., 1994), R. fascians with plasmid pD188 (Desomer et al., 1988) and R. erythropolis SQ1 with plasmids pB264 and pAN12 derivatives (Lessard et al., 2004; Yang et al., 2007b). These conjugative plasmids can however be so large as to be unwieldy for molecular manipulation. pBD2 is approximately $210 \mathrm{~kb}$, while pD188 is $138 \mathrm{~kb}$ (Dabrock et al., 1994; Desomer et al., 1988). The transfer efficiency of pB264 $(5 \mathrm{~kb})$ is low at $7.3 \times 10^{-7}$ per recipient (Lessard et al., 2004). A recent study with pAN12 derivatives has yielded promising results with conjugation efficiencies up to $7 \times 10^{-4}$ tranconjugants per recipient cell (Yang et al., 2007a).

$R$. erythropolis, $R$. ruber, $R$. equi and $R$. fascians have all been transformed by electroporation (Chauvaux et al., 2001; Desomer et al., 1990; Sekizaki et al., 1998; Treadway et al., 1999). Several shuttle vectors have been designed that allow transfer of genes to and from rhodococci by electroporation, such as pMVS301 that replicates in $E$. coli, R. equi, R. erythropolis and R. globerulus (Singer \& Finnerty, 1988); pDA21 that replicates in E. coli, R. equi, R. erythropolis and R. rhodochrous (Dabbs, 1998), pRE-7 that replicates in E. coli, R. ruber and R. equi (Chauvaux et al., 2001; Zheng et al., 1997) and pSRK21 that replicates in E. coli and R. erythropolis (Veselý et al., 2003). In addition, random transposon mutagenesis has been achieved in $R$. erythropolis and R. equi using electroporation (Fernandes et al., 2001; Mangan \& Meijer, 2001). It was therefore decided to investigate the efficiency of transformation of selected 
Rhodococcus strains by electroporation, with the aim of using this technique to generate a mutant bank (Chapter IV).

Three strains, $R$. equi NCIMB 10027, R. equi NCIMB 10701 and R. erythropolis SQ1, were tested for their transformability (i.e. the ease with which transformants can be obtained in the laboratory). Two protocols were used to produce electrocompetent cells. The main differences between these protocols is that cells are grown in LB alone in one (Denome et al., 1993), whereas in the other LB is supplemented with sucrose, glycine and isoniazid (Zheng et al., 1997). Glycine is often used in the growth medium to prepare competent cells as it disrupts the structure of the peptidoglycan. Isoniazid is an antibiotic that disrupts the synthesis of mycolic acids, a component of the cell wall of actinomycetes. The transformation efficiencies were determined using plasmids pSRK21 and pRE-7.

pSRK2 1 is a small $(5.8 \mathrm{~kb})$ shuttle vector based on Corynebacterium vectors and was designed specifically for transfer between E. coli and R. erythropolis (Veselý et al., 2003). It is based on the fusion of pSR1, a Corynebacterium glutamicum plasmid (Archer \& Sinskey, 1993), and pK19, an E. coli shuttle vector bearing both an ori (origin of replication) and a kanamycin resistance gene (Pridmore, 1987). A maximum transformation efficiency of $7 \times 10^{4}$ transformants $\mu \mathrm{g}^{-1}$ DNA was achieved with pSRK21 in R. erythropolis CCM2595 (Veselý et al., 2003).

pRE-7 is similar in size $(5.9 \mathrm{~kb})$ to pSRK21 and was designed as a shuttle vector between E. coli and R. equi (Zheng et al., 1997). It was created by combining the ori of R. equi strain 103 virulence-associated plasmid pOTS, the kanamycin resistance gene of plasmid pACYC177 and the plasmid pBluescript $\mathrm{KS}(+)$ (ColE1 ori) (Zheng et al., 
1997). A maximum transformation efficiency of $2.5 \times 10^{7}$ transformants $\mu \mathrm{g}^{-1}$ DNA was achieved with pRE-7 in R. equi $103^{-}$(pOTS-cured strain).

R. equi NCIMB 10027 and R. equi NCIMB 10701 competent cells were initially prepared using the method described by Zheng et al. (1997). With plasmid pSRK21, transformation frequencies of $9.5 \times 10^{5}$ and $4.45 \times 10^{6}$ transformants $\mu \mathrm{g}^{-1} \mathrm{DNA}$ were obtained for NCIMB 10027 and NCIMB 10701, respectively. R. equi NCIMB 10027 was also transformed with plasmid pRE-7, achieving a frequency of $1 \times 10^{5}$ transformants $\mu \mathrm{g}^{-1}$ DNA.

For R. erythropolis SQ1, frequencies of up to $1.85 \times 10^{5}$ transformants $\mu \mathrm{g}^{-1} \mathrm{pRE}-7$ DNA were achieved using the protocol of Denome et al. (1993). Using the method of Zheng et al., (1997), low cell densities were consistently obtained in the growth medium described. Therefore, a modified medium was used in which isoniazid was omitted, leading to frequencies of up to $1.95 \times 10^{4}$ transformants $\mu \mathrm{g}^{-1} \mathrm{pSRK} 21$ DNA.

The transformation frequencies obtained were comparable to the ones reported by the researchers cited above, i.e. $10^{4}-10^{5}$ transformants $\mu \mathrm{g}^{-1}$ DNA for $R$. erythropolis SQ1 vs $7 \times 10^{4}$ transformants $\mu \mathrm{g}^{-1}$ DNA for $R$. erythropolis CCM2595 (Veselý et al., 2003). Furthermore, these numbers are close to the transformation efficiency recommended by the manufacturer of the transposon system we proposed to use in subsequent stages of the project for random mutagenesis of Rhodococcus $\left(10^{5}\right.$ transformants $\mu \mathrm{g}^{-1} \mathrm{DNA}$; Epicentre). 


\subsubsection{Selection of the study strain}

Representatives of four taxa within the genus Rhodococcus were selected in the initial stages of this study on the basis of their industrial, environmental and/or medical significance. $R$. erythropolis and $R$. ruber are of particular interest due to their biodegradation and biocatalysis capacities, and $R$. fascians and $R$. equi are important pathogens (as reported in Chapter I).

The outcome of growth experiments performed on chemically defined media allowed for a preliminary selection of potential study strains to be made. As reported above (Section 3.1.1), supplementation of chemically defined medium with amino acids and pyrimidines was required for growth of $R$. equi. Since amino acids contain carbon, phosphorus, nitrogen and, in some cases, sulphur, these requirements complicate the estimation of the final quantities of each element in the growth medium. In addition, several strains of $R$. equi can use amino acids as their sole source of carbon, nitrogen, phosphorus and energy (Bizet et al., 1997). This renders determination of the limiting growth factor rather complex. Despite shared mechanisms, it is also well documented that starvation survival mechanisms for carbon/energy and amino acids do differ (e.g. the stringent response in the case of starvation for amino acids) (Autret et al., 1997) (see Chapter I). Therefore, if amino acids were to be used in the growth medium, their abundance or depletion could influence the mechanisms that trigger the starvation survival response. On this basis it was therefore decided to disregard $R$. equi as the study organism, due to its complex nutrient requirements.

R. ruber cells are known to be particularly hydrophobic (Philp et al., 2002). This hydrophobicity is responsible for the extensive clumping observed for all $R$. ruber strains in all three growth media tested in Section 3.1.1. This aggregation would lead to 
difficulties in accurately assessing bacterial growth and physiological status. The reliability of viable plate counts $(\mathrm{CFU} / \mathrm{ml})$ and optical density measurements, for instance, would be questionable.

R. fascians Ac170 grew well on chemically defined medium and no cell aggregation was observed. However, this strain was resistant to all three antibiotics tested in this study (Section 3.1.2). Of particular relevance was the resistance to kanamycin, since the kanamycin resistance marker is present on the cloning vectors and the transposon mutagenesis system at our disposal for this study.

$R$. erythropolis grew well on all the chemically defined media investigated in this study and, with the exception of $R$. erythropolis Ac201, no cell aggregates were observed (Section 3.1.1). It should be noted that $R$. erythropolis SQ1 (one of the strains tested) is a mutant of ATCC 4277-1 (Quan \& Dabbs, 1993), which in turn is an arsenic- and chloramphenicol-sensitive variant of the type strain ATCC 4277 (Dabbs et al., 1990). It is described as having increased transformability compared to the type strain (Quan \& Dabbs, 1993), and has also been used in a targeted gene disruption experiment (van der Geize et al., 2001). Indeed $R$. erythropolis SQ1 stands out for its ease of manipulation in laboratory conditions and its high transformability, achieving frequencies of $>10^{5}$ transformants $\mu \mathrm{g}^{-1}$ plasmid DNA (Section 3.1.3).

On the basis of the analyses above, $R$. erythropolis SQ1 was selected as the study organism for the remainder of this project. 


\subsection{Response of $R$. erythropolis $S Q 1$ to starvation}

It is generally accepted that the environment is oligotrophic (Morita, 1993), sea water and certain types of soil are especially so (Di Mattia et al., 2002; Kjelleberg et al., 1993). Since most bioavailable energy sources are carbon-based, it is justified to study the response of Rhodococcus to carbon starvation. Furthermore, the entrance into stationary phase is of particular interest, since this is when most of the stress-resistance capacities are acquired (see for example Matin, 1991; Smeulders et al., 1999; van Overbeek et al., 1995).

Growth curves are indispensable to the study of the behaviour of bacteria. It was especially important for this project that the growth profile of Rhodococcus in nonlimiting and in limiting environments was established. To this effect, growth was monitored in LB, a complex medium, and in CDM IIA containing 1 and $0.1 \%$ glucose. Survival of $R$. erythropolis SQ1 in the complete absence of nutrients (distilled water) was also monitored.

\subsubsection{Response of $R$. erythropolis $S Q 1$ to growth in complex medium and to multiple-nutrient starvation}

The aim of this experiment was to compare the behaviour of $R$. erythropolis SQ1 in a commonly used rich medium (LB) and when subjected to multiple-nutrient starvation. Growth curves were performed by growing $R$. erythropolis SQ1 in complex (LB) and nutrient-free media (distilled water). In both cases exponential-phase cultures (corresponding to a $24 \mathrm{~h}$ culture) were used to inoculate flasks containing $100 \mathrm{ml}$ of media to a starting cell density of $0.01 \mathrm{OD}_{600 \mathrm{~nm}}$. Cultures were then incubated at $27^{\circ} \mathrm{C}$, at $200 \mathrm{rpm}$. Samples were taken at regular intervals over a period of 43 days. For each 
sample, viable plate counts $(\mathrm{CFU} / \mathrm{ml})$ and optical density $\left(\mathrm{OD}_{600 \mathrm{~nm}}\right)$ were determined. R. erythropolis SQ1 was shown to survive long-term starvation in both $\mathrm{LB}$ and $\mathrm{dH}_{2} \mathrm{O}$ (Figure 3.1). High viable plate counts of up to $4 \times 10^{9} \mathrm{CFU} / \mathrm{ml}$ were observed in LB after 2 days. This figure then decreased to approximately $5 \times 10^{7} \mathrm{CFU} / \mathrm{ml}$ after 30 days in stationary phase. In contrast, the cultures in distilled water did not exhibit any decrease in viability over the 43 day period. There was a slight increase in cell numbers, from $5 \times 10^{6}$ to $2 \times 10^{7} \mathrm{CFU} / \mathrm{ml}$ within the first 10 days, presumably due to the metabolism of stored material. The cell numbers then remained stable, dropping only to $1.2 \times 10^{7} \mathrm{CFU} / \mathrm{ml}$.

It is interesting to note that, when grown in $\mathrm{LB}$, the viable cell count decreases to approximately the same as that in water. This drop in culturability, of $1-2 \operatorname{logs}$, is similar to that observed in E. coli grown in LB (Siegele et al., 1993) and in Oenococcus oeni grown in modified FT 80 medium, containing meat extract, yeast extract, salts and carbohydrates (Cavin et al., 1989; Zapparoli, 2004). Similarly, Enterococcus faecalis was found to survive multiple-nutrient starvation in water for up to 80 days (Hartke et al., 1998). Mycobacterium tuberculosis and Mycobacterium kansasii were shown to survive for up to 2 years in rich nutrient broth or in distilled water (Nyka, 1974). Survival in water was also observed in E. coli for up to 20 days (Ozkanca \& Flint, 1997), although only when incubated at $4-25^{\circ} \mathrm{C}$. At $30^{\circ} \mathrm{C}$, the culture did not yield colonies on agar after 7 days incubation in water. 


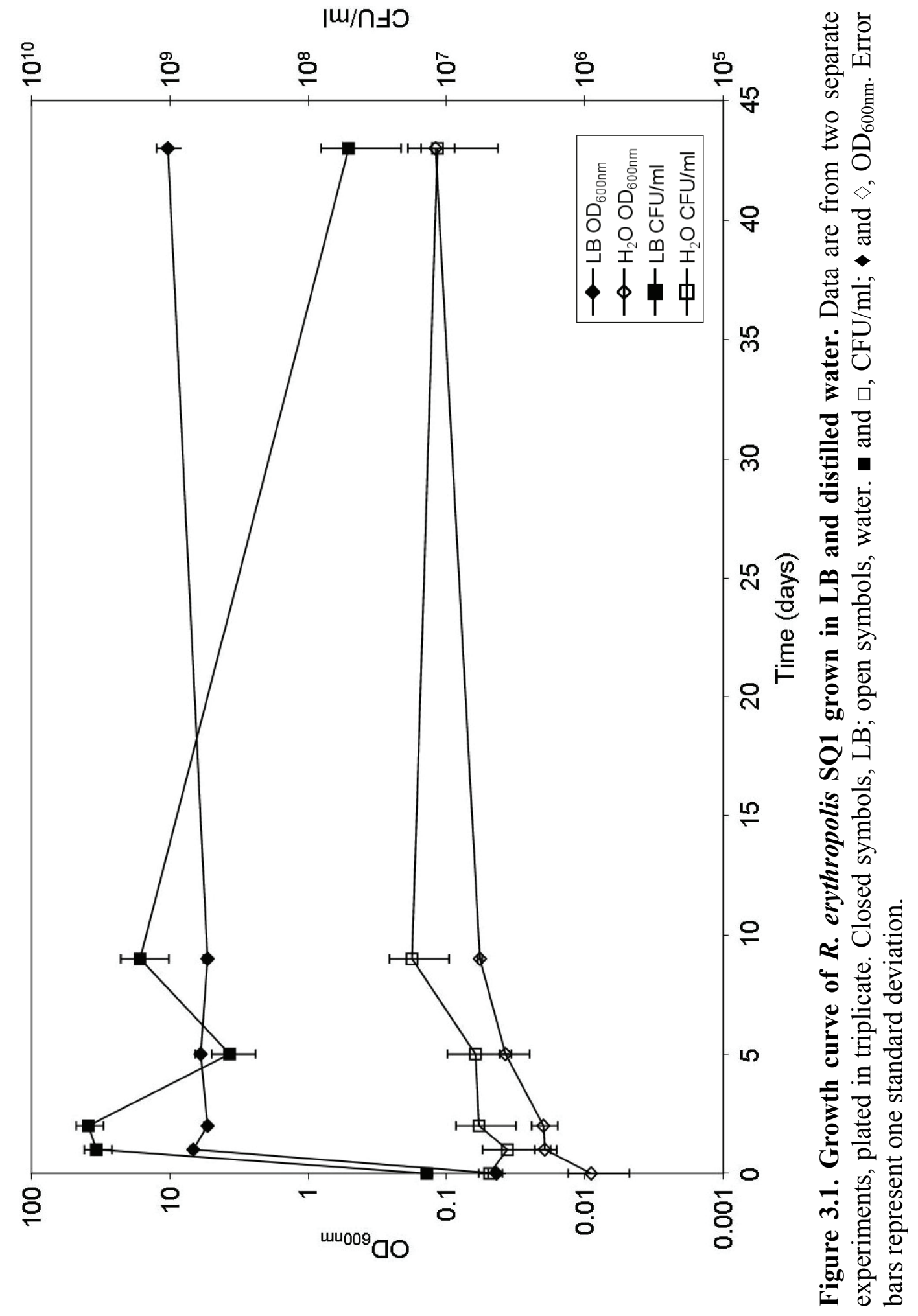




\subsubsection{Response of $R$. erythropolis $S Q 1$ to glucose limitation}

The response of $R$. erythropolis SQ1 to glucose starvation was initially characterised using the experimental medium CDM IA with two different glucose concentrations $1 \%$ and $0.1 \% \mathrm{w} / \mathrm{v}$. Exponential-phase cultures (corresponding to a $24 \mathrm{hr}$ culture) were used to inoculate flasks containing $100 \mathrm{ml}$ of media to a starting $\mathrm{OD}_{600 \mathrm{~nm}}$ of 0.01 . Flasks were then incubated at $27^{\circ} \mathrm{C}$, at $200 \mathrm{rpm}$. Samples were taken at regular intervals over a period of 43 days. For each sample, viable plate counts (CFU/ml) and optical density $\left(\mathrm{OD}_{600 \mathrm{~nm}}\right)$ were determined (Figure 3.2). It should be noted that the flasks were transferred to a static incubator after entry into stationary phase as determined from OD measurements, as described previously in starvation experiments for Vibrio and $S$. aureus (Nyström et al., 1990; Watson et al., 1998b). This corresponds to 7 and 12 days for the $1 \%$ and $0.1 \%$ glucose media, respectively.

The culture grown in CDM IA $0.1 \%$ glucose clearly achieves much lower maximum $\mathrm{OD}_{600 \mathrm{~nm}}(2$ vs 6.6$)$ and viable plate counts $\left(1.3 \times 10^{9}\right.$ vs $\left.6.1 \times 10^{9} \mathrm{CFU} / \mathrm{ml}\right)$ than that grown in $1 \%$ glucose. This confirms that glucose is the limiting component in the medium. It is worth noting that maximum cell numbers were attained after 7 and 14 days of incubation in CDM IA $1 \%$ and $0.1 \%$ glucose, respectively. The calculated growth rate for the culture grown in $1 \%$ glucose was indeed nearly double that of the culture grown in $0.1 \%$ glucose (i.e. $0.17 \mathrm{hr}^{-1}$ vs $0.094 \mathrm{hr}^{-1}$; Figure 3.3). 


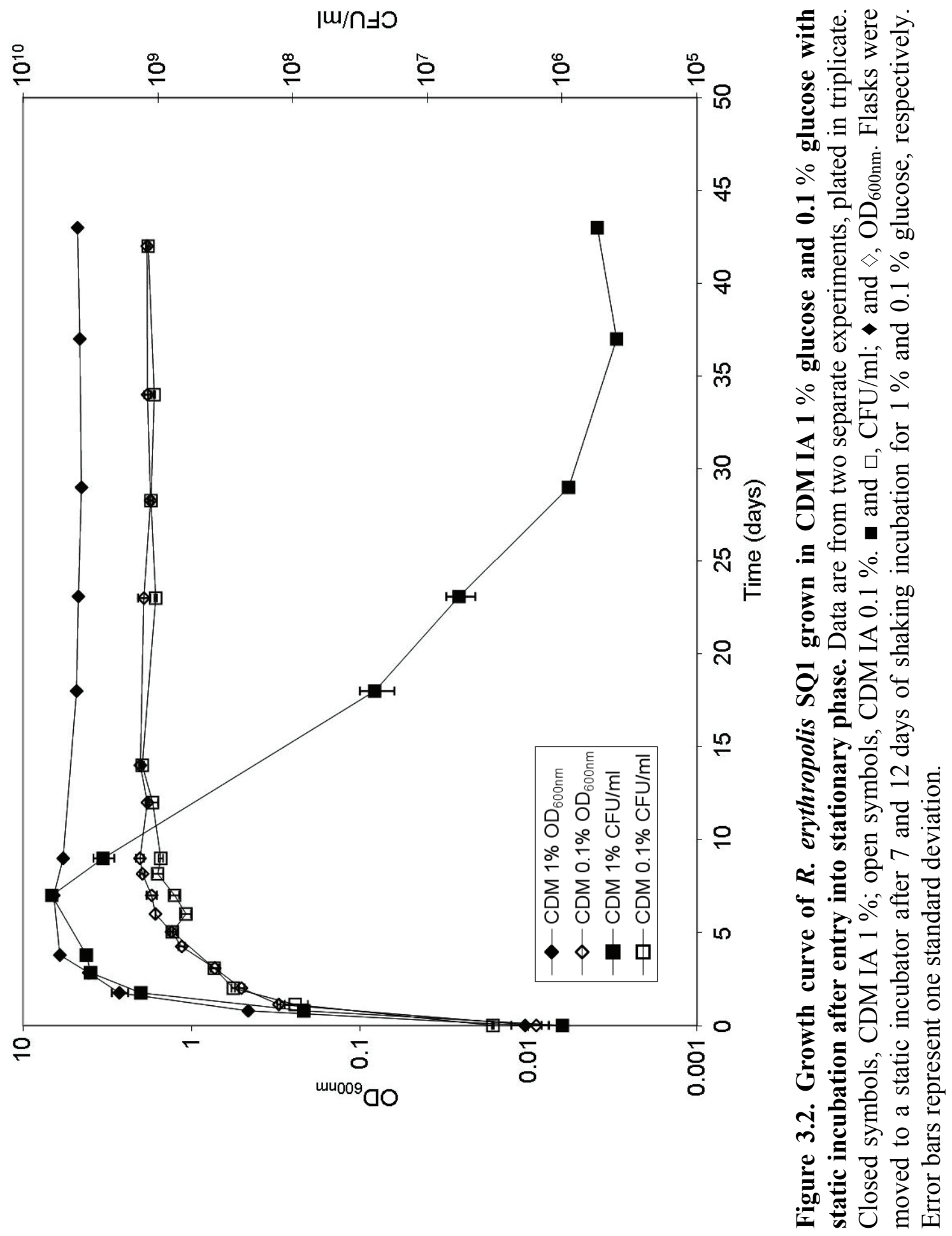




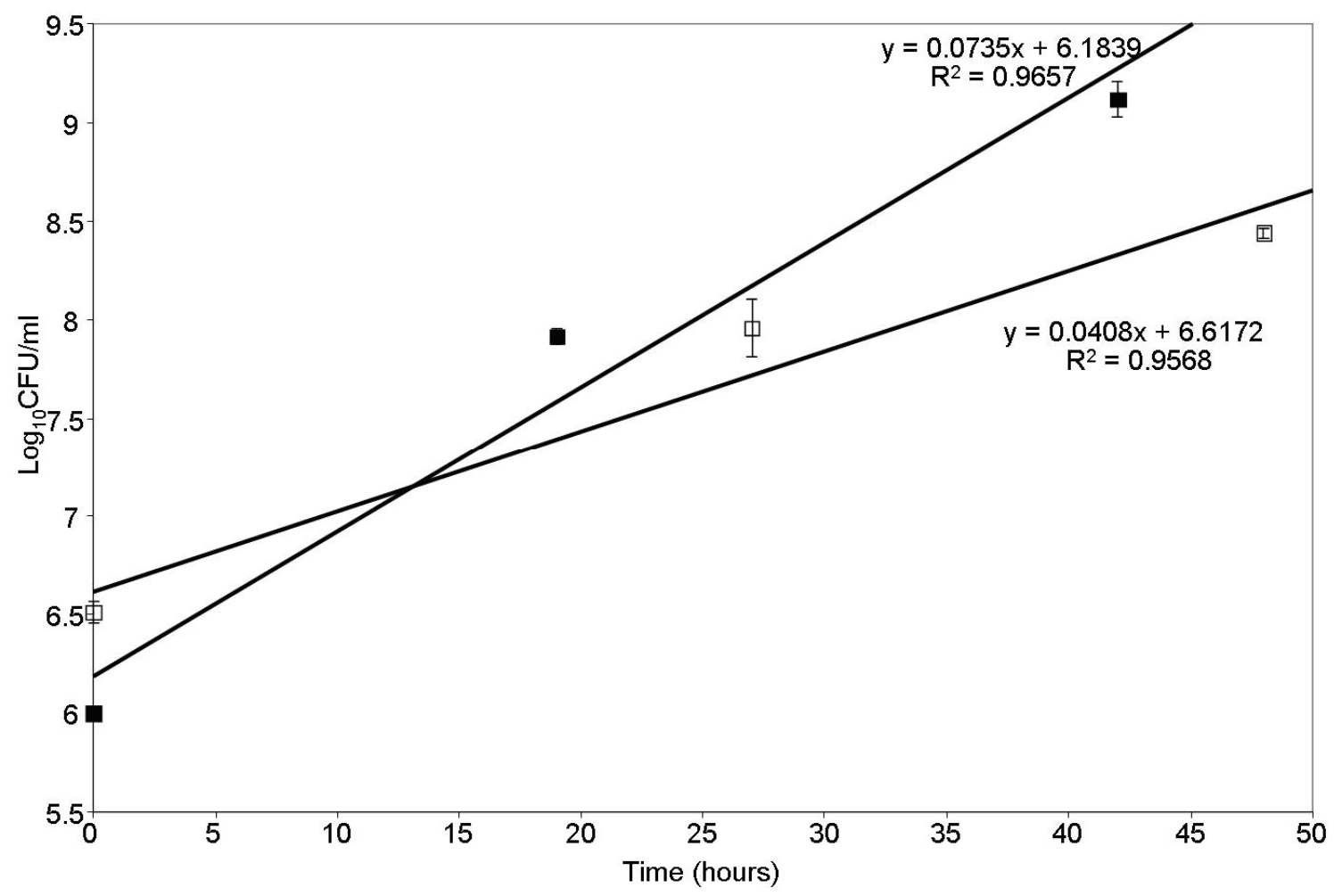

Figure 3.3. Comparison of the growth rates of $R$. erythropolis SQ1 grown in CDM IA $1 \%$ (घ) and $0.1 \%$ glucose (). Data plotted were obtained from two experiments, plated in triplicate. Error bars represent one standard deviation. 
Differences in culture behaviour are quite striking after the logarithmic phase of growth - the culture grown in glucose-limited medium reaches a plateau, whereas the culture grown in non-limiting medium ( $1 \%$ glucose) exhibits a rapid reduction in culturability, as demonstrated by the viable plate counts (Figure 3.2). There is a steady decline in the culturability of the culture, from a maximum of $6.1 \times 10^{9} \mathrm{CFU} / \mathrm{ml}$ to less than $10^{6} \mathrm{CFU} / \mathrm{ml}$.

As mentioned in Section 3.1.1. (Design of a chemically defined medium suitable for studying the effect of carbon starvation on Rhodococcus), the buffering capacity was a concern with CDM IA. To determine whether the drop in viability observed in Figure 3.2 was due to phosphate starvation and/or variation in $\mathrm{pH}$, this experiment was repeated using the improved phosphate-buffered medium CDM IIA with glucose concentrations of $1 \%$ and $0.1 \% \mathrm{w} / \mathrm{v}$. The experiment was conducted as described above with flasks being transferred to static incubation upon entry into the stationary phase, which in the case of the CDM IIA $1 \%$ and $0.1 \%$ glucose corresponded to 10 and 9 days, respectively. There was no significant difference in the behaviour of R. erythropolis SQ1 when grown in CDM IA or CDM IIA (Figure 3.4). 


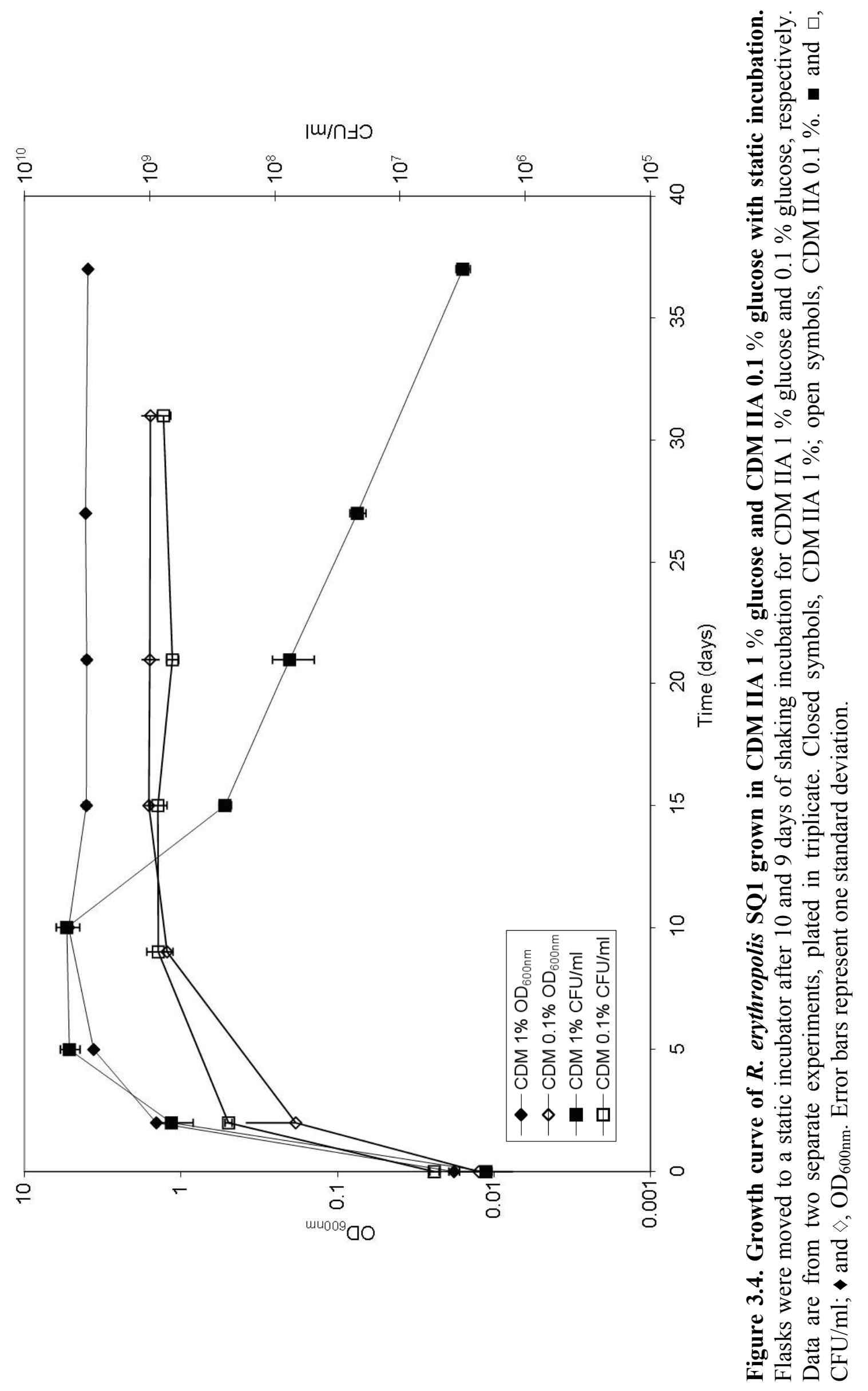


It is worth noting that the drop in culturability observed in Figures 3.2 and 3.4 is coincident with the transfer of the culture from shaking to static incubation. Static incubation was the standard method used in the study of starvation survival of Vibrio and S. aureus (Nyström et al., 1990; Watson et al., 1998b), but interestingly placing flasks of growing cultures in a stationary incubator is the method used to produce dormant Mycobacterium tuberculosis (Wayne \& Hayes, 1996). In order to determine whether transfer to static incubation impacted on the culturability of $R$. erythropolis SQ1, an experiment was performed in which the growth curves of cultures subjected to continuous agitation were compared with cultures transferred to static incubation following 24 hours (mid-exponential) of shaking incubation (Figure 3.5).

Interestingly, when $R$. erythropolis SQ1 was transferred to static incubation after 24 hours of growth, no dramatic drop in CFU/ml was observed (Figure 3.5a). There is a decrease in viable counts from $1.8 \times 10^{9}$ to $1 \times 10^{8} \mathrm{CFU} / \mathrm{ml}$ in CDM IIA $1 \%$ glucose, but that figure then remained constant throughout the remainder of the experiment. The growth curves also showed that $R$. erythropolis SQ1 could survive long-term starvation under aerated conditions (Figure 3.5b). Although there is an initial drop in culturability when $R$. erythropolis SQ1 is grown in $1 \%$ glucose, it occurs comparatively late (at 20 days of incubation) and is less pronounced, i.e. less than 1-log reduction in the viable count, compared to a 4-log reduction when agitation is stopped at early stationary phase (Figure 3.2, Figure 3.5b). Furthermore, the viable count remained constant during subsequent incubation.

Since there was a possibility that the drop in culturability associated with transfer to static incubation was due to depletion of oxygen in the media rather than glucose 
exhaustion, it was decided that all subsequent growth experiments would be performed under agitation. 

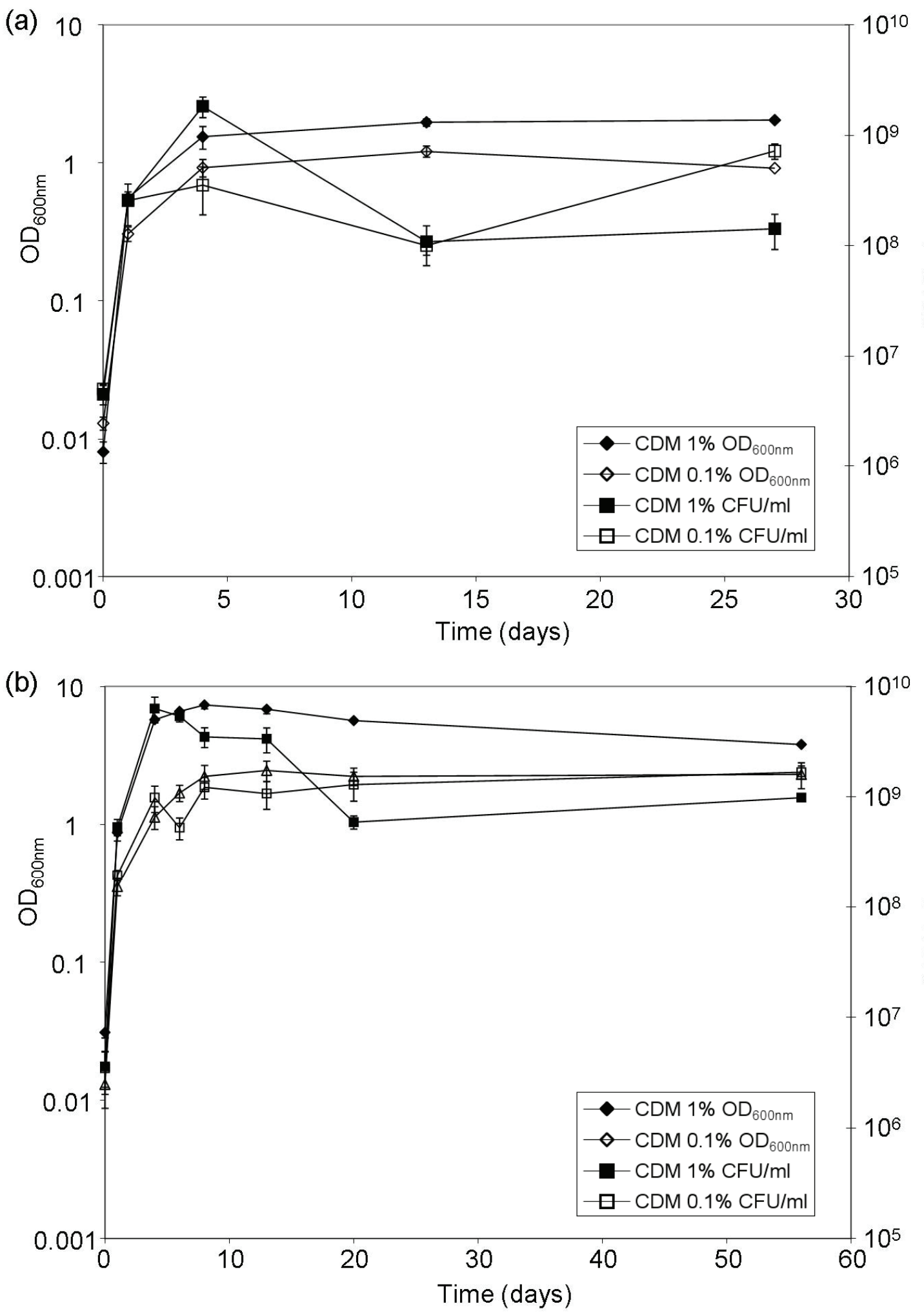

Figure 3.5. Growth curve of $R$. erythropolis SQ1 grown in CDM II $1 \%$ glucose and CDM II $0.1 \%$ glucose with or without static incubation. (a) Flasks were moved to a static incubator after 24 hours of shaking incubation. (b) Flasks were kept in a shaking incubator for the duration of the experiment. Data are from two separate experiments, plated in triplicate. Closed symbols, CDM IIA $1 \%$; open symbols, CDM IIA $0.1 \%$. and $\square, \mathrm{CFU} / \mathrm{ml}$; $\diamond$ and $\diamond, \mathrm{OD}_{600 \mathrm{~nm}}$. Error bars represent one standard deviation. 


\subsubsection{Consumption of glucose in CDM}

The glucose concentration in the growth medium was monitored by a colorimetric method in parallel with optical density and viable plate count measurements. Standard curves were performed to allow for accurate measurement of the glucose concentration (Figure 3.6).

Figure 3.7 demonstrates the utilisation of glucose by $R$. erythropolis SQ1 growing in CDM IIA $1 \%$ glucose (Figure $3.7 \mathrm{a}$ ) and $0.1 \%$ glucose (Figure $3.7 \mathrm{~b}$ ). Note that at 7 days, the glucose is completely exhausted in CDM IIA $0.1 \%$, whereas there is still in excess of $15 \mathrm{mM}$ remaining in $1 \%$ glucose medium. In both cases the glucose concentration drops sharply, by $50 \%$, in just 24 hours. It should also be noted that in both the $1 \%$ and $0.1 \%$ glucose media, the glucose concentration eventually drops to levels that are not detectable by the colorimetric method used $(<0.1 \mathrm{mM})$. 
(a)

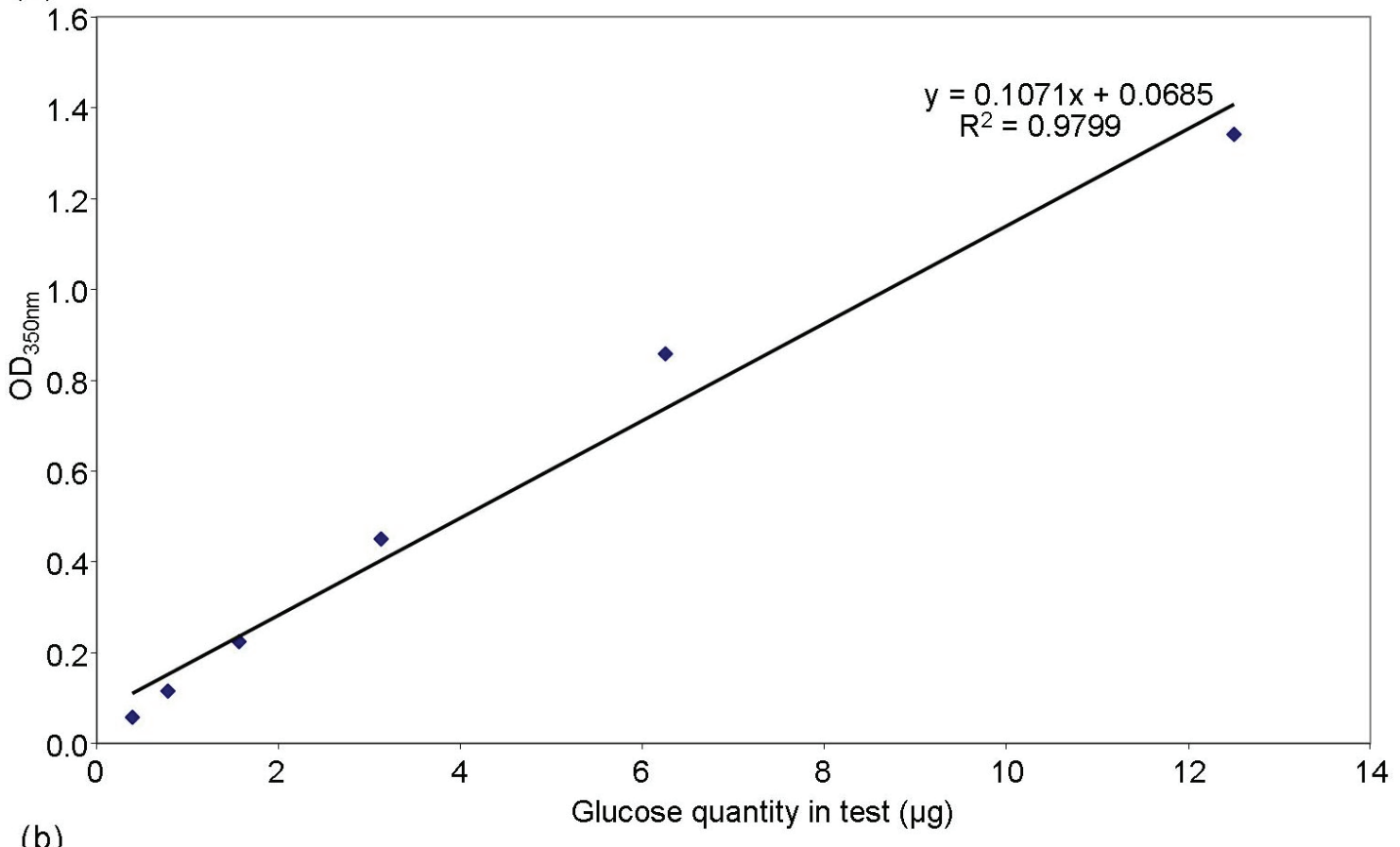

(b)

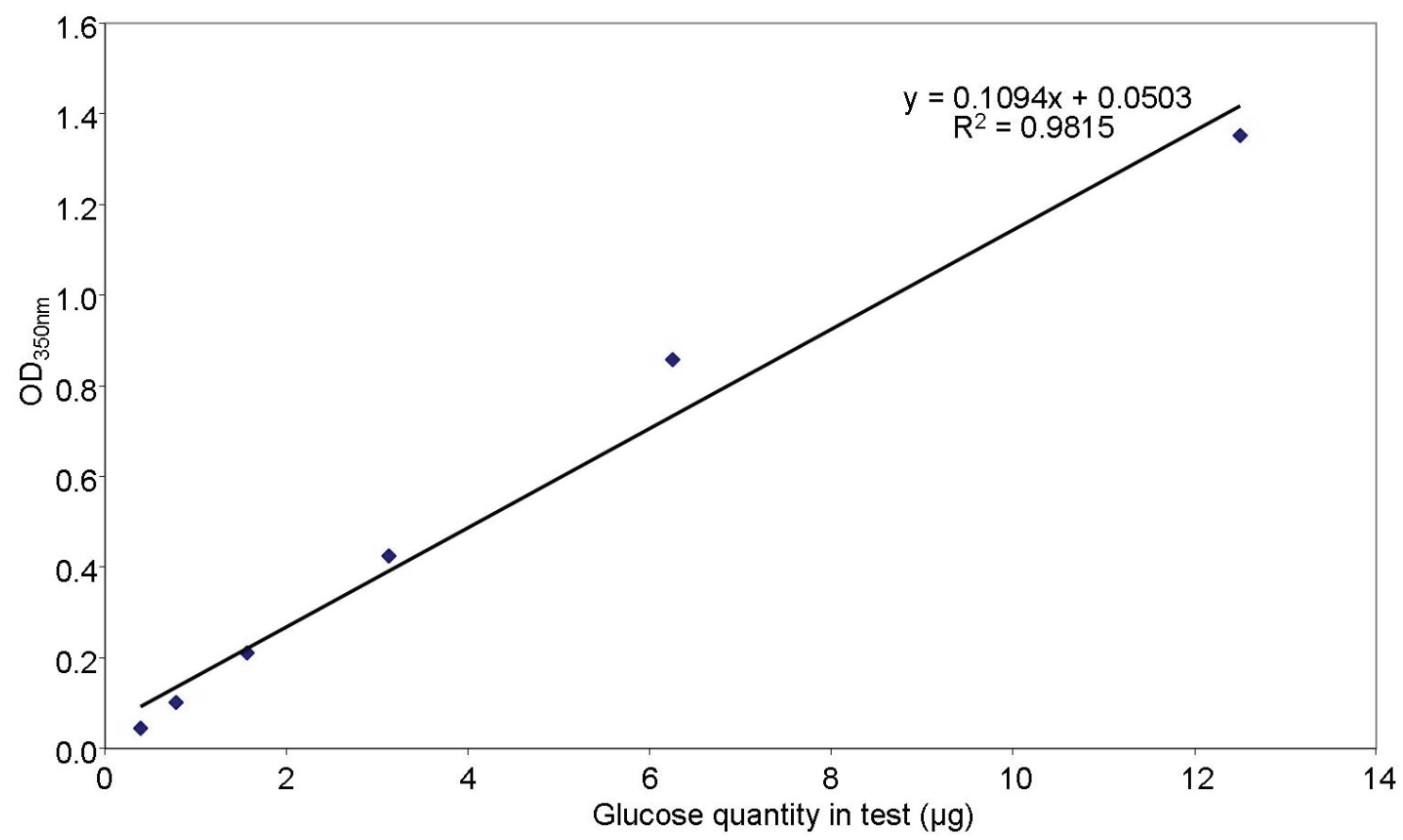

Figure 3.6. Representative standard curves for measurement of glucose in CDM IIA. (a) Standard curve performed with CDM IIA-0.1\% glucose, (b) standard curve performed with CDM IIA-1 \% glucose. 

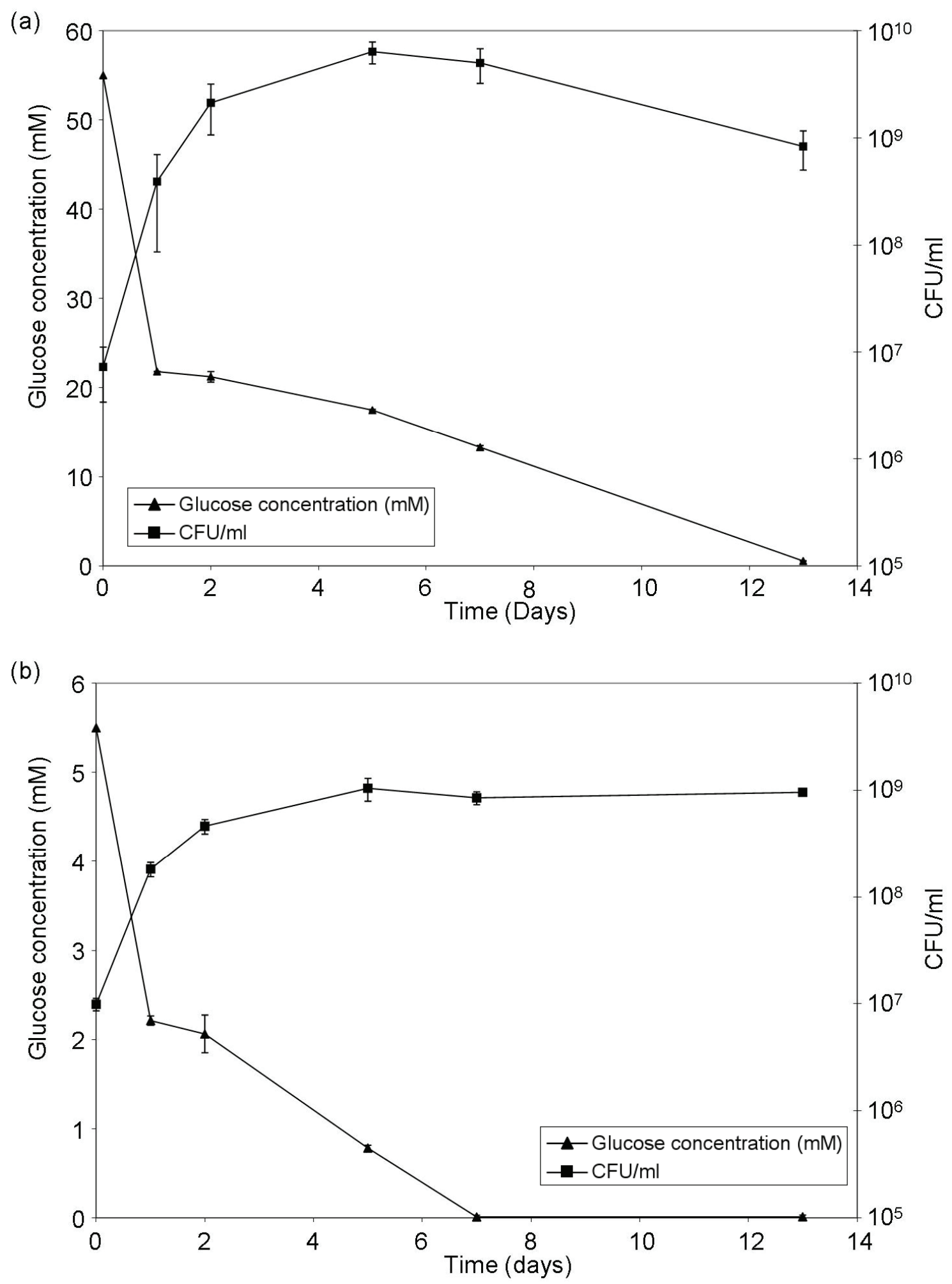

Figure 3.7. Monitoring of glucose concentration during growth of $R$. erythropolis SQ1 in (a) CDM IIA 1\% glucose and (b) CDM IIA 0.1\% glucose.

Data are from two separate experiments, plated in triplicate. $\mathbf{\square}, \mathrm{CFU} / \mathrm{ml} ; \boldsymbol{\Delta}$, glucose concentration in $\mathrm{mM}$. Error bars represent one standard deviation. Note that the glucose concentration ranges up to $55 \mathrm{mM}$ in CDM IIA $1 \%$ glucose, but only $5.5 \mathrm{mM}$ in $0.1 \%$ glucose. 


\subsection{Starvation of R. erythropolis SQ1: Cross protection induced by carbon starvation}

Bacteria in the environment are subjected to permanently changing conditions, especially in terms of nutrient availability and both physical and chemical challenges, including temperature fluctuations and oxidative stress. There have been a number of reports demonstrating a correlation between nutrient starvation and the ability of a bacterium to survive changing environmental conditions. Studies examining the effects of carbon starvation on survival and cross-protection have been performed in vitro on E. coli (Jenkins et al., 1988). Similar observations have been made in a limited number of bacterial genera including Vibrio (Nyström et al., 1992), Pseudomonas putida (Givskov et al., 1994), Salmonella typhimurium (Seymour et al., 1996), Enterococcus faecalis (Giard et al., 1997), Mycobacterium smegmatis (Smeulders et al., 1999) and Listeria monocytogenes (Ferreira et al., 2001). These studies have shown that bacteria starved for carbon developed cross-protection against a range of stress factors. Such observations may be of particular importance to the survival of non-sporulating bacteria. In this study, the effect of glucose starvation on the ability of R. erythropolis SQ1 to survive heat shock and oxidative stress was examined. 


\subsubsection{The effect of carbon-starvation on the ability of $R$. erythropolis SQ1 to survive heat-shock}

CDM IIA $0.1 \%$ glucose was chosen as the growth medium for this experiment in order to ensure that the growth limiting factor was the carbon and energy source. Flasks containing $100 \mathrm{ml}$ of medium were inoculated at a cell density of $0.01 \mathrm{OD}_{600 \mathrm{~nm}}$ with exponential phase cultures (24 hours growth) and incubated at $27^{\circ} \mathrm{C}$ with $200 \mathrm{rpm}$ agitation. Samples were taken at 48 and 144 hrs (2 and 6 days), corresponding to an exponentially growing and an early stationary phase culture, respectively. In order to investigate the protection afforded by carbon limitation to heat shock, the time points for the shock were chosen according to the glucose concentration of the medium, as illustrated previously in Figure 3.7 as days 2 and 6 - at day 2 when the culture is in exponential phase glucose is still plentiful, while day 6 corresponds to 24 hours before exhaustion of glucose from the medium.

In a preliminary experiment, the exponential and early stationary phase samples taken as described above were aliquoted and incubated in a $57^{\circ} \mathrm{C}$ water bath for up to 60 minutes. Aliquots were removed at 5, 10, 15, 20, 30 and 60 minutes and viable plate counts were performed. Percentage survival was determined for each time point as a percentage of the viable count at time of sampling (i.e. a sample not subjected to heat treatment) (Figure 3.8). The early stationary phase culture demonstrated an increased resistance to heat shock in comparison to the exponential phase culture. Since little growth could be observed after 15 minutes heat shock it was decided that, in subsequent experiments, experiments would be performed over 15 minutes with sampling at 5 minute intervals. 


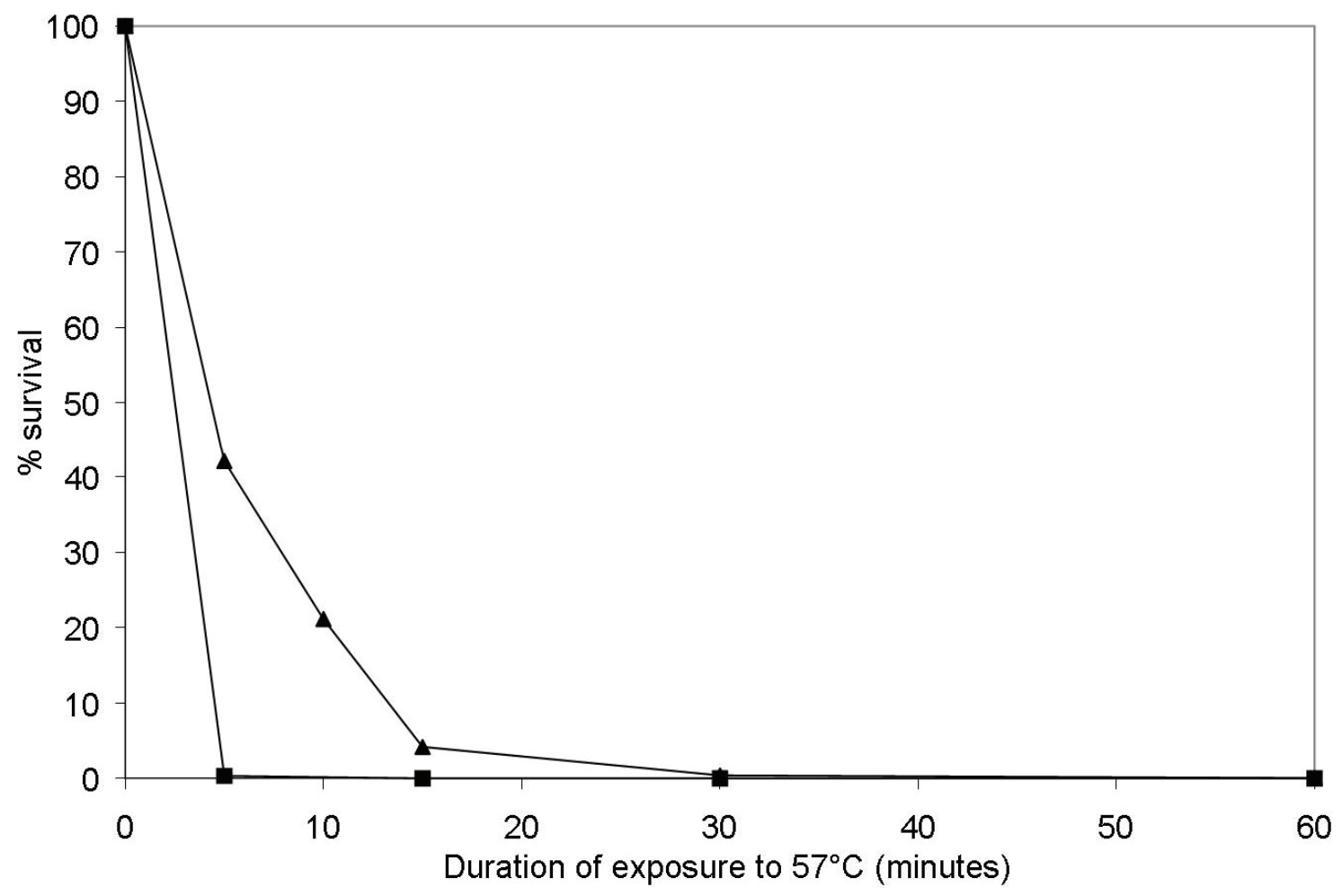

Figure 3.8. Preliminary heat shock experiment in which $R$. erythropolis SQ1 cultures were exposed to heat treatment at $57^{\circ} \mathrm{C}$.

$\mathbf{\square}$, exponential phase culture; $\boldsymbol{\Lambda}$, early stationary phase culture. Results shown represent one experiment. 
In the experiment described above, the cell density of the two cultures being compared (exponential and early stationary phase) obviously differ. Concerns were raised over the effect of cell density on the survival of $R$. erythropolis SQ1 subjected to heat shock. In order to address this issue, a late exponential phase culture was diluted in CDM IIA lacking glucose to approximately $0.5 \mathrm{OD}_{600 \mathrm{~nm}}$ and submitted to heat shock as described above. The same culture was also submitted to the standard protocol described earlier to provide a control. No significant difference could be observed in the ability to survive heat shock (Figure 3.9), showing that cell density is not a significant factor in the resistance of $R$. erythropolis SQ1 to heat shock. Further experiments were therefore performed without diluting the cells prior to heat shock. 


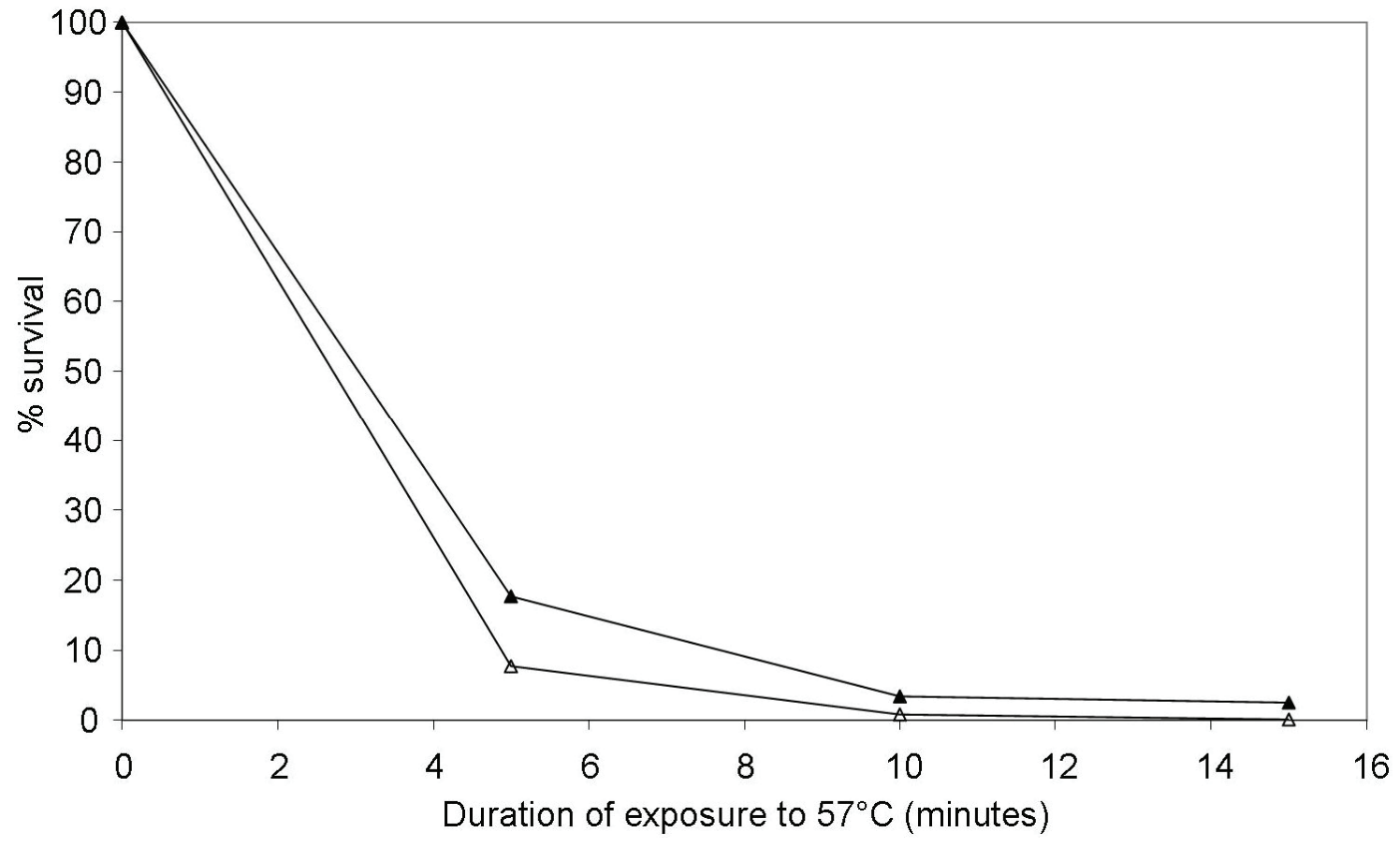

Figure 3.9. Effect of heat shock on diluted and undiluted late exponential phase cultures of $\boldsymbol{R}$. erythropolis SQ1. $\triangle$, late exponential phase culture (approx. $\left.2.0 \mathrm{OD}_{600 \mathrm{~nm}}\right) ; \boldsymbol{\Delta}$, late exponential phase diluted to approximately $0.5 \mathrm{OD}_{600 \mathrm{~nm}}$. 
Chloramphenicol is a bacteriostatic antibiotic that inhibits protein synthesis by blocking the peptidyl transferase activity of the ribosome subunit 50S (Neu \& Gootz, 1996). To investigate whether the observed cross-protection induced by carbon starvation could be reduced by inhibiting protein synthesis, the challenged cultures were incubated for $2 \mathrm{~h}$ with $40 \mu \mathrm{g} \mathrm{ml}^{-1}$ chloramphenicol prior to heat shock treatment. This chloramphenicol concentration was selected as it is the one at which $R$. erythropolis SQ1 growth is inhibited, as illustrated in Table 3.2, thus ensuring that protein synthesis is inhibited.

In order to investigate the effect of protein synthesis inhibition on heat shock survival, samples were taken of exponential and early stationary phase cultures as described above, aliquoted and either treated with chloramphenicol or kept at $27^{\circ} \mathrm{C}$ for 2 hours. All samples were then incubated in a $57^{\circ} \mathrm{C}$ water bath for up to 15 minutes. At 5 minute intervals, aliquots were removed and viable plate counts were performed. Percentage survival was determined for each time point as a percentage of the viable count at time of sampling (Figure 3.10).

Figure 3.10 shows very clearly that early stationary phase cells are more resistant to heat shock than exponential phase cells, with $40-50 \%$ cells surviving 5 minutes of shock vs $0 \%$, respectively. Interestingly, treatment of the cultures with $40 \mu \mathrm{g} \mathrm{ml}^{-1}$ chloramphenicol prior to heat shock treatment did not have any substantial effect on the resistance of $R$. erythropolis SQ1 to heat shock, in that their resistance to heat shock was not diminished. It has previously been shown for $E$. coli that when cultures were treated with $100 \mu \mathrm{g} \mathrm{ml}^{-1}$ chloramphenicol for approximately 4 hours after depletion of glucose from the growth medium, stationary phase cells were as sensitive to heat shock as exponentially growing cells (Jenkins et al., 1988). Inhibition of heat shock resistance by chloramphenicol has also been demonstrated for Enterococcus faecalis (Giard et al., 
1996) and Clostridium perfringens (García et al., 2001), although the heat-shock resistance in Clostridium was not starvation-induced. It was therefore hypothesised that in E. coli and Enterococcus, proteins are synthesised upon entry into stationary phase which confer heat resistance capabilities to the cells and that treatment with chloramphenicol blocks the development of heat resistance by blocking new protein synthesis. 


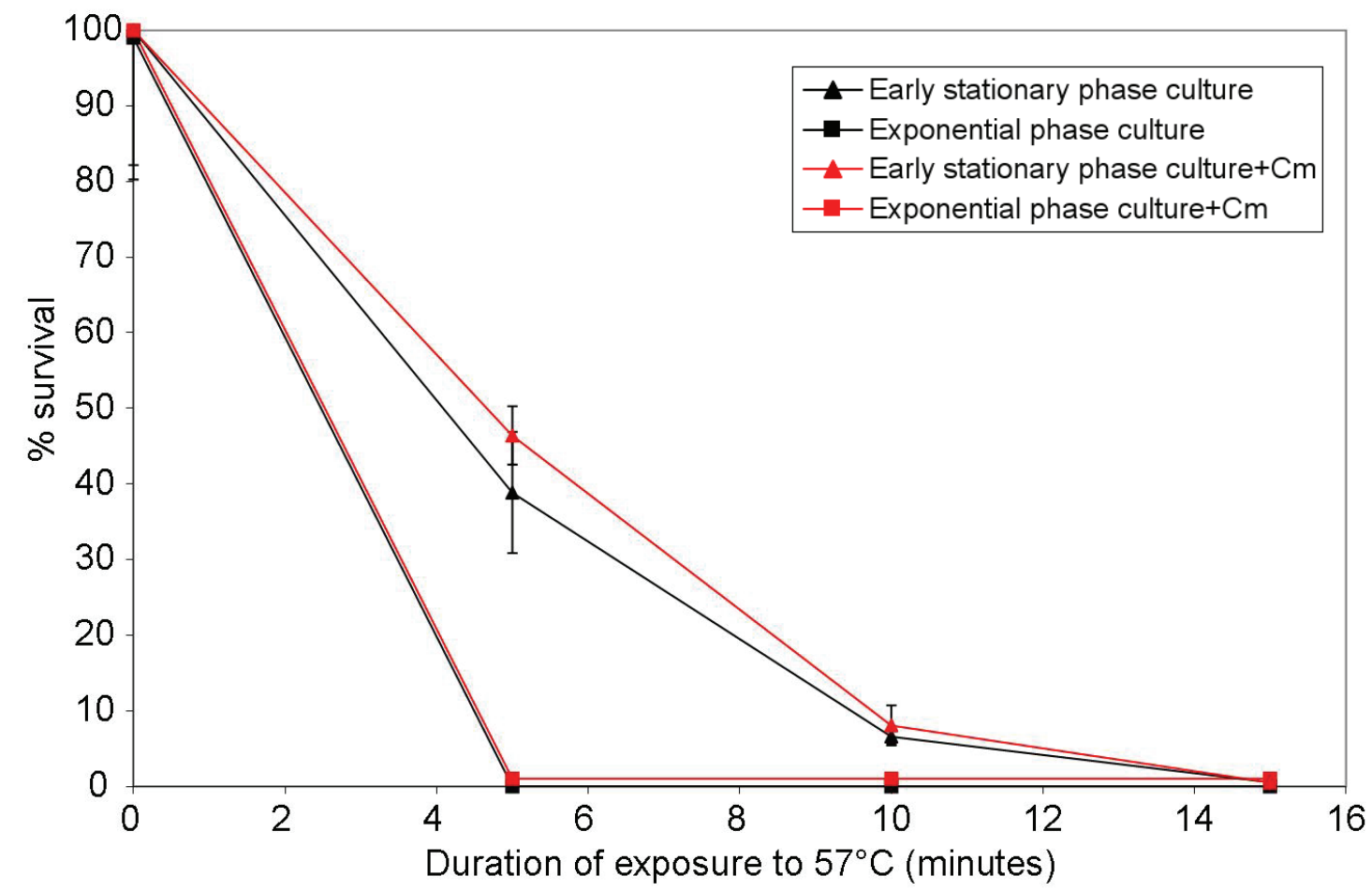

Figure 3.10. Survival of $R$. erythropolis SQ1 subjected to heat shock $\left(57^{\circ} \mathrm{C}\right)$ with and without exposure to $40 \mu \mathrm{g} \mathrm{ml}^{-1}$ chloramphenicol.

-, exponential phase culture; $\square$, exponential phase culture treated with chloramphenicol; $\boldsymbol{\Delta}$, early stationary phase culture; $\boldsymbol{\Delta}$, early stationary phase culture treated with chloramphenicol. Error bars represent one standard deviation. 


\subsubsection{Cross-protection against oxidative stress}

In studies performed to date examining the interaction between nutrient starvation and the development of resistance to oxidative stress, hydrogen peroxide $\left(\mathrm{H}_{2} \mathrm{O}_{2}\right)$ is typically used as the oxidative agent (see for example Giard et al., 1996; Jenkins et al., 1988). $\mathrm{H}_{2} \mathrm{O}_{2}$ was initially used in this study to induce oxidative stress, but the standard deviations obtained were so large that the results were not significant (data not shown). Additionally, it has been reported that $R$. erythropolis is able to use $\mathrm{H}_{2} \mathrm{O}_{2}$ as a sole oxygen source (Tarasov et al., 2004). Other oxidative agents that have been used in oxidative damage studies reported in the literature include paraquat, menadione and tert-butyl hydroperoxide (tBHP). tBHP was used in oxidative damage studies with Pseudomonas aeruginosa (Ochsner et al., 2000) and, in preliminary experiments performed in this study, tBHP was found to give reliable and reproducible results.

A preliminary experiment was performed to estimate the quantity of tBHP necessary to induce measurable cell death. Exponentially growing $R$. erythropolis SQ1 cells were washed in saline and resuspended to approximately $1 \times 10^{9} \mathrm{CFU} / \mathrm{ml}$. They were then exposed to an increasing concentration of tBHP in the 1 to $600 \mathrm{mM}$ range. After 5 minutes, the cells were diluted by serial dilution (hence also diluting tBHP) and plated in triplicate on LB agar in order to obtain viable plate counts (Figure 3.11). $400 \mathrm{mM}$ of tBHP gave a drop in culturability of approximately 3 logs, which was deemed adequate for the purpose of further experiments. 


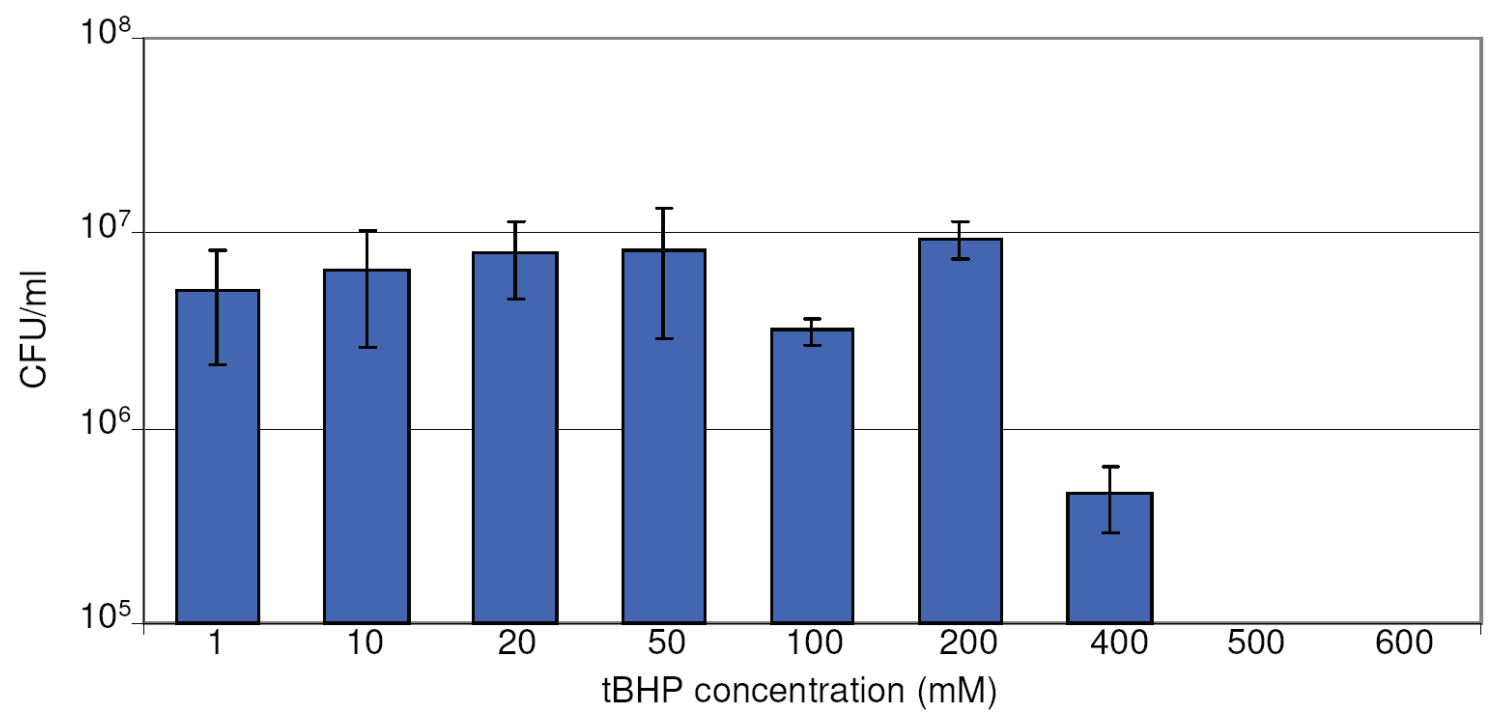

Figure 3.11. Effect of increasing concentrations of tBHP on exponentially growing R. erythropolis SQ1 cells.

At each concentration approximately $1 \times 10^{9} \mathrm{CFU} / \mathrm{ml}$ was treated with tBHP for $5 \mathrm{~min}$ and then plated in triplicate. No growth was observed at 500 and $600 \mathrm{mM}$ tBHP. Results presented are that of one experiment. 
Having established the concentration of tBHP required to induce oxidative stress, the effect of carbon starvation on oxidative stress resistance was investigated. CDM IIA $0.1 \%$ glucose was chosen as the growth medium for this experiment in order to ensure that the growth limiting factor was the carbon and energy source. Flasks containing $100 \mathrm{ml}$ of medium were inoculated at a cell density of $0.01 \mathrm{OD}_{600 \mathrm{~nm}}$ with exponential phase $(24 \mathrm{~h})$ cultures and incubated at $27^{\circ} \mathrm{C}$ with $200 \mathrm{rpm}$ agitation. Samples were taken at 48 and 144 hours (2 and 6 days), corresponding to exponentially growing and early stationary phase cultures, respectively. In order to investigate the protection afforded by carbon limitation to oxidative stress, the time points for the shock were chosen according to the glucose concentration of the medium, as illustrated previously in Figure 3.7 as days 2 and $6-$ at day 2 when the culture is in exponential phase and glucose is still plentiful, while day 6 corresponds to 24 hours before exhaustion of glucose from the medium.

The exponential and early stationary phase samples taken above were aliquoted, washed and adjusted to $1 \times 10^{6} \mathrm{CFU} / \mathrm{ml}$ in saline, as described in Chapter II. Aliquots were then subjected to oxidative shock by the addition of tBHP at a final concentration of $400 \mathrm{mM}$ and samples were incubated at $27^{\circ} \mathrm{C}$ and under agitation. The percentage survival of the cells was then determined from viable plate counts in which samples were diluted in saline and plated in triplicate on LB agar. Percentage survival was calculated as percentage of the viable count of cells that had been removed from the reaction vessel prior to addition of tBHP.

To investigate whether the extent of cross-protection induced could be reduced by inhibiting protein synthesis, the cultures challenged were also incubated for 2 hours with $40 \mu \mathrm{g} \mathrm{ml}^{-1}$ chloramphenicol. Immediately after sampling and dilution, two 
aliquots for each time point were incubated at $27^{\circ} \mathrm{C}$ - one aliquot was treated with chloramphenicol, whereas the other was not. This concentration was selected as it is the one at which $R$. erythropolis SQ1 growth is inhibited, thus ensuring that protein synthesis is inhibited. Oxidative stress was then performed as described above.

At all time points early stationary phase (6 days) $R$. erythropolis SQ1 cultures were found to be more resistant to oxidative stress than exponential phase ( 2 days) cultures, indicating that carbon starvation induced oxidative stress resistance in $R$. erythropolis SQ1 (Figure 3.12). At 10 minutes of exposure, early stationary phase cultures demonstrated $40 \%$ survival in comparison with only $7 \%$ survival for exponential phase cultures. After 15 minutes of exposure to oxidative stress, no exponential phase cells survived, in contrast to $20 \%$ survival for the early stationary phase culture.

In contrast to the results obtained for the heat shock experiment, resistance to oxidative stress was transitorily decreased by treatment with chloramphenicol (Figure 3.12). This effect could be seen only after 5 minutes of oxidative stress; there was no significant difference in percentage survival between chloramphenicol-treated and untreated cells at 10 and 15 minutes. At 5 minutes, the percentage survival was reduced from 48 (no chloramphenicol treatment) to $26 \%$ (chloramphenicol-treated) for exponential phase cultures, and from 63 (no chloramphenicol treatment) to $45 \%$ chloramphenicol-treated) for stationary phase cultures. The same observation was repeatedly made in four independent experiments. 


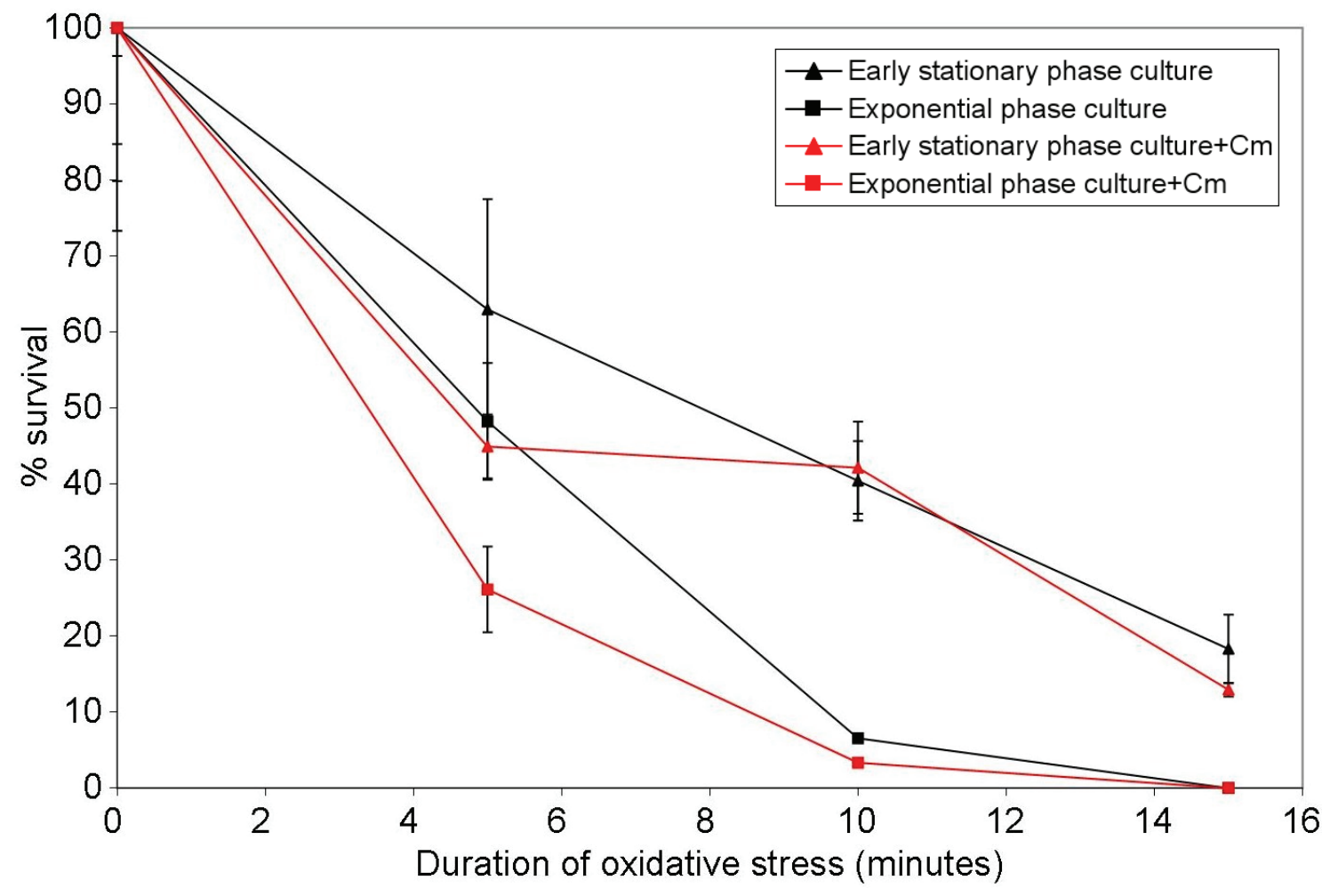

Figure 3.12. Survival of $R$. erythropolis SQ1 subjected to oxidative stress (400 $\mathrm{mM}$ tBHP) with and without exposure to $40 \mu \mathrm{g} \mathrm{ml}^{-1}$ chloramphenicol.

$\mathbf{\square}$, ; $\mathbf{m}$, exponential phase $R$. erythropolis SQ1 culture treated with chloramphenicol; $\boldsymbol{\Delta}$, early stationary phase $R$. erythropolis SQ1 culture; $\boldsymbol{\Delta}$, early stationary phase $R$. erythropolis SQ1 culture treated with chloramphenicol. 


\section{Chapter IV}

\section{Random Transposon Mutagenesis of $R$. erythropolis SQ1 and Genomic Analysis of the Mutants}


Mutagenesis, both random and site-directed, is an essential tool in the functional analysis of microbial genomes. Many biochemical pathways and mechanisms have been elucidated by studying mutants. An example is the histidine synthesis pathway of E. coli (Haas et al., 1952). In the context of understanding starvation survival, mutagenesis strategies have been used in Listeria monocytogenes (Herbert \& Foster, 2001), Staphylococcus aureus (Watson et al., 1998a) and Mycobacterium smegmatis (Keer et al., 2000), amongst others. These studies used random mutagenesis based on transposons. Transposon mutagenesis provides many advantages over other mutagenesis methods. Most transposon systems will allow only one insertion to take place at a time, therefore disabling a single gene or a limited area of the genome (Berg et al., 1983). This is in contrast to physical (UV) or chemical methods (e.g. with methoxylamine or $N^{\prime}$-methyl- $N^{\prime}$-nitro- $N$-nitrosoguanidine) that affect the entirety of the genome. The transposon used to disrupt the target DNA also usually contains an antibiotic resistance gene, so that mutants are easily selected. Since the sequence of the transposon is known, it also allows for recovery of the flanking sequences by various PCR methods. One drawback is that the insertion of the transposon is influenced by the target sequence, e.g. in vitro the transposon $\mathrm{Tn} 5$ inserts preferentially in $\mathrm{G}+\mathrm{C}$-rich areas of the genome (Herron et al., 2004).

Transposon mutagenesis has been reported for a number of Rhodococcus species. Mutagenesis using a modified Himarl transposon has been reported for R. equi (Ashour \& Hondalus, 2003). pTNR, based on IS1415, was used to mutagenise R. erythropolis, $R$. fascians, $R$. rhodochrous and $R$. ruber (Sallam et al., 2006). Transposon mutagenesis based on the use of the Ez:Tn $<\mathrm{KAN}-2>\mathrm{Tnp}$ transposome from Epicentre has been reported for a number of Rhodococcus species. In this commercial system, the transposome consists of the $19 \mathrm{bp}$ inverted repeat sequences of $\operatorname{Tn} 5$ and the $\operatorname{Tn} 903$ 
kanamycin resistance gene as a complex with the $\operatorname{Tn} 5$ transposase. The transposome is ready to be electroporated into the bacterial cell, where the intracellular $\mathrm{Mg}^{2+}$ activates the transposase. The Ez::Tn5 transposon is then inserted randomly into the genome. Due to the fact that the Ez::Tn5 DNA is not on a carrier plasmid, nor is it an intact transposon, introduction of multiple copies in the genome is limited and indeed unlikely (Goryshin \& Reznikoff, 1998). To date, the use of the EZ::Tn5 transposome has been reported for mutagenesis of Rhodococcus rhodochrous CW25, Rhodococcus erythropolis KA2-5-1 and Rhodococcus equi ATCC 33701 (Fernandes et al., 2001; Mangan \& Meijer, 2001; Tanaka et al., 2002). This transposome system was therefore chosen in this study to generate a mutant bank of $R$. erythropolis SQ1 for the purpose of isolating carbon starvation or stationary phase survival mutants. 


\subsection{Generation of a mutant bank of $R$. erythropolis $S Q 1$}

The protocol described by Tanaka et al. (2002) was used for mutagenesis of R. erythropolis SQ1 with EZ::TN $<\mathrm{KAN}-2>\mathrm{Tnp}$ and resulted in a mutant bank of 898 mutants. This protocol was selected over others because growth of $R$. erythropolis SQ1 in LB supplemented with glycine, as performed by Fernandes et al. (2001) and Mangan \& Meijer (2001) was extremely unpredictable and impeded the production of competent cells. Growth could be very quick or plateau around $\mathrm{OD}_{600 \mathrm{~nm}} 0.5$, and it was not possible to predict whether growth would be fast or slow. A transformation efficiency of $4.5 \times 10^{4}$ mutants $\mu^{-1}$ of transposome DNA was obtained, which compares favourably with the efficiencies reported in the literature of $3 \times 10^{4}$ mutants $\mu \mathrm{g}^{-1}$ for

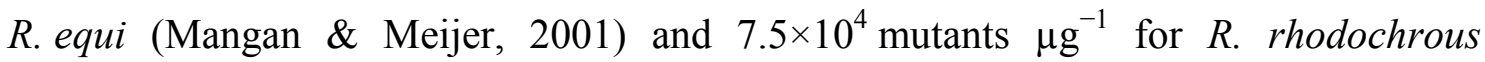
(Fernandes et al., 2001). A higher efficiency of $2.3 \times 10^{6}$ mutants $\mu \mathrm{g}^{-1}$ was reported for R. erythropolis KA2-5-1 (Tanaka et al., 2002), possibly explained by the lower concentration of kanamycin used to select for mutants $\left(100 \mu \mathrm{g} \mathrm{ml}^{-1}\right.$ in contrast to $200 \mu \mathrm{g} \mathrm{ml}^{-1}$ in this study). A higher kanamycin concentration of $200 \mu \mathrm{g} \mathrm{ml}^{-1}$ was needed for $R$. erythropolis SQ1 in order to avoid isolation of spontaneously resistant mutants. The isolation of spontaneous resistant mutants below $400 \mu \mathrm{g} \mathrm{ml}^{-1}$ kanamycin was reported for $R$. rhodochrous by Fernandes et al. (2001).

In order to ensure that resistance to kanamycin was due to insertion of the $\operatorname{Tn} 903$ kanamycin resistance gene in the genome of $R$. erythropolis SQ1, primers KANR-FP and KANR-RP were designed to amplify the kanamycin resistance gene in its entirety. A PCR amplification product of $816 \mathrm{bp}$ would be expected. The plasmid pRE-7 (Zheng et al., 1997), which contains the Tn903 kanamycin resistance gene, was used as a positive control. Agarose gel electrophoresis of the PCR products obtained from 20 randomly selected mutant colonies revealed a band of approximately $800 \mathrm{bp}$ 
(Figure 4.1). This confirms the insertion of the transposome DNA in the Rhodococcus genome. No spontaneously kanamycin-resistant mutants were obtained. In total 898 kanamycin-resistant colonies were obtained. 


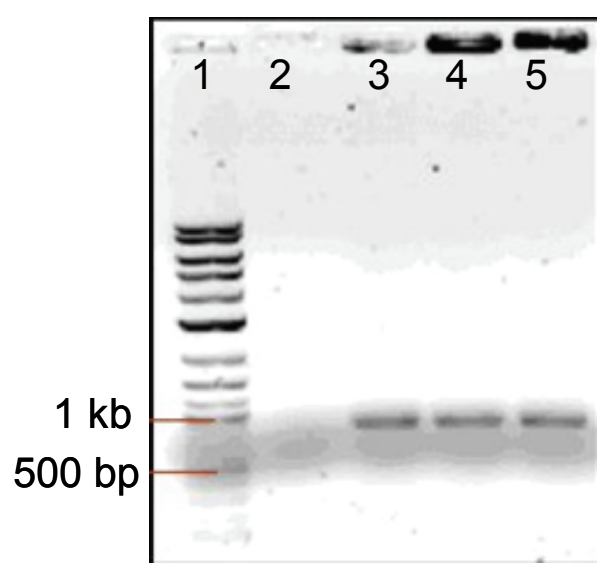

Figure 4.1. Agarose gel (0.8 \%) electrophoresis of the PCR products using KANRFP and KANR-RP, designed to the Tn903 kanamycin resistance gene.

Lane 1, 2-log ladder (New England Biolabs); lane 2, negative control (no DNA); lane 3, positive control (pRE-7); lane 4, mutant 1; lane 5, mutant 2. 


\subsection{Mutant screening strategy}

The mutant bank generated by transposon insertion was screened for mutants affected in stationary phase survival or recovery. This was done following the method detailed by Uhde et al. (1997). Following electroporation, each colony growing on LB agar supplemented with $200 \mu \mathrm{g} \mathrm{ml}^{-1}$ kanamycin was inoculated in an individual well of a microtitre plate containing $200 \mu 1$ kanamycin-supplemented LB broth; 898 mutants were obtained in total. After overnight incubation at $27^{\circ} \mathrm{C}$, the mutants were transferred to a fresh microtitre plate using 96-prong replicators, and the original plate was frozen at $-80{ }^{\circ} \mathrm{C}$ as the master stock. The mutants were again allowed to grow overnight before inoculating microtitre plates containing chemically defined medium CDM IIA 1 and $0.1 \%$ glucose for stationary phase survival and recovery screening. Cultures were never allowed to enter stationary phase except for specific experiments. The mutants, while still in mid-exponential phase, were spotted on LB agar using 96-prong replicators; it was obvious after $48 \mathrm{~h}$ growth that important changes had occurred. The extent of growth on agar varied widely from less than $1 \mathrm{~mm}$ to approximately $10 \mathrm{~mm}$ in diameter. The shape varied slightly, flat growth in particular was observed (Figure 4.2). To facilitate the description and the screening of the mutants, they were classified at this stage in five categories, based on appearance of growth on solid medium (Table 4.1). 


\begin{tabular}{|c|c|}
\hline Group of mutants & Characterisation of growth \\
\hline$\alpha$ & $\begin{array}{l}\text { Identical or similar to wild-type } R \text {. erythropolis SQ1, rough and } \\
\text { matt, slight variation in size and appearance of edge, } \\
\text { approximately } 2 \mathrm{~mm} \text { diameter }\end{array}$ \\
\hline$\beta$ & $\begin{array}{l}\text { Extensive growth, rough and matt, thinning towards edge } \\
\text { (mycelial-like), serrated edges, approximately } 10 \mathrm{~mm} \text { diameter }\end{array}$ \\
\hline$\gamma$ & $\begin{array}{l}\text { Less growth than wild-type, smooth and shiny, very often } \\
\text { irregular shape and well-defined edges, } 1 \text { to } 2 \mathrm{~mm} \text { diameter }\end{array}$ \\
\hline$\delta$ & $\begin{array}{l}\text { Slow growing, appearance often variable, less than } 1 \mathrm{~mm} \\
\text { diameter }\end{array}$ \\
\hline$\varepsilon$ & Increased/decreased pigmentation \\
\hline
\end{tabular}

Table 4.1. Classification of $R$. erythropolis SQ1 mutants on the basis of appearance of growth on agar plates when spotted from microtitre plates. 


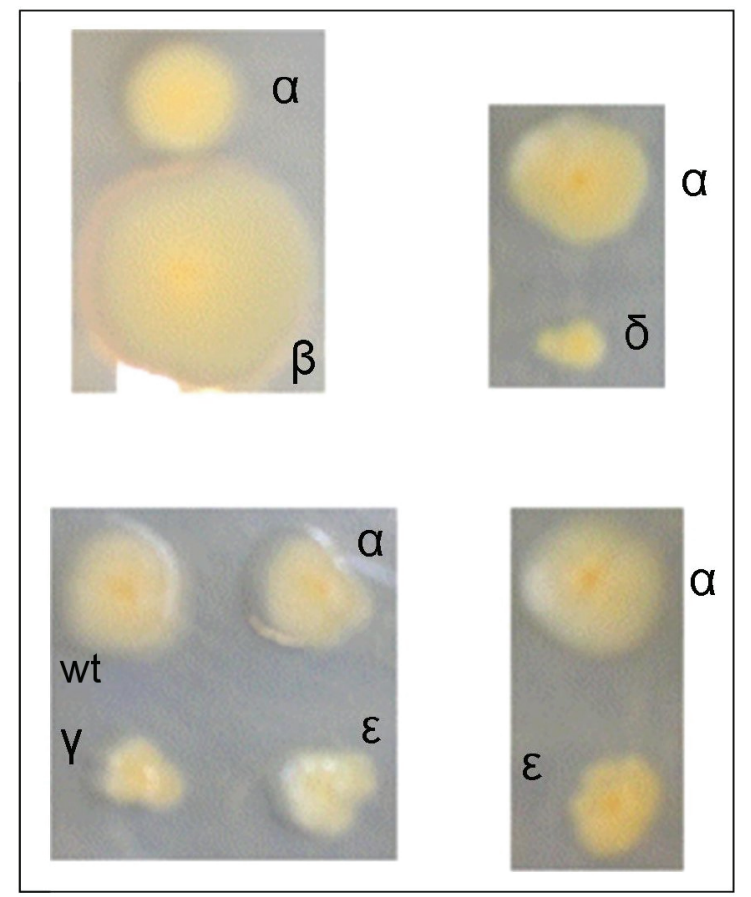

Figure 4.2. Appearance of mutants growing on CDM IIA $1 \%$ glucose agar.

The class of each mutant is indicated on each photo. Growth on LB agar is identical to that on CDM II agar. wt, Wild-type. 
The appearance of $\beta$ mutants on solid medium generally remained constant, in that once the phenotype was observed; it was then reproduced and was not a transitory phase. The growth characteristics observed were also the same whether the mutants were spotted or streaked on agar. The same appearances were observed on CDM II $1 \%$ glucose agar and LB agar. Streaking of mutants on plates also showed that the populations were homogeneous.

To investigate whether the appearance of growth on agar correlated with a change in the appearance of growth in broth, mutants of each category were picked from CDM II $1 \%$ agar plates and inoculated into CDM II $1 \%$ glucose broth. Appearance of growth is noted in Table 4.2. The only obvious differences from the wild-type were observed with mutants of type $\beta$, which aggregated in broth compared to the wild-type $R$. erythropolis SQ1. This may possibly be due to a change in the hydrophobicity of the cell membrane. The same appearance was observed with mutants picked from LB agar plates. 


\begin{tabular}{|c|c|c|c|}
\hline \multirow[t]{2}{*}{ Class of } & \multicolumn{3}{|c|}{$\begin{array}{l}\text { Growth in liquid medium (CDM II } 1 \% \text { glucose broth) when } \\
\text { transferred from CDM II } 1 \% \text { glucose agar }\end{array}$} \\
\hline & $24 \mathrm{~h}$ incubation & $48 \mathrm{~h}$ incubation & $>96 \mathrm{~h}$ incubation \\
\hline$\alpha$ & $\begin{array}{l}\text { Small aggregates (less } \\
\text { than } 0.5 \mathrm{~mm} \text { ) }\end{array}$ & $\begin{array}{l}\text { Predominantly } \\
\text { homogenous growth }\end{array}$ & $\begin{array}{l}\text { Predominantly } \\
\text { homogenous growth }\end{array}$ \\
\hline$\beta$ & $\begin{array}{l}\text { Aggregates (up to } \\
1 \mathrm{~mm} \text { diameter) }\end{array}$ & $\begin{array}{l}\text { Aggregates (up to } \\
1 \mathrm{~mm} \text { diameter) }\end{array}$ & $\begin{array}{l}\text { Aggregates (up to } 1 \mathrm{~mm} \\
\text { diameter) }\end{array}$ \\
\hline$\gamma$ & Homogenous growth & Homogenous growth & Homogenous growth \\
\hline$\delta$ & ND & ND & ND \\
\hline$\varepsilon$ & $\begin{array}{l}\text { Small aggregates (less } \\
\text { than } 0.5 \mathrm{~mm} \text { ) }\end{array}$ & $\begin{array}{l}\text { Predominantly } \\
\text { homogenous growth }\end{array}$ & $\begin{array}{l}\text { Predominantly } \\
\text { homogenous growth }\end{array}$ \\
\hline
\end{tabular}

Table 4.2. Appearance of growth of the different classes of mutants in liquid CDM II $1 \%$ glucose. ND, not determined. 
In order to screen the mutant bank for stationary phase and carbon starvation survival mutants, the 898 mutants were cultured for up to three weeks in microtitre plates containing CDM II $1 \%$ glucose and CDM II $0.1 \%$ glucose at $27^{\circ} \mathrm{C}$, following an adaptation of the protocol used by Uhde et al. (1997) (see Chapter II). The reasoning was that comparison of the growth patterns of $R$. erythropolis SQ1 mutants in CDM II $1 \%$ glucose and $0.1 \%$ glucose would reveal mutants that, for example, could survive stationary phase after growth in low-carbon medium but not high-carbon, and vice-versa. Following 1, 8, 14 and 22 days of incubation in the microtitre plates, a replica of each plate was made on LB agar and incubated at $27^{\circ} \mathrm{C}$. After $48 \mathrm{~h}$ incubation (for the 1, 8 and 14 day samples) or $96 \mathrm{~h}$ incubation (for the 22 day samples) the mutants were classified according to the system described in Table 4.1 and the results recorded. Growth on plates was observed after 4 days for 22 days samples because growth occurred more slowly at that time point, as judged by observation of the wild-type growth in well A1 of each plate. A summary of the results is presented in Table 4.3.

It is interesting to note that in CDM $1 \%$ glucose, the number of class $\delta$ mutants remained almost constant, whereas the mutant classes $\beta, \varepsilon$ and $\gamma$ increased over time. This is particularly so for $\gamma$ mutants, increasing from 28 at $24 \mathrm{~h}$ to 58 at 14 days. Similar observations were made for CDM $0.1 \%$ glucose; the number of $\gamma$ mutants increased from 3 to 54 from day 1 to 14 . In addition, the $\delta$ mutants increased in number from 8 to 27. Four pigmentation mutants were also observed. Three were white and one was orange, instead of the salmon colour of $R$. erythropolis SQ1.

Nine mutants consistently failed to form colonies on LB agar, in contrast with the mutants above that failed to grow on agar only transitorily. An attempt to culture them 
in LB broth failed. They presumably have an insertion in a biosynthetic pathway, the product of which is not provided by LB or CDM. 


\begin{tabular}{|c|c|c|c|c|c|}
\hline \multirow{2}{*}{$\begin{array}{l}\text { Growth } \\
\text { medium }\end{array}$} & \multirow{2}{*}{$\begin{array}{l}\text { Mutant } \\
\text { class }\end{array}$} & \multicolumn{4}{|c|}{ Incubation time } \\
\hline & & 1 day & 8 days & 14 days & 22 days* \\
\hline \multirow{6}{*}{$\begin{array}{l}\text { CDM } 1 \% \\
\text { glucose }\end{array}$} & $\alpha$ & 829 & 804 & 763 & 767 \\
\hline & $\beta$ & 0 & 22 & 26 & 29 \\
\hline & $\gamma$ & 28 & 40 & 58 & 53 \\
\hline & $\delta$ & 19 & 17 & 18 & 20 \\
\hline & $\varepsilon$ & 0 & 4 & 4 & 10 \\
\hline & No growth & 22 & 11 & 29 & 21 \\
\hline \multirow{6}{*}{$\begin{array}{l}\text { CDM } 0.1 \% \\
\text { glucose }\end{array}$} & $\alpha$ & 866 & 800 & 765 & 781 \\
\hline & $\beta$ & 0 & 27 & 27 & 29 \\
\hline & $\gamma$ & 3 & 42 & 54 & 51 \\
\hline & $\delta$ & 8 & 12 & 27 & 19 \\
\hline & $\varepsilon$ & 0 & 2 & 2 & 3 \\
\hline & No growth & 21 & 15 & 23 & 17 \\
\hline
\end{tabular}

Table 4.3. Summary of the results of the mutant bank screening experiment.

At each time point indicated, the mutants grown in microtitre plates were duplicated onto LB agar. After 2 days of growth (4 days for 22-day samples), each mutant was scored and the score recorded.

*Total number for this time point is 900 , this is due to mutants 4C7 and 9A3 being classified as $\gamma$ and $\varepsilon$ simultaneously, i.e. their growth is similar to $\gamma$ with in addition an altered pigmentation; 4C7 is orange whereas 9A3 is white. 
Sixteen mutants, exhibiting weak or no growth in at least one stage of screening, were selected for further study. Detailed results of the stationary phase/carbon starvation screening for these mutants are shown in Table 4.4. It was expected that stationary phase survival mutants would grow at day 1 and maybe day 8 , and then die out (see mutant 3F5). It was also expected that mutants that are deficient in glucose-sensing mechanisms, for example, would die quickly in CDM $0.1 \%$ glucose, since they would not adapt to the low-energy conditions. Auxotrophic mutants were not expected to grow in either 1 or $0.1 \%$ glucose medium. 


\begin{tabular}{|c|c|c|c|c|c|c|c|c|}
\hline \multirow{3}{*}{ Mutant } & \multicolumn{8}{|c|}{ Extent of growth at various time points } \\
\hline & \multicolumn{4}{|c|}{ CDM $1 \%$ glucose } & \multicolumn{4}{|c|}{ CDM 0.1\% glucose } \\
\hline & $1 \mathrm{~d}$ & $8 \mathrm{~d}$ & $14 \mathrm{~d}$ & $22 \mathrm{~d}$ & $1 \mathrm{~d}$ & $8 \mathrm{~d}$ & $14 \mathrm{~d}$ & $22 \mathrm{~d}$ \\
\hline 1B2 & WT & ++ & WT & ++ & WT & WT & + & WT \\
\hline $1 \mathrm{~B} 3$ & + & WT & + & + & WT & WT & + & + \\
\hline $1 \mathrm{H} 1$ & WT & WT & WT & WT & WT & WT & + & WT \\
\hline 3B4 & - & + & - & - & WT & WT & - & + \\
\hline $3 \mathrm{~B} 10$ & - & + & - & - & - & + & - & - \\
\hline $3 \mathrm{~F} 5$ & WT & + & - & - & + & + & - & + \\
\hline 4G6 & WT & ++ & ++ & WT & WT & WT & + & WT \\
\hline 4G11 & + & WT & - & + & WT & WT & + & + \\
\hline $5 \mathrm{C} 11$ & WT & WT & WT & WT & WT & WT & + & + \\
\hline $5 \mathrm{~F} 8$ & - & WT & + & - & + & - & + & - \\
\hline $6 \mathrm{~B} 2$ & WT & WT & WT & + & WT & + & + & + \\
\hline $6 \mathrm{E} 6$ & - & WT & - & + & WT & WT & - & + \\
\hline $8 B 7$ & + & WT & WT & WT & WT & + & + & + \\
\hline 10D3 & ++ & ++ & ++ & ++ & WT & WT & + & WT \\
\hline $10 \mathrm{E} 1$ & + & WT & + & WT & WT & WT & + & + \\
\hline $10 \mathrm{E} 4$ & + & + & + & + & + & + & + & + \\
\hline
\end{tabular}

\section{Table 4.4. Results of screening of selected mutants.}

Detailed results for 16 mutants, selected for further study, from the mutant bank stationary phase/carbon starvation screening experiment. Mutants were grown in microtitre plates and plated on LB agar. Growth was observed at 2 days after plating (4 days for 22-day samples). WT, wild-type growth; ++, intermediate growth; +, weak growth; -, no growth was observed. 


\subsection{Growth curves of selected mutants in chemically defined medium}

The growth of wild-type $R$. erythropolis SQ1 was compared with that of mutants. The 16 mutants described in Table 4.4 were further investigated by cultivation in glass flasks, as was described previously for wild-type $R$. erythropolis SQ1. Briefly, an inoculum of the mutant culture was taken from the stock microtitre plate (LB + kanamycin) and inoculated in LB broth (10 $\mathrm{ml}$ in a universal). After overnight growth, this culture was used to inoculate $100 \mathrm{ml} \mathrm{CDM} \mathrm{IIA} 1 \%$ and $0.1 \%$ glucose in a $500 \mathrm{ml}$ conical flask to $0.01 \mathrm{OD}_{600 \mathrm{~nm}}$. Samples were taken at regular intervals. The $\mathrm{OD}_{600 \mathrm{~nm}}$ and viable plate counts were measured at regular intervals. Viable plate counts were determined by serial dilution and plating on LB agar, as described previously. Four mutants, 4G6, 10D3, 1B2 and 1H1 were found to behave differently from the wild-type.

As can be seen from Figure 4.3, mutants 4G6 and 10D3 did not survive well during prolonged stationary phase. Mutant 4G6 grown in CDM IIA $1 \%$ glucose reached a maximum cell density of $6.7 \times 10^{9} \mathrm{CFU} / \mathrm{ml}$ at 3 days, with a subsequent steady decrease in culturability until day 27 , when cell counts of $7.6 \times 10^{5} \mathrm{CFU} / \mathrm{ml}$ were obtained (0.01\% survival compared to maximum) (Figure $4.3 \mathrm{a})$. No drop in culturability was observed for mutant 4G6 grown in CDM IIA $0.1 \%$ glucose, but the cell densities obtained were consistently lower than those of the wild-type $R$. erythropolis SQ1.

Mutant 10D3 grown in CDM IIA $1 \%$ glucose (Figure $4.3 \mathrm{~b}$ ) reached a maximum of $5.5 \times 10^{9} \mathrm{CFU} / \mathrm{ml}$ at 12 days, showing that it probably grows slower than wild-type (maximum of $6.3 \times 10^{9} \mathrm{CFU} / \mathrm{ml}$ at 4 days). The viable cell density then decreased to 
$5.1 \times 10^{6} \mathrm{CFU} / \mathrm{ml}(0.1 \%$ survival $)$ at 27 days. When grown in CDM IIA $0.1 \%$ glucose, mutant 10D3 appeared to behave as wild-type $R$. erythropolis SQ1.

In contrast, only a modest decrease in culturability could be observed for mutants 1B2 and $1 \mathrm{H} 1$ in comparison with wild-type $R$. erythropolis SQ1 when grown in CDM IIA $1 \%$ glucose (Figure 4.4). For mutant 1B2, culturability dropped to approximately $1 \times 10^{8} \mathrm{CFU} / \mathrm{ml}$ at 20 days then remained constant. For mutant $1 \mathrm{H} 1$, culturability remained similar to wild-type until 27 days, when it dropped to $1 \times 10^{8} \mathrm{CFU} / \mathrm{ml}$. No significant decrease in culturability was observed when the mutants were grown in CDM IIA $0.1 \%$ glucose. 
(a)

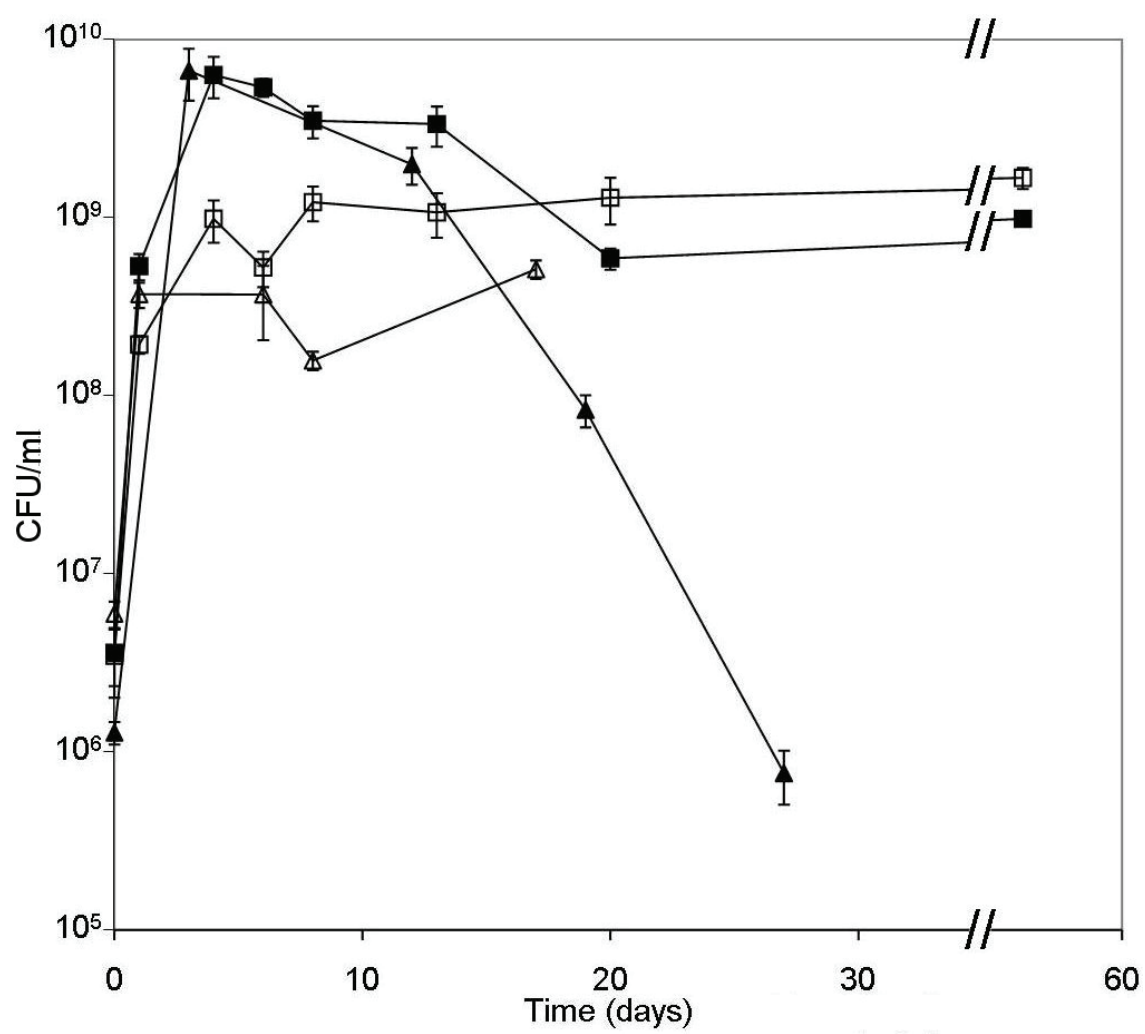

(b)

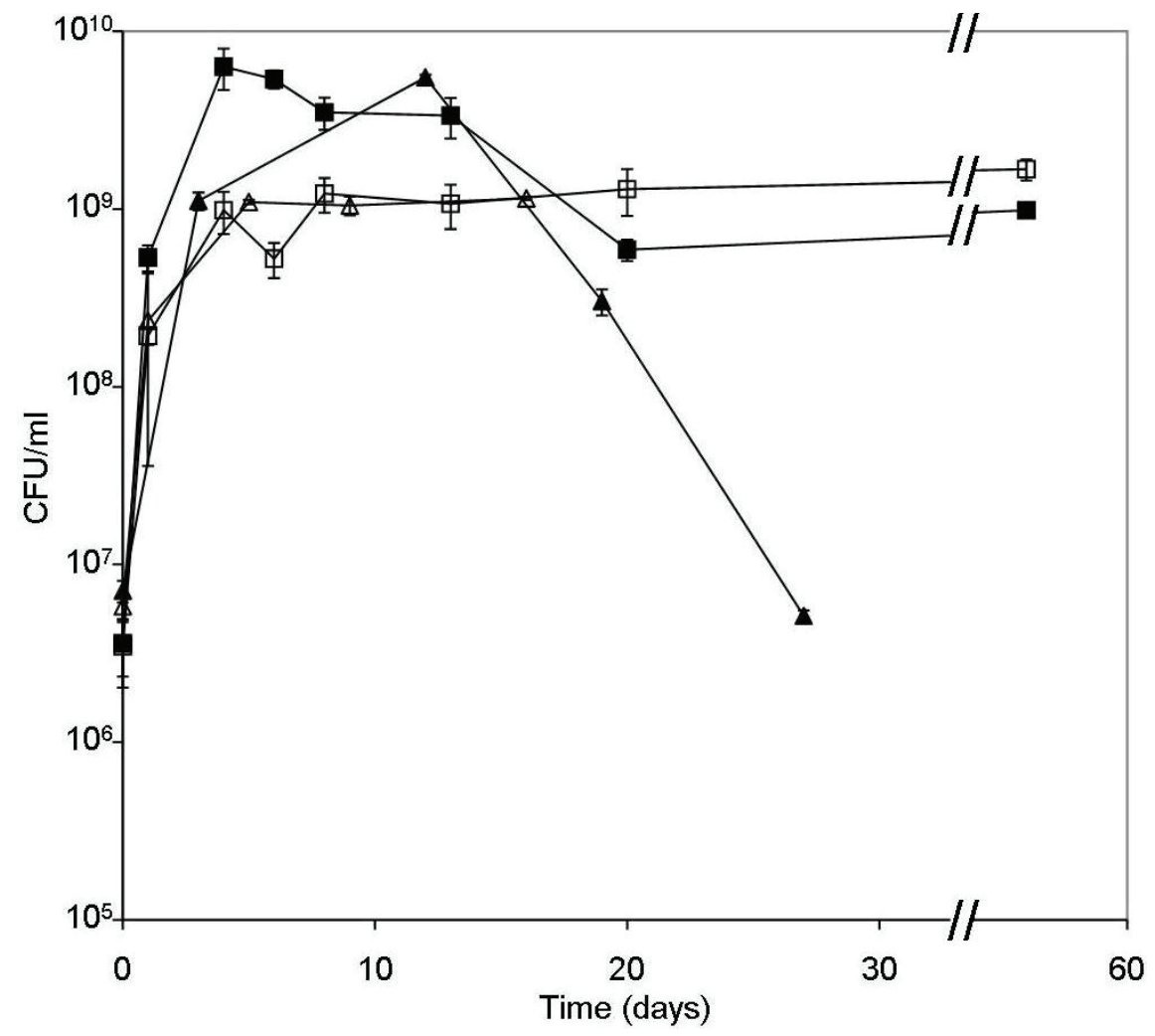

Figure 4.3. Growth curves of mutants 4G6 (a) and 10D3 (b) compared to wild-type $\boldsymbol{R}$. erythropolis SQ1. Data are from two separate experiments, plated in triplicate. Closed symbols, CDM IIA 1\% glucose; open symbols, CDM IIA 0.1\% glucose. - and $\square$, wild-type R. erythropolis SQ1; $\boldsymbol{\Delta}$ and $\triangle$, mutants 4G6 (a) and 10D3(b). 
(a)

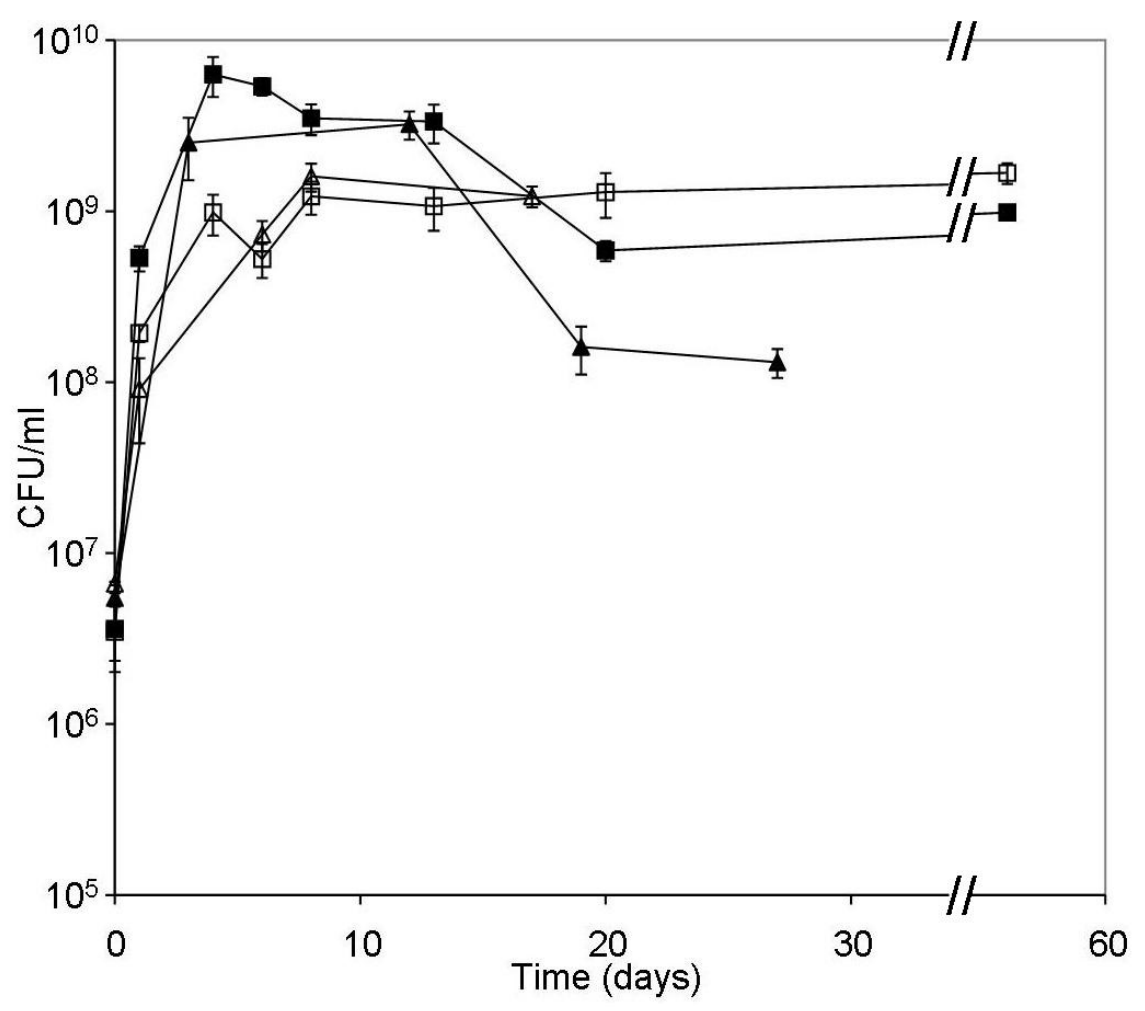

(b)

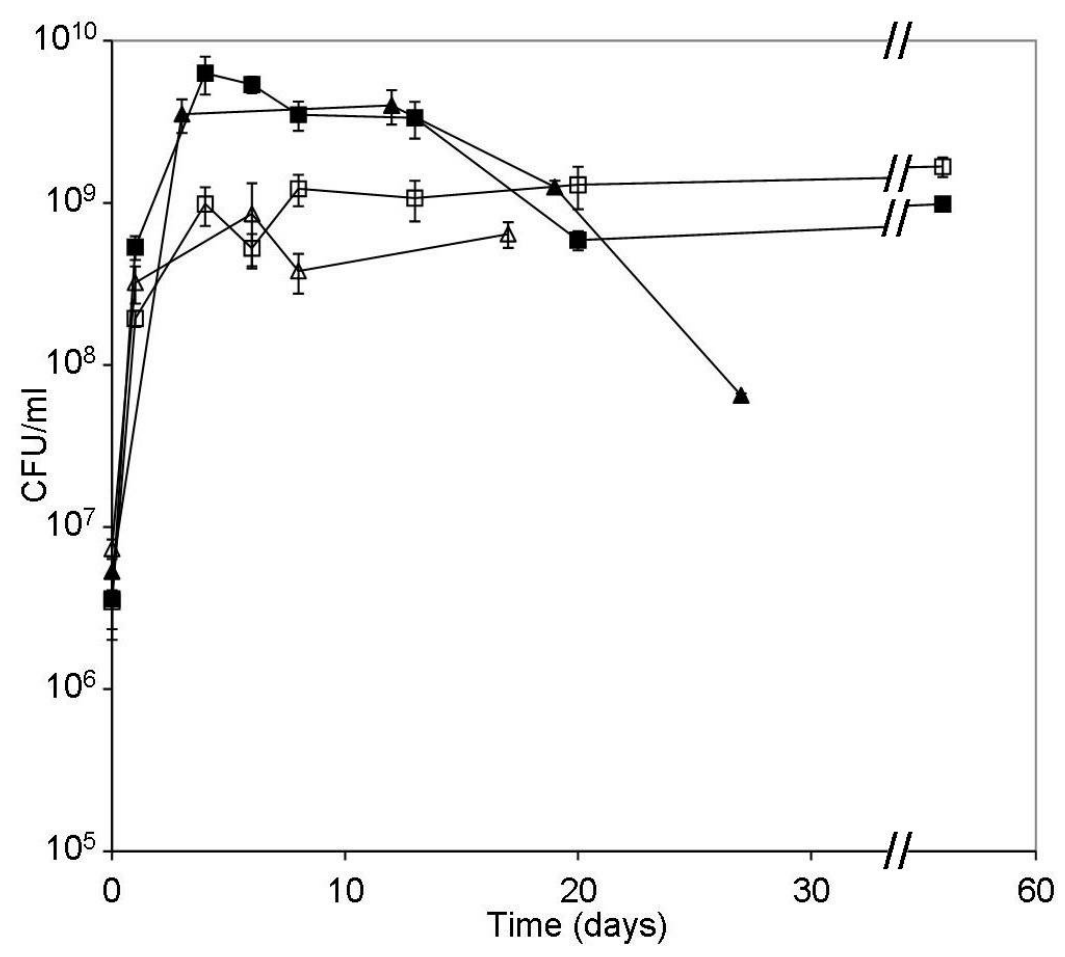

Figure 4.4. Growth curves of mutants $1 \mathrm{~B} 2$ (a) and $1 \mathrm{H1}$ (b) compared to wild-type R. erythropolis SQ1. Data are from two separate experiments, plated in triplicate. Closed symbols, CDM IIA $1 \%$ glucose; open symbols, CDM IIA $0.1 \%$ glucose. - and $\square$, wild-type R. erythropolis $\mathrm{SQ1}$; $\boldsymbol{\Lambda}$ and $\triangle$, mutants 1B2 (a) and 1H1(b). 


\subsection{Identification of genes and functions disrupted by transposon insertion}

The strategy used for obtaining the sequence of the DNA flanking the inserted transposon in any particular mutant was based on restriction and religation of the genomic DNA, followed by inverse PCR. Total genomic DNA was purified from the mutants as described in Chapter II and restricted using a panel of restriction endonucleases. The DNA from each restriction reaction was religated. A fraction of the religated DNA should carry the transposon. The DNA flanking the transposon was then amplified by inverse PCR using primers KAN-2_FP-1 and KAN-2_RP-1 (provided in the transposon kit, Epicentre Biotechnologies), that are specific to the $3^{\prime}$ and $5^{\prime}$ ends of the transposon, respectively, and designed to amplify DNA upstream and downstream of the kanamycin resistance gene (Figure 4.5). The amplicons were then cloned into pCR2.1 (Invitrogen) and sequenced using the M13 forward and reverse primers, specific to areas of the vector that are adjacent to the cloned sequence. 

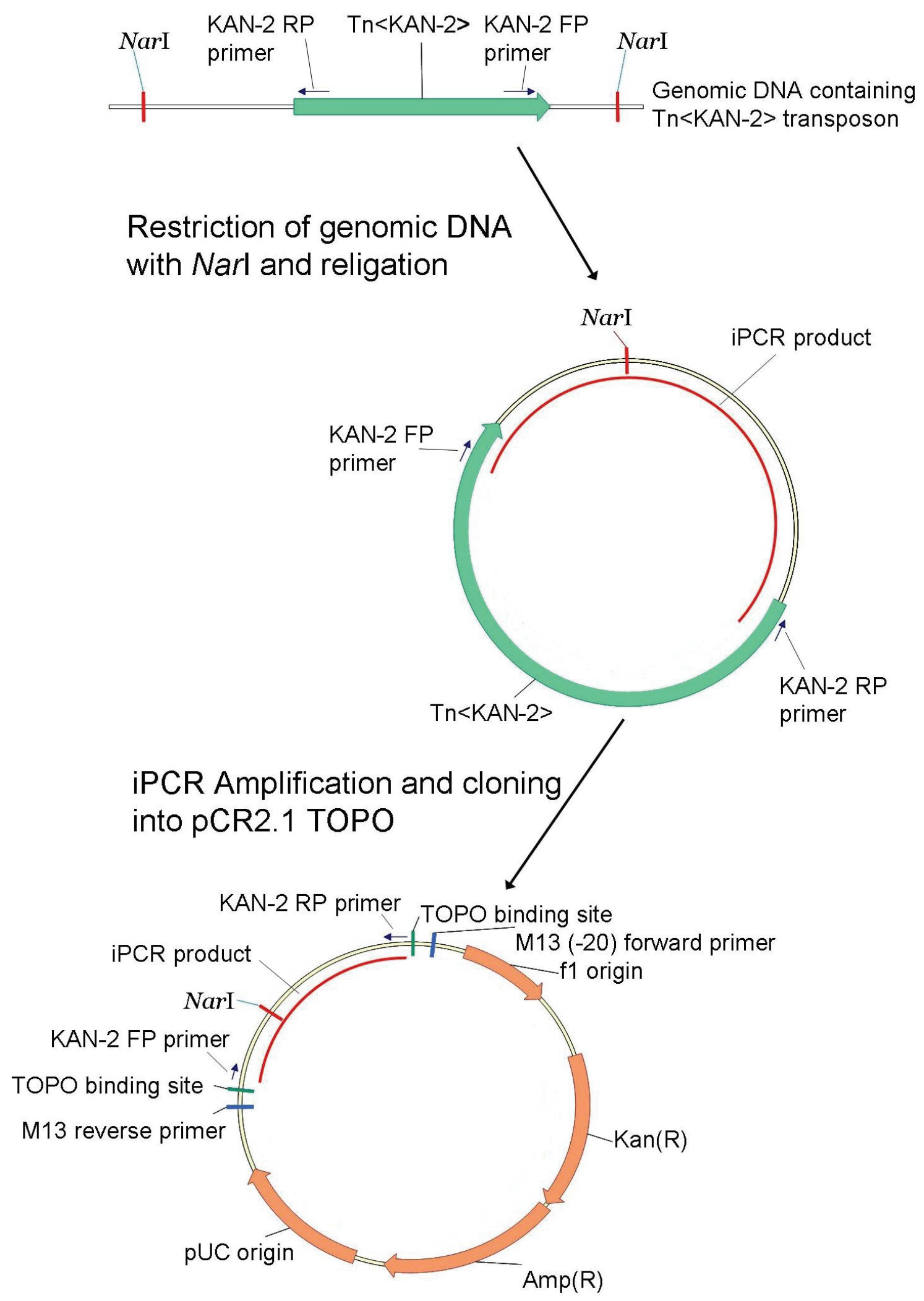

Figure 4.5. Principle of recovering DNA flanking a transposon insertion site by inverse PCR (iPCR). In this example NarI has been arbitrarily selected as the restriction endonuclease for restriction of the genomic DNA in the first step. 
Mutants selected by the screening strategy described above were grown in LB broth and their genomic DNA isolated for characterisation of the sequence disrupted by transposon insertion. Since it is not possible to predict which restriction endonuclease will yield a fragment that can be religated and amplified by iPCR, it is important in the first instance to restrict the genomic DNA using a range of restriction enzymes. For instance, using mutant $1 \mathrm{H} 1$ as an example, although EcoRI cuts the genomic DNA efficiently (Figure 4.6), iPCR products were only obtained for religated SalI-, BamHIand BclI-digested DNA. These products were of approximately $200 \mathrm{bp}, 1$ and $8 \mathrm{~kb}$, respectively (Figure 4.7). Therefore, for all the mutants investigated the first step of the analysis involved treating the genomic DNA with a range of restriction endonucleases, followed by religation and iPCR.

The iPCR amplification products of an appropriate size $(0.2-8 \mathrm{~kb})$ were then selected for cloning into the vector pCR2.1 using the TA cloning system (Invitrogen) and subsequent sequencing. The pCR2.1 vector is designed with binding sites for the primers M13 uni (-21) and M13 rev (-29) on either side of the cloning site, which can be used to verify the presence of inserted DNA by PCR and for sequencing the cloned DNA. 


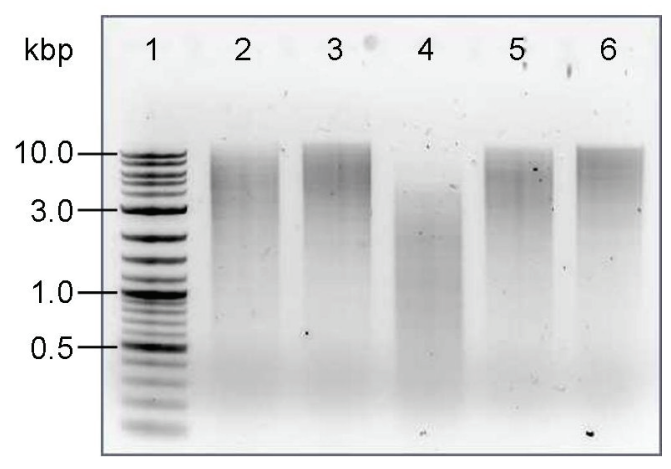

Figure 4.6. Total genomic DNA from mutant 1H1 subjected to restriction endonucleases and analysed by agarose gel electrophoresis. Lane 1, 2-Log DNA ladder (New England Biolabs); Lanes 2-6, DNA restricted by BclI, EcoRI, SalI, BamHI and $B g l$ II, respectively. Sizes of the molecular weight standards are shown on the left. 


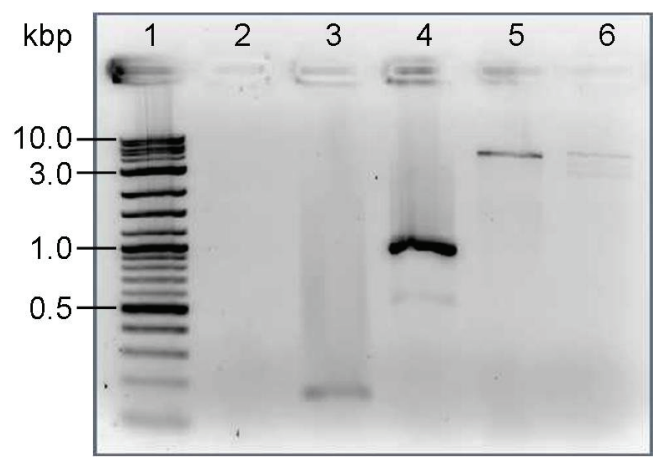

Figure 4.7. Agarose gel electrophoresis of DNA products of inverse PCR on religated DNA from mutant 1H1. Lane 1, 2-log DNA ladder; lanes 2-6, template was DNA restricted by EcoRI, SalI, BamHI, BclI and BglII, and religated prior to iPCR. Sizes of the molecular weight standards are shown on the left. 
For all mutants analysed, the DNA sequence of the cloned insert was determined using M13 uni (-21) and M13 rev (-29) primers. The resulting data was processed and analysed using the bioinformatic suite Vector NTI (Invitrogen) and online resources such as BLASTX and BLASTP at the NCBI (http://www.ncbi.nlm.nih.gov). The first step in analysing the products obtained by inverse PCR was to reconstruct the sequence by firstly identifying the restriction site for the endonuclease used to prepare the DNA template for iPCR and secondly by locating the transposon insertion site. Only the sequences between the restriction sites (if available) and the transposon insertion site were retained. The reverse complement of one of the two sequences was then generated and the two sequences amalgamated by joining at the transposon insertion site, thereby reconstructing the transposon insertion site and enabling analyses of the disrupted sequence. 


\subsubsection{Nucleotide excision repair mutant 4G6: uvrB mutant}

Genomic DNA from mutant 4G6 was isolated, restricted with a range of enzymes and then religated to perform iPCR. A $1.7 \mathrm{~kb}$ PCR amplification product was obtained from $B c l$ I-restricted template DNA, which was then cloned and sequenced as described earlier, yielding a sequence of $1671 \mathrm{bp}$. The sequence was examined for the presence of a 9 bp repeat sequence, a characteristic of Tn5 transposon insertion (Berg et al., 1983). In mutant 4G6 there is slight variation in the $9 \mathrm{bp}$ sequence upstream and downstream of the transposon. Alignment of the two sequences is thus:

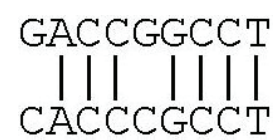

It can be seen that 2 out of $9 \mathrm{bp}$ are different, at positions 1 and 5 . Note that two guanines in the upstream sequence are replaced with two cytosines downstream. This did not result in any difference for the open reading frame search; the same ORFs were identified regardless of which of the $9 \mathrm{bp}$ sequence was retained in the reconstructed sequence (Figure 4.8). It should be noted that in all the other mutants analysed (described later in this chapter), the repeat sequences are identical.

Eight putative ORFs were identified in the 1671 bp sequence obtained (Figure 4.8). The ORFs were then analysed by comparison with the non-redundant protein database at NCBI using BLASTX. BLASTX translates the sequences in the six frames then searches the protein database against them; the bacterial genetic code was used for the translation. The most significant result $\left(E\right.$-values $\left.<1 \times 10^{-33}\right)$ were for ORF 8 and ORF 4 (Table 4.5). This was corroborated by performing a BLASTP search on all ORF frames. ORF 4 was found to be similar to an hypothetical protein from Rhodococcus sp. RHA1 and several Mycobacterium species. The protein predicted for ORF 8 was found to possess $73 \%$ amino acid identity to the 350 C-terminal amino acids of UvrB 
(excinuclease $\mathrm{ABC}$ subunit B) (Figure 4.9). UvrB is typically a protein of 673-721 amino acids (e.g. 673 and 698 amino acids for M. tuberculosis and E. coli, respectively) (Arikan et al., 1986; Darwin et al., 2003). It seems therefore that the sequence obtained for mutant $4 \mathrm{G} 6$ corresponds to the $3^{\prime}$ end of the $u v r B$ gene. 


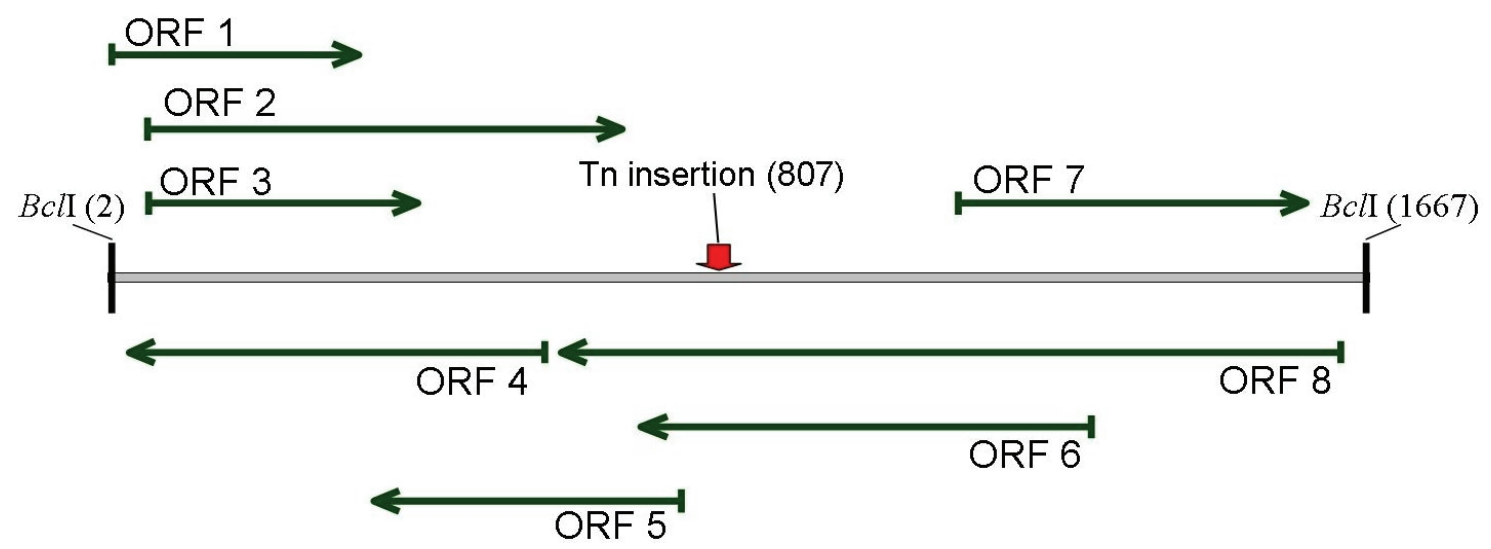

\begin{tabular}{|lccc|}
\hline Open reading frame & Position & Strand & Size \\
\hline ORF 1 & $3-329$ & Sense & $327 \mathrm{bp}$ \\
ORF 2 & $50-679$ & Sense & $630 \mathrm{bp}$ \\
ORF 3 & $52-408$ & Sense & $357 \mathrm{bp}$ \\
ORF 5 & $25-576$ & Antisense & $552 \mathrm{bp}$ \\
ORF 6 & $350-757$ & Antisense & $408 \mathrm{bp}$ \\
ORF 7 & $705-1301$ & Antisense & $597 \mathrm{bp}$ \\
ORF 8 & $1126-1587$ & Sense & $462 \mathrm{bp}$ \\
& $598-1632$ & Antisense & $1035 \mathrm{bp}$ \\
\hline
\end{tabular}

Figure 4.8. Schematic representation of the 1671 bp sequence retrieved for mutant 4G6. The $B c l$ r restriction sites used to retrieve the sequence are shown, as well as the transposon insertion site and putative open reading frames. Size, position and strand of the ORFs identified in the sequence are shown in the table.

ORFs were identified with the ORF Finder function of Vector NTI (Invitrogen, http://www.invitrogen.com). 


\begin{tabular}{|lll|}
\hline Organism & Protein & Score \\
\hline ORF 4 & & \\
Rhodococcus sp. RHA1 & Hypothetical protein RHA1_ro00975 & $6 \times 10^{-65}$ \\
Saccharopolyspora erythraea & Hypothetical protein SACE_2169 & $2 \times 10^{-43}$ \\
NRRL 2338 & & \\
Streptomyces coelicolor A3(2) & Hypothetical protein SCO6745 & $1 \times 10^{-34}$ \\
Mycobacterium sp. JLS & Hypothetical protein Mjls_2552 & $4 \times 10^{-33}$ \\
Mycobacterium sp. MCS & Hypothetical protein Mmcs_2515 & $4 \times 10^{-33}$ \\
ORF 8 & & \\
Rhodococcus sp. RHA1 & & $2 \times 10^{-161}$ \\
Mycobacterium gilvum PYR-GCK & Excinuclease ABC, B subunit & $8 \times 10^{-144}$ \\
Mycobacterium bovis BCG str. & Excinuclease ABC subunit B uvrB & $3 \times 10^{-143}$ \\
Pasteur 1173P2 & & $6 \times 10^{-143}$ \\
Nocardia farcinica IFM 10152 & Excinuclease ABC subunit B & \\
Mycobacterium tuberculosis & Excinuclease ABC subunit B & \\
CDC1551 & & \\
\hline
\end{tabular}

Table 4.5. BLASTX search results for ORF 4 and ORF 8. The genetic code was set to 11 (bacterial) and the search was performed on the non-redundant database. 
(1)

RHA 1

Nocardia

H3 $7 R v$

PYR-1

(1)

(1)

(1)

MVGNLPGFQLTEIVGEFVGTCAYPGCMAFASEHPVV-----AHSEFRPIGEIERSEARFEVVSDHKPAGD

PATETPAEGETPI AHSE 71 (1)

SQ1

RHA1

Nocardia

H3 $7 R v$

PYR-1

SQ1

RHA 1

Nocardia

H37Rv

PYR-1

SQ1

RHA1

Nocardia

H3 $7 R v$

PYR-1

(45)

(19)

QPAATADLERRINAGEKDVVLLGATGTGKSATTAWLIEKVORPTLVMMAPNKTLAAOLANELRDMLPNNS

(40)

14

(136) EYFVSYYDYYQPEAYIAQTDTYIEKDSS INDDVERLRHSATSSLLSRRDVVVVASVSCIYGLGTPQSYLD

(115) EYFVSYYDYYQPEAYIAQTDTYIEKDSS INDDVERLRHSATSSLLSRRDVVVVASVSCIYGLGTPQSYLD

(89) EYFVSYYDYYQPEAYIAQTDTYIEKDSS INDDVERLRHSATSALLSRRDVVVVASVSCIYGLGTPQSYLD EYFVSYYDYYQPEAYIAQTDTYIEKDSS INDDVERLRHSATSNLLSRRDVVVVASVSCIYGLGTPQSYMD 211

(1) ----

(206) RSVQLEVGVEVPRDALLRLLVDVQYTRNDLAFTRGSFRVRGDTVEI I PSYEELAVRIEFFGDEIEALYYI

(185) RSVQLEVGTEVDRDALLRLLVDVQYTRNDLSFTRGSFRVRGDTVEI IPSYEELAVRIEFFGDEIEALYYI

(159) RSVELKVGEEVPRDGLLRLLVDVQYTRNDMSFTRGSFRVRGDTVEI IPSYEELAVRIEFFGDEIEALYYI

(180) RSVELKVGDEVPRDGLLRLLVDVQYTRNDMAFTRGTFRVRGDTVEI IPSYEELAVRIEFFGDEIEELYYI 281

350

SQ1

RHA1

Nocardia

H37Rv

PYR-1

SQ1

RHA 1

Nocardia

H37Rv

PYR-1

SQ1

RHA1

Nocardia

H37Rv

(1)

(276) HPLTGDVVRKVDSVRIFPATHYVAGPERMERAVKDIEAELEERLADLEGKGKLLEAORLRMRTOYDLEM

(255) HPLTGDVVRKVDTLRIFPATHYVAGPDRMERAVRDIEQELEERLAELERQGKLLEAQRLRMRTQYDLEMI

(229) HPLTGEVIRQVDSLRIFPATHYVAGPERMAHAVSAIEEELAERLAELESQGKLLEAQRLRMRTNYDIEMM

(250) HPLTGDI IRKVDSLRIFPATHYVAGPERMAQA ISTIEAELEERLAELEGQGKLLEAQRLRMRTNYDIEMM 351

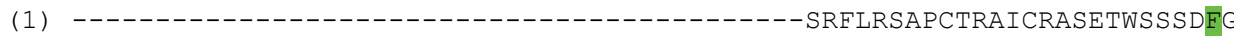

(346) KQVGFCSGIENYSRHIDGRGPGTAPATLIDYFPEDFLLVIDESHVTVPQI GAMYEGDMSRKRNLVEFGFR

(325) RQVGFCSGIENYSRHIDGRPAGSAPATLLDYFPEDFLLVIDESHVTVPQIGGMYEGDMSRKRNLVEYGFR

(299) RQVGFCSGIENYSRHIDGRGPGTP PATLLDYFPEDFLLVIDESHVTVPQI GGMYEGDISRKRNLVEYGFR

(320) RQVGFCSGIENYSRHIDGRPAGSAPATLLDYFPEDFLLVIDESHVTVPQIGGMYEGDMSRKRNLVDFGFR 421

(27) LPSATDNRPLTWEEFSQRIGQTVYLSATPGKYELGQSGGEFVEQVIRPTGLIDPEVIVKPTKGQIDDLVH

(416) LPSATDNRPLTWEEFTQRIGQTVYLSATPGKYELGQAGGEFVEQVIRPTGLVDPQVVVKPTKGQIDDLVH

(395) LPSAVDNRPLTWEEFADRIGQVVYLSATPGPYELGRTGGEVVEQVIRPTGLVDPKVVVKPTKGQIDDLVH

(369) LPSACDNRPLTWEEFADRIGQTVYLSATPGPYELSQTGGEFVEQVIRPTGLVDPKVVVKPTKGQIDDLIG

PYR-1

SQ1

RHA1

Nocardia

H37Rv

PYR-1

(390) LPSAVDNRPLTWEEFADRIGQTVYLSATPGSYELSQS GGEFVEQVIRPTGLVDPQVVVKPTKGQIDDLIG 491

(97) EIRERAERDERVLVTTLTKKMSEDLTDYLLELGIRVRYLHSDIDTLRRVELLRQLRLGEYDVLVGINLLR

(486) EIRERADRDERVLVTTLTKKMAEDLTDYLLELGIRVRYLHSDIDTLRRVELLRQLRLGEYDVLVGINLLR

( 465 ) EIRLRTERDERVLVTTLTKKMAEDLTDYLLGLGVRVRYLHSEIDTLRRVELLRQLRLGEYDVLVGINLLR

(439) EIRTRADADQRVLVTTLTKKMAEDLTDYLLEMGIRVRYLHSEVDTLRRVELLRQLRLGDYDVLVGINLLR

(460) EIRKRTERDERVLVTTLTKKMAEDLTDYLLEMGIRVRYLHSEVDTLRRVELLRQLRLGEYDVLVGINLLR 561

SQ1

RHA1

Nocardia

H37Rv

PYR-1

SQ1

RHA 1

Nocardia

H37Rv

PYR-1

SQ1

RHA1

Nocardia

H37Rv

(167) EGLDLPEVSLVAILDADKEGFLRSSTSLIQTIGRAARNVSGQVHMYADKITASMAQA IEETERRREKQVZ

(556) EGLDLPEVSLVAILDADKEGFLRSSTSLIQT IGRAARNVSGQVHMYADKITDSMQHA IEETERRREKQIA

(535) EGLDLPEVSLVAILDADKEGFLRSSTSLIQTIGRAARNVSGEVHMYADKITDSMQFAIEETERRRAKQIF

(509) EGLDLPEVSLVAILDADKEGFLRSSRSLIQTIGRAARNVSGEVHMYADKITDSMREAIDETERRRAKQIA

(530) EGLDLPEVSLVAILDADKEGFLRSPRSLIQT I GRAARNVSGEVHMYADKMTDSMKQAIDETERRRAKQTA 631

(237) YNEKMGVDPQPLRKKIADILDQVYEEAEDTAASVDVG--GSGRNATRGRRAQGEAGRAVSAGVYEGRDTK

(626) YNEKMGVDPQPLRKKIADILDQVYEEAEDTASGVDVG--GSGRNATRGRRAQGEAGRSVSAGVYEGRDTK

(605) YNEKMGIDPKPLRKKIADILDQVYREADEVEVGG------SGRNASRGRRAQGEPGRAVSAGVIEGRDVK

(579) YNEANGIDPQPLRKKIADILDQVYREADDTAVVE---VGGSGRNASRGRRAQGEPGRAVSAGVFEGRDTS

(600) YNKEHGIDPKPLRKKIADILDQVYREADDTEAAESVPIGGSGRNASRGRRAQGEPGRAVSAGVFEGRDTS 701

(305) SMPRAELADLVKELTGQMMNAARDLQFELAGRLRDEISDLKKELRGMDAAGLK

(694) SMPRAELADLVKELTNQMMNAARDLQFELAGRLRDEIADLKKELRGMDAAGLK

(669) SMPRAELADLVTELTAQMMNAARELQFELAGRLRDEIADLKKELRGMDAAGLS

(646) AMPRAELADLIKDLTAQMMAAARDLQFELAARFRDEIADLKRELRGMDAAGLK

PYR-1 (670) NMPRAELADLIKDLTAQMMAAARDLQFELAARIRDEIADLKKELRGMDAAGLK

Figure 4.9. Alignment of the putative UvrB sequence from $R$. erythropolis SQ1 with sequences from Rhodococcus sp. RHA1, Nocardia farcinica IFM 10152, M. tuberculosis H37Rv and M. vanbaalenii PYR-1. Grey background, >50 \% identity; green background, identical residues. 
The nucleotide excision repair pathway is ubiquitous in all organisms (Eisen \& Hanawalt, 1999), of which UvrB is an important subunit. UvrA and UvrB function, in E. coli, as a complex to identify conformational defects caused by DNA damage, by moving randomly along the DNA. The DNA conformation is changed by ATP hydrolysis and binding of UvrB to the DNA, with subsequent release of UvrA from the complex. UvrC will then attach to the DNA-UvrB complex and nick the DNA on either side of the lesion; the DNA-UvrB-UvrC complex is released by a helicase II. The gap is then filled by the DNA polymerase I (Lodish et al., 2000). M. tuberculosis uvrB transposon mutants were found to be hypersensitive to nitric oxide and UV light (Darwin et al., 2003). These UvrB mutants were also found to be less virulent in mice than wild-type strains (Darwin \& Nathan, 2005). Interestingly, M. tuberculosis uvrB mutants were shown to reach the same cell density as wild-type when grown in Middlebrook $7 \mathrm{H} 9$ broth supplemented with $0.2 \%$ glycerol, $0.05 \%$ Tween-80, $0.5 \%$ bovine serum albumin, $0.2 \%$ dextrose and $0.085 \%$ sodium chloride (Darwin et al., 2003). Although further work showed that $A B C$ excision repair has a role in the pathogenesis of $M$. tuberculosis (Darwin \& Nathan, 2005), and in particular in the resistance to nitric oxide, it is not known whether $u v r B$ mutants can survive long-term starvation.

In order to understand the genetic context of this transposon insertion in mutant 4G6, the arrangement of genes coding for the elements of the nucleotide excision repair pathway (uvrA-D) was investigated in Rhodococcus RHA1, M. tuberculosis CDC1551 and H37Rv, Nocardia farcinica IFM10152 and E. coli K12 using the genome visualisation program BacMap (Stothard et al., 2005), available at http://wishart.biology.ualberta.ca/BacMap. It should be noted that Rhodococcus RHA1 (McLeod et al., 2006) and R. equi 103 (www.sanger.ac.uk/Projects/R equi/) represent 
the only sequenced Rhodococcus genomes to date. It was found that in the genomes the $u v r$ genes are not organised in an operon, but rather are distributed across the genome of all these species. A putative ORF was identified downstream of $u v r B$ in R. erythropolis SQ1 that is very similar to ORF RHA1_ro00975 in Rhodococcus RHA1, but both are of unknown function. In other organisms a variety of genes are found downstream of $u v r B$, e.g. the molybdenum cofactor biosynthesis protein in E. coli $\mathrm{K} 12$ or the puromycin resistance protein in both $M$. tuberculosis CDC1551 and H37Rv. 


\subsubsection{Mutant with insertion downstream of putative IMP dehydrogenase gene guaB: mutant 10D3}

Mutant 10D3 was found to have a consistently weaker growth than wild type $R$. erythropolis SQ1 on all solid media tested (Section 4.1.2). Growth curves also showed that this mutant did not survive prolonged starvation in CDM IIA $1 \%$ glucose, with the culturability dropping to $0.1 \%$ of the maximum after 15 days in stationary phase. The genomic DNA was isolated and restricted with a panel of enzymes as previously described. iPCR yielded a $1.2 \mathrm{~kb}$ fragment from $B c l$ I-restricted genomic DNA, which was subsequently cloned and sequenced. A 1165 bp sequence was reconstructed and analysed for open reading frames (as described above), the results of which are shown in Figure 4.10.

When the nucleotide sequence was analysed against the non-redundant database at the NCBI using BLASTX, a putative inositol-5'-monophosphate dehydrogenase (GuaB)

was identified, encoded by ORF 4. Scores lower than $1 \times 10^{-100}$ were obtained with GuaB from Rhodococcus RHA1, N. farcinica IFM10152 and several Mycobacterium species. This result was confirmed by running the translated product of all the ORFs identified against the non-redundant database using BLASTP. The putative protein sequence obtained by translation of ORF 4 is 221 amino acids long and is highly similar to GuaB from various organisms. For example, amino acid identities of 93,88 and $85 \%$ were obtained with the GuaB of Rhodococcus RHA1, N. farcinica IFM10152 and $M$. tuberculosis H37Rv. The alignment of the ORF 4 amino acid sequence with selected IMP dehydrogenases of high similarity shows clearly that the sequence obtained for mutant 10D3 represents the $3^{\prime}$ end of a putative guaB gene. Alignment of the available putative $R$. erythropolis SQ1 IMP dehydrogenase sequence with the corresponding 
GuaB sequences returns an identity of $77 \%$ for the 200 C-terminal amino acids (Figure 4.11).

The inositol-5'-monophosphate dehydrogenase (EC 1.1.1.205) catalyses the dehydrogenation of inosine 5'-monophosphate into xanthosine 5'-phosphate (Figure 4.12). It is an essential step in the synthesis of guanosine 5'-phosphate (GMP). GMP then feeds directly into the synthesis of guanosine 5'-diphosphate (GDP), and from there into the synthesis of lipoarabinomannan, phosphatidylinositol mannosides and lipomannan (Yamazaki et al., 2006), and of course DNA. It is encoded by the gene guaB and is part of the guanine synthesis operon in E. coli (Vales et al., 1979). 


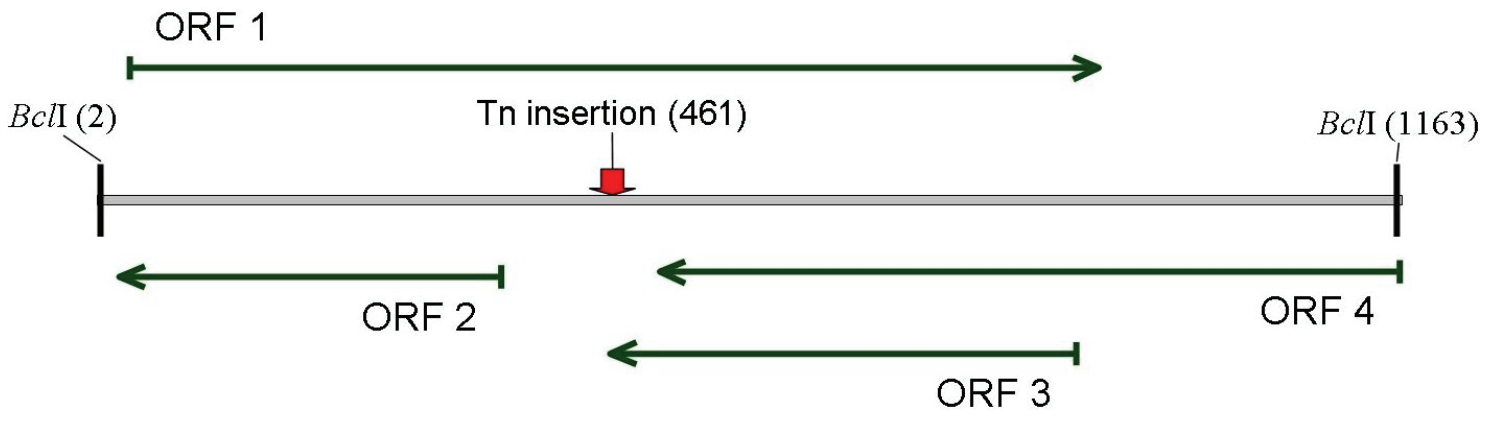

\begin{tabular}{|lcll|}
\hline Open reading frame & Position & Strand & Size \\
\hline ORF 1 & $29-895$ & Sense & $867 \mathrm{bp}$ \\
ORF 2 & $19-360$ & Antisense & $342 \mathrm{bp}$ \\
ORF 3 & $459-875$ & Antisense & $417 \mathrm{bp}$ \\
ORF 4 & $503-1165$ & Antisense & $663 \mathrm{bp}$ \\
\hline
\end{tabular}

Figure 4.10. Schematic representation of the $1165 \mathrm{bp}$ sequence retrieved for mutant 10D3. The BclI restriction sites used to retrieve the sequence are shown, as well as putative open reading frames predicted by ORF finder and the transposon insertion site. Size, position and strand of the ORFs identified in the sequence are shown in the table.

ORFs were identified with the ORF Finder function of Vector NTI (Invitrogen, http://www.invitrogen.com). 
SQ1

M. leprae

Mycobact

Nocardia

RHA1

SQ1

M. leprae

Mycobact

Nocardia

RHA 1

SQ1

M.leprae

Mycobact

Nocardia

RHA1

SQ1

M. leprae

Mycobact

Nocardia

RHA1

SQ1

M. leprae Mycobact

Nocardia

RHA1

SQ1

M.leprae Mycobact

Nocardia

RHA1

SQ1

M. leprae Mycobact

Nocardia

RHA 1

SQ1

M. leprae

Mycobact

Nocardia

RHA1

SQ1

M.leprae

Mycobact

Nocardia

RHA1
(1)

(1) MIRGMSNLKESSDFVASSYVRLGGLMDDPAATGGDNPHKVAMLGLTFDDVLLLPAASDVV

(1) MSRGMSGLEDSSDLVVS PYVRMGGLTTDPVPTGGDDPHKVAMLGLTFDDVLLLPAASDVV

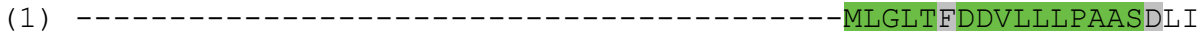

(1) --61

(1)

(61) PATADISSQLTKKIRLKVPLVSSAMDTVTEARMAIAMARAGGMGVLHRNLPVGEQAGQVE

(61) PATADTSSQLTKKIRLKVPLVSSAMDTVTESRMAIAMARAGGMGVLHRNLPVAEQAGQVE

(20) PSSVETSSRLTREIRLRTPLVSSAMDTVTEARMA IAMARAGGMGVLHRNLSAADQAAQVE

(39) PGQVDTSSQLTRDIRLRVPLVS SAMDTVTEARMA IAMARAGGMGVLHRNLSVEAQAGQVE 121

(1)

(121)

(121)

(80) TVKRSEAGMVTDPVTCRPTDTLAEVDAMCARFRISGLPVVDETGALVGI ITNRDMRFEVD

(99) TVKRSEAGMVTDPVTCKPSDTLAEVDAKCARFRISGLPVTDEAGQLVGI ITNRDMRFEVD 181

240

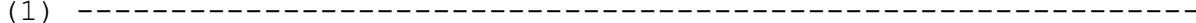

(181) QSKQVAEVMTKTPLITAAEGVSADAALGLLRRNKIEKLPVVDGHGRLTGLITVKDFVKTE

(181) QSKQVAEVMTKAPLITAQEGVSASAALGLLRRNKIEKLPVVDGRGRLTGLITVKDFVKTE

(140) QNRRVADVMTKAPLITAQEGVTAEAALGLLRRHKVEKLPIVDGNGRLRGLITVKDFVKTD

(159) QNRAVSEVMTKAPLITAQEGVTAEVALGLLRRHKIEKLPIVDGQGKLTGLITVKDFVKTE 241

300

(1)

(241)

(241)

QHPLATKDNDGRLLVGAAVGVGGDAWVRAMMLVDAGVDVLIVDTAHAHNRLVLDMVGKLK QHPLATKDS DGRLLVGAAVGVGGDAWVRAMMLVDAGVDVLVVDTAHAHNRLVLDMVGKLK QYPNATKDRDGRLLVGAAVGVGEDAWSRAMTLADAGVDVLVVDTAHGHQSQVLQMVAKVK QHPDATKDRDGRLLVGAAVGVGDEAWSRAMALTDAGVDVLVVDSAHGHSAGVLDMI SKLK 301

360

(1) -------I IGGNVATRAGALALVEAGVDAVKVGVGPGSICTTRVIAGVGAPQVTAILEA

(301) VEIGDRVQVIGGNVATRSAAAALVEAGADAVKVGVGPGSTCTTRVVAGVGAPQITAILEA

(301) SEVGDRVEVVGGNVATRSAAAALVDAGADAVKVGVGPGSICTTRVVAGVGAPQITAI LEA

(260) AEVGDRIQVVGGNIATRAGAAALVEAGADAVKVGVGPGSICTTRVVAGVGAPQITAILEA

(279) AEVDERVQI I GGNVATRSGAAALIEAGVDAVKVGVGPGSICTTRVIAGVGAPQITAILEA 361

420

(53) VAACRPLGVPVIADGGLQFSGDIAKALAAGASTAMLGSLLAGTAESPGELILVGGKQFKS

(361) VAACGPAGVPVIADGGLQYSGDIAKALAAGASTTMLGSLLAGTAEAPGEL IFVNGKQFKS

(361) VAACRPAGVPVIADGGLQYSGDIAKALAAGASTAMLGSLLAGTAEAPGELIFVNGKQYKS

(320) VAACKPAGVPVIADGGIQFSGDIAKAIAAGASTVMLGSLLAGTAESPGELILVGGKQFKS

(339) VAAAKPHGVPVIADGGLQFSGDIAKALAAGASTAMLGSLLAGTAESPGELILVNGKQYKS 421

480

(113) YRGMGSLGAMQSRGEAKSYSKDRYFQDDVLSEDKLVPEGIEGRVPFRGPLSQVTHQLTGG

(421) YRGMGSLGAMQGRGGDKSYSKDRYFADDALSEDKLVPEGIEGRVPFRGPLSSVI HQLVGG

(421) YRGMGSLGAMRGRGGATSYSKDRYFADDALSEDKLVPEGIEGRVPFRGPLSSVI HQLTGG

(380) YRGMGSLGAMQGRGQAKSFSKDRYFQDDVLAEDKLVPEGIEGRVPFRGPVNQVI HQLVGG

(399) YRGMGSLGAMQSRGAAKSYSKDRYFQDDVLSEDKLVPEGIEGRVAFRGPLSQVTHQLTGG 481

530

(173) LRAAMGYTGSATIEHLQNAQFVQITAAGLKESHPHDITMTVEAPNYTAR-

( 481 ) LRAAMGYTGSPTIEVLQQAQFVRITPAGLKESHPHDVAMTVEAPNYYPR-

(481) LRAAMGYTGSPTIEVLQQAQFVRITPAGLKESHPHDVAMTVEAPNYYAR-

(440) LRAAMGYTGSQSIADLQEAQFVQITAAGLKESHPHDITMTVEAPNYTGRS

(459) LRAAMGYTGASS IEELQNAQFVQITAAGLKESHPHDITMTVEAPNYTAR-

Figure 4.11. Alignment of a putative IMP dehydrogenase sequence from $R$. erythropolis SQ1 with sequences from Rhodococcus sp. RHA1 (guaB1), Nocardia farcinica IFM 10152 (guaB), and Mycobacterium leprae TN (guaB2).

Mycobact is the sequence of a IMP dehydrogenase found in M. tuberculosis H37Rv, M. tuberculosis CDC 1551 and M. bovis AF2122/97 (guaB2). Grey background, $>50 \%$ identity; green background, identical residues. 


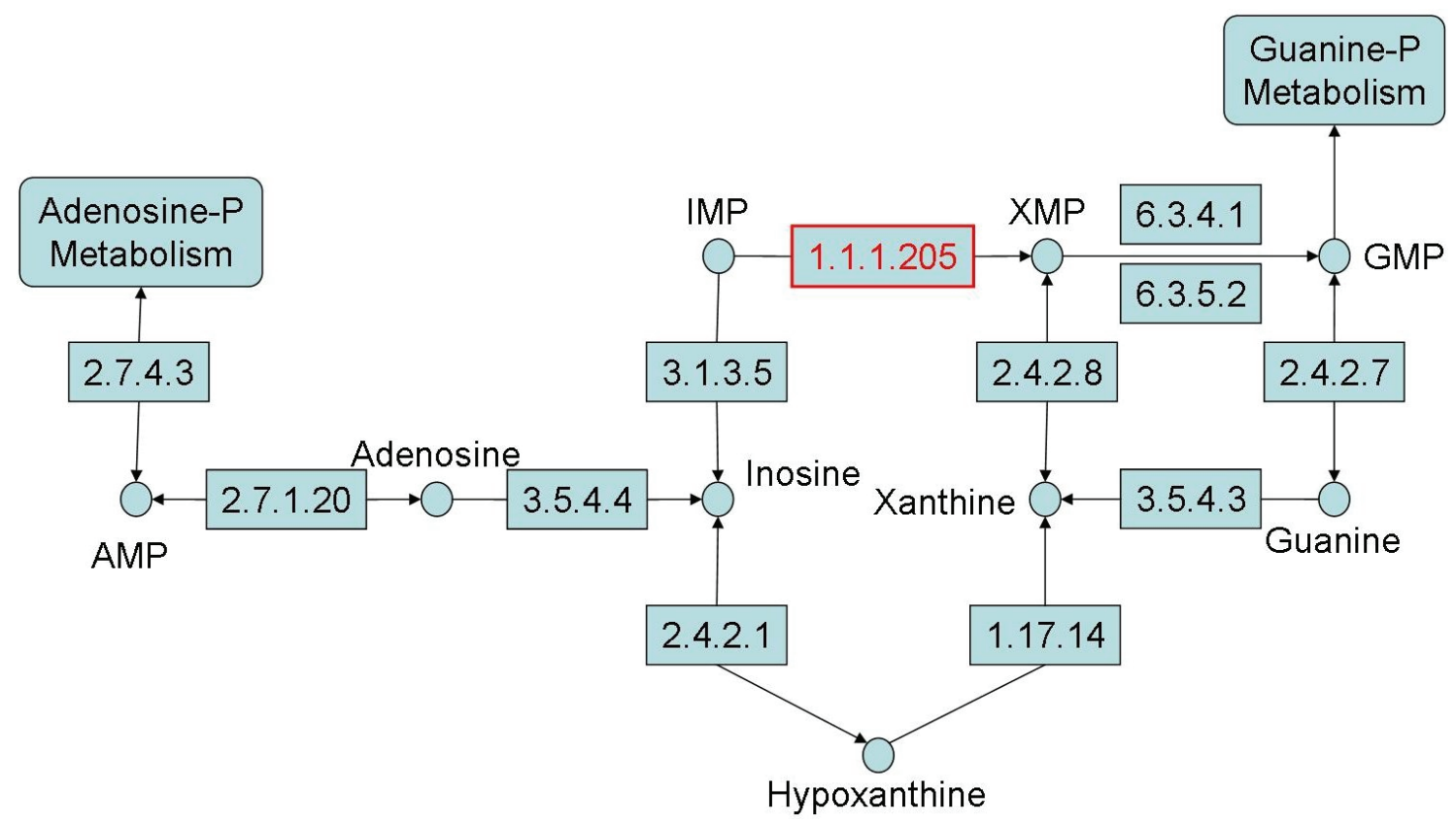

Figure 4.12. Position of the inositol-5'-monophosphate dehydrogenase in the purine metabolism pathway [adapted from the Rhodococcus sp. RHA1 pathway from KEGG (Kanehisa et al., 2006)]. 
It should be noted, however, that in mutant 10D3 the transposon did not insert into the putative guaB of $R$. erythropolis SQ1, but $42 \mathrm{bp}$ downstream of it. It is therefore unlikely that the function of the putative $g u a B$ is disrupted in this mutant. This was confirmed by growing mutant 10D3 in chemically defined medium lacking guanine. The mutant grew like the wild-type, and the addition of guanine to the medium $\left(100 \mu \mathrm{g} \mathrm{ml}^{-1}\right)$ did not have any noticeable effect on the growth (data not shown). However, it should be noted that the step catalysed by the IMP dehydrogenase can also easily be circumvented via the synthesis of hypoxanthine as shown in Figure 4.12.

Many actinobacteria possess several copies of the guaB gene. According to the KEGG gene database Corynebacterium glutamicum, M. tuberculosis, M. bovis and M. leprae have three, and Nocardia farcinica, Streptomyces coelicolor and Rhodococcus sp. RHA1 each contain at least two copies. The putative guaB of $R$. erythropolis SQ1 was found to be most similar to guaB1 of Rhodococcus RHA1 and guaB2 of M. tuberculosis CDC1551 and H37Rv. In these and other organisms the guaB genes appear to be part of an operon, as determined using the genome visualisation program BacMap (Stothard et al., 2005). In $N$. farcinica IFM 10152, Rhodococcus sp. RHA1 and M. tuberculosis H37Rv, the guaB (guaB1 for RHA1 and guaB2 for H37Rv) gene is upstream of a second putative IMP dehydrogenase gene (corresponding to the ro_06200 in Rhodococcus RHA1, guaB3 in M. tuberculosis H37Rv and nfa8960 in N. farcinica). These two genes are then followed by a putative cholesterol oxidase gene in Rhodococcus RHA1 and M. tuberculosis H37Rv, and by guaA (bifunctional GMP synthase/glutamine amidotransferase protein) in $N$. farcinica (see Figure 4.13). A closer examination of the sequence information obtained from mutant 10D3 revealed that, in R. erythropolis SQ1, ORF2 (located immediately downstream of the putative guaB, 
ORF4) possesses high similarity to the translated guaBl of Rhodococcus RHA1 with a score of $3 \times 10^{-38}$ when using BLASTX. 


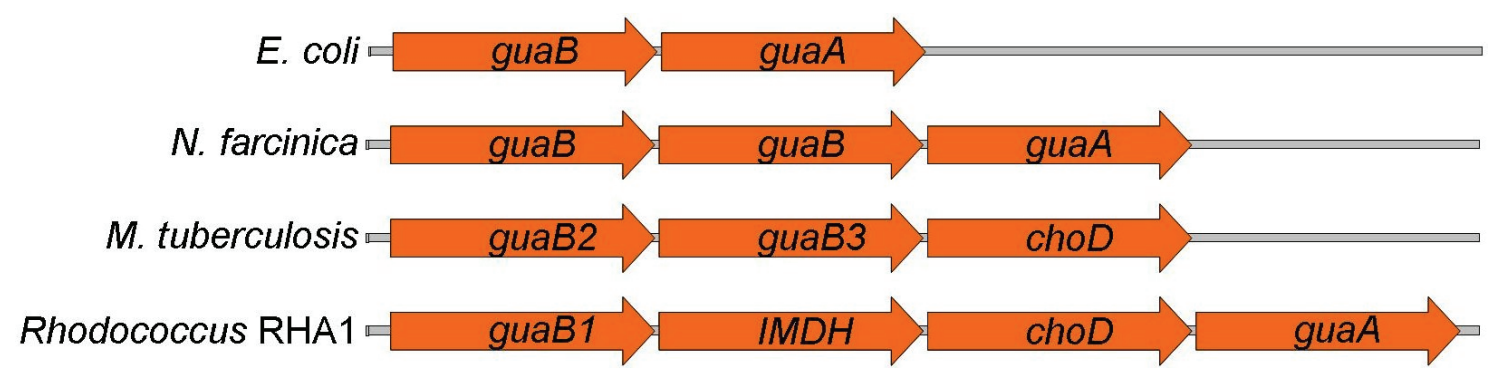

Figure 4.13. Schematic representation of the gua operon of $E$. coli K12, $N$. farcinica IFM 10152, M. tuberculosis H37Rv and Rhodococcus RHA1.

The putative guaB found in R. erythropolis SQ1 is most similar to guaB1, guaB2 and the first guaB in the $N$. farcinica operon. guaB and $I M D H$, IMP dehydrogenase; choD, cholesterol oxidase; guaA, GMP synthase. 


\subsubsection{Putative thioredoxin mutant: $1 \mathrm{H} 1$}

Mutant $1 \mathrm{H} 1$ failed to survive long-term starvation in flasks. When grown in flasks in CDM $0.1 \%$ glucose, mutant $1 \mathrm{H} 1$ was found to behave like the wild-type (Section 4.3). However, in CDM $1 \%$ glucose, the mutant behaved according to the wild-type until approximately 20 days into stationary phase, then the culturability dropped to $1.6 \%$ of the maximum at 27 days.

Genomic DNA of the mutant was isolated and restricted with a panel of enzymes and then subjected to religation and iPCR. A $1 \mathrm{~kb}$ amplicon was obtained when BamHIrestricted DNA was used as the DNA template for iPCR. This fragment was cloned and sequenced. For reasons that remain to be elucidated, sequence information could only be obtained for one side flanking the transposon insertion site. Hence, the sequence obtained was short $(844 \mathrm{bp})$ compared to that obtained for the other mutants $(>1200 \mathrm{bp})$. The details of the open reading frames identified are provided (Figure 4.15).

When the sequence was run against the non-redundant database at NCBI using BLASTX, strong similarity was found between the protein sequence encoded by ORF 1 and the sequence of an hypothetical protein of several actinomycetes, including Rhodococcus RHA1 (92\% amino acid identity), Nocardia farcinica (82\% identity), Mycobacterium ulcerans (69\% identity) and M. tuberculosis H37Rv (67\% identity). This protein was identified as a phosphoglycerate mutase in Saccharopolyspora erythraea, M. tuberculosis CDC1551 and Streptomyces avermitilis.

BLASTX identified the area corresponding to nucleotides $644-844$ as similar to several putative thioredoxins/redoxins in Rhodococcus RHA1 $\left(\right.$ E-score $\left.=5 \times 10^{-27}\right)$, Nocardia 
farcinica $\left(2 \times 10^{-19}\right)$ and several Mycobacterium species (minimum $2 \times 10^{-22}$, M. vanbaalenii PYR-1), as well as a putative cytochrome $c$ biogenesis protein in Nocardia farcinica IFM $10152\left(\right.$ E-score $\left.=2 \times 10^{-22}\right)$. It is not clear whether this sequence is part of an ORF, but it should be noted that the transposon insertion occurs at position 844 (Fig. 4.15) The ORF could potentially start at $575 \mathrm{bp}$, where an ATT triplet codes for isoleucine and is in-frame for coding of the putative thioredoxin. This would be an unusual start codon, ATT has been predicted to be start codon for four genes in E. coli (Makita et al., 2007), but has been demonstrated to be start codon only for infC and $p c n B$ (Butler et al., 1987; Jasiecki \& Wegrzyn, 2006). The only ATG codon in this area of the sequence is at $620 \mathrm{bp}$, but it is not in-frame for coding of the putative thioredoxin. Alignment and closer analysis of the sequence revealed that, although there is similarity to a segment of the N-terminal end of several thioredoxins, the most N-terminal residues have very low similarity to other thioredoxins (Figure 4.14). The putative thioredoxins identified above are very similar to DsbE from M. tuberculosis, which is involved in cytochrome maturation (Goulding et al., 2004). Interestingly, other genes involved in cytochrome production have been identified as important in starvation survival, such as cta in S. aureus (Clements et al., 1999b), and in resuming growth after stationary phase, such as $c y d C(\operatorname{sur} B)$ in E. coli (Siegele \& Kolter, 1993; Siegele et al., 1996). 
(---

RHA

(1)

作

Mgil (1) ----------------MNRVVAFGRT LMAACAATAVFAGCATGSDAVAQGG-TFEFV

Nfar1 (1) --------MRRPASFPAHRSAPVRVVAALLACVALVAGLAGCSTGTDAVATGG-TFEFV

Nfar2 (1) MTHLRTTKAARHGHRYGRARHRQLRLLAAMALVIAAVTAVISCASGSDSVATGGGTFDFV 61

SQ1 (39) SPGGQTKIFYDPPSDRGTIGKLSGPDLMNEGQKVGVDDFEGKVVVLNVWGQW--------

RHA1 (40) SPGGQTEIFYDPPADRGT IGTVSGPDLMTEGKTTSLDDFEGQVVVLNVWGQWCGPCRGEA

Mgil (41) APGGQTDIFYDPPQERGTPGPLSGPDLMDTDRTISLDDFAGKVVVINVWGQWCGPCRTEI

Nfar1 (51) SPGGKTDIFYDPPAARGTIGELSGPDLMTDGKTI SVADHPGQVVVLNIWGQWCGPCRAEA

Nfar2 (61) SPGGQTDIFYDPPESRGKIGVLAGPDLMAEDKTVSVSDYTGQVVVINLWGQWCGPCRAEA 121

NDLEQVYEETKDQGVSFLGINVRDNQQDKAQDFVIDNKVSYPS IYDPAMRTMIALGQNYP

Nfar2 (121) DDLERAYAATKASGVQFVGINVRDQQRDKAQDFVIDNKVSYPS IYDPPMRTLVALGGSYP $181 \quad 222$

SQ1 (91)

RHA1 (160) TSVIPTTIVLDREHRVAAVFLKELLAEDLKPVVERVAOES--

Mgil (161) TTVIPSTVVLDREHRVAAVFLRELLAQDLQPVVERLAAEQ--

Nfarl (171) TSVIPTTLVLDREHRVAAVFLRTLLAEDLQPVVQRIAEEGRQ

Nfar2 (181) TSVIPSTLILDRKQRVAAVFLRALLTSDLQPVIERLAAEQ--

Figure 4.14. Alignment of a putative thioredoxin from $R$. erythropolis SQ1 with sequences from Rhodococcus sp. RHA1, Mycobacterium gilvum and Nocardia farcinica IFM 10152.

SQ1, translation of sequence from 572-844 nt; RHA1, putative thioredoxin; Mgil, alkyl hydroperoxide reductase; Nfar1, putative cytochrome $c$ biogenesis protein; Nfar2, putative thioredoxin. All have a similar putative protein disulfide reductases (thioredoxin) function with important roles in cytochrome maturation.

Grey background, $>50 \%$ identity; green background, identical residues. 
No significant similarity could be found for ORF 2, whether by using BLASTX or BLASTP.

Phosphoglycerate mutase reversibly catalyses the transfer of a phosphate group from $\mathrm{C}_{2}$ to $\mathrm{C}_{3}$ of phosphoglycerate (Fothergill-Gilmore \& Watson, 2006). It is at the junction of glycolysis and neoglucogenesis, allowing the degradation of glucose into pyruvate and vice-versa. Phosphoglycerate mutases form a large family, many members of which have a wide range of substrates (e.g. phosphoglucomutase, involved in both glucose and galactose metabolism; (Berg et al., 2002). The gene coding for the putative phosphoglycerate mutase is upstream of a thioredoxin protein gene and a cytochrome $c$ biogenesis protein gene in the organisms with high similarity to ORF 1 of R. erythropolis SQ1. These genes are css $X$ and ccdA in Saccharopolyspora erythraea NRRL 2338, MUL_0625 and ccsA in Mycobacterium ulcerans Agy99, and Rv0526 and ccdA in M. tuberculosis H37Rv, respectively. It is not known whether these genes are organised in operons. 


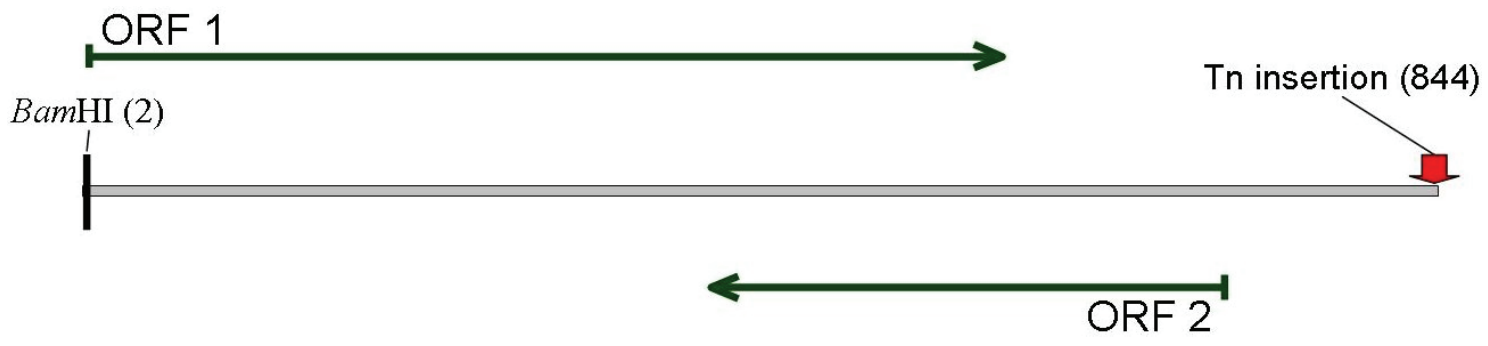

\begin{tabular}{|lccc|}
\hline Open reading frame & Position & Strand & Size \\
\hline ORF 1 & $3-572$ & Sense & $570 \mathrm{bp}$ \\
ORF 2 & $391-711$ & Antisense & $321 \mathrm{bp}$ \\
\hline
\end{tabular}

Figure 4.15. Schematic representation of the 844 bp sequence retrieved for mutant 1H1. The BamHI restriction site used to retrieve the sequence is shown, as well as putative open reading frames predicted by ORF finder and the transposon insertion site. Size, position and strand of the ORFs identified in the sequence are shown in the table. ORFs were identified with the ORF Finder function of Vector NTI (Invitrogen, http://www.invitrogen.com). 


\subsubsection{Histidine and Serine/Glycine auxotrophic mutants}

In addition to characterising potential starvation and/or stationary phase survival mutants, mutants that displayed different growth behaviour patterns than the $R$. erythropolis SQ1 parent strain were also investigated. These mutants were found to grow well on LB, but poorly on CDM IIA.

\subsubsection{Histidine auxotrophic his $A$ mutants: 1B3, 3B10, $4 \mathrm{G} 11$ and $10 \mathrm{E} 4$.}

\section{Nucleotide sequence analysis}

The cloned inverse PCR products were obtained from EcoRI-digested DNA for mutants 1B3 (1317 bp) and 4G11 (1267 bp), and from NarI-digested DNA for mutants 3B10 and 10E4 (both $1039 \mathrm{bp}$ ). Alignment of the four sequences obtained for these mutants showed that there was strong similarity between them. Using AlignX (part of Vector NTI) with standard settings (gap opening penalty $=15$; gap extension penalty $=6.66$ and gap separation penalty range $=8$ ), the nucleotide identity was calculated to be $99 \%$ for all four sequences. The identity variation was introduced by differences in the size of the fragments obtained, due to the use of different restriction enzymes in the initial stage of the analyses, i.e. EcoRI for mutants 1B3 and 4G11, and NarI for mutants 3B10 and 10E4 (Figure 4.16). 


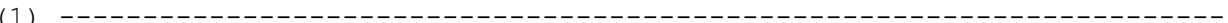

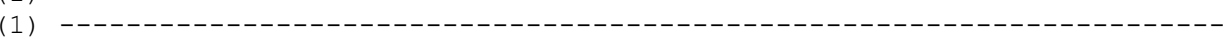

(1) GTCGAGAACGTGCGTTGTCGGCAACGCAGTTCCACCCCGAGAATCCGGTGACGCAGGCGCCGAGTTGCTG

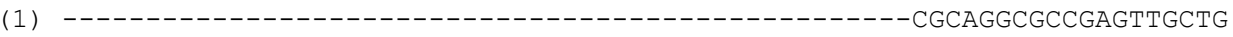

(1) GTCGAGAACGTGCGTTGTCGgCAACGCAGTTCCACCCCGAGAATCCGGTGACGCAGGCGCCGAGTTGCTG 71 140

(1) ---

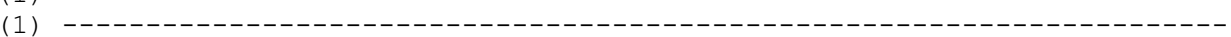

(71) AgCAACTGGGTGCAGAGTTTGTGACCGGGGGATCCGGACCGAACCGCGGTGACGATT-CTCCCTCGCCCG (20) AGCAACTGGGTGCAGAGTTTGTGACCGGGGGATCCGGACCGAACCGCGGTGACGATTTCTCCCTCGCCCG (71) AgCAACTGGGTGCAGAgTTTGTGACCGGGGGATCCGGACCGAACCGCGGTGACGATT CTCCCTCGCCCG 141 210

(1) --------------GGCGCCGCTGTGGCTGCGCTGGCGGTCGCAACCGTCGTCATCGTTGTTCTC (1) ------n---n------GGCGCCGCTGTGGCTGCGCTGGCGGTCGCAACCGTCGTCATCGTTGTTCTC (140) TCTGGCGTTGTTCTCGCTCGGCGCCGCTGTGGCTGCGCTGGCGGTCGCAACCGTCGTCATCGTTGTTCTC

(90) TCTGGCGTTGTTCTCGCTCGGCGCCGCTGTGGCTGCGCTGGCGGTCGCAACCGTCGTCATCGTTGTTCTC (141) tCTGGCGTTGTTCTCGCTCGGCGCCGCTGTGGCTGCGCTGGCGGTCGCAACCGTCGTCATCGTTGTTCTC 211

(52) AAACCATCGCCGGGCGTCGGCCTCGTGATCGGTCTCGTGGGGGTCGTCGCTGCGATTGCGGCAATGGGCG

(52) AAACCATCGCCGGGCGTCGGCCTCGTGATCGGTCTCGTGGGGGTCGTCGCTGCGATTGCGGCAATGGGCG

(210) AAACCATCGCCGGGCGTCGGCCTCGTGATCGGTCTCGTGGGGGTCGTCGCTGCGATTGCGGCAATGGGCG

(160) AAACCATCGCCGGGCGTCGGCCTCGTGATCGGTCTCGTGGGGGTCGTCGCTGCGATTGCGGCAATGGGCG

(211) AAACCATCGCCGGGCGTCGGCCTCGTGATCGGTCTCGTGGGGGTCGTCGCTGCGATTGCGGCAATGGGCG 281 350 (122) TCGTATCCAAGCGGATGACCCGAAAAGCGTTCGGTGACCCGGAAGACGGTTCGAGGTCCTGACCTACTAG (122) TCGTATCCAAGCGGATGACCCGAAAAGCGTTCGGTGACCCGGAAGACGGTTCGAGGTCCTGACCTACTAG (280) TCGTATCCAAGCGGATGACCCGAAAAGCGTTCGGTGACCCGGAAGACGGTTCGAGGTCCTGACCTACTAG (230) TCGTATCCAAGCGGATGACCCGAAAAGCGTTCGGTGACCCGGAAGACGGTTCGAGGTCCTGACCTACTAG (281) TCGTATCCAAGCGGATGACCCGAAAAGCGTTCGGTGACCCGGAAGACGGTTCGAGGTCCTGACCTACTAG 351 420 (192) GgTTACCGCACGTGAGCCTGGTCCTTTTGCCTGCTGTAGATGTCGTCAACGGTGAAGCTGTTCGCCTCGT (192) GGTTACCGCACGTGAGCCTGGTCCTTTTGCCTGCTGTAGATGTCGTCAACGGTGAAGCTGTTCGCCTCGT (350) GGTTACCGCACGTGAGCCTGGTCCTTTTGCCTGCTGTAGATGTCGTCAACGGTGAAGCTGTTCGCCTCGT (300) GGTTACCGCACGTGAGCCTGGTCCTTTTGCCTGCTGTAGATGTCGTCAACGGTGAAGCTGTTCGCCTCGT

(351) GGTTACCGCACGTGAGCCTGGTCCTTTTGCCTGCTGTAGATGTCGTCAACGGTGAAGCTGTTCGCCTCGT 421

(262) GCAAGGAGAGGCGGGAAGTGAGACCGGTTACGGGTCGCCCCGCGACGCTGCTCTTGCGTGGCAGAACGAC (262) GCAAGGAGAGGCGGGAAGTGAGACCGGTTACGGGTCGCCCCGCGACGCTGCTCTTGCGTGGCAGAACGAC (420) GCAAGGAGAGGCGGGAAGTGAGACCGGTTACGGGTCGCCCCGCGACGCTGCTCTTGCGTGGCAGAACGAC

(370) GCAAGGAGAGGCGGGAAGTGAGACCGGTTACGGGTCGCCCCGCGACGCTGCTCTTGCGTGGCAGAACGAC

(421) GCAAGGAGAGGCGGGAAGTGAGACCGGTTACGGGTCGCCCCGCGACGCTGCTCTTGCGTGGCAGAACGAC 491

(332) GGTGCCGAATGGGTGCATCTTGTCGACCTCGACGCTGCTTTCGGGCGTGGGTCCAACAGTGAACTGTTGG (332) GGTGCCGAATGGGTGCATCTTGTCGACCTCGACGCTGCTTTCGGGCGTGGGTCCAACAGTGAACTGTTGG (490) GGTGCCGAATGGGTGCATCTTGTCGACCTCGACGCTGCTTTCGGGCGTGGGTCCAACAGTGAACTGTTGG (440) GGTGCCGAATGGGTGCATCTTGTCGACCTCGACGCTGCTTTCGGGCGTGGGTCCAACAGTGAACTGTTGG (491) GGTGCCGAATGGGTGCATCTTGTCGACCTCGACGCTGCTTTCGGGCGTGGGTCCAACAGTGAACTGTTGG 561

(402) CCGGCGTCATCGGCGACCTGACGGTCAAGGTGGAACTGTCCGGTGGAATCCGCGACGACGCTTCCCTGGA (402) CCGGCGTCATCGGCGACCTGACGGTCAAGGTGGAACTGTCCGGTGGAATCCGCGACGACGCTTCCCTGGA (560) CCGGCGTCATCGGCGACCTGACGGTCAAGGTGGAACTGTCCGGTGGAATCCGCGACGACGCTTCCCTGGA (510) CCGGCGTCATCGGCGACCTGACGGTCAAGGTGGAACTGTCCGGTGGAATCCGCGACGACGCTTCCCTGGA

(561) CCGGCGTCATCGGCGACCTGACGGTCAAGGTGGAACTGTCCGGTGGAATCCGCGACGACGCTTCCCTGGA 631 700

(472) AGCCGCACTGGCCACCGGCTGTGCTCGCGTGAATCTCGGTACTGCCGCTATCGAGGATCCCGAGTGGTGT (472) AGCCGCACTGGCCACCGGCTGTGCTCGCGTGAATCTCGGTACTGCCGCTACCGAGGATCCCGAGTGGTGT (630) AGCCGCACTGGCCACCGGCTGTGCTCGCGTGAATCTCGGTACTGCCGCTATCGAGGATCCCGAGTGGTGT

(580) AGCCGCACTGGCCACCGGCTGTGCTCGCGTGAATCTCGGTACTGCCGCTATCGAGGATCCCGAGTGGTGT (631) AGCCGCACTGGCCACCGGCTGTGCTCGCGTGAATCTCGGTACTGCCGCTATCGAGGATCCCGAGTGGTGT 701 770 (542) GCGCGTGCATTGGCCAAGTACGGCGACAAGATCGCTGTCGGCCTCGATGTTCGTCTCGTCGACGGTCAGT (542) GCGCGTGCATTGGCCAAGTACGGCGACAAGATCGCTGTCGGCCTCGATGTTCGTCTCGTCGACGGTCAGT (700) GCGCGTGCATTGGCCAAGTACGGCGACAAGATCGCTGTCGGCCTCGATGTTCGTCTCGTCGACGGTCAGT (650) GCGCGTGCATTGGCCAAGTACGGCGACAAGATCGCTGTCGGCCTCGATGTTCGTCTCGTCGACGGTCAGT (701) GCGCGTGCATTGGCCAAGTACGGCGACAAGATCGCTGTCGGCCTCGATGTTCGTCTCGTCGACGGTCAGT 
10E4

(612) ACCGCACCCGTGGTCGCGGCTGGGTCACCGACGGCGGCGATCTGTGGGAGACCCTCGCGCGTCTGGACCG

(612) ACCGCACCCGTGGTCGCGGCTGGGTCACCGACGGCGGCGATCTGTGGGAGACCCTCGCGCGTCTGGACCG

(770) ACCGCACCCGTGGTCGCGGCTGGGTCACCGACGGCGGCGATCTGTGGGAGACCCTCGCGCGTCTGGACCG

(720) ACCGCACCCGTGGTCGCGGCTGGGTCACCGACGGCGGCGATCTGTGGGAGACCCTCGCGCGTCTGGACCG

(771) ACCGCACCCGTGGTCGCGGCTGGGTCACCGACGGCGGCGATCTGTGGGAGACCCTCGCGCGTCTGGACCG 841

(682) TGACGGCTGCACTCGTTACGTCGTCACCGACGTCTCCAAGGACGGCACACTCACCGGTCCCAATCTCGAA

(682) TGACGGCTGCACTCGTTACGTCGTCACCGACGTCTCCAAGGACGGCACACTCACCGGTCCCAATCTCGAA

(840) TGACGGCTGCACTCGTTACGTCGTCACCGACGTCTCCAAGGACGGCACACTCACCGGTCCCAATCTCGAA

(790) TGACGGCTGCACTCGTTACGTCGTCACCGACGTCTCCAAGGACGGCACACTCACCGGTCCCAATCTCGAA

(841) TGACGGCTGCACTCGTTACGTCGTCACCGACGTCTCCAAGGACGGCACACTCACCGGTCCCAATCTCGAA

(752) CTTCCCAGCCAGGTCTGCGCGGTCACCGACGCGCACGTCGTCGCCTCCGGTGGTGTGTCGACCATCGAGG

(752) CTTCCCAGCCAGGTCTGCGCGGTCACCGACGCGCACGTCGTCGCCTCCGGTGGTGTGTCGACCATCGAGG

(910) CTTCTCAGCCAGGTCTGCGCGGTCACCGACGCGCACGTCGTCGCCTCCGGTGGTGTGTCGACCATCGAGG

(860) CTTCCCAGCCAGGTCTGCGCGGTCACCGACGCGCACGTCGTCGCCTCCGGTGGTGTGTCGACCATCGAGG

(911) CTTCCCAGCCAGGTCTGCGCGGTCACCGACGCGCACGTCGTCGCCTCCGGTGGTGTGTCGACCATCGAGG 981

(822) ATCTACTCGCCATTTCCAGCCTGGTTGATCAGGGTGTCGAGGGCGCGATCGTGGGCAAGGCATTGTATGC

(822) ATCTACTCGCCATTTCCAGCCTGGTTGATCAGGGTGTCGAGGGCGCGATCGTGGGCAAGGCATTGTATGC

(980) ATCTACTCGCCATTTCCAGCCTGGTTGATCAGGGTGTCGAGGGTGCGATCGTGGGCAAGGCATTGTATGC

(930) ATCTACTCGCCATTTCCAGCCTGGTTGATCAGGGTGTCGAGGGCGCGATCGTGGGCAAGGCATTGTATGC

(981) ATCTACTCGCCATTTCCAGCCTGGTTGATCAGGGTGTCGAGGGCGCGATCGTGGGCAAGGCATTGTATGC

(892) CGGTCGCTTCACTCTCCCCGAAGCACTGGCAGCAGTTTCCGGATAGATTTTCCAGATGACTCCGACGCGT

(892) CGGTCGCTTCACTCTCCCCGAAGCACTGGCAGCAGTTTCCGGATAGATTTTCCAGATGACTCCGACGCGT

(1050) CGGTCGCTCCACTCTCCCCGAAGCACTGGCAGCAGTTTCCGGATAGATTTTCCAGATGACTCCGACGCGT

(1000) CGGTCGCTTCACTCTCCCCGAAGCACTGGCAGCAGTTTCCGGATAGATTTTCCAGATGACTCCGACGCGT

(1051) CGGTCGCTTCACTCTCCCCGAAGCACTGGCAGCAGTTTCCGGATAGATTTTCCAGATGACTCCGACGCGT

(962) GACCTCGATGAACTTCTCGCGATTGCGGGTCGGCTTCTCGACGGCGTCCACGATCAGTTCGTCTCCGGTG

(962) GACCTCGACGAACTTCTCGCGATTGCGGGTCGGCTTCTCGACGGCGTCCACGATCAGTTCGTCTCCGGTG

(1120) GACCTCGACGAACTTCTCGCGATTGCGGGTCGGCTTCTCGACGGCGTCCACGATCAGTTCGTCTCCGGTG

(1070) GACCTCGACGAACTTCTCGCGATTGCGGGTCGGCTTCTCGACGGCGTCCACGATCAGTTCGTCTCCGGTG

(1121) GACCTCGACGAACTTCTCGCGATTGCGGGTCGGCTTCTCGACGGCGTCCACGATCAGTTCGTCTCCGGTG

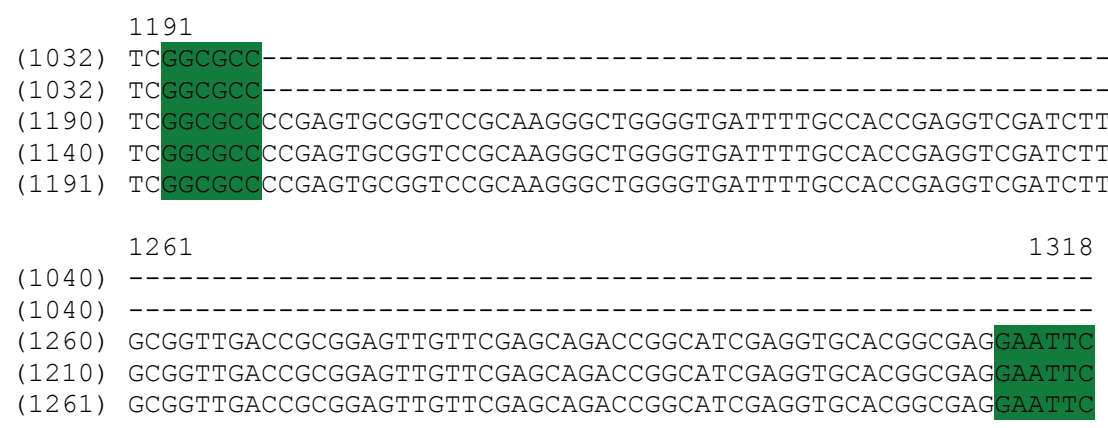

Figure 4.16. Alignment of the sequences obtained from mutants 1B3, 3B10, 4G11 and $10 \mathrm{E} 4$.

The transposon insertion site is highlighted in red and the $9 \mathrm{nt}$ sequence duplicated upon transposon insertion is in bold. The NarI and EcoRI restriction enzyme recognition sites are highlighted in green, and the nucleotides differing between sequences are in yellow (at positions 128, 680, 916, 1024, 1059 and 1129 using the consensus sequence as the reference). 
It is clear from the alignment that mutants 1B3, 3B10, 4G11 and 10E4 are clones, or that multiple mutants have been isolated in which the transposon insertion took place at the same site.

Open reading frames (ORF) were identified with the ORF Finder (part of Vector NTI). The default settings were used, with a minimum ORF size of 100 codons. Four ORFs were found - their relative positions and sizes are shown in Figure 4.17, together with the transposon insertion site and the enzyme restriction sites used to recover the sequences by iPCR. The consensus sequence was searched against the NCBI database using BLASTX. All the sequences identified as having high scores were obtained against the protein translated from sequence 383 to approximately $1100 \mathrm{bp}$ in frame +2 . This correlates with ORF 1 (Figure 4.16). The region immediately upstream of ORF 1 was analysed for transcription promoter sequences and is shown below:

\section{TGCGGCAATGGGCGTCGTATCCAAGCGGATGACCCGAAAAGCGTTCGGTG $-10+1$}

It should be noted that, while it not the most efficient or common start codon, GTG is encountered in many prokaryotes (Kozak, 1983). This is particularly true of high $\mathrm{G}+\mathrm{C}$ genome organisms, in particular in Mycobacterium (Rison et al., 2007). The putative transcription start is highlighted in red, and the -10 area in green. Although there is a poly-dA at -10 , it was not possible to find any -35 structure. 


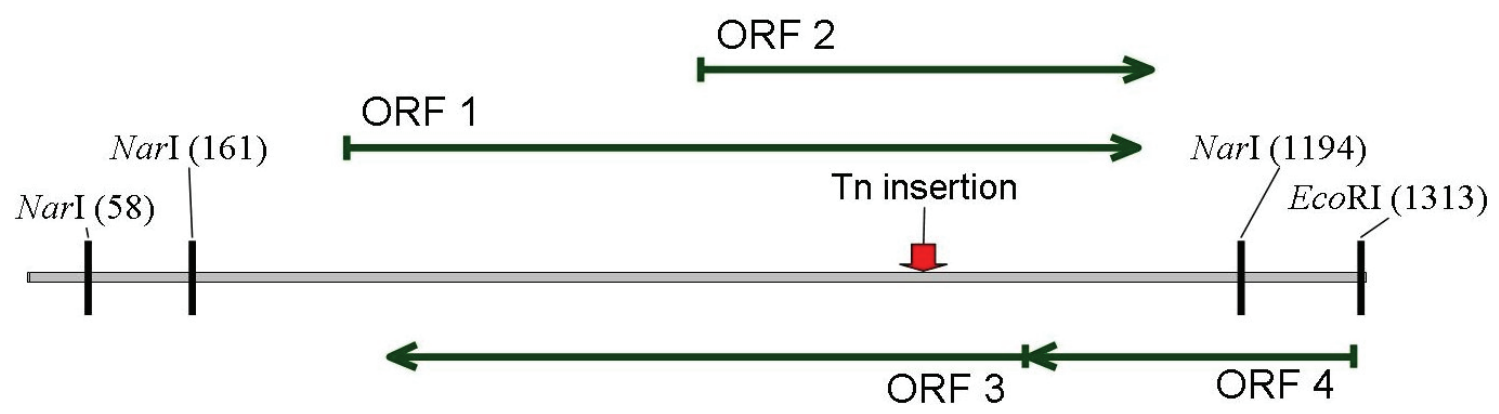

\begin{tabular}{|lccc|}
\hline Open reading frame & Position & Strand & Size \\
\hline ORF 1 & $313-1092$ & Sense & $780 \mathrm{bp}$ \\
ORF 2 & $662-1105$ & Sense & $444 \mathrm{bp}$ \\
ORF 3 & $355-981$ & Antisense & $627 \mathrm{bp}$ \\
ORF 4 & & & \\
& $985-1305$ & Antisense & $321 \mathrm{bp}$ \\
\hline
\end{tabular}

Figure 4.17. Schematic representation of the genomic area (1317 bp) disrupted in mutants 1B3, 3B10, 4G11 and 10E4.

The restriction enzymes used to retrieve the sequences are shown, as well as the transposon insertion site and putative open reading frames. Size, position and strand of the ORFs identified in the consensus sequence are shown in the table.

ORFs were identified with the ORF Finder function of Vector NTI (Invitrogen, http://www.invitrogen.com). 


\section{Translated ORF analysis}

All four open reading frames identified in the consensus sequence were translated using the bacterial genetic code, and then run against the non-redundant protein database at NCBI using BLASTP. The 260 amino acid protein obtained by translating ORF 1 was the only one yielding significant scores and $E$-values, confirming what had been determined with BLASTX. All results returned were either HisA or HisA/TrpF proteins; HisA is involved in histidine synthesis and His $\mathrm{A} / \operatorname{TrpF}$ in both histidine and tryptophan synthesis. Interestingly, both HisA and the bi-functional HisA/TrpF are the same size - 240 to 260 amino acids. The alignment of the putative HisA from R. erythropolis SQ1 with other HisA and HisA/TrpF sequences is shown in Figure 4.17.

There is experimental evidence for the activity of HisA from Streptomyces coelicolor A3, and HisA/TrpF of Mycobacterium tuberculosis H37Rv (Barona-Gómez \& Hodgson, 2003). HisA is a phosphoribosylformimino-5-aminoimidazole carboxamide ribonucleotide (ProFAR) isomerase (EC 5.3.1.16), catalysing the fourth enzymatic step between phosphoribosyl pyrophosphate (PRPP) and L-histidine (see Figure 4.19a, adapted from KEGG) in the histidine biosynthesis pathway. It mediates transformation of the phosphoribosyl-formimino-AICAR-phosphate (ProFAR) into phosphoribulosylformimino-AICAR-phosphate (PRFAR) via an Amadori rearrangement, consisting of the rearrangement of the aminoaldose ProFAR into the aminoketose, PRFAR. This also results in the opening of the ribose ring (illustrated in Figure 4.19b) (Henn-Sax et al., 2002). 
1

SQ1 (1) VTRKTVRGPDLLGLPHVSLVLLPAVDVVNGEAVRLVQGEAGSETGYGSPRDAALAWQNDG

RHA1 (1) --------------MSLVLLPAVDVVNGEAVRLVQGEAGSETGYGSPRDAALAWQNDG

NOC (1) --------------MSLVLLPAVDVANGEAVRLVQGEAGSETSYGSPRDAALAWQEAG

PYR (1) ---MTSVNP--SSSKPSALILLPAVDVVEGRAVRLVQGQAGSETEYGSALDAAMTWQRDG

MCS (1) ----MSVVPEKSVSEKRPLILLPAVDVVEGRAVRLVQGKAGSETEYGSALDAALGWQRDG 61

SQ1 (61) AEWVHLVDLDAAFGRGSNSELLAGVIGDLTVKVELSGGIRDDASLEAALATGCARVNLGT

RHA1 (45) AEWVHIVDLDAAFGRGSNRELLADVVGELDVQVELSGGIRDDASLEAALATGCGRVNLGT

NOC (45) AEWVHLVDLDAAFGRGSNRELLAKVVGELDVKVELSGGIRDDDSLEAALATGCARVNLGT

PYR-1 (56) AEWIHLVDLDAAFGRGSNRELLAEVVGKLDVAVELSGGIRDDDSLAAALATGCARVNLGT

MCS (57) AEWIHLVDLDAAFGRGSNRELLADVVGRLDVAVELSGGIRDDESLEAALATGCARVNIGT $\begin{array}{ll}121 & 180\end{array}$

SQ1 (121) AAIEDPEWCARALAKYGDKIAVGLDVRLVDGQYRTRGRGWVTDGGDLWETLARLDRDGCT

RHA1 (105) AAIENPEWCARAIAKYGEKIAVGLDVRLVDGEYQLRGRGWVTEGGNLWETLARLDKDGCS

NOC (105) AALEDPQWCARAIAKHGERIAVGLDVRI IDGDYRLRGRGWVSDGGDLWEVLERLERDGCS

PYR (116) AALENPQWCAKVVAEHGDKVAVGLDVKIVDGQHRLRGRGWETDGGDLWTVLDRLDGEGCS

MCS (117) AALENPQWCAKVVAEFGDKVAVGLDVKIVDDQHRLRGRGWETDGGDLWEVLDRLDSEGCS 181

SQ1 (181) RYVVTDVSKDGTLTGPNLELPSQVCAVTDAHVVASGGVSTIEDLLAISSLVDQGVEGAIV

RHA1 (165) RYVVTDVSKDGTLTGPNLELLAQVCAATDAPVVASGGVSTIDDLRAIAGLVDQGVEGSIV

NoC (165) RYVVTDVTKDGTLTGPNLELLSEVCAATEAPVIASGGVSAIEDLVAIAGLVPEGVEGAIV

PYR (176) RFVVTDVTKDGTLNGPNLELLTQVCERTDAPVIASGGVSSLDDLRAIATLTDRGVEGAIV

MCS (177) RYVVTDVTKDGTLQGPNLDLLGRVADRTDAPVIASGGVSSLDDLRAIATLTDRGVEGAIV 241

SQ1 (241) GKALYAGRFTLPEALAAVSG

RHA1 (225) GKALYAGRFTLPEALAAVSG

NOC (225) GKALYAGRFTLPEALAAVR-

PYR (236) GKALYAGRFTLPQALDAVGP

MCS (237) GKALYAGRFTLPEALAAMGQ

Figure 4.18. Alignment of a putative HisA sequence from $R$. erythropolis SQ1 with HisA sequences from Rhodococcus sp. RHA1 and Nocardia farcinica IFM 10152 (Noc), and with HisA/TrpF sequences from Mycobacterium vanbaalenii PYR-1 (PYR) and Mycobacterium sp. MCS.

Grey background, $>50 \%$ identity; green background, identical residues. 
(a)

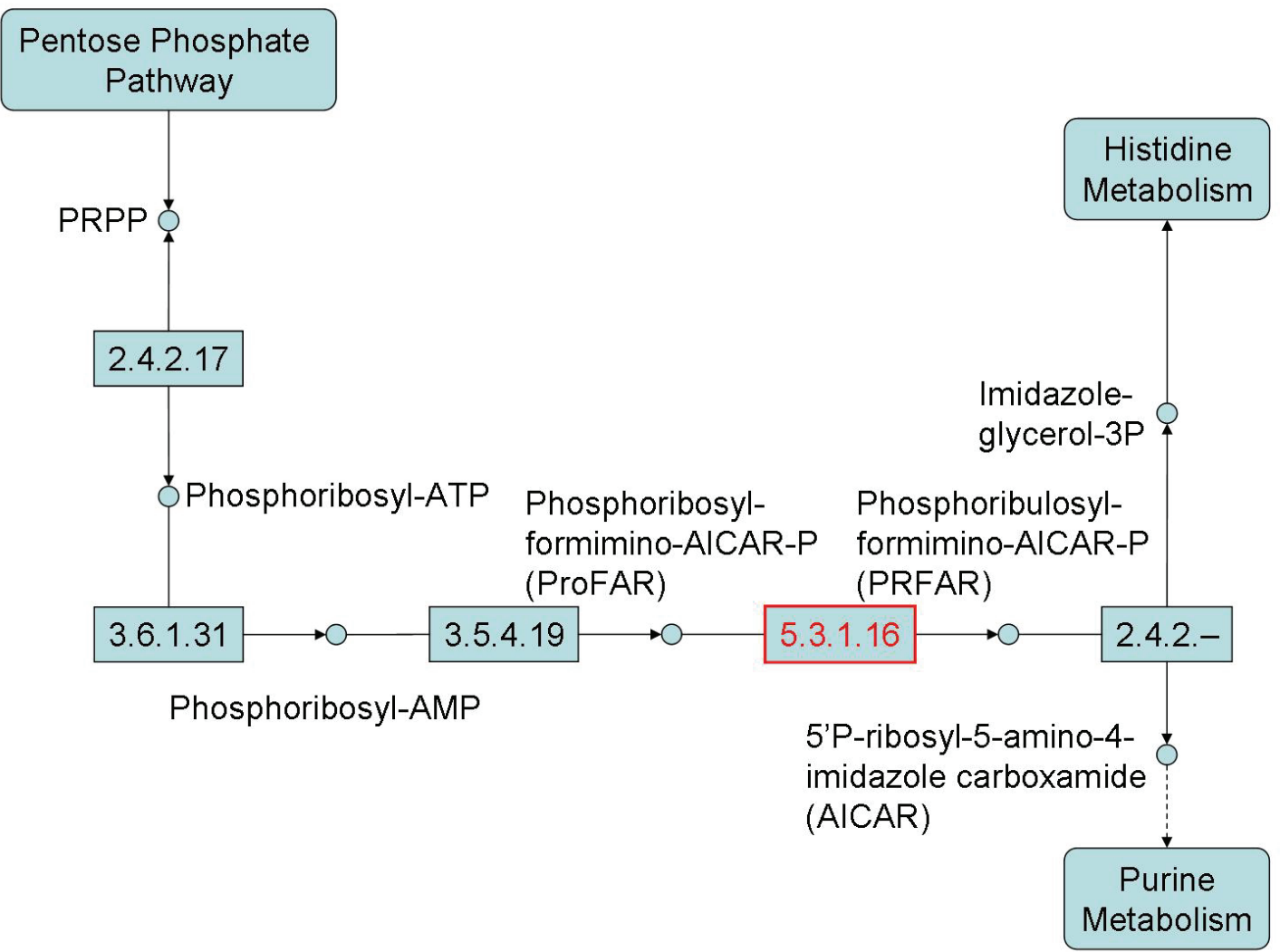

(b)

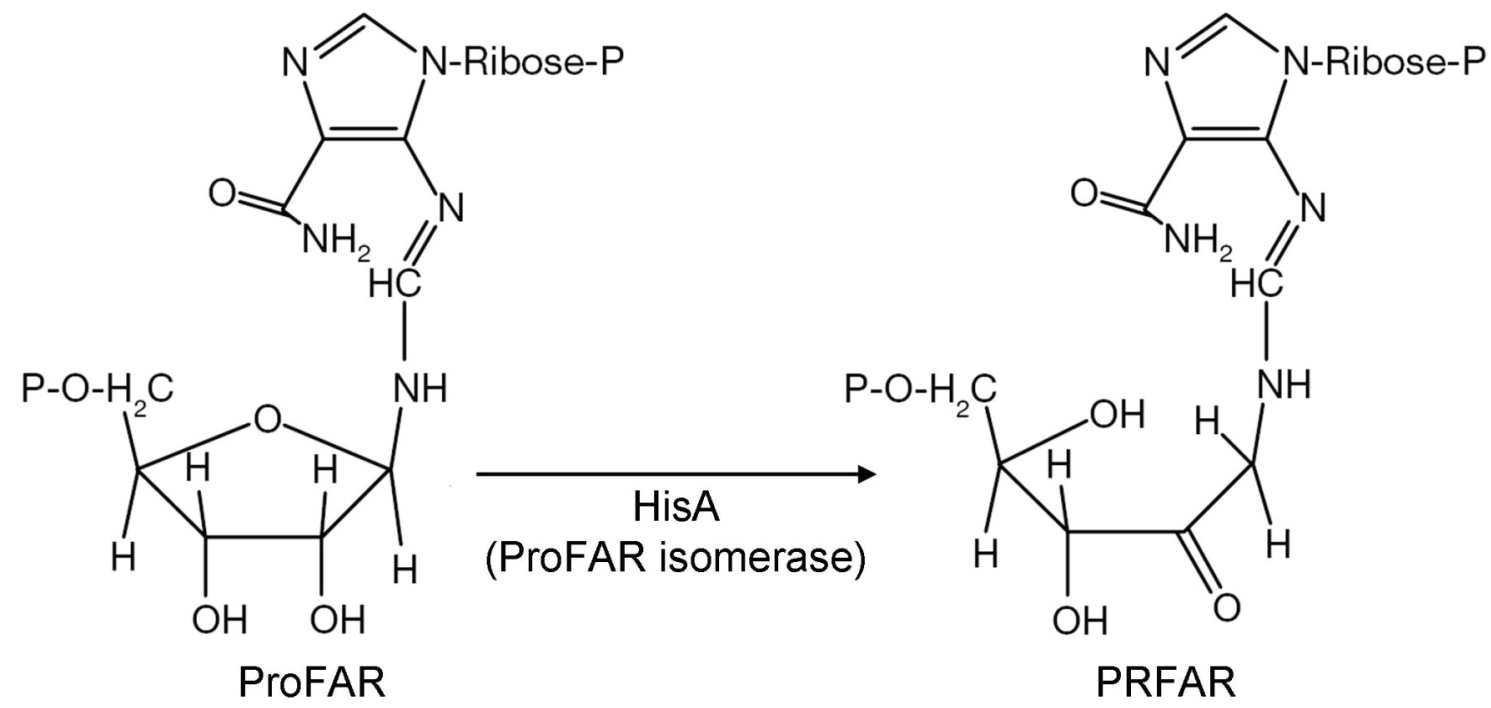

Figure 4.19. Function of HisA in the synthesis of histidine. (a) Position of His A (highlighted in red) in the histidine anabolic pathway [adapted from KEGG (Kanehisa et al., 2006)]. (b) Amadori rearrangement catalysed by HisA. A similar reaction is catalysed by TrpF. Adapted from Barona-Gómez and Hodgson (2003). 
This creates a link between the pentose phosphate pathway, purine metabolism and histidine metabolism. Interestingly, HisA is closely similar to the HisA/TrpF protein from Mycobacterium that is involved in both histidine and tryptophan synthesis. Both M. tuberculosis and Streptomyces coelicolor have a single enzyme to catalyse the Amadori rearrangement described in Figure $4.19 \mathrm{~b}$ in both the histidine and tryptophan biosynthesis pathways (Barona-Gómez \& Hodgson, 2003; Nester \& Montoya, 1976).

The data above suggests that mutants 1B3, 3B10, 4G11 and 10E4 could be auxotrophic for histidine, and possibly for tryptophan also. To verify this, an overnight LB culture of each mutant was washed twice in saline and inoculated in CDM II $1 \%$ glucose supplemented or not with histidine and/or tryptophan. Mutants 1B3, 3B10, 4G11 and 10E4 were shown to grow on $\mathrm{CDM}$ supplemented with histidine, but not on unsupplemented CDM or medium supplemented with tryptophan alone (data not shown). This would suggest that the putative gene disrupted by transposon mutagenesis does indeed code for HisA, and that this protein does not have any function similar to that of TrpF. 


\subsubsection{Histidine auxotrophic hisB mutant: $3 B 4$}

Similar to the mutants described in the previous section, mutant 3B4 was found to grow poorly on chemically defined media. This was most obvious on CDM IIA $1 \%$ glucose, where growth was observed only at day 8 (section 4.1.2). On CDM IIA $0.1 \%$ glucose, mutant 3B4 grew normally up to 8 days, then failed to produce growth at day 14 and produced only weak growth at day 22. Mutant 3B4 was therefore grown overnight in LB and its genomic DNA was isolated. It was then restricted with EcoR1, and inverse PCR was performed. A fragment of $2.4 \mathrm{~kb}$ was obtained, which was then cloned and sequenced, and the sequence analysed as described in Section 4.4. Comparison against the non-redundant database at the NCBI with BLASTX identified HisB, a putative imidazole-glycerol-phosphate dehydratase, encoded by an open reading frame of $611 \mathrm{bp}$. This sequence also corresponds to ORF 1, one of the open reading frames identified using ORF finder (Figure 4.20, Table 4.7).

This observation was confirmed when each ORF was translated using the bacterial genetic code and then analysed against the non-redundant protein database at the NCBI using BLASTP. The only translated ORF to yield significant scores and $E$-values was ORF 1 (lowest $E$-value $2 \times 10^{-96}$ ). The translated protein is 204 amino acids long and is highly similar ( $65.1 \%$ identity) to the HisB of other Corynebacterineae. The putative protein sequence is shown aligned with similar sequences in Figure 4.21. There is experimental evidence for this protein in N. farcinica IFM 10152 (accession no. Q5YYP8) (Ishikawa et al., 2004). The C-terminal part of HisB encodes an imidazoleglycerol-phosphate dehydratase function (Alifano et al., 1996), that dehydrates D-erythro-1-(imidazol-4-yl) glycerol 3-phosphate into 3-(imidazol-4-yl)-2oxopropyl phosphate, the sixth step in the biosynthesis of histidine from phosphoribosyl 
pyrophosphate (PRPP) (Figure 4.22). The N-terminal part of HisB has a phosphatase activity that transforms L-histidinol-P into L-histidinol (Alifano et al., 1996). 


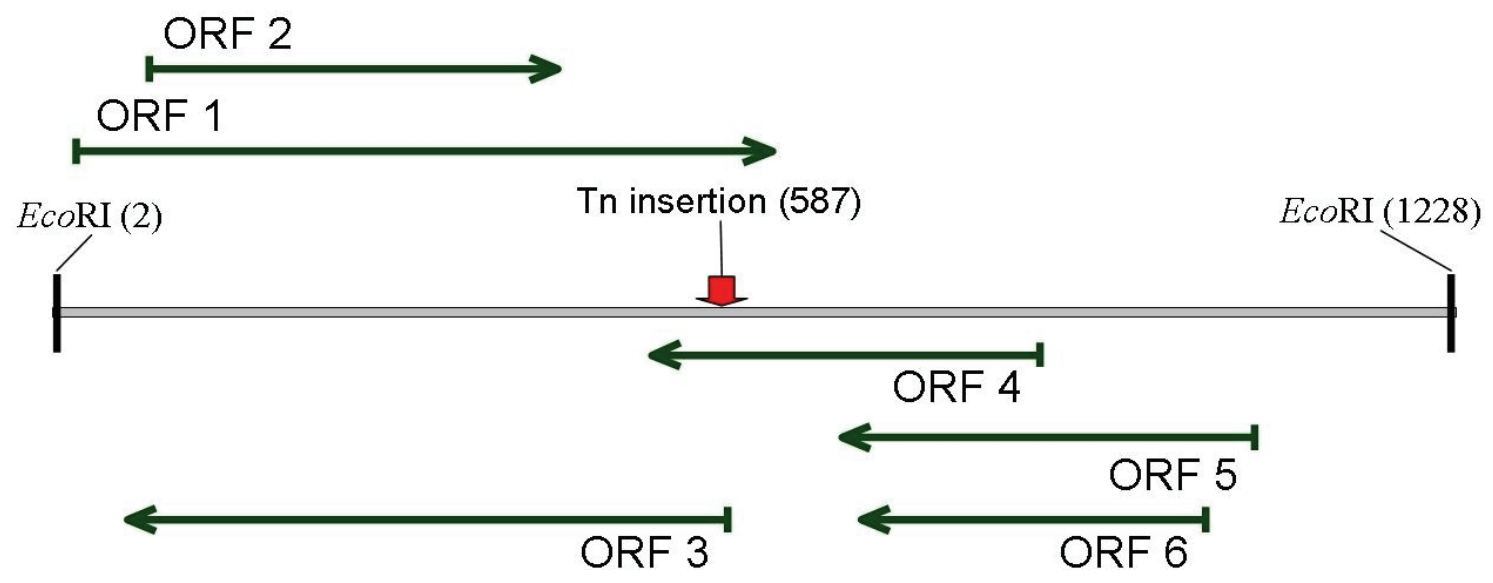

\begin{tabular}{|lccc|}
\hline Open reading frame & Position & Strand & Size \\
ORF 1 & $19-630$ & Sense & $612 \mathrm{bp}$ \\
ORF 2 & $83-442$ & Sense & $360 \mathrm{bp}$ \\
ORF 3 & $64-591$ & Antisense & $528 \mathrm{bp}$ \\
ORF 5 & $525-866$ & Antisense & $342 \mathrm{bp}$ \\
ORF 6 & $709-1011$ & Antisense & $303 \mathrm{bp}$ \\
& & & \\
\hline
\end{tabular}

Figure 4.20. Schematic representation of the $1232 \mathrm{bp}$ sequence retrieved from mutant 3B4.

The EcoR1 restriction sites used to retrieve the sequence are shown, as well as the open reading frames predicted by ORF finder and the transposon insertion site. Size, position and strand of the ORFs identified in the sequence are shown in the table.

ORFs were identified with the ORF Finder function of Vector NTI (Invitrogen, http://www.invitrogen.com). 
1

60

\begin{tabular}{|c|c|c|}
\hline SQ1 & (1) & -- -- - -MSDRIARIERTTRESS IVVELNLDGTGIVDVSTGVPFFDHMLNALGTHASFDL \\
\hline $\mathrm{K}-10$ & (1) & ---MTAVQAARRARIERRTKESDIVIELDLDGTGRVDVETGVPFYDHMLTALGSHASFDL \\
\hline H37Rv & (1) & MTTTQTAKASRRARIERRTRESDIVIELDLDGTGQVAVDTGVPFYDHMLTALGSHASFDL \\
\hline NoC & (1) & --MTDTTLRHRTARVERVTKESS IVVELDLDGTGRTDISTGVPFYDHMLTALGAHASFDI \\
\hline \multirow[t]{2}{*}{ RHA1 } & (1) & -- -- --MTDRIARVERTTKESS ITVELNLDGTGIVDVSTGVPFFDHMLTALGSHASFDL \\
\hline & & $\begin{array}{lll}61 & 12\end{array}$ \\
\hline & $(54)$ & DVKAKGDVEIDAHHTVEDTAIVFGQALGQALSDKKGIRRFGDAFI PMDETLAHASVDVS \\
\hline-10 & $(58)$ & TVRTTGDVEIEAHHTIEDTAIALGAALGQALGDKRGIRRFGDAFI PMDETLAHAAVDVSG \\
\hline $37 \mathrm{Rv}$ & $(61)$ & TVRATGDVEIEAHHTIEDTAIALGTALGQALGDKRGIRRFGDAFIPMDETLAHAAVDLSG \\
\hline NoC & $(59)$ & SVRAEGDIQIEAHHTVEDTAIVFGQALGKALGDKAGIRRFGDAFI PMDETLAHAAVDVSG \\
\hline \multirow[t]{2}{*}{ RHA1 } & $(54)$ & TVHAKGDIEIEAHHTVEDTSIVLGQALGQALGDKKGIRRFGDAFI PMDETLAHASVDVSG \\
\hline & & 121 \\
\hline & $(114)$ & RPYCVHTGEPDYMVHSVIGG-------YPGVPYHAVINRHVFESIALNARIALHVRVLYG \\
\hline-10 & $(118)$ & RPYCVHSGEPDHLQHSTIAG--------SSVPYHTVINRHVFESLAMNARIALHVRVLYG \\
\hline $37 \mathrm{Rv}$ & $(121)$ & RPYCVHTGEPDHLQHTTIAG--------SSVPYHTVINRHVFESLAANARIALHVRVLYG \\
\hline NoC & $(119)$ & RPYCVHTGEPEHLLHAVIPGSPVRGTGEPGAPYSTVLNRHVFES IALNARIALHVRVLYG \\
\hline \multirow[t]{2}{*}{ RHA1 } & $(114)$ & RPYCVHTGEPEHLLHSVIGG-------YPGVPYATVINRHVFESIALNARIALHVRVLYG \\
\hline & & 181 \\
\hline & $(167)$ & RDQHHITEAEYKAVARALREAVEPDPRVSGVPSTKGTL \\
\hline $\mathrm{K}-10$ & $(170)$ & RDPHHITEAQYKAVARALRQAVEPDPRVSDVPSTKGVL \\
\hline 7Rv & $(173)$ & RDPHHITEAQYKAVARALRQAVEPDPRVSGVPSTKGAL \\
\hline & $(179)$ & RDQHHVTEAEFKAVARALRAAVEFDPRVSGVPSTKGTL \\
\hline & $(167)$ & RDQHHITEAEFKAVARALREAVEPDPRVTGVPSTKGSL \\
\hline
\end{tabular}

Figure 4.21. Alignment of a putative HisB sequence from $R$. erythropolis SQ1 with HisB sequences from Rhodococcus sp. RHA1, Nocardia farcinica IFM 10152 (Noc), Mycobacterium avium subsp. paratuberculosis K-10 and M. tuberculosis H37Rv. Grey background, $>50 \%$ identity; green background, identical residues. 


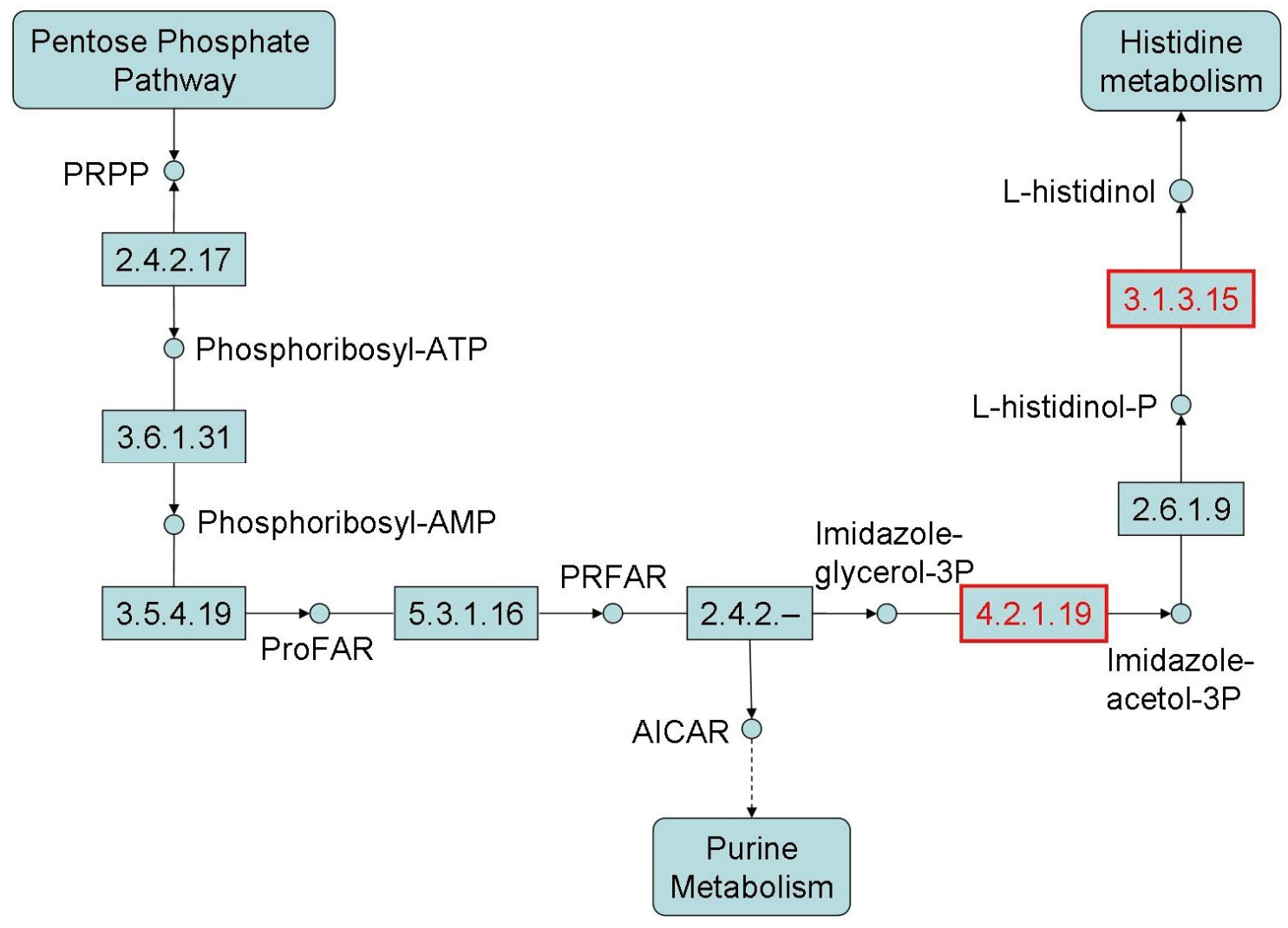

Figure 4.22. Steps catalysed by HisB (highlighted in red) in the histidine anabolic pathway [adapted from KEGG (Kanehisa et al., 2006)]. 
HisB is encoded by the gene hisB, and is part of the his operon. Histidine metabolism has been a focus point of genetics for over forty years, contributing to the early hypothesis that prokaryotes possess genes arranged in operons. The histidine operon has since been shown to be present in most bacteria studied, including the actinomycete Streptomyces coelicolor (Alifano et al., 1996), Mycobacterium smegmatis and $M$. tuberculosis (Parish et al., 1997). The organisation of the his operon is remarkably similar across genus and species, as illustrated in Figure 4.23, and has been commented on extensively in the literature (Price et al., 2006). From a genomic perspective, hisB is found upstream of hisH and hisA. It is interesting to note that Rhodococcus sp. RHA1, M. smegmatis and M. tuberculosis carry an inositol-monophosphatase between hisA and hisF which is involved in cell-wall synthesis (Parish et al., 1997). Although the genes his $B$, hisH and hisA are found in the same order in all organisms studied so far (Alifano et al., 1996), interestingly, the hisBHA sequence is interrupted in Nocardia farcinica IFM 10152, Streptomyces avermitilis MA-4680 and Rhodococcus sp. RHA1 (Ishikawa et al., 2004; McLeod et al., 2006; Omura et al., 2001). In Nocardia, the ORF nfa18490 lies between hisH and hisA. In Streptomyces, the ORF SAV6156 lies between hisB and hisH. These two ORFs bear no similarity and are of unknown function. Rhodococcus sp. RHA1 seems to be quite unique in having two ORFs inserted between hisB and his $H$ (ro01020 and ro01027) as well as one between hisH and hisA (ro01025). In R. erythropolis SQ1, the remaining 598 nucleotides of sequence information obtained for mutant 3B4 located downstream of the putative hisB gene are highly similar (over $70 \%$ identity) to the corresponding sequence in Rhodococcus RHA1. This would suggest that Rhodococcus RHA1 and R. erythropolis SQ1 share a similar organisation for the his operon. Predicted proteins encoded by ORF nfa18490, SAV6156, RHA1_ro01025 and RHA1_ro01028 are of unknown function, while RHA1_ro01027 is a putative major facilitator family transporter (McLeod et al., 2006). 
As was observed for the his $A$ mutants, the hisB mutant is auxotrophic for histidine in that normal growth was achieved when CDM II was supplemented with histidine (data not shown). 


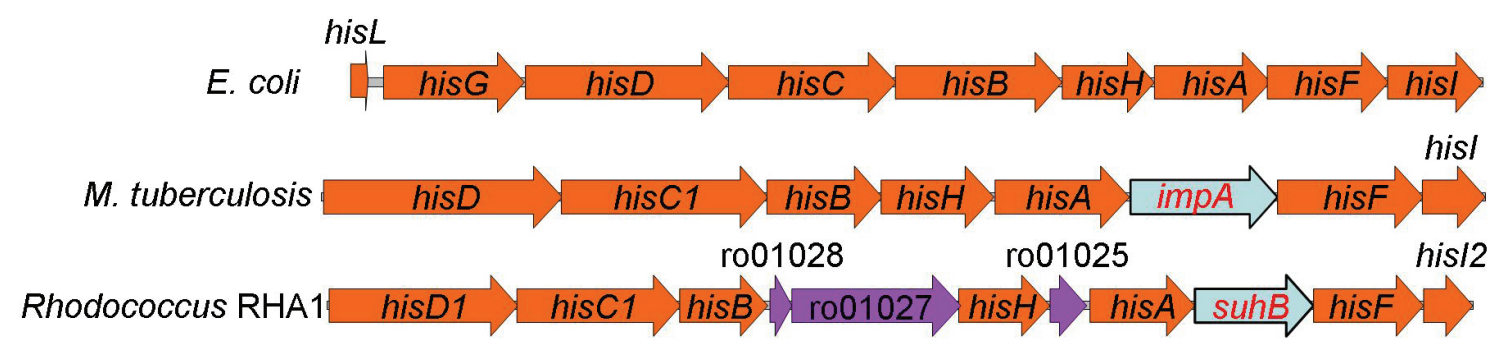

Figure 4.23. Histidine synthesis operons of $E$. coli K-12, M. tuberculosis H37Rv and Rhodococcus sp. RHA1. The purple arrows show open reading frames encoding proteins of unknown function. impA (M. tuberculosis) and suhB (Rhodococcus) both encode an inositol-monophosphatase specific to actinobacteria. 


\subsubsection{Serine/glycine auxotrophic serA mutant: $6 E 6$}

Mutant 6E6 failed to grow after 14 days incubation in either CDM $1 \%$ glucose or CDM $0.1 \%$ glucose. In contrast, wild-type $R$. erythropolis SQ1 gave strong growth at each sampling point. However, the wild-type phenotype was observed when mutant 6E6 was grown in LB broth. DNA from mutant 6E6 was isolated and processed in the same way as the mutants previously described and an $831 \mathrm{bp}$ sequence was retrieved (Figure 4.24). Although sequence data was obtained for regions both upstream and downstream of the transposon insertion point, only one restriction site could be located in the sequence obtained. A similar situation arose with mutants 1B3 and 4G11 restricted with EcoRI (section 4.4.1). Interestingly, a $10 \mathrm{bp}$ repeat sequence, TCCTCGCTCT, was observed at the transposon insertion point.

The nucleotide sequence obtained was translated and analysed using BLASTX against the non-redundant protein database at NCBI. Strong similarity was found between the sequence encoded by ORF 1 and the sequence of the D-3-phosphoglycerate dehydrogenase (PGDH, encoded by ser $A$ genes) of several mycobacteria (scores $>300$ and $E$-values $<2 \times 10^{-80}$ ). Each ORF was translated using the bacterial genetic code and submitted to a BLASTP search. ORF 1 was found to be the only one with significant similarity to proteins in the database, specifically to the D-3-phosphoglycerate dehydrogenase (EC 1.1.1.95, SerA).

The $831 \mathrm{bp}$ sequence obtained codes for the C-terminal 271 amino acids only, corresponding to approximately half the size of SerA proteins reported to date (505$531 \mathrm{aa})$. The sequence from 1 to $831 \mathrm{bp}$ was therefore translated in frame +1 to yield a 276 amino acid sequence that was aligned using Align to the best BLAST hits (Figure 4.25). An identity of $63 \%$ was calculated (using Align) between the amino acid 
sequence obtained from $R$. erythropolis SQ1 and the C-terminal 276 amino acids of SerA from M. tuberculosis H37Rv. 


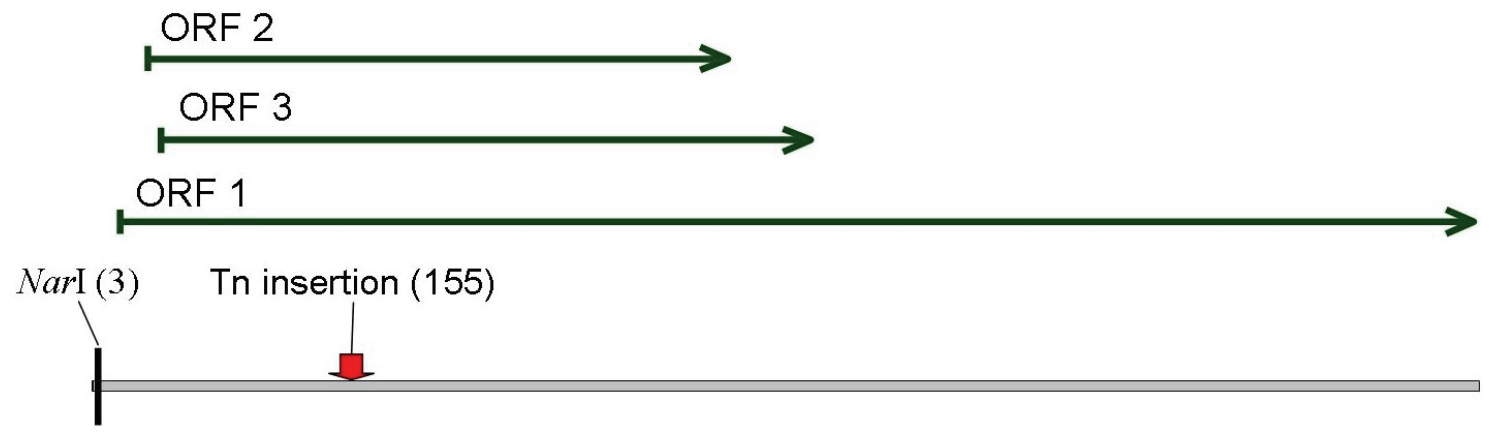

\begin{tabular}{|lclc|}
\hline Open reading frame & Position & Strand & Size \\
\hline ORF 1 & $16-828$ & Sense & $813 \mathrm{bp}$ \\
ORF 2 & $33-380$ & Sense & $348 \mathrm{bp}$ \\
ORF 3 & $41-430$ & Sense & $390 \mathrm{bp}$ \\
\hline
\end{tabular}

Figure 4.24. Schematic representation of the 831 bp sequence retrieved for mutant 6E6.

The NarI restriction site used to retrieve the sequence is shown, as well as putative open reading frames predicted by ORF finder and the transposon insertion site. Size, position and strand of the ORFs identified in sequence are shown in the table.

ORFs were identified with the ORF Finder function of Vector NTI (Invitrogen, http://www.invitrogen.com). 
SQ1

H37R

PYR-1

NoC

RHA1

$\mathrm{SQ1}$

H37Rv (59)

PYR-1

NoC

RHA 1

(1)

--MVSLPVVLIADKLAPSTVAALGDQVEVRWVDGPDRDKLLAAVPEADALLVRSATTVDA

(1) ---MSLPVVLIADKLAQSTVEALGDQVEVRWVDGPDREKLLAAVADADALLVRSATTVDA

(1) MSQAGRPVVLIADKLAQSTVDALGDGVEVRWVDGPDRPALLAAVPEADALLVRSATTVDA

(1) MSQPGRPVVLIADKLAPSTVEALGDGVEVRWVDGPDRPALLAAVPEADAILVRSATTVDA 61

120

(1) - - -

(59) EVLAAAPKLKIVARAGVGLDNVDVDAATARGVLVVNAPTSNI HSAAEHALALLLAASRQI

(58) EVLAAAPKLKIVARAGVGLDNVDVDAATARGVLVVNAPTSNI HSAAEHALALLLAAARQ I

(61) EVLEAGKNLKIVARAGVGLDNVDVPAATERGVMVVNAPTSN I HTAAEHAVTLLLAAARQ I

(61) EVLAAGTKLKIVARAGVGLDNVDVAAATERGVMVVNAPTSNI HTAAEHAVALMLATARQI 121

$\mathrm{S} Q 1$

(1)

H37Rv (119)

PYR-1 (118)

NOC (121)

PAADASLREHTWKRSSFSGTEIFGKTVGVVGLGRIGQLVAQRIAAFGAYVVAYDPYVSPA PAADATLREHSWKRSSFSGTEIFGKTVGVVGLGRIGQLVAQRLAAFGAH I TAYDPYVSHA PAADATLREHTẄQRSKFNGVEIYGKTVGVVGLGRIGQLFAARLAAFETKI IAYDPYVSPA PAADATLRDREWKRSKFNGVEIFGKTVGVVGLGRIGQLFAQRLAAFETHVIAYDPYVSAA 181

240

SQ1

(1)

H37RV (179)

PYR-1 (178)

NOC (181)

RHA1 (181)

RAAQLGIELLSLDDLLARADF ISVHLPKTPETAGLIDKEALAKTKPGVIIVNAARGGLVD RAAQLGIELLTLDELLGRADF ISVHLPKTKETAGLIGKEALAKTKP GVI IVNAARGGLID RAAQLGIELVTLDELLGRADLISVHLPKTPETKGLLSKEKLALTKKGVI IVNAARGGLID RAAQLGIELVTLDELLERADLISVHLPKTPETKGLLGTENLAKTKKGVVIVNAARGGLID 241

SQ1 (1) -----------------VFSTEPCTDSKLFELDNVVVTPHLGASTSEAQDRAGIDVAK H37RV (239) EAALADAITGGHVRAAGLDVFATEPCTDSPLFELAQVVVTPHLGASTAEAQDRAGTDVAE PYR-1 (238) EAALADA INSGHVRGAGLDVFSTEPCTDSPLFELPQVVVTPHLGASTVEAQDRAGTDVAA NOC (241) EQALADAITSGHVRAAGIDVFETEPCTDSPLFELPQVVVTPHLGASTTEAQDRAGTDVAK RHA1 (241) EAALAEAIKSGHVRAAGLDVFETEPCTDSPLFDLPEVVVTPHLGASTTEAQDRAGTDVAK 301

360

SQ1 (42) SVLLALAGEFVPEAVNVSGGPVGEEVAPWLELVRKLGLLAATLSPEAVQTVQVVATGELS

H37RV (299) SVRLALAGEFVPDAVNVGGGVVNEEVAPWLDLVRKLGVLAGVLSDELPVSLSVQVRGELA

PYR-1 (298) SVKLALAGEFVPDAVNVGGGAVGEEVAPWLDLVRKLGLLVGVLSSEP PVSLQVQVQGELA

NOC (301) SVLLALAGEFVPGAVNVTGGAVSDEVAPWLEIVRKQGALVGALSDELPVSVEVQVRGELA

RHA1 (301) SVLLALAGDFVPDAVNVSGGAVGEEVAPWLEIVRKQGVLIGALSGELPVNLSVDVRGELA 361

420

SQ1 (102) AETVDILGLAALRGVFSASSDEAVTFVNAPALAEQRGVTVSVEKHSEALAHRSAVEVRAV

H37RV (359) AEEVEVLRLSALRGLFSAVIEDAVTFVNAPALAAERGVTAEICKASESPNHRSVVDVRAV

PYR-1 (358) SEEVEVLKLSALRGLFSAVIEHPVTFVNAPALASERGVEAS ITTATESANHRSVVDVRAV

NOC (361) AEDVAVLELSALRGIFSALVEDQVTFVNAPALAKERGISVEVTTASESPSHRSVVDLRAV

RHA1 (361) SEDVEVLALSALRGVFSAVIEDAVTFVNAPALAEERGVTAEVTKAAESPNHRSVVDLRAV 421

480

SQ1 (162) AADGTVTSVTGALTGLQQVEKIVNINGRS FDLRAEGHNIVVH-YSDRPGVLGVLGTVLGN

H37RV (419) GADGSVVTVSGTLYGPQLSQKIVQINGRHFDLRAQGINLI IH-YVDRPGALGKIGTLLGT

PYR-1 (418) AADGSTVNVAGTLTGPQLVEKIVQINGRNLELRAEGVNLI IN-YDDQPGALGKIGTLLGG

NOC (421) FGDGRTLNVAGTLTEPQQVQKIVNINGRNYDMRAEGLNLAVLNYDDRPGALGKIGTRLGE

RHA1 (421) FGDGSVINVSGTLTGPQQVEKIVNINGRNEELRAEGLNLVVN-YTDQPGALGKIGTQLGN 481 532

SQ1 (221) AGVDILAAALSQDAEGEGATVILRVDRVVGDAEVEAIVSQLDARVAQVDLS H37RV (478) AGVNIQAAQLSEDAEGPGATILLRLDQDVPDDVRTAIAAAVDAYKLEVVDLS

PYR-1 (477) AAVNILAAQLSQDADGIGATVMLRLDREVPGEVLAAIGRDVNAVTLEVVDLT

NOC (481) ADIDILAAQLSQDIDKEGATVILRVNKPVPADVQTAIAEAVGAAKIALVDLF

RHA1 480) AGIDIQAAQLSQDAEGEGATILLRVDREVPSEVRDAISTAVGATKIELVNLA

Figure 4.25. Alignment of a putative SerA sequence from $R$. erythropolis SQ1 with sequences from Rhodococcus sp. RHA1, M. tuberculosis strain H37Rv, $M$. vanbaalenii PYR-1 and $N$. farcinica IFM 10152.

Grey background, $>50 \%$ identity; green background, identical residues. 
SerA catalyses the conversion of D-3-phosphoglycerate to 3-phospho-hydroxypyruvic acid (or hydroxypyruvic acid phosphate) and vice versa. It is one of the first steps in the biosynthesis of serine from glycerate (Figure 4.26) (Ichihara \& Greenberg, 1957; Walsh \& Sallach, 1966). Since SerA appears essential to the synthesis of serine in M. tuberculosis (Sassetti et al., 2003) and could be involved in the biosynthesis of glycine and threonine, a short supplementation study was performed. Growth of mutant 6E6 was assessed in CDM II 1\% glucose broth supplemented with glycine, serine, threonine, and combinations of these amino acids (all at concentrations of $100 \mu \mathrm{g} \mathrm{ml}^{-1}$ ) (Table 4.6). It is interesting to see that both serine and glycine restored the ability of the mutant to grow in chemically defined medium, presumably because of the possibility to synthesise serine from glycine (EC 2.1.2.1, Figure 4.25). The seemingly complete inhibition of growth by threonine was not completely unexpected, considering its toxic effects on other organisms (Eccleston \& Kelly, 1973; Lamb \& Bott, 1979). 


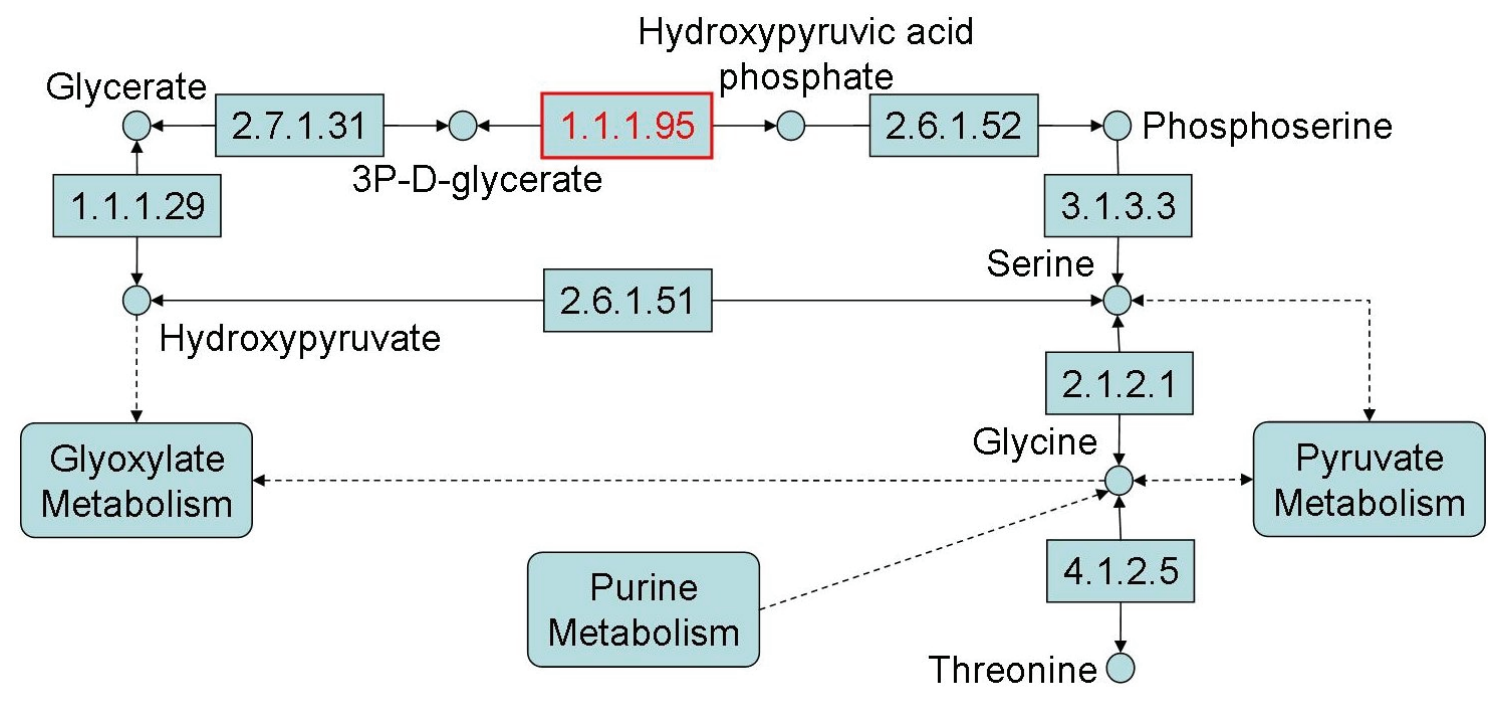

Figure 4.26. Position of phosphoglycerate dehydrogenase in a simplified glycine, serine and threonine metabolism pathway [adapted from the Rhodococcus sp. RHA1 pathway from KEGG (Kanehisa et al., 2006)]. 


\begin{tabular}{|lc|}
\hline Growth medium (CDM II 1\% glucose) & Growth density \\
\hline Non-supplemented & - \\
+ threonine & - \\
+ serine & ++ \\
+ glycine & +++ \\
+ glycine + serine & +++ \\
+ glycine + threonine & - \\
+ serine + threonine & - \\
+ glycine + serine + threonine & - \\
\hline
\end{tabular}

Table 4.6. Growth of mutant 6E6 in CDM $1 \%$ glucose broth supplemented with serine, glycine and threonine $\left(100 \mu \mathrm{g} \mathrm{ml}^{-1}\right)$.

- , no visible growth; + to +++ , increasing growth. 
A gene database search (NCBI) revealed that there are at least two genes with the same putative function in Rhodococcus sp. RHA1, serA2 and serA3, and a further three putative serA genes (RHA1_ro04630, RHA1_ro01308 and RHA1_ro02790). Since mutant 6E6 is auxotrophic for serine/glycine, it seems that only the gene disrupted by transposon insertion is expressed in CDM IIA, in other words the additional gene copies are not fulfilling the activity of the inactivated gene under these growth conditions. The possession of multiple ser $A$ or ser $A$-like genes is common. For instance, two ser $A$ genes are found in M. tuberculosis H37Rv and M. bovis AF2122/97 (serA1 and serA2); one gene with proven function, in addition to a putative gene are found in Nocardia farcinica IFM 10152 (serA and nfa13640) and M. tuberculosis CDC1551 (serA, identical to serA1 from M. tuberculosis and M. bovis and MT0753). 


\section{Chapter V \\ Discussion}


Although the starvation or non-growth state is probably the most common physiological state of bacteria (Morita, 1993), it has been studied in relatively few organisms. In spite of its importance in pathogenesis, bioremediation and several industrial processes, limited research has been performed on members of the genus Rhodococcus under starvation conditions. The objectives of this study were to analyse the physiological adaptation of Rhodococcus to starvation/stationary phase, and to generate a bank of mutants to identify genetic elements involved in this adaptation. The study organism, R. erythropolis SQ1, was chosen based on ease of growth in chemically defined medium, antibiotic sensitivity profile (of relevance as selective pressure in DNA transformation experiments) and transformation efficiency by electroporation.

In this study, it was shown that, similar to Vibrio, S. aureus and M. tuberculosis (Jenkins et al., 1988; Nyka, 1974; Nyström, 1999; Watson et al., 1998b), $R$. erythropolis SQ1 can survive carbon starvation or nutrient-induced stationary phase for a prolonged period of time. It was found that $R$. erythropolis SQ1 can survive for at least 43 days in LB and distilled water, and at least 65 days in chemically defined medium $(\mathrm{CDM})$ containing high $(1 \% \mathrm{w} / \mathrm{v}, 55 \mathrm{mM})$ or low $(0.1 \% \mathrm{w} / \mathrm{v}, 5.5 \mathrm{mM})$ concentrations of glucose. Interestingly, growth in a low-energy medium $(5.5 \mathrm{mM}$ glucose medium) enabled $100 \%$ of the cells in culture (approx. $1.2 \times 10^{9} \mathrm{CFU} / \mathrm{ml}$ ) to form colonies on agar, even after 65 days static incubation (data not shown). Viable cell counts, measured by plating on LB agar, remained more or less constant (variation $\leq 1 \log$ ), regardless of the medium in which the cells were grown. Other organisms were shown to survive long periods of time under similar conditions, e.g. Listeria monocytogenes, Staphylococcus aureus, Mycobacterium tuberculosis and Rhodococcus rhodochrous (Herbert \& Foster, 2001; Shleeva et al., 2002; Watson et al., 1998b). It was verified that, during long-term stationary phase, the glucose was exhausted from 
chemically defined medium $(<0.1 \mathrm{mM}$, as measured by a colorimetric method) between 6 and 7 incubation in $0.1 \% \mathrm{CDM}$, and between 7 and 13 days incubation in $1 \% \mathrm{CDM}$, respectively. This shows that $R$. erythropolis SQ1 could survive for long stretches of time in a medium in which there was no source of energy. Additional samples would be necessary to pinpoint the exact time at which glucose was depleted from the medium. The growth rate in exponential phase was found to be much lower when $R$. erythropolis SQ1 was grown in $0.1 \% \mathrm{CDM}$ than when grown in $1 \% \mathrm{CDM}$ (approx. $0.094 \mathrm{hr}^{-1} \mathrm{vs}$ $0.17 \mathrm{hr}^{-1}$, respectively). More frequent sampling would have however been necessary for a more accurate figure to be determined. The observations reported in this study are true of $R$. erythropolis grown with glucose as the sole carbon source. It would be interesting in the future to compare behaviours using alternative carbon and/or energy sources. The modified chemically defined medium described in this work could also be applied to studies investigating other growth-limiting nutrients or elements, such as nitrogen and phosphorous, or trace metals and vitamins. Furthermore, it would be of interest to study the physiological status of immobilised cells, as these have been proposed for use in several industrial processes (see e.g. Begoña Prieto et al., 2002; Kitova et al., 2002; Kitova et al., 2004; Naito et al., 2001; Pirog et al., 2005; Prieto et al., 2002).

Interestingly, the culturability of $R$. erythropolis SQ1 dropped dramatically (by $4 \operatorname{logs}$ ) when flasks were transferred from shaking to static incubation, but did not disappear altogether, as cells could still yield colonies on LB agar (approx. $4 \times 10^{5} \mathrm{CFU} / \mathrm{ml}$ ). This behaviour was observed only in high-glucose $(1 \%)$ medium. Transfer to static incubation is used to produce dormant cells in M. tuberculosis and M. bovis growing in Dubos Tween-albumin broth (0.75\% glucose, w/v) (Lim et al., 1999; Wayne \& Hayes, 1996). The same effect was observed in Rhodococcus rhodochrous grown in modified 
Sauton's medium (approx. $6 \%$ glycerol, v/v) (Shleeva et al., 2004; Shleeva et al., 2002). Significantly, $R$. erythropolis SQ1 grown in CDM $0.1 \%$ glucose did not exhibit any drop in culturability upon transfer to static incubation. It can be hypothesised that the lower concentrations of carbon source/energy primed the bacteria for viability in low-oxygen conditions. Previous and current studies on $R$. erythropolis have focussed on high-oxygen conditions for industrial processes (Gomez et al., 2006; Kretschmer \& Wagner, 1980a; Kretschmer \& Wagner, 1980b; Santos et al., 2006). Further work on the survival of $R$. erythropolis in microaerophilic and/or anaerobic conditions would shed light on its survival in for example the deep sea (Heald et al., 2001).

An association between carbon starvation, or entry into stationary phase, and increased resistance to a range of environmental stresses has been reported for several bacterial genera including Vibrio, Staphylococcus aureus and mycobacteria (Clements \& Foster, 1998; Kjelleberg et al., 1993; Nyka, 1974). The second objective of this study was to determine whether glucose-starved $R$. erythropolis SQ1 possesses enhanced resistance to heat and oxidative stress. The most dramatic increase in resistance was to heat shock: after 5 min exposure to $57^{\circ} \mathrm{C}$, no exponential phase cells survived, whereas nearly $40 \%$ of early stationary phase cells did. The resistance afforded by carbon starvation to oxidative stress was less dramatic, with almost $50 \%$ of exponential phase cells surviving $5 \mathrm{~min}$ in the presence of $400 \mathrm{mM}$ tBHP, compared with $63 \%$ of early stationary phase cells. After 10 min of oxidative stress, $40 \%$ of early stationary phase cells survived, vs $7 \%$ of exponential phase cells. These results are broadly similar to those obtained in other organisms, e.g. Staphylococcus aureus and Escherichia coli (Jenkins et al., 1988; Watson et al., 1998b), although like-for-like comparisons are difficult due to the differences in protocol and the fact that different bacteria have different innate sensitivities to stress. Although the tBHP concentration used in this 
study was found to be adequate to investigate the cross-protection afforded by entry into stationary phase/starvation, further investigations could be carried out with different concentrations, in particular in the $200-500 \mathrm{mM}$ range. This would help in determining the mode of the killing effect of tBHP on $R$. erythropolis SQ1, as it has been demonstrated previously that hydrogen peroxide has a bimodal killing effect on cells of E. coli (Imlay \& Linn, 1986).

To investigate whether protein synthesis was involved in the stress resistance mechanisms, chloramphenicol, a bacteriostatic antibiotic that inhibits protein synthesis, was added to the test sample before exposure to the stress. Interestingly, for R. erythropolis SQ1, chloramphenicol treatment did not significantly reduce the degree of resistance of exponential or early stationary cultures to heat shock. This is in contrast to previous studies performed on E. coli in which it was found to restore exponentialphase sensitivity to stationary phase E. coli (Jenkins et al., 1988). It is possible that, in the experiments performed in this study, the timing of exposure to chloramphenicol was not optimal for observation of induction of cross protection and its inhibition by chloramphenicol. The fact that no reduction in heat shock resistance was observed would lead one to conclude that the proteins that protect against heat shock have already been synthesised prior to chloramphenicol treatment. Since exponential phase cells were however sensitive to a heat shock of $57^{\circ} \mathrm{C}$, it is probable that the heat shock proteins were synthesised between the two sampling points, at 2 and 6 days of growth.

For oxidative stress, on the other hand, chloramphenicol treatment decreased exponential phase $R$. erythropolis resistance to tBHP. A transitory effect was observed in chloramphenicol-treated early stationary phase cells, their sensitivity to tBHP at 5 min exposure was the same as untreated exponential phase cells. However, cell 
resistance to tBHP at 10 and 15 min was similar to that of untreated early stationary phase cells. It is unclear how resistance to oxidative stress could be restored in the absence of protein synthesis. The point made above about the time of sampling is also valid here. It is well-known that different enzymes are produced by bacteria to protect against peroxides and superoxides, the most well known are catalase and superoxide dismutase, respectively. tBHP is a peroxide, it is quite possible that a superoxide such as veratrate would elicit a different response in $R$. erythropolis SQ1.

Taken together, these results show that $R$. erythropolis SQ1 reacts to carbon depletion in the medium by triggering a starvation survival response, resulting in long-term survival and cross-protection. As long as aeration is maintained in liquid culture, the cells can form colonies on agar plates. The inability to form colonies on agar plates was observed only in high-energy medium ( $55 \mathrm{mM}$ glucose medium), and only when the cells where transferred to static incubation in early stationary phase.

The behaviour of the cells treated with chloramphenicol can be explained in several ways. Firstly, it is possible that the experimental setup was not appropriate, although the concentration of chloramphenicol used was shown to inhibit $R$. erythropolis SQ1 growth both on agar and in broth, and was shown to act instantly by continuously monitoring the $\mathrm{OD}_{600 \mathrm{~nm}}$ of a test culture while chloramphenicol was added at various concentrations. Secondly, it is possible that heat protection proteins (e.g. chaperones) are constitutively expressed in high concentration in $R$. erythropolis SQ1 grown in lowenergy medium (5.5 mM glucose); growth in low-energy medium was shown to protect the cells against static incubation, and this may have other cross-protecting effects. This could be investigated by increasing the quantity of glucose in the medium. Under the 
conditions used in this study, chloramphenicol did inhibit the development of resistance to oxidative stress, particularly noticeable in the case of exponential phase cells.

The next step in the study was to generate a mutant bank that could be screened for stationary phase survival deficient mutants, in order to then identify the genes involved. Once electroporation settings and recovery conditions had been optimised, the generation of the mutant bank was rather straightforward. The Tn<KAN2 $>$ kit (Epicentre) was selected for generation of a mutant bank of $R$. erythropolis SQ1 since it had been used previously to generate mutants of $R$. rhodochrous, $R$. equi and R. erythropolis (Fernandes et al., 2001; Mangan \& Meijer, 2001; Tanaka et al., 2002) with no particular problems reported. The number of mutants obtained in this study was comparable to that obtained for $R$. equi and R. rhodochrous (approx. 600 and 1500, respectively, Fernandes et al., 2001; Mangan \& Meijer, 2001), but disappointing compared with that obtained for $R$. erythropolis KA2-5-1 (approx. 46,000, Tanaka et al., 2002).

Following a simple growth experiment in microtitre plates, it was possible to isolate mutants with growth defects. All mutants generated were screened for stationary phase/starvation survival using a method similar to that of Uhde et al. (1997). The results for each mutant were recorded on the basis of appearance of growth on LB agar after 1, 8, 14 and 22 days incubation in CDM 1 and $0.1 \%$ glucose broth. Mutants were selected for further study based on the extent and presence of growth in stationary phase. From 898 mutants screened, four mutants, 1B2, 1H1, 4G6 and 10D3, were of particular interest with regards to stationary phase/starvation survival. When grown in $500 \mathrm{ml}$ glass flasks in $100 \mathrm{ml} \mathrm{CDM} 1$ or $0.1 \%$ glucose and compared with the behaviour of the wild-type $R$. erythropolis SQ1 grown under the same conditions the 
following observations were made. The culturability of mutants 4G6 and 10D3 dropped to 0.01 and $0.1 \%$ of the maximum $\mathrm{CFU} / \mathrm{ml}$ at 27 days incubation, respectively, when grown in $1 \%$ glucose medium. A drop in culturability was also observed in $1 \%$ glucose medium for mutants $1 \mathrm{~B} 2$ and $1 \mathrm{H} 1$, to 3 and $2.5 \%$ of the maximum $\mathrm{CFU} / \mathrm{ml}$ at 27 days incubation, respectively. Interestingly, no significant drop in culturability could be observed for any of these four mutants when grown in CDM $0.1 \%$ glucose.

One aim of this study was to identify genetic elements involved in the stationary phase and starvation survival of $R$. erythropolis SQ1. An essential step was therefore to isolate DNA from the mutants. Many protocols reported in the literature for the isolation of genomic DNA from Rhodococcus are based on phenol extractions and proteinase $\mathrm{K}$ treatment (see e.g. Treadway et al., 1999; Veselý et al., 2003). Interestingly, protocols include lysozyme, even though it has been reported that several rhodococci are resistant to lysozyme (Mitani et al., 2005; Solovykh et al., 2004). In this study it was found that, although it was possible to lyse $R$. erythropolis SQ1 cells with similar methods, the amount of protein present in the samples of some mutants made it very difficult to isolate clean genomic DNA. Large volumes of reagents and enzyme were required, and it was not possible to design a standard protocol that could be used for all mutants. Therefore a kit (FastDNA SPIN kit for soil; Qbiogene) using a mechanical cell disruption method (glass beads) and a resin-based DNA purification method was used, as advised by Dr Herron, University of Strathclyde, Glasgow, yielding consistently higher quality genomic DNA for further molecular manipulation.

In order to recover the sequences flanking the transposon insertion site in each of these mutants, several approaches were tested. Among these were two methods, arbitrarily primed PCR (AP-PCR) and random amplification of transposon ends PCR (RATE- 
PCR), using either degenerate primers or non-stringent binding conditions, respectively (Caetano-Anollés, 1993; Ducey \& Dyer, 2002). In the first method, PCR is performed on the template with degenerate primers, a smear of amplified DNA is produced, as verified by agarose electrophoresis, and specific primers are then used to amplify the sequence of interest. This technique has been successfully applied to Pseudomonas fluorescens and Vibrio cholerae for example (Burrus \& Waldor, 2003; O'Toole \& Kolter, 1998), but the amplicons are usually short (200-400 bp). Short sequences could be generated with mutant $4 \mathrm{G} 11$, but were deemed too short to give a good overview of the genome segment that had been disrupted by transposon insertion.

RATE-PCR has been used to recover transposon-flanking DNA from Neisseria gonorrhoeae (Ducey \& Dyer, 2002). This method relies on lowering the annealing temperature during PCR, so that non-specific binding occurs. When applied to the analyses of mutants in this study, many bands of varying sizes were produced for each PCR, but not all were amplicons of the transposon-flanking DNA. By the random nature of this technique, the results could not be reproduced, in that bands obtained in repeated PCR reactions in fact consisted of different amplicons. These problems might also be related to the discrepancy in $\mathrm{G}+\mathrm{C}$ content between the primer and the rhodococcal DNA. The primer is specific to an end of the transposon and therefore has a $\mathrm{G}+\mathrm{C}$ content of approximately $50 \%$, vs approximately $70 \%$ for Rhodococcus DNA. It would therefore seem logical that low $-\mathrm{G}+\mathrm{C}$ areas of the rhodococcal DNA would be amplified preferentially, even if the transposon inserted elsewhere.

Since neither of the above methods was successful, a third method was tested based on the use of inverse PCR. This yielded much better results (sequences of up to $1 \mathrm{~kb}$ were obtained) and allowed DNA amplification products from 9 mutants (out of 16 selected) 
to be cloned and sequenced. The limitation of this method was finding for each mutant a restriction enzyme that yields transposon-containing fragments of an appropriate size for religation and subsequent PCR amplification. This meant that many restriction endonucleases had to be systematically tested on each mutant, as there was no easy or rapid way in which to predict which enzyme would yield the appropriate fragments. An added difficulty was the amplification of a low-copy number, high $\mathrm{G}+\mathrm{C}$ DNA sequence. Although modern Taq polymerase kits are very efficient and effective, some DNA regions could still not be amplified and/or sequenced, even using "high-fidelity" or "high $\mathrm{G}+\mathrm{C}$ " enzymes (e.g. for mutant 1B2, attempts made were made with YBGCMix, Yorbio; Deep VentR, NEB; GC-rich PCR system, Roche). For such recalcitrant mutants there are additional alternative methods that could still be tested such as ligation-mediated PCR (LMPCR, Prod'hom et al., 1998). For LMPCR, the target DNA is digested with a restriction enzyme that cuts both within and outwith a known sequence, e.g. a transposon. An asymmetric, double-stranded, nonphosphorylated linker is then ligated to the restricted DNA. During the first PCR step, only a primer specific to the known sequence is used, thereby amplifying only the sequence that flanks the known sequence. A standard PCR is then performed, with primers specific to the known sequence and to the linker. Amplicons of mycobacterial sequences of up to $2.8 \mathrm{~kb}$ in size have been produced with this method (Prod'hom et al., 1998). However, the issues mentioned above regarding restriction fragment size, PCR amplification and sequencing of complex sequences would still apply, as it has been demonstrated that the failure rate of PCR increases with $\mathrm{G}+\mathrm{C}$ content and length of the target sequence (Benita et al., 2003).

An alternative method would be to use a transposon like EZ::Tn5 $<$ R6Kyori/KAN-2> (Epicentre) (it should be noted that this became available during the later stages of this 
project). As can be seen from the name of the transposon, it has been modified to include a plasmid origin of replication, ori. It is therefore possible to extract the genomic DNA from mutants of interest, restrict it, perform a self-ligation and electroporate the resulting plasmids into E. coli. When applied to a study of Rhodococcus erythropolis AN12 (Yang, 2006; Yang et al., 2007a), two such plasmids, of 11 and $16 \mathrm{~kb}$, were recovered. The use of this transposome bypasses the PCR step that was so problematic in the iPCR approach described above.

Starvation/stationary phase mutants were selected on the basis of non-recovery of growth on LB agar after incubation in chemically defined medium containing 1 or $0.1 \%$ glucose $(\mathrm{w} / \mathrm{v})$. Sixteen mutants, exhibiting weak or no growth on LB agar in at least one stage of growth in chemically defined medium, were selected for further study. Sequence data was obtained for nine mutants. Sequence analysis of several of the mutants revealed transposon insertion in biosynthetic genes that rendered them auxotrophic for histidine (insertions in his $A$ or hisB in the case of mutants 1B3, 3B10, 4G11, 10E4 and 3B4) and serine/glycine (insertion in serA, mutant 6E6). Addition of histidine in the growth medium restored wild-type growth in the mutants, thereby confirming the function of the genes disrupted. It is not clear whether mutants 1B3, 3B10, 4G11 and 10E4 are the result of separate transposon insertion events or clones of each other. Since the insertion was in the same locus for all four mutants, the clone explanation is probably the most likely. These auxotrophic mutants probably featured in the screening experiment because of carry-over of organic material from the master plate during replica plating. Since the volumes of medium used were small $(200 \mu 1)$, even very small amounts of carry-over would enable growth. The histidine mutants had inserts in genes that, in other organisms, are part of an operon. Interestingly, it seems that Rhodococcus RHA1 contains genes of unknown or unrelated functions in the his 
operon. More sequence data would be necessary to determine whether these genes are also in an operon in $R$. erythropolis SQ1. This is however very likely, as this operon is ancient and conserved in many species, including actinobacteria (Price et al., 2006). Various studies on the regulation of the his operon have revealed that, as expected, the histidine synthesis genes were repressed under stationary phase/starvation conditions in E. coli (hisF; Franchini \& Egli, 2006) and M. tuberculosis (hisA and hisI2; Betts et al., 2002). Surprisingly, the entire his operon was up-regulated in B. subtilis (hisZGDBHAFI; Eymann et al., 2002).

The serine/glycine auxotroph mutant (6E6) resulted from transposon insertion in a ser $A$ gene homologue. The protein encoded by $\operatorname{ser} A$ is a D-3-phosphoglycerate dehydrogenase that catalyses the first step of synthesis of serine from pyruvate. Since this pathway then leads to the synthesis of glycine and threonine, growth of the mutant was therefore tested in chemically defined medium containing serine, glycine, threonine or combinations of these amino acids. The growth of the serine/glycine auxotrophic mutant could be restored by addition of either serine or glycine, singly or together, but was completely inhibited by threonine. It is possible that the aspartokinase of R. erythropolis SQ1 is inhibited by threonine, as was described for Methylococcus capsulatus (Eccleston \& Kelly, 1973), thereby disrupting the synthesis of aspartic acid and aspartate-based amino acids (lysine, methionine, threonine and isoleucine). Eccleston and Kelly (1973) have described how methionine can relieve the inhibition caused by threonine but this was not attempted in this study. In a microarray experiment monitoring gene expression of E. coli grown in glucose-limited continuous culture, it was found that the gene $\operatorname{ser} A$ was down-regulated at both 40 and 500 hours incubation (Franchini \& Egli, 2006). 
One of the mutants that could not survive stationary phase was 4G6, with a transposon insertion in the gene $u v r B$. UvrB, the excinuclease $\mathrm{ABC}$ subunit $\mathrm{B}$, is an essential part of the DNA excision repair mechanism; therefore it is not surprising that this mutant would have difficulty in surviving stationary phase. It is known that, as cells stop growing, they accumulate mutations in the DNA (Bridges, 1998; Kivisaar, 2003). The role of $u v r B$ in stationary phase mutations is unclear, as it has been shown that $u v r B$ mutants accumulate mutations during stationary phase in E. coli (Vidal et al., 1998), but the opposite was observed in Pseudomonas putida (Tark et al., 2008). Furthermore, a Mycobacterium tuberculosis $u v r B$ mutant was found to be extremely sensitive to nitric oxide, and UvrB was essential for resistance to reactive oxygen and reactive nitrogen intermediates. The stationary phase survival of this mutant however was not investigated (Darwin \& Nathan, 2005). Interestingly, loss of viability was observed in the $u v r B \quad R$. erythropolis SQ1 mutant grown in CDM $1 \%$ glucose, but not in CDM $0.1 \%$ glucose. Two explanations can be advanced for this discrepancy. Firstly, the high cell concentration obtained in CDM $1 \%$ glucose might mean that high levels of oxidants and waste compounds have accumulated in the growth medium. As cells die and lyse, even more of these compounds would be released, hence increasing their concentration still further. Secondly, it is possible that growth in $0.1 \%$ glucose induced expression of alternative DNA repair mechanisms, or of DNA-protecting proteins such as Dps, as has been shown in Mycobacterium smegmatis (Gupta et al., 2002).

Mutant 10D3 was found to have the same growth profile as the UvrB mutant, although the transposon insertion does not seem to have disrupted a gene. The transposon is inserted immediately downstream of a putative guaB gene. Similar guaB genes are present in other organisms, but are not part of an operon, and are usually followed by another IMP dehydrogenase (guaB-like) and/or a cholesterol oxidase. The functions of 
these genes are unclear however, so the function disturbed in $R$. erythropolis SQ1 mutant 10D3 is unknown. It is possible that the decrease in viability of this mutant was due not to the disruption of a gene or regulatory sequence, but to overexpression of the kanamycin resistance gene of the transposon. The transposon inserted downstream of the guaB gene, a gene essential to the growth of the organism (Gil et al., 2004). It is therefore possible that the KAN2 gene came under regulation of the guaB promoter, resulting in overproduction of the kanamycin resistance protein (aminoglycoside $3^{\prime}$ phosphotransferase). Over-expression of foreign proteins has been shown to inhibit bacterial growth and induce an incomplete starvation survival response (Kurland \& Dong, 1996), that could affect the long-term stationary phase survival of cells grown in CDM $1 \%$ glucose. However, this seems unlikely, since the genes guaB and guaA were repressed in E. coli grown for $40 \mathrm{~h}$ in continuous culture in medium containing $0.01 \%$ glucose (Franchini \& Egli, 2006), and guaB was repressed in Bacillus subtilis in which stationary phase was artificially induced by the addition of norvaline (Eymann et al., 2002). Alternatively, the insertion could have affected the expression of the sequence downstream of the transposon insertion. Interestingly, the loss of culturability was observed only in CDM $1 \%$, but not in $0.1 \%$ glucose.

Mutant $1 \mathrm{H} 1$ was found to lose viability when grown in CDM $1 \%$. Interestingly, although the flanking sequence from mutant $1 \mathrm{H} 1$ could be amplified by iPCR using standard Taq polymerase and cloned, and subsequently amplified from the clone, it was not possible to obtain sequence data for one flanking sequence, despite several attempts. The transposon is inserted $272 \mathrm{nt}$ downstream of a gene encoding a putative phosphoglycerate mutase. The mutase identified is probably a fructose-2,6bisphosphatase involved in five-carbon sugar metabolism. Unfortunately sequence information was not obtained for the sequence downstream of the transposon insertion 
site; although in other organisms thioredoxin and cytochrome $c$ biogenesis genes have been identified. Closer analysis of the 200 nucleotide sequence immediately upstream of the transposon did reveal significant homology to putative thioredoxins and cytochrome $c$ biogenesis proteins of Rhodococcus RHA1 and Nocardia. It should be noted that in other actinobacteria such as Saccharopolyspora erythraea, Mycobacterium ulcerans and M. tuberculosis, it was found that the phosphoglycerate mutase gene is situated upstream of a putative thioredoxin protein/cytochrome $c$ biogenesis protein gene, in the same orientation. It is therefore possible that polar effects of transposon insertion could disturb the expression of these two genes. Interestingly, the phosphoglycerate mutase genes $\mathrm{pgm}$ and $y i b O$ were found to be increasingly expressed in norvaline-induced stationary phase in B. subtilis (Eymann et al., 2002) and in $500 \mathrm{~h}$ $0.01 \%$ glucose continuous culture E. coli (Franchini \& Egli, 2006). Indeed, many thioredoxin-related genes have been found to be induced by stationary phase; they are shown in Table 5.1. Thioredoxins are involved in many processes in bacteria, in particular DNA synthesis, protein repair, sulphur assimilation, cell division, energy transduction, transcriptional regulation and oxidative stress response (for a review, see Zeller \& Klug, 2006). During oxidative stress response, thioredoxins reduce disulfide bonds and scavenge reactive oxygen species. Interestingly, DsbE (a disulfide oxidoreductase) is involved in the maturation of cytochrome $c$ in E. coli (Fabianek et al., 1998). Cytochrome $c$ itself also seems to be involved in maintaining peroxidase and catalase activity in the periplasm (Goodhew et al., 1990).

There are relatively few rigorous studies in which carbon limitation is definitely the stationary phase-triggering factor (see e.g. van Overbeek et al., 1997). In many cases this is because the organism studied requires amino acid supplementation for growth (for example Seymour et al., 1996). The ability of $R$. erythropolis SQ1 to grow in a 
rather simple chemically defined medium where all important elements are supplied by inorganic salts is therefore important. The list of vitamins used as supplements in this study could probably be reduced drastically by further modification of the medium. However, it should be noted that, in the medium used in this study the concentration of vitamins used is so low that it is unlikely to support measurable growth. It is nonetheless challenging to screen for mutants deficient in carbon-starvation survival. This is due in no small part to the fact that many of these mutations are probably lethal, given the importance of carbon in every biological process. Different protocols could be used, that simulate "real-world" conditions more closely. For instance, the mutants could have been replica-plated to chemically defined medium agar plates containing decreasing quantities of glucose, or a selection of carbon sources. Investigation of longer term survival would also be interesting, although the growth advantage in stationary phase (GASP) phenotype would certainly eventually arise (Zinser \& Kolter, 1999; Zinser \& Kolter, 2004).

In all the mutants for which sequence information could be obtained, the transposon insertion was accompanied by the duplication of a short stretch of the target sequence (Figure 5.1). For all the mutants (with one exception), a textbook 9 bp repeated sequence, typical of Tn5 insertion sites (Goryshin et al., 1998), was observed. Only mutant 6 E6 contained a $10 \mathrm{bp}$ repeat, which could be explained by slippage of the transposase-DNA complex during insertion. Interestingly, $10 \mathrm{bp}$ is the kind of duplication seen at the insertion site of IS1166, as observed in R. erythropolis AJ270 and AJ300 (O'Mahony et al., 2005). Also, in mutant 4G6 (UvrB mutant) an imperfect duplication was observed, in that only 7 out of $9 \mathrm{bp}$ were identical. It is however plausible that this is a PCR artefact, rather than something directly resulting from the insertion mechanism. Interestingly, symmetry of the duplicated sequence was observed 
in a comprehensive study of Tn5 insertion in Streptomyces coelicolor, e.g. GCCCNGGGC (Herron et al., 2004), but no such symmetry was found in R. rhodochrous or $R$. equi Tn5 insertion sites (Fernandes et al., 2001; Mangan \& Meijer, 2001). This symmetry therefore seems to be limited to Streptomyces coelicolor. 


\begin{tabular}{|llll|}
\hline Gene & \multicolumn{1}{c}{ Function } & \multicolumn{1}{c|}{ Organism } & \multicolumn{1}{c|}{ Reference } \\
\hline$y f c F$ & GST enzyme with thioredoxin-like domain & E. coli & Weber et al. (2005) \\
$y n c G$ & GST with C-terminal thioredoxin-like and GST & E. coli & Weber et al. (2005) \\
& domains & & \\
$y g j K$ & GST enzyme with thioredoxin-like domain & E. coli & Weber et al. (2005) \\
$d s b G$ & Thiosulfide reductase & E. coli & Weber et al. (2005) \\
$d s b E$ & Disulfide oxidoreductase & E. coli & Franchini \& Egli (2006) \\
$y u m C$ & Thioredoxin reductase & B. subtilis & Eymann et al. (2002) \\
$\operatorname{trxA}$ & Thioredoxin & B. subtilis & Hecker \& Völker (1998) \\
$\operatorname{trxC}$ & Thioredoxin & M. tuberculosis & Betts et al. (2002) \\
\hline
\end{tabular}

Table 5.1. Thioredoxin-related genes up-regulated during starvation/stationary phase. GST, Glutathione $S$-transferase. 
Mutants 1B3, 3B10, 4G11 and 10E4:

5'-CACCGACGT CTCCAAGGA<TN KAN-2>CTCCAAGGA CGGCACACT-3'

Mutant 3B4:

5'- GCGTGAGGC GGTCGAGCC<TN KAN-2>GGTCGAGCC GGATCCGCG-3'

Mutant 10D3:

5'- ACCCATGCC GATCTCAAC <TN KAN-2>GATCTCAAC GAGGTCGCG-3'

Mutant 6E6:

5'- AGAGTGTTC TCCTCGCTCT<TN KAN-2>TCCTCGCTCT GGCAGGCGA-3'

Mutant 4G6:

5'- TGACCGCAC GACCGGCCT<TN KAN-2>CACCCGCCT CACCCTGCG-3'

Figure 5.1. Transposon insertion sites identified in mutants 1B3, 3B10, 4G6, 10E4, 3B4, 10D3, 6E6 and 4G6. The $9 \mathrm{bp}$ (10 bp for 6E6) repeats either side of the insertion site are shown in bold type. 
In conclusion, $R$. erythropolis SQ1 was shown to be a good candidate for a model or test organism for this study, in particular because of its ease of culture and ease of molecular manipulation. It presents a classic starvation/stationary phase survival response, with the associated increase in resistance to various external stresses.

A mutant bank was also generated, that will be available for future screening of other phenotypes, and a method developed to recover transposon flanking sequences. The mutants identified as being deficient in stationary phase/starvation survival had a transposon insertion in genes $u v r B$ (nucleotide excision repair), his $A$ and his $B$ (histidine synthesis), $\operatorname{ser} A$ (serine/glycine synthesis) and a putative thioredoxin (cytochrome $c$ biogenesis). For one of the mutants (mutant 10D3) the transposon insertion was not within a gene but upstream of IMDH (IMP dehydrogenase) and downstream of guaB (guanine synthesis).

Similar genes have been identified in stationary/starvation deficient mutants of other organisms. Some of those genes are involved in amino acid metabolism, such as hprT (hypoxanthine-guanine phosphoribosyl transferase) in Staphylococcus aureus (Watson et al., 1998a), lysE (exporter of lysine and arginine) in Mycobacterium smegmatis (Smeulders et al., 2004) and ilvE (branched-chain amino acid aminotransferase) in Sinorhizobium meliloti (Uhde et al., 1997). Others are cytochrome-associated, e.g. cydC (cytochrome $d$ oxidase) in E. coli (Siegele et al., 1996), cox (cytochrome $c$ oxidase) in Sinorhizobium meliloti (Uhde et al., 1997) and ctaA (haem A synthase, involved in synthesis of cytochromes $a a_{3}$ and $c a a_{3}$ ) in Staphylococcus aureus (Clements et al., 1999b; Watson et al., 1998a). More work should be performed in order to understand the function of these genes in stationary phase/starvation survival in Rhodococcus and also to identify additional implicated genes. 
Few complete genome sequences of rhodococci are available to date. However, it should be noted that the availability of the Rhodococcus RHA1 genome sequence (McLeod et al., 2006) has been invaluable to the interpretation of the short stretches of DNA sequence retrieved from mutants analysed during the course of this project. The only other genome sequence partially available is that of $R$. equi $103 \mathrm{~S}$ (http://www.sanger.ac.uk/Projects/R equi/). R. erythropolis RR4 and R. opacus B4 are currently being sequenced at the National Institute of Technology and Evaluation in Japan (http://www.bio.nite.go.jp/ngac/e/project-e.html). With additional sequence data, it will be possible to use a site-directed mutagenesis strategy and/or microarrays to progress in this field.

Several sigma factors have already been identified in Rhodococcus RHA1 [ $\sigma^{\mathrm{A}}, \sigma^{\mathrm{B}}$; McLeod et al. (2006)], the sequences of which could be used to identify the corresponding genes in R. erythropolis SQ1. A homologue of relA can also be found in Rhodococcus RHA1, the putative protein sequence of which has high identity ( $>93 \%$ ) with that of M. tuberculosis $\mathrm{H} 37 \mathrm{Rv}$, and would therefore be a prime candidate for sitedirected mutagenesis. Until sequencing of $R$. erythropolis RR4 and R. opacus B4 is completed, it should be possible to identify an equivalent gene in $R$. erythropolis SQ1 by careful design of primers for PCR amplification.

The "resuscitation factor" identified in M. tuberculosis could be another target (Mukamolova et al., 1998). Five rpf genes have been identified in M. tuberculosis so far (Mukamolova et al., 2002), and some mutagenesis work has started in M. tuberculosis and Micrococcus luteus (Downing et al., 2004; Mukamolova et al., 2006). Some limited 
studies have also been performed on $R$. rhodochrous (Shleeva et al., 2002; Voloshin et al., 2005). It should be noted that the procedure to generate 'non-culturable' cells of $R$. rhodochrous is the exact opposite of that observed in this study for $R$. erythropolis SQ1, i.e. shaking vs stationary incubation. Comparison of the two species would provide more information on the role of Rpf in rhodococci.

Other genes of interest for site-directed mutagenesis would be $d p s$, encoding a DNAprotecting protein (Gupta \& Chatterji, 2003), and ppk, encoding a polyphosphate kinase (Hirsch \& Elliott, 2002).

All the genes highlighted previously for site-directed mutagenesis would also be suitable for microarray study. This would allow the study of the expression of the stationary phase/starvation genes when cells are used in industrial processes, e.g. when in a resting state or even isolated from polymer-bound cells. 


\title{
References
}

\begin{abstract}
Abbad-Andaloussi, S., Lagnel, C., Warzywoda, M. \& Monot, F. (2003). Multicriteria comparison of resting cell activities of bacterial strains selected for biodesulfurization of petroleum compounds. Enzyme and Microbial Technology 32, 446-454.
\end{abstract}

Adachi, K., Katsuta, A., Matsuda, S., Peng, X., Misawa, N., Shizuri, Y., Kroppenstedt, R. M., Yokota, A. \& Kasai, H. (2007). Smaragdicoccus niigatensis gen. nov., sp. nov., a novel member of the suborder Corynebacterineae. International Journal of Systematic and Evolutionary Microbiology 57, 297-301.

Ainsworth, D. M., Eicker, S. W., Yeagar, A. E., Sweeney, C. R., Viel, L., Tesarowski, D., Lavoie, J. P., Hoffman, A., Paradis, M. R. \& other authors (1998). Associations between physical examination, laboratory, and radiographic findings and outcome and subsequent racing performance of foals with Rhodococcus equi infection: 115 cases (1984-1992). Journal Of The American Veterinary Medical Association 213, $510-515$.

Al-Maghrebi, M. A. \& Benov, L. T. (2001). Polyphosphate accumulation and oxidative DNA damage in superoxide dismutase-deficient Escherichia coli. Free Radical Biology and Medicine 31, 1352-1359.

Alberts, J. F., Engelbrecht, Y., Steyn, P. S., Holzapfel, W. H. \& van Zyl, W. H. (2006). Biological degradation of aflatoxin $\mathrm{B} 1$ by Rhodococcus erythropolis cultures. International Journal of Food Microbiology 109, 121-126.

Alifano, P., Fani, R., Liò, P., Lazcano, A., Bazzicalupo, M., Carlomagno, M. \& Bruni, C. (1996). Histidine biosynthetic pathway and genes: structure, regulation, and evolution. Microbiological Reviews 60, 44-69.

Almirón, M., Link, A., Furlong, D. \& Kolter, R. (1992). A novel DNA-binding protein with regulatory and protective roles in starved Escherichia coli. Genes \& Development 6, 2646-2654.

Altschul, S. F., Madden, T. L., Schaffer, A. A., Zhang, J., Zhang, Z., Miller, W. \& Lipman, D. J. (1997). Gapped BLAST and PSI-BLAST: a new generation of protein database search programs. Nucleic Acids Research 25, 3389-3402.

Altuvia, S., Weinstein-Fischer, D., Zhang, A., Postow, L. \& Storz, G. (1997). A small, stable RNA induced by oxidative stress: role as a pleiotropic regulator and antimutator. Cell 90, 43-53.

Anonymous (1998). Directive on the quality of petrol and diesel fuels. Official Journal of the European Union. 
Anonymous (2007). Proposal for a directive of the European parliament and of the council amending Directive 98/70/EC as regards the specification of petrol, diesel and gas-oil: Commission of the European Communities.

Archer, J. \& Sinskey, A. (1993). The DNA sequence and minimal replicon of the Corynebacterium glutamicum plasmid pSR1: evidence of a common ancestry with plasmids from C. diphtheriae. Journal of General Microbiology 139, 1753-1759.

Arikan, E., Kulkami, M. S., Thomas, D. C. \& Sancar, A. (1986). Sequences of the $E$. coli uvrB gene and protein. Nucleic Acids Research 14, 2637-2650.

Armfield, S. J., Sallis, P. J., Baker, P. B., Bull, A. T. \& Hardman, D. J. (1995). Dehalogenation of haloalkanes by Rhodococcus erythropolis Y2. Biodegradation 6, 237-246.

Ashour, J. \& Hondalus, M. K. (2003). Phenotypic mutants of the intracellular actinomycete Rhodococcus equi created by in vivo Himarl transposon mutagenesis. Journal of Bacteriology 185, 2644-2652.

Atlas, R. M. (1996). Handbook of Microbiological Media, 2nd edn. Boca Raton, FL, London: CRC Press.

Autret, S., Levine, A., Holland, I. B. \& Séror, S. J. (1997). Cell cycle checkpoints in bacteria. Biochimie 79, 549-554.

Avarbock, D., Salem, J., Li, L.-S., Wang, Z.-M. \& Rubin, H. (1999). Cloning and characterization of a bifunctional RelA/SpoT homologue from Mycobacterium tuberculosis. Gene 233, 261-269.

Baker, R. M., Singleton, F. L. \& Hood, M. A. (1983). Effects of nutrient deprivation on Vibrio cholerae. Applied Environmental Microbiology 46, 930-940.

Banba, Hiroyasu, M. \& Natsuko (2002). Process for producing acrylamide using a microbial catalyst having been washed with aqueous acrylic acid solution. United States Patent and Trademark Office, Patent number 7,205,133.

Barona-Gómez, F. \& Hodgson, D. A. (2003). Occurrence of a putative ancient-like isomerase involved in histidine and tryptophan biosynthesis. EMBO reports 4, 296-300.

Begoña Prieto, M., Hidalgo, A., Serra, J. L. \& Llama, M. J. (2002). Degradation of phenol by Rhodococcus erythropolis UPV-1 immobilized on Biolite ${ }^{\circledR}$ in a packed-bed reactor. Journal of Biotechnology 97, 1-11.

Begonja Kolar, A., Hršak, D., Fingler, S., Ćetković, H., Petric, I. \& Kolić, N. U. (2007). PCB-degrading potential of aerobic bacteria enriched from marine sediments. International Biodeterioration \& Biodegradation 60, 16-24.

Bej, A. K., Saul, D. \& Aislabie, J. (2000). Cold-tolerant alkane-degrading Rhodococcus species from Antarctica. Polar Biology 23, 100-105. 
Bell, K. S., Philp, J. C., Christofi, N. \& Aw, D. W. J. (1996). Identification of Rhodococcus equi using the polymerase chain reaction. Letters in Applied Microbiology 23, $72-74$.

Bell, K. S., Philp, J. C., Aw, D. W. J. \& Christofi, N. (1998). The genus Rhodococcus. Journal of Applied Microbiology 85, 195-210.

Bell, K. S., Kuyukina, M. S., Heidbrink, S., Philp, J. C., Aw, D. W. J., Ivshina, I. B. \& Christofi, N. (1999). Identification and environmental detection of Rhodococcus species by $16 \mathrm{~S}$ rDNA-targeted PCR. Journal of Applied Microbiology 87, 472-480.

Benita, Y., Oosting, R. S., Lok, M. C., Wise, M. J. \& Humphery-Smith, I. (2003). Regionalized GC content of template DNA as a predictor of PCR success. Nucleic Acids Research 31, e99.

Berg, D. E., Schmandt, M. A. \& Lowe, J. B. (1983). Specificity of transposon Tn5 insertion. Genetics 105, 813-828.

Berg, J. M., Tymoczko, J. \& Stryer, L. (2002). Biochemistry, 5th edn. New York: W. H. Freeman and Company.

Besnard, V., Federighi, M. \& Cappelier, J. M. (2000). Evidence of viable but nonculturable state in Listeria monocytogenes by direct viable count and CTC-DAPI double staining. Food Microbiology 17, 697-704.

Betts, J. C., Lukey, P. T., Robb, L. C., McAdam, R. A. \& Duncan, K. (2002). Evaluation of a nutrient starvation model of Mycobacterium tuberculosis persistence by gene and protein expression profiling. Molecular Microbiology 43, 717-731.

Bisset, K. \& Moore, F. (1950). Jensenia, a new genus of the actinomycetales. Journal of General Microbiology 4, 280.

Bizet, C., Barreau, C., Harmant, C., Nowakowski, M. \& Pietfroid, A. (1997). Identification of Rhodococcus, Gordona and Dietzia species using carbon source utilization tests ("Biotype-100" strips). Research in Microbiology 148, 799-809.

Boland, C. A. \& Meijer, W. G. (2000). The iron dependent regulatory protein IdeR (DtxR) of Rhodococcus equi. FEMS Microbiology Letters 191, 1-5.

Borole, A. P., Kaufman, E. N., Grossman, M. J., Minak-Bernero, V., Bare, R. \& Lee, M. K. (2002). Comparison of the emulsion characteristics of Rhodococcus erythropolis and Escherichia coli SOXC-5 cells expressing biodesulfurization genes. Biotechnology Progress 18, 88-93.

Borole, A. P., Hamilton, C. Y. \& Miller, K. (2003). Improving enzyme activity and broadening selectivity for biological desulfurization and upgrading of petroleum feedstocks. Knoxville: University of Tennessee.

Boylan, S., Rutherford, A., Thomas, S. \& Price, C. (1992). Activation of Bacillus subtilis transcription factor $\sigma^{\mathrm{B}}$ by a regulatory pathway responsive to stationary-phase signals. Journal of Bacteriology 174, 3695-3706. 
Bozzi, M., Mignogna, G., Stefanini, S., Barra, D., Longhi, C., Valenti, P. \& Chiancone, E. (1997). A novel non-heme iron-binding ferritin related to the DNAbinding proteins of the Dps family in Listeria innocua. Journal of Biological Chemistry 272, 3259-3265.

Bridges, B. A. (1998). The role of DNA damage in stationary phase ('adaptive') mutation. Mutation Research/DNA Repair 408, 1-9.

Briglia, M., Nurmiaho-Lassila, E. L., Vallini, G. \& Salkinoja-Salonen, M. (1990). The survival of the pentachlorophenol-degrading Rhodococcus chlorophenolicus PCP-1 and Flavobacterium sp. in natural soil. Biodegradation 1, 273-281.

Briglia, M., Rainey, F. A., Stackebrandt, E., Schraa, G. \& Salkinoja-Salonen, M. S. (1996). Rhodococcus percolatus sp. nov., a bacterium degrading 2,4,6- trichlorophenol. International Journal of Systematic Bacteriology 46, 23-30.

Buckland, B. C., Drew, S. W., Connors, N. C., Chartrain, M. M., Lee, C., Salmon, P. M., Gbewonyo, K., Zhou, W., Gailliot, P. \& other authors (1999). Microbial conversion of indene to indandiol: a key intermediate in the synthesis of CRIXIVAN. Metabolic Engineering 1, 63-74.

Burrus, V. \& Waldor, M. K. (2003). Control of SXT integration and excision. Journal of Bacteriology 185, 5045-5054.

Butler, J. S., Springer, M. \& Grunberg-Manago, M. (1987). AUU-to-AUG mutation in the initiator codon of the translation initiation factor IF3 abolishes translational autocontrol of its own gene (infC) in vivo. PNAS 84, 4022-4025.

Butler, W. R., Floyd, M. M., Brown, J. M., Toney, S. R., Daneshvar, M. I., Cooksey, R. C., Carr, J., Steigerwalt, A. G. \& Charles, N. (2005). Novel mycolic acid-containing bacteria in the family Segniliparaceae fam. nov., including the genus Segniliparus gen. nov., with descriptions of Segniliparus rotundus sp. nov. and Segniliparus rugosus sp. nov. International Journal of Systematic and Evolutionary Microbiology 55, 1615-1624.

Caetano-Anollés, G. (1993). Amplifying DNA with arbitrary oligonucleotide primers. PCR methods and applications 3, 85-94.

Caro, A., Boltes, K., Letón, P. \& García-Calvo, E. (2007). Dibenzothiophene biodesulfurization in resting cell conditions by aerobic bacteria. Biochemical Engineering Journal 35, 191-197.

Cavalca, L., Colombo, M., Larcher, S., Gigliotti, C., Collina, E. \& Andreoni, V. (2002). Survival and naphthalene-degrading activity of Rhodococcus sp. strain 1BN in soil microcosms. Journal of Applied Microbiology 92, 1058-1065.

Cavin, J. F., Prevost, H., Lin, J., Schmitt, P. \& Divies, C. (1989). Medium for screening Leuconostoc oenos strains defective in malolactic fermentation. Applied and Environmental Microbiology 55, 751-753. 
Čejková, A., Masak, J., Jirků, V., Veselý, M., Pátek, M. \& Nešvera, J. (2005). Potential of Rhodococcus erythropolis as a bioremediation organism. World Journal of Microbiology and Biotechnology 21, 317-321.

Chan, P. F., Foster, S. J., Ingham, E. \& Clements, M. O. (1998). The Staphylococcus aureus alternative sigma factor $\sigma^{\mathrm{B}}$ controls the environmental stress response but not starvation survival or pathogenicity in a mouse abscess model. Journal of Bacteriology 180, 6082-6089.

Chauvaux, S., Chevalier, F., Le Dantec, C., Fayolle, F., Miras, I., Kunst, F. \& Beguin, P. (2001). Cloning of a genetically unstable cytochrome P-450 gene cluster involved in degradation of the pollutant ethyl tert-butyl ether by Rhodococcus ruber. Journal of Bacteriology 183, 6551-6557.

Chen, L. \& Helmann, J. D. (1995). Bacillus subtilis MrgA is a Dps(PexB) homologue: evidence for metalloregulation of an oxidative-stress gene. Molecular Microbiology 18, 295-300.

Chiaramello, A. E. \& Zyskind, J. W. (1990). Coupling of DNA replication to growth rate in Escherichia coli: a possible role for guanosine tetraphosphate. Journal of Bacteriology 172, 2013-2019.

Christofi, N. \& Ivshina, I. B. (2002). Microbial surfactants and their use in field studies of soil remediation. Journal of Applied Microbiology 93, 915-929.

Clements, M. \& Foster, S. (1998). Starvation recovery of Staphylococcus aureus 8325-4. Microbiology 144, 1755-1763.

Clements, M. O., Watson, S. P. \& Foster, S. J. (1999a). Characterization of the major superoxide dismutase of Staphylococcus aureus and its role in starvation survival, stress resistance, and pathogenicity. Journal of Bacteriology 181, 3898-3903.

Clements, M. O., Watson, S. P., Poole, R. K. \& Foster, S. J. (1999b). CtaA of Staphylococcus aureus is required for starvation survival, recovery, and cytochrome biosynthesis. Journal of Bacteriology 181, 501-507.

Coleman, N. V., Nelson, D. R. \& Duxbury, T. (1998). Aerobic biodegradation of hexahydro-1,3,5-trinitro-1,3,5-triazine (RDX) as a nitrogen source by a Rhodococcus sp., strain DN22. Soil Biology and Biochemistry 30, 1159-1167.

Coleman, N. V., Spain, J. C. \& Duxbury, T. (2002). Evidence that RDX biodegradation by Rhodococcus strain DN22 is plasmid-borne and involves a cytochrome p-450. Journal of Applied Microbiology 93, 463-472.

Crosse, A.-M., Greenway, D. L. A. \& England, R. R. (2000). Accumulation of ppGpp and ppGp in Staphylococcus aureus 8325-4 following nutrient starvation. Letters in Applied Microbiology 31, 332-337.

Dabbs, E. R., Gowan, B. \& Andersen, S. J. (1990). Nocardioform arsenic resistance plasmids and construction of Rhodococcus cloning vectors. Plasmid 23, 242-247. 
Dabbs, E. R. (1998). Cloning of genes that have environmental and clinical importance from rhodococci and related bacteria. Antonie van Leeuwenhoek 74, 155-168.

Dabrock, B., Kesseler, M., Averhoff, B. \& Gottschalk, G. (1994). Identification and characterization of a transmissible linear plasmid from Rhodococcus erythropolis BD2 that encodes isopropylbenzene and trichloroethene catabolism. Applied and Environmental Microbiology 60, 853-860.

Danner, H. \& Braun, R. (1999). Biotechnology for the production of commodity chemicals from biomass. Chemical Society Reviews 28, 395-405.

Darwin, K. H., Ehrt, S., Gutierrez-Ramos, J.-C., Weich, N. \& Nathan, C. F. (2003). The proteasome of Mycobacterium tuberculosis is required for resistance to nitric oxide. Science 302, 1963-1966.

Darwin, K. H. \& Nathan, C. F. (2005). Role for nucleotide excision repair in virulence of Mycobacterium tuberculosis. Infection and Immunity 73, 4581-4587.

de Carvalho, C. C. C. R., Cruz, A., Pons, M. N., Pinheiro, H. M., Cabral, J. M. S., da Fonseca, M. M. R., Fernandes, P. \& Ferreira, B. S. (2004). Mycobacterium sp., Rhodococcus erythropolis and Pseudomonas putida behaviour in the presence of organic solvents. Microscopic Research and Techniques 64, 215-222.

de Carvalho, C. C. C. R. \& da Fonseca, M. M. R. (2004). Solvent toxicity in organicaqueous systems analysed by multivariate analysis. Bioprocess and Biosystems Engineering 26, 361-375.

de Carvalho, C. C. C. R. \& da Fonseca, M. M. R. (2005a). The remarkable Rhodococcus erythropolis. Applied Microbiology and Biotechnology 67, 715-726.

de Carvalho, C. C. C. R. \& da Fonseca, M. M. R. (2005b). Degradation of hydrocarbons and alcohols at different temperatures and salinities by Rhodococcus erythropolis DCL14. FEMS Microbiology Ecology 51, 389-399.

DeMaio, J., Zhang, Y., Ko, C., Young, D. B. \& Bishai, W. R. (1996). A stationaryphase stress-response sigma factor from Mycobacterium tuberculosis. PNAS 93, 27902794.

Denis-Larose, C., Labbé, D., Bergeron, H., Jones, A., Greer, C., al-Hawari, J., Grossman, M., Sankey, B. \& Lau, P. (1997). Conservation of plasmid-encoded dibenzothiophene desulfurization genes in several rhodococci. Applied and Environmental Microbiology 63, 2915-2919.

Denome, S., Olson, E. \& Young, K. (1993). Identification and cloning of genes involved in specific desulfurization of dibenzothiophene by Rhodococcus sp. strain IGTS8. Applied and Environmental Microbiology 59, 2837-2843.

Denome, S., Oldfield, C., Nash, L. \& Young, K. (1994). Characterization of the desulfurization genes from Rhodococcus sp. strain IGTS8. Journal of Bacteriology 176, 6707-6716. 
Desomer, J., Dhaese, P. \& Van Montagu, M. (1988). Conjugative transfer of cadmium resistance plasmids in Rhodococcus fascians strains. Journal of Bacteriology 170, 2401-2405.

Desomer, J., Dhaese, P. \& Montagu, M. V. (1990). Transformation of Rhodococcus fascians by high-voltage electroporation and development of $R$. fascians cloning vectors. Applied and Environmental Microbiology 56, 2818-2825.

Di Mattia, E., Grego, S. \& Cacciari, I. (2002). Eco-physiological characterization of soil bacterial populations in different states of growth. Microbial Ecology 43, 34-43.

Dougherty, T. J. \& Pucci, M. J. (1994). Penicillin-binding proteins are regulated by rpoS during transitions in growth states of Escherichia coli. Antimicrobial Agents and Chemotherapy 38, 205-210.

Downing, K. J., Betts, J. C., Young, D. I., McAdam, R. A., Kelly, F., Young, M. \& Mizrahi, V. (2004). Global expression profiling of strains harbouring null mutations reveals that the five rpf-like genes of Mycobacterium tuberculosis show functional redundancy. Tuberculosis $\mathbf{8 4}, 167-179$.

Ducey, T. F. \& Dyer, D. W. (2002). Rapid identification of EZ::TN transposon insertion sites in the genome of Neisseria gonorrhoeae. EPICENTRE Forum 9, 6-7.

Dukan, S. \& Nyström, T. (1999). Oxidative stress defense and deterioration of growtharrested Escherichia coli cells. Journal of Biological Chemistry 274, 26027-26032.

Duncan, M. L., Kalman, S. S., Thomas, S. M. \& Price, C. W. (1987). Gene encoding the 37,000-dalton minor sigma factor of Bacillus subtilis RNA polymerase: isolation, nucleotide sequence, chromosomal locus, and cryptic function. Journal of Bacteriology 169, $771-778$.

Duwat, P., Cesselin, B., Sourice, S. \& Gruss, A. (2000). Lactococcus lactis, a bacterial model for stress responses and survival. International Journal of Food Microbiology 55, 83-86.

Eccleston, M. \& Kelly, D. (1973). Inhibition by L-threonine of aspartokinase as a cause of threonine toxicity to Methylococcus capsulatus. Journal of General Microbiology 75, 223-226.

Eisen, J. A. \& Hanawalt, P. C. (1999). A phylogenomic study of DNA repair genes, proteins, and processes. Mutation Research/DNA Repair 435, 171-213.

Emelyanova, E. V. \& Reshetilov, A. N. (2002). Rhodococcus erythropolis as the receptor of cell-based sensor for 2,4-dinitrophenol detection: effect of 'co-oxidation'. Process Biochemistry 37, 683-692.

Euzéby, J. P. (2007). List of prokaryotic names with standing in nomenclature, accessed on 21 March 2008. http://www.bacterio.cict.fr/index.html

Eymann, C., Homuth, G., Scharf, C. \& Hecker, M. (2002). Bacillus subtilis functional genomics: global characterization of the stringent response by proteome and transcriptome analysis. Journal of Bacteriology 184, 2500-2520. 
Fabianek, R. A., Hennecke, H. \& Thöny-Meyer, L. (1998). The active-site cysteines of the periplasmic thioredoxin-like protein CcmG of Escherichia coli are important but not essential for cytochrome $c$ maturation in vivo. Journal of Bacteriology 180, 19471950.

Fairley, R. A. \& Fairley, N. M. (1999). Rhodococcus equi infection of cats. Veterinary Dermatology 10, 43-46.

Feng, J., Zeng, Y., Ma, C., Cai, X., Zhang, Q., Tong, M., Yu, B. \& Xu, P. (2006). The surfactant Tween 80 enhances biodesulfurization. Applied and Environmental Microbiology 72, 7390-7393.

Fernandes, P. J., Powell, J. A. C. \& Archer, J. A. C. (2001). Construction of Rhodococcus random mutagenesis libraries using Tn5 transposition complexes. Microbiology 147, 2529-2536.

Ferreira, A., O'Byrne, C. P. \& Boor, K. J. (2001). Role of $\sigma^{\mathrm{B}}$ in heat, ethanol, acid, and oxidative stress resistance and during carbon starvation in Listeria monocytogenes. Applied and Environmental Microbiology 67, 4454-4457.

Finnerty, W. R. (1992). The biology and genetics of the genus Rhodococcus. Annual Review of Microbiology 46, 193-218.

Finnerty, W. R. (1994). Biosurfactants in environmental biotechnology. Current Opinion in Biotechnology 5, 291-295.

Folsom, B. R., Schieche, D. R., DiGrazia, P. M., Werner, J. \& Palmer, S. (1999). Microbial desulfurization of alkylated dibenzothiophenes from a hydrodesulfurized middle distillate by Rhodococcus erythropolis I-19. Applied and Environmental Microbiology 65, 4967-4972.

Fothergill-Gilmore, L. A. \& Watson, H. C. (2006). The phosphoglycerate mutases. In Advances in Enzymology and Related Areas of Molecular Biology, pp. 227-313. Edited by M. Alton. New York: John Wiley \& Sons.

Franchini, A. G. \& Egli, T. (2006). Global gene expression in Escherichia coli K-12 during short-term and long-term adaptation to glucose-limited continuous culture conditions. Microbiology 152, 2111-2127.

Freestone, J. F., Hietala, S., Moulton, J. \& Vivrette, S. (1987). Acquired immunodeficiency in a seven-year-old horse. Journal Of The American Veterinary Medical Association 190, 689-691.

Gallant, J., Margason, G. \& Finch, B. (1972). On the turnover of ppGpp in Escherichia coli. Journal of Biological Chemistry 247, 6055-6058.

García, S., Limón, J. C. \& Heredia, N. L. (2001). Cross protection by heat and cold shock to lethal temperatures in Clostridium perfringens. Brazilian Journal of Microbiology 32, 110-112. 
Giard, J.-C., Hartke, A., Flahaut, S., Benachour, A., Boutibonnes, P. \& Auffray, Y. (1996). Starvation-induced multiresistance in Enterococcus faecalis JH2-2. Current Microbiology 32, 264-271.

Giard, J.-C., Hartke, A., Flahaut, S., Boutibonnes, P. \& Auffray, Y. (1997). Glucose starvation response in Enterococcus faecalis JH2-2: survival and protein analysis. Research in Microbiology 148, 27-35.

Giguère, S. \& Prescott, J. F. (1997). Clinical manifestations, diagnosis, treatment, and prevention of Rhodococcus equi infections in foals. Veterinary Microbiology 56, 313334.

Gil, R., Silva, F. J., Pereto, J. \& Moya, A. (2004). Determination of the core of a minimal bacterial gene set. Microbiology and Molecular Biology Reviews 68, 518-537.

Gilan, I., Hadar, Y. \& Sivan, A. (2004). Colonization, biofilm formation and biodegradation of polyethylene by a strain of Rhodococcus ruber. Applied Microbiology and Biotechnology 65, 97-104.

Givskov, M., Eberl, L., Moller, S., Poulsen, L. K. \& Molin, S. (1994). Responses to nutrient starvation in Pseudomonas putida KT2442: analysis of general crossprotection, cell shape, and macromolecular content. Journal of Bacteriology 176, 7-14.

Goethals, K., Vereecke, D., Jaziri, M., Montagu, M. V. \& Holsters, M. (2001). Leafy gall formation by Rhodococcus fascians. Annual Review of Phytopathology 39, 27-52.

Gold, B., Rodriguez, G. M., Marras, S. A. E., Pentecost, M. \& Smith, I. (2001). The Mycobacterium tuberculosis IdeR is a dual functional regulator that controls transcription of genes involved in iron acquisition, iron storage and survival in macrophages. Molecular Microbiology 42, 851-865.

Gomez, E., Santos, V. E., Alcon, A. \& Garcia-Ochoa, F. (2006). Oxygen transport rate on Rhodococcus erythropolis cultures: effect on growth and BDS capability. Chemical Engineering Science 61, 4595-4604.

Goodfellow, M. \& Alderson, G. (1977). The actinomycete-genus Rhodococcus: a home for the 'rhodochrous' complex. Journal of General Microbiology 100, 99-122.

Goodfellow, M. (1989). Rhodococcus. In Bergey's manual of Systematic Bacteriology, 1st edn, vol 4, pp. 2362-2371. Edited by J. G. Holt, N. R. Krieg, P. H. A. Sneath, N. S. Mair, M. E. Sharpe \& S. T. Williams. Baltimore: Williams \& Wilkins.

Goodfellow, M., Alderson, G. \& Chun, J. (1998). Rhodococcal systematics: problems and developments. Antonie van Leeuwenhoek 74, 3-20.

Goodhew, C. F., Wilson, I. B., Hunter, D. J. \& Pettigrew, G. W. (1990). The cellular location and specificity of bacterial cytochrome $c$ peroxidases. Biochemical Journal 271, 707-712.

Goodhue, C. T., Rosazza, J. P. \& Peruzzotti, G. P. (1986). Methods for transformation of organic compounds. In Manual of industrial microbiology and 
biotechnology, pp. 97-121. Edited by A. L. Demain \& N. A. Solomon. Washington, DC: American Society for Microbiology.

Goryshin, I. Y., Miller, J. A., Kil, Y. V., Lanzov, V. A. \& Reznikoff, W. S. (1998). Tn5/IS50 target recognition. PNAS 95, 10716-10721.

Goryshin, I. Y. \& Reznikoff, W. S. (1998). Tn5 in vitro transposition. Journal of Biological Chemistry 273, 7367-7374.

Goswami, M., Shivaraman, N. \& Singh, R. P. (2005). Microbial metabolism of 2chlorophenol, phenol and $\rho$-cresol by Rhodococcus erythropolis M1 in co-culture with Pseudomonas fluorescens P1. Microbiological Research 160, 101-109.

Goulding, C. W., Apostol, M. I., Gleiter, S., Parseghian, A., Bardwell, J., Gennaro, M. \& Eisenberg, D. (2004). Gram-positive DsbE proteins function differently from Gram-negative DsbE homologs: a structure to function analysis of DsbE from Mycobacterium tuberculosis. Journal of Biological Chemistry 279, 3516-3524.

Gray, K. A., Pogrebinsky, O. S., Mrachko, G. T., Xi, L., Monticello, D. J. \& Squires, C. H. (1996). Molecular mechanisms of biocatalytic desulfurization of fossil fuels. Nature Biotechnology 14, 1705-1708.

Guerinik, K. \& Al-Mutawah, Q. (2003). Isolation and characterization of oildesulphurizing bacteria. World Journal of Microbiology and Biotechnology 19, 941945.

Gupta, S., Pandit, S. B., Srinivasan, N. \& Chatterji, D. (2002). Proteomics analysis of carbon-starved Mycobacterium smegmatis: induction of Dps-like protein. Protein Engineering 15, 503-511.

Gupta, S. \& Chatterji, D. (2003). Bimodal protection of DNA by Mycobacterium smegmatis DNA-binding protein from stationary phase cells. Journal of Biological Chemistry 278, 5235-5241.

Haas, F., Mitchell, M. B., Ames, B. N. \& Mitchell, H. K. (1952). A series of histidineless mutants of Neurospora crassa. Genetics 37, 217-226.

Hanahan, D. (1983). Studies on transformation of Escherichia coli with plasmids. Journal of Molecular Biology 166, 557-580.

Hartke, A., Giard, J.-C., Laplace, J.-M. \& Auffray, Y. (1998). Survival of Enterococcus faecalis in an oligotrophic microcosm: changes in morphology, development of general stress resistance, and analysis of protein synthesis. Applied and Environmental Microbiology 64, 4238-4245.

Heald, S. C., Brandão, P. F. B., Hardicre, R. \& Bull, A. T. (2001). Physiology, biochemistry and taxonomy of deep-sea nitrile metabolising Rhodococcus strains. Antonie van Leeuwenhoek 80, 169-183.

Hecker, M., Schumann, W. \& Völker, U. (1996). Heat-shock and general stress response in Bacillus subtilis. Molecular Microbiology 19, 417-428. 
Hecker, M. \& Völker, U. (1998). Non-specific, general and multiple stress resistance of growth-restricted Bacillus subtilis cells by the expression of the $\sigma^{\mathrm{B}}$ regulon. Molecular Microbiology 29, 1129-1136.

Hengge-Aronis, R., Klein, W., Lange, R., Rimmele, M. \& Boos, W. (1991). Trehalose synthesis genes are controlled by the putative sigma factor encoded by rpoS and are involved in stationary-phase thermotolerance in Escherichia coli. Journal of Bacteriology 173, 7918-7924.

Hengge-Aronis, R. (1993). Survival of hunger and stress: the role of $r p o S$ in early stationary phase gene regulation in E. coli. Cell 72, 165-168.

Hengge-Aronis, R. (1996). Back to log phase: $\sigma^{\mathrm{S}}$ as a global regulator in the osmotic control of gene expression in Escherichia coli. Molecular Microbiology 21, 887-893.

Hengge-Aronis, R. (2002a). Stationary phase gene regulation: what makes an Escherichia coli promoter $\sigma^{\mathrm{S}}$-selective? Current Opinion in Microbiology 5, 591-595.

Hengge-Aronis, R. (2002b). Signal transduction and regulatory mechanisms involved in control of the $\sigma^{\mathrm{S}}(\mathrm{RpoS})$ subunit of RNA polymerase. Microbiology and Molecular Biology Reviews 66, 373-395.

Henn-Sax, M., Thoma, R., Schmidt, S., Hennig, M., Kirschner, K. \& Sterner, R. (2002). Two $(\beta \alpha)_{8}$-barrel enzymes of histidine and tryptophan biosynthesis have similar reaction mechanisms and common strategies for protecting their labile substrates. Biochemistry 41, 12032-12042.

Herbert, K. C. \& Foster, S. J. (2001). Starvation survival in Listeria monocytogenes: characterization of the response and the role of known and novel components. Microbiology 147, 2275-2284.

Hernandez, V. \& Bremer, H. (1991). Escherichia coli ppGpp synthetase II activity requires spoT. Journal of Biological Chemistry 266, 5991-5999.

Herron, P. R., Hughes, G., Chandra, G., Fielding, S. \& Dyson, P. J. (2004). Transposon Express, a software application to report the identity of insertions obtained by comprehensive transposon mutagenesis of sequenced genomes: analysis of the preference for in vitro Tn5 transposition into GC-rich DNA. Nucleic Acids Research 32, e113.

Hidalgo, A., Lopategi, A., Prieto, M., Serra, J. \& Llama, M. (2002). Formaldehyde removal in synthetic and industrial wastewater by Rhodococcus erythropolis UPV-1. Applied Microbiology and Biotechnology 58, 260-264.

Hirasawa, K., Ishii, Y., Kobayashi, M., Koizumi, K. \& Maruhashi, K. (2001). Improvement of desulfurization activity in Rhodococcus erythropolis KA2-5-1 by genetic engineering. Bioscience, Biotechnology and Biochemistry 65, 239-246.

Hirsch, M. \& Elliott, T. (2002). Role of ppGpp in rpoS stationary-phase regulation in Escherichia coli. Journal of Bacteriology 184, 5077-5087. 
Holland, H. L., Brown, F. M., Barrett, F., French, J. \& Johnson, D. V. (2003a). Biotransformation of $\beta$-ketosulfides to produce chiral $\beta$-hydroxysulfoxides. Journal of Industrial Microbiology and Biotechnology 30, 292-301.

Holland, H. L., Brown, F. M., Kerridge, A., Pienkos, P. \& Arensdor, J. (2003b). Biotransformation of sulfides by Rhodococcus erythropolis. Journal of Molecular Catalysis B: Enzymatic 22, 219-223.

Holmquist, L. \& Kjelleberg, S. (1993). Changes in viability, respiratory activity and morphology of the marine Vibrio sp. strain S14 during starvation of individual nutrients and subsequent recovery. FEMS Microbiology Ecology 12, 215-223.

Hondalus, M., Diamond, M., Rosenthal, L., Springer, T. \& Mosser, D. (1993). The intracellular bacterium Rhodococcus equi requires Mac-1 to bind to mammalian cells. Infection and Immunity 61, 2919-2929.

Hondalus, M. \& Mosser, D. (1994). Survival and replication of Rhodococcus equi in macrophages. Infection and Immunity 62, 4167-4175.

Hondalus, M. K. (1997). Pathogenesis and virulence of Rhodococcus equi. Veterinary Microbiology 56, 257-268.

Hu, Y. \& Coates, A. R. M. (1999). Transcription of two sigma 70 homologue genes, $\operatorname{sig} A$ and $\operatorname{sig} B$, in stationary-phase Mycobacterium tuberculosis. Journal of Bacteriology 181, 469-476.

Ichihara, A. \& Greenberg, D. M. (1957). Further studies on the pathway of serine formation from carbohydrate. Journal of Biological Chemistry 224, 331-340.

Imlay, J. A. \& Linn, S. (1986). Bimodal pattern of killing of DNA-repair-defective or anoxically grown Escherichia coli by hydrogen peroxide. Journal of Bacteriology 166, 519-527.

Inaoka, T., Matsumura, Y. \& Tsuchido, T. (1998). Molecular cloning and nucleotide sequence of the superoxide dismutase gene and characterization of its product from Bacillus subtilis. Journal of Bacteriology 180, 3697-3703.

Ishikawa, J., Yamashita, A., Mikami, Y., Hoshino, Y., Kurita, H., Hotta, K., Shiba, T. \& Hattori, M. (2004). The complete genomic sequence of Nocardia farcinica IFM 10152. PNAS 101, 14925-14930.

Isono, M. \& Abe, M. (1962). Dehydrogenation of steroids by microorganisms of the genus Mycococcus. United States Patent and Trademark Office, Patent number $3,056,731$.

Ivshina, I. B., Kamenskikh, T. N., Kuyukina, M. S., Ritchkova, M. I., Shadrin, O. A., Ribalka, L. V., Zvereva, L. V. \& Chumakov, O. B. (1994). Methods for Rhodococcus spp. culture conservation and their application for the maintenance of the alkanotrophic rhodococci specialized fund. Mikrobiologyia 63, 118-128. 
Ivshina, I. B., M.V., B., Zvereva, L. V., Ribalka, L. V. \& Yelovikova, E. A. (1995). Phenotypic characterisation of alkanotrophic rhodococci from various ecosystems. Mikrobiologyia 64, 507-513.

Ivshina, I. B., Peshkur, T. A. \& Korobov, V. P. (2002). Efficient uptake of cesium ions by Rhodococcus cells. Microbiology (translated from Mikrobiologiya, Russian) 71, 357-361.

Jadoun, J. \& Bar, R. (1993). Microbial transformations in a cyclodextrin medium. Part 3. Cholesterol oxidation by Rhodococcus erythropolis. Applied Microbiology and Biotechnology 40, 230-240.

Jahns, T. \& Schepp, R. (2001). Isobutylidenediurea degradation by Rhodococcus erythropolis. Biodegradation 12, 317-323.

Jain, S., Bloom, B. R. \& Hondalus, M. K. (2003). Deletion of vapA encoding virulence associated protein A attenuates the intracellular actinomycete Rhodococcus equi. Molecular Microbiology 50, 115-128.

Jasiecki, J. \& Wegrzyn, G. (2006). Transcription start sites in the promoter region of the Escherichia coli pcnB (plasmid copy number) gene coding for poly(A) polymerase I. Plasmid 55, 169-172.

Jenal, U. \& Hengge-Aronis, R. (2003). Regulation by proteolysis in bacterial cells. Current Opinion in Microbiology 6, 163-172.

Jenkins, D. E., Schultz, J. E. \& Matin, A. (1988). Starvation-induced cross protection against heat or $\mathrm{H}_{2} \mathrm{O}_{2}$ challenge in Escherichia coli. Journal of Bacteriology 170, 39103914.

Jishage, M., Kvint, K., Shingler, V. \& Nyström, T. (2002). Regulation of $\sigma$ factor competition by the alarmone ppGpp. Genes \& Development 16, 1260-1270.

Johannes, T. (1996). Chemical versus biochemical conversion: When and how to use biocatalysts. Biotechnology and Bioengineering 52, 290-295.

Kamalakannan, V., Ramachandran, G., Narayanan, S., Vasan, S. K. \& Narayanan, P. R. (2002). Identification of a novel mycobacterial transcriptional regulator and its involvement in growth rate dependence and stringent control. FEMS Microbiology Letters 209, 261-266.

Kampfer, P., Andersson, M. A., Rainey, F. A., Kroppenstedt, R. M. \& SalkinojaSalonen, M. (1999). Williamsia muralis gen. nov., sp. nov., isolated from the indoor environment of a children's day care centre. International Journal of Systematic Bacteriology 49, 681-687.

Kanehisa, M., Goto, S., Hattori, M., Aoki-Kinoshita, K. F., Itoh, M., Kawashima, S., Katayama, T., Araki, M. \& Hirakawa, M. (2006). From genomics to chemical genomics: new developments in KEGG. Nucleic Acids Research 34, D354-357.

Karpenko, E., Vil'danova-Martsishin, R., Scheglova, N., Pirog, T. \& Voloshina, I. (2006). The prospects of using bacteria of the genus Rhodococcus and microbial 
surfactants for the degradation of oil pollutants. Applied Biochemistry and Microbiology 42, 156-159.

Kayser, K. J., Bielaga-Jones, B. A., Jackowski, K., Odusan, O. \& Kilbane, J. J., II (1993). Utilization of organosulfur compounds by axenic and mixed cultures of Rhodococcus rhodochrous IGTS8. Journal of General Microbiology 139, 3123-3129.

Keer, J., Smeulders, M. J., Gray, K. M. \& Williams, H. D. (2000). Mutants of Mycobacterium smegmatis impaired in stationary-phase survival. Microbiology 146, 2209-2217.

Kell, D. B., Kaprelyants, A. S., Weichart, D. H., Harwood, C. R. \& Barer, M. R. (1998). Viability and activity in readily culturable bacteria: a review and discussion of the practical issues. Antonie van Leeuwenhoek 73, 169-187.

Kell, D. B. \& Young, M. (2000). Bacterial dormancy and culturability: the role of autocrine growth factors: Commentary. Current Opinion in Microbiology 3, 238-243.

Kilbane, J. J., II \& Jackowski, K. (1992). Biodesulfurization of water-soluble coalderived material by Rhodococcus rhodochrous IGTS8. Biotechnology and Bioengineering 40, 1107-1114.

Kim, J.-S., Powalla, M., Lang, S., Wagner, F., Lunsdorf, H. \& Wray, V. (1990). Microbial glycolipid production under nitrogen limitation and resting cell condition. Journal of Biotechnology 13, 257-266.

Kishimoto, M., Inui, M., Omasa, T., Katakura, Y., Suga, K.-i. \& Okumura, K. (2000). Efficient production of desulfurizing cells with the aid of expert system. Biochemical Engineering Journal 5, 143-147.

Kitova, A. E., Kuvichkina, T. N., Il'yasov, P. V., Arinbasarova, A. Y. \& Reshetilov, A. N. (2002). A reactor-type biosensor based on Rhodococcus erythropolis HL PM-1 cells for detecting 2,4-dinitrophenol. Applied Biochemistry and Microbiology 38, 500505 .

Kitova, A. E., Kuvichkina, T. N., Arinbasarova, A. Y. \& Reshetilov, A. N. (2004). Degradation of 2,4-dinitrophenol by free and immobilized cells of Rhodococcus erythropolis HL PM-1. Applied Biochemistry and Microbiology 40, 258-261.

Kitts, C. L., Cunningham, D. P. \& Unkefer, P. J. (1994). Isolation of three hexahydro-1,3,5-trinitro-1,3,5-triazine-degrading species of the family Enterobacteriaceae from nitramine explosive-contaminated soil. Applied and Environmental Microbiology 60, 4608-4611.

Kivisaar, M. (2003). Stationary phase mutagenesis: mechanisms that accelerate adaptation of microbial populations under environmental stress. Environmental Microbiology 5, 814-827.

Kjelleberg, S., Albertson, N., Flärdh, K., Holmquist, L., Jouper-Jaan, A., Marouga, R., Östling, J., Svenblad, B. \& Weichart, D. (1993). How do nondifferentiating bacteria adapt to starvation? Antonie van Leeuwenhoek 63, 333-341. 
Kochkina, G. A., Ivanushkina, N. E., Karasev, S. G., Gavrish, E. Y., Gurina, L. V., Evtushenko, L. I., Spirina, E. V., Vorob'eva, E. A., Gilichinskii, D. A. \& other authors (2001). Survival of micromycetes and actinobacteria under conditions of longterm natural cryopreservation. Microbiology (translated from Mikrobiologiya, Russian) 70, 356-364.

Kosjek, B., Stampfer, W., Glueck, S. M., Pogorevc, M., Ellmer, U., Wallner, S. R., Koegl, M. F., Poessl, T. M., Mayer, S. F. \& other authors (2003). Optimization of the organic solvent-stable asymmetric hydrogen transfer system of Rhodococcus ruber DSM 44541: an activity-growth study. Journal of Molecular Catalysis B: Enzymatic 22, $1-6$.

Kozak, M. (1983). Comparison of initiation of protein synthesis in procaryotes, eucaryotes, and organelles. Microbiology and Molecular Biology Reviews 47, 1-45.

Kretschmer, A. \& Wagner, F. (1980a). Influence of hyperbaric oxygen tension on the metabolism of Candida tropicalis and Rhodococcus erythropolis. Applied Microbiology and Biotechnology 10, 51-57.

Kretschmer, A. \& Wagner, F. (1980b). Influence of hyperbaric oxygen tension on growth parameters of Candida tropicalis and Rhodococcus erythropolis. Applied Microbiology and Biotechnology 10, 41-49.

Kullik, I. I. \& Giachino, P. (1997). The alternative sigma factor $\sigma^{\mathrm{B}}$ in Staphylococcus aureus: regulation of the $\operatorname{sig} B$ operon in response to growth phase and heat shock. Archives of Microbiology 167, 151-159.

Kurland, C. G. \& Dong, H. (1996). Bacterial growth inhibition by overproduction of protein. Molecular Microbiology 21, 1-4.

Lamb, D. H. \& Bott, K. F. (1979). Inhibition of Bacillus subtilis growth and sporulation by threonine. Journal of Bacteriology 137, 213-220.

Lang, S. \& Philp, J. C. (1998). Surface-active lipids in rhodococci. Antonie van Leeuwenhoek 74, 59-70.

Lang, S. (2002). Biological amphiphiles (microbial biosurfactants). Current Opinion in Colloid \& Interface Science 7, 12-20.

Lange, R. \& Hengge-Aronis, R. (1994). The cellular concentration of the $\sigma^{\mathrm{S}}$ subunit of RNA-polymerase in Escherichia coli is controlled at the levels of transcription, translation and protein stability. Genes \& Development 8, 1600-1612.

Lazazzera, B. A. (2000). Quorum sensing and starvation: signals for entry into stationary phase. Current Opinion in Microbiology 3, 177-182.

Lee, M., Kim, M. K., Singleton, I., Goodfellow, M. \& Lee, S. T. (2006). Enhanced biodegradation of diesel oil by a newly identified Rhodococcus baikonurensis EN3 in the presence of mycolic acid. Journal of Applied Microbiology 100, 325-333. 
Lessard, P., O'Brien, X., Currie, D. \& Sinskey, A. (2004). pB264, a small, mobilizable, temperature sensitive plasmid from Rhodococcus. BMC Microbiology 4, 15 .

Li, M., Squires, C., Monticello, D. \& Childs, J. (1996). Genetic analysis of the $d s z$ promoter and associated regulatory regions of Rhodococcus erythropolis IGTS8. Journal of Bacteriology 178, 6409-6418.

Li, Y., Kawamura, Y., Fujiwara, N., Naka, T., Liu, H., Huang, X., Kobayashi, K. \& Ezaki, T. (2004). Rothia aeria sp. nov., Rhodococcus baikonurensis sp. nov. and Arthrobacter russicus sp. nov., isolated from air in the Russian space laboratory Mir. International Journal of Systematic and Evolutionary Microbiology 54, 827-835.

Lim, A., Eleuterio, M., Hutter, B., Murugasu-Oei, B. \& Dick, T. (1999). Oxygen depletion-induced dormancy in Mycobacterium bovis BCG. Journal of Bacteriology 181, 2252-2256.

Lin, T.-C., Young, C.-C., Ho, M.-J., Yeh, M.-S., Chou, C.-L., Wei, Y.-H. \& Chang, J.-S. (2005). Characterization of floating activity of indigenous diesel-assimilating bacterial isolates. Journal of Bioscience and Bioengineering 99, 466-472.

Lodish, H., Berk, A., Zipursky, L. S., Matsudaira, P., Baltimore, D. \& Darnell, J. (2000). Molecular Cell Biology, 4th edn. New York: W. H. Freeman and Company.

Madison, L. L. \& Huisman, G. W. (1999). Metabolic engineering of poly(3hydroxyalkanoates): from DNA to plastic. Microbiology and Molecular Biology Reviews 63, 21-53.

Maes, T., Vereecke, D., Ritsema, T., Cornelis, K., Thu, H. N. T., Van Montagu, M., Holsters, M. \& Goethals, K. (2001). The att locus of Rhodococcus fascians strain D188 is essential for full virulence on tobacco through the production of an autoregulatory compound. Molecular Microbiology 42, 13-28.

Makita, Y., de Hoon, M. \& Danchin, A. (2007). Hon-yaku: a biology-driven Bayesian methodology for identifying translation initiation sites in prokaryotes. $B M C$ Bioinformatics 8, 47.

Makrai, L., Takai, S., Tamura, M., Tsukamoto, A., Sekimoto, R., Sasaki, Y., Kakuda, T., Tsubaki, S., Varga, J. \& other authors (2002). Characterization of virulence plasmid types in Rhodococcus equi isolates from foals, pigs, humans and soil in Hungary. Veterinary Microbiology 88, 377-384.

Mangan, M. W. \& Meijer, W. G. (2001). Random insertion mutagenesis of the intracellular pathogen Rhodococcus equi using transposomes. FEMS Microbiology Letters 205, 243-246.

Matin, A. (1991). The molecular basis of carbon-starvation-induced general resistance in Escherichia coli. Molecular Microbiology 5, 3-10.

McDougald, D., Rice, S. A., Weichart, D. \& Kjelleberg, S. (1998). Nonculturability: adaptation or debilitation? FEMS Microbiology Ecology 25, 1-9. 
McFarland, B. L. (1999). Biodesulfurization. Current Opinion in Microbiology 2, 257264.

McLeod, M. P., Warren, R. L., Hsiao, W. W. L., Araki, N., Myhre, M., Fernandes, C., Miyazawa, D., Wong, W., Lillquist, A. L. \& other authors (2006). The complete genome of Rhodococcus sp. RHA1 provides insights into a catabolic powerhouse. PNAS 103, 15582-15587.

Metcalfe, G. \& Brown, M. (1957). Nitrogen fixation by new species of Nocardia. Journal of General Microbiology 17, 567-572.

Minnikin, D. E., Goodfellow, M. \& Collins, M. D. (1977). Lipid composition in the classification and identification of coryneform and related taxa. In Coryneform Bacteria, pp. 85-160. Edited by I. J. Bousfield \& A. G. Callely: Academic Press.

Mitani, Y., Meng, X., Kamagata, Y. \& Tamura, T. (2005). Characterization of LtsA from Rhodococcus erythropolis, an enzyme with glutamine amidotransferase activity. Journal of Bacteriology 187, 2582-2591.

Monticello, D. J. (2000). Biodesulfurization and the upgrading of petroleum distillates. Current Opinion in Biotechnology 11, 540-546.

Morita, R. Y. (1993). Bioavaility of energy and the starvation state. In Starvation in Bacteria, pp. 1-23. Edited by S. Kjelleberg. New York and London: Plenum Publishing Corporation.

Mosser, D. M. \& Hondalus, M. K. (1996). Rhodococcus equi: an emerging opportunistic pathogen. Trends in Microbiology 4, 29-33.

Mukamolova, G. V., Kaprelyants, A. S., Young, D. I., Young, M. \& Kell, D. B. (1998). A bacterial cytokine. PNAS 95, 8916-8921.

Mukamolova, G. V., Turapov, O. A., Young, D. I., Kaprelyants, A. S., Kell, D. B. \& Young, M. (2002). A family of autocrine growth factors in Mycobacterium tuberculosis. Molecular Microbiology 46, 623-635.

Mukamolova, G. V., Murzin, A. G., Salina, E. G., Demina, G. R., Kell, D. B., Kaprelyants, A. S. \& Young, M. (2006). Muralytic activity of Micrococcus luteus Rpf and its relationship to physiological activity in promoting bacterial growth and resuscitation. Molecular Microbiology 59, 84-98.

Murray, D. K. \& Bremer, H. (1996). Control of spoT-dependent ppGpp synthesis and degradation in Escherichia coli. Journal of Molecular Biology 259, 41-57.

Muscatello, G., Anderson, G. A., Gilkerson, J. R. \& Browning, G. F. (2006). Associations between the ecology of virulent Rhodococcus equi and the epidemiology of $R$. equi pneumonia on australian thoroughbred farms. Applied and Environmental Microbiology 72, 6152-6160.

Nagasawa, T. \& Yamada, H. (1990). Application of nitrile converting enzymes for the production of useful compounds. Pure \& Applied Chemistry 62, 1441-1444. 
Nagasawa, T., Shimizu, H. \& Yamada, H. (1993). The superiority of the thirdgeneration catalyst, Rhodococcus rhodochrous J1 nitrile hydratase, for production of acrylamide. Applied Microbiology and Biotechnology 40, 189-195.

Naito, M., Kawamoto, T., Fujino, K., Kobayashi, M., Maruhashi, K. \& Tanaka, A. (2001). Long-term repeated biodesulfurization by immobilized Rhodococcus erythropolis KA2-5-1 cells. Applied Microbiology and Biotechnology 55, 374.

Nester, E. W. \& Montoya, A. L. (1976). An enzyme common to histidine and aromatic amino acid biosynthesis in Bacillus subtilis. Journal of Bacteriology 126, 699-705.

Neu, H. C. \& Gootz, T. D. (1996). Antimicrobial chemotherapy. In Medical Microbiology, 4th edn. Edited by S. Baron. Galveston, TX: The University of Texas Medical Branch.

Noda, K.-i., Watanabe, K. \& Maruhashi, K. (2002). Cloning of a rhodococcal promoter using a transposon for dibenzothiophene biodesulfurization. Biotechnology Letters 24, 1875-1882.

Nyka, W. (1974). Studies on the effect of starvation on mycobacteria. Infection and Immunity 9, 843-850.

Nyström, T., Flärdh, K. \& Kjelleberg, S. (1990). Responses to multiple-nutrient starvation in marine Vibrio sp. strain CCUG 15956. Journal of Bacteriology 172, 70857097.

Nyström, T., Olsson, R. \& Kjelleberg, S. (1992). Survival, stress resistance, and alterations in protein expression in the marine Vibrio sp. strain S14 during starvation for different individual nutrients. Applied and Environmental Microbiology 58, 55-65.

Nyström, T. (1999). Starvation, cessation of growth and bacterial aging. Current Opinion in Microbiology 2, 214-219.

O'Mahony, R., Doran, J., Coffey, L., Cahill, O. J., Black, G. W. \& O'Reilly, C. (2005). Characterisation of the nitrile hydratase gene clusters of Rhodococcus erythropolis strains $\mathrm{AJ} 270$ and $\mathrm{AJ} 300$ and Microbacterium sp. AJ115 indicates horizontal gene transfer and reveals an insertion of IS1166. Antonie van Leeuwenhoek 87, 221-232.

O'Neal, C. R., Gabriel, W. M., Turk, A. K., Libby, S. J., Fang, F. C. \& Spector, M. P. (1994). RpoS is necessary for both the positive and negative regulation of starvation survival genes during phosphate, carbon, and nitrogen starvation in Salmonella typhimurium. Journal of Bacteriology 176, 4610-4616.

O'Toole, G. A. \& Kolter, R. (1998). Initiation of biofilm formation in Pseudomonas fluorescens WCS365 proceeds via multiple, convergent signalling pathways: a genetic analysis. Molecular Microbiology 28, 449-461.

Oberreuter, H., Charzinski, J. \& Scherer, S. (2002). Intraspecific diversity of Brevibacterium linens, Corynebacterium glutamicum and Rhodococcus erythropolis based on partial 16S rDNA sequence analysis and Fourier-transform infrared (FT-IR) spectroscopy. Microbiology 148, 1523-1532. 
Ochman, H., Gerber, A. S. \& Hartl, D. L. (1988). Genetic applications of an inverse polymerase chain reaction. Genetics 120, 621-623.

Ochsner, U. A., Vasil, M. L., Alsabbagh, E., Parvatiyar, K. \& Hassett, D. J. (2000). Role of the Pseudomonas aeruginosa oxyR-recG operon in oxidative stress defense and DNA repair: OxyR-dependent regulation of katB-ankB, ahpB, and ahpC-ahpF. Journal of Bacteriology 182, 4533-4544.

Ohshiro, T., Hirata, T. \& Izumi, Y. (1995). Microbial desulfurization of dibenzothiophene in the presence of hydrocarbon. Applied Microbiology and Biotechnology 44, 249-252.

Ohshiro, T. \& Izumi, Y. (2000). Purification, characterization and crystallization of enzymes for dibenzothiophene desulfurization. Bioseparation 9, 185-188.

Oldfield, C., Pogrebinsky, O., Simmonds, J., Olson, E. \& Kulpa, C. (1997). Elucidation of the metabolic pathway for dibenzothiophene desulphurization by Rhodococcus sp. strain IGTS8 (ATCC 53968). Microbiology 143, 2961-2973.

Omura, S., Ikeda, H., Ishikawa, J., Hanamoto, A., Takahashi, C., Shinose, M., Takahashi, Y., Horikawa, H., Nakazawa, H. \& other authors (2001). Genome sequence of an industrial microorganism Streptomyces avermitilis: deducing the ability of producing secondary metabolites. PNAS 98, 12215-12220.

Overbeck, A. (1891). Zur Kenntnis der Fettfarbstoff —Production bei Spaltpilzen. Nova Acta Leopoldina 55, 399-416.

Overbeeke, A. P. L., Schenkels, P., Secundo, F. \& Jongejan, J. A. (2003). Biocatalytic synthesis of cyclopropanol from cyclopropyl methyl ketone using whole cells of Rhodococcus erythropolis. Journal of Molecular Catalysis B: Enzymatic 21, 5153.

Ozkanca, R. \& Flint, K. P. (1997). Relationship between respiratory enzymes and survival of Escherichia coli under starvation stress in lake water. Journal of Applied Microbiology 82, 301-309.

Paje, M., Neilan, B. \& Couperwhite, I. (1997). A Rhodococcus species that thrives on medium saturated with liquid benzene. Microbiology 143, 2975-2981.

Parish, T., Liu, J., Nikaido, H. \& Stoker, N. G. (1997). A Mycobacterium smegmatis mutant with a defective inositol monophosphate phosphatase gene homolog has altered cell envelope permeability. Journal of Bacteriology 179, 7827-7833.

Patel, R. N., Chu, L. \& Mueller, R. (2003). Diastereoselective microbial reduction of (S)-[3-chloro-2-oxo-1-(phenylmethyl)propyl]carbamic acid, 1,1-dimethylethyl ester. Tetrahedron: Asymmetry 14, 3105-3109.

Patel, S. B., Kilbane, J. J., II \& Webster, D. A. (1997). Biodesulphurisation of dibenzothiophene in hydrophobic media by Rhodococcus sp. strain IGTS8. Journal of Chemical Technology \& Biotechnology 69, 100-106. 
Peressutti, S. R., Alvarez, H. M. \& Pucci, O. H. (2003). Dynamics of hydrocarbondegrading bacteriocenosis of an experimental oil pollution in Patagonian soil. International Biodeterioration \& Biodegradation 52, 21-30.

Perez, M. G. V., Vassilev, T. \& Kemmerly, S. A. (2002). Rhodococcus equi infection in transplant recipients: a case of mistaken identity and review of the literature. Transplant Infectious Disease 4, 52-56.

Philp, J., Kuyukina, M., Ivshina, I., Dunbar, S., Christofi, N., Lang, S. \& Wray, V. (2002). Alkanotrophic Rhodococcus ruber as a biosurfactant producer. Applied Microbiology and Biotechnology 59, 318-324.

Pierpaoli, E. V., Sandmeier, E., Schonfeld, H.-J. \& Christen, P. (1998). Control of the DnaK chaperone cycle by substoichiometric concentrations of the co-chaperones DnaJ and GrpE. Journal of Biological Chemistry 273, 6643-6649.

Pirog, T. P., Shevchuk, T. A., Voloshina, I. N. \& Karpenko, E. V. (2004). Production of surfactants by Rhodococcus erythropolis strain EK-1, grown on hydrophilic and hydrophobic substrates. Applied Biochemistry and Microbiology 40, 470-475.

Pirog, T. P., Shevchuk, T. A., Voloshina, I. N. \& Gregirchak, N. N. (2005). Use of claydite-immobilized oil-oxidizing microbial cells for purification of water from oil. Applied Biochemistry and Microbiology 41, 51-55.

Pizzul, L., Pilar Castillo, M. \& Stenstrom, J. (2006). Characterization of selected actinomycetes degrading polyaromatic hydrocarbons in liquid culture and spiked soil. World Journal of Microbiology and Biotechnology, 1-8.

Prescott, J. F. (1991). Rhodococcus equi: an animal and human pathogen. Clinical Microbiology Reviews 4, 20-34.

Price, M., Alm, E. \& Arkin, A. (2006). The histidine operon is ancient. Journal of Molecular Evolution 62, 807-808.

Pridmore, R. D. (1987). New and versatile cloning vectors with kanamycin-resistance marker. Gene 56, 309-312.

Prieto, M., Hidalgo, A., Rodríguez-Fernández, C., Serra, J. \& Llama, M. (2002). Biodegradation of phenol in synthetic and industrial wastewater by Rhodococcus erythropolis UPV-1 immobilized in an air-stirred reactor with clarifier. Applied Microbiology and Biotechnology 58, 853-860.

Primm, T. P., Andersen, S. J., Mizrahi, V., Avarbock, D., Rubin, H. \& Barry, C. E., III (2000). The stringent response of Mycobacterium tuberculosis is required for long-term survival. Journal of Bacteriology 182, 4889-4898.

Prod'hom, G., Lagier, B., Pelicic, V., Hance, A. J., Gicquel, B. \& Guilhot, C. (1998). A reliable amplification technique for the characterization of genomic DNA sequences flanking insertion sequences. FEMS Microbiology Letters 158, 75-81.

Quan, S. \& Dabbs, E. R. (1993). Nocardioform arsenic resistance plasmid characterization and improved Rhodococcus cloning vectors. Plasmid 29, 74-79. 
Rao, N. \& Kornberg, A. (1996). Inorganic polyphosphate supports resistance and survival of stationary-phase Escherichia coli. Journal of Bacteriology 178, 1394-1400.

Reese, M. G. \& Eeckman, F. H. (1995). Novel neural network algorithms for improved eukaryotic promoter site recognition. In The seventh international genome sequencing and analysis conference. Hyatt Regency, Hilton Head Island, South Carolina, USA.

Ren, J. \& Prescott, J. F. (2003). Analysis of virulence plasmid gene expression of intra-macrophage and in vitro grown Rhodococcus equi ATCC 33701. Veterinary Microbiology 94, 167-182.

Rison, S. C. G., Mattow, J., Jungblut, P. R. \& Stoker, N. G. (2007). Experimental determination of translational starts using peptide mass mapping and tandem mass spectrometry within the proteome of Mycobacterium tuberculosis. Microbiology 153, 521-528.

Roach, P. C. J., Ramsden, D. K., Hughes, J. \& Williams, P. (2003). Development of a conductimetric biosensor using immobilised Rhodococcus ruber whole cells for the detection and quantification of acrylonitrile. Biosensors and Bioelectronics 19, 73-78.

Ron, E. Z. \& Rosenberg, E. (2001). Natural roles of biosurfactants. Minireview. Environmental Microbiology 3, 229-236.

Rowbotham, T. \& Cross, T. (1977). Ecology of Rhodococcus coprophilus and associated actinomycetes in freshwater and agricultural habitats. Journal of General Microbiology 100, 231-240.

Sallam, K. I., Mitani, Y. \& Tamura, T. (2006). Construction of random transposition mutagenesis system in Rhodococcus erythropolis using IS1415. Journal of Biotechnology 121, 13-22.

Sambrook, J., Fritsch, E. F. \& Maniatis, T. (1987). Molecular cloning: a laboratory manual, 2nd edn. Cold Spring Harbor: Cold Spring Harbor Laboratory Press.

Sanin, S. L. (2003). Effect of starvation on resuscitation and the surface characteristics of bacteria. Journal of Environmental Science and Health, Part A 38, 1517 - 1528.

Sanin, S. L., Sanin, F. D. \& Bryers, J. D. (2003). Effect of starvation on the adhesive properties of xenobiotic degrading bacteria. Process Biochemistry 38, 909-914.

Santos, V. E., Galdeano, C., Gomez, E., Alcon, A. \& Garcia-Ochoa, F. (2006). Oxygen uptake rate measurements both by the dynamic method and during the process growth of Rhodococcus erythropolis IGTS8: modelling and difference in results. Biochemical Engineering Journal 32, 198-204.

Sassetti, C. M., Boyd, D. H. \& Rubin, E. J. (2003). Genes required for mycobacterial growth defined by high density mutagenesis. Molecular Microbiology 48, 77-84.

Savill, M. G., Murray, S. R., Scholes, P., Maas, E. W., McCormick, R. E., Moore, E. B. \& Gilpin, B. J. (2001). Application of polymerase chain reaction (PCR) and 
TaqMan $^{\text {TM }}$ PCR techniques to the detection and identification of Rhodococcus coprophilus in faecal samples. Journal of Microbiological Methods 47, 355-368.

Schaffer, A. A., Aravind, L., Madden, T. L., Shavirin, S., Spouge, J. L., Wolf, Y. I., Koonin, E. V. \& Altschul, S. F. (2001). Improving the accuracy of PSI-BLAST protein database searches with composition-based statistics and other refinements. Nucleic Acids Research 29, 2994-3005.

Sekizaki, T., Tanoue, T., Osaki, M., Shimoji, Y., Tsubaki, S. \& Takai, S. (1998). Improved electroporation of Rhodococcus equi. Journal of Veterinary Medical Science 60, 277-279.

Seymour, R. L., Mishra, P. V., Khan, M. A. \& Spector, M. P. (1996). Essential roles of core starvation-stress response loci in carbon-starvation-inducible cross-resistance and hydrogen peroxide-inducible adaptive resistance to oxidative challenge in Salmonella typhimurium. Molecular Microbiology 20, 497-505.

Shkidchenko, A. N., Kobzev, E. N., Petrikevich, S. B., Chugunov, V. A., Kholodenko, V. P. \& Boronin, A. M. (2004). Biodegradation of black oil by microflora of the Bay of Biscay and biopreparations. Process Biochemistry 39, 16711676.

Shleeva, M., Mukamolova, G. V., Young, M., Williams, H. D. \& Kaprelyants, A. S. (2004). Formation of 'non-culturable' cells of Mycobacterium smegmatis in stationary phase in response to growth under suboptimal conditions and their Rpf-mediated resuscitation. Microbiology 150, 1687-1697.

Shleeva, M. O., Bagramyan, K., Telkov, M. V., Mukamolova, G. V., Young, M., Kell, D. B. \& Kaprelyants, A. S. (2002). Formation and resuscitation of 'nonculturable' cells of Rhodococcus rhodochrous and Mycobacterium tuberculosis in prolonged stationary phase. Microbiology 148, 1581-1591.

Siegele, D. A., Almiron, M. \& Kolter, R. (1993). Approaches to the study of survival and death in stationary-phase Escherichia coli. In Starvation in Bacteria, pp. 151-169. Edited by S. Kjelleberg. New York and London: Plenum Publishing Corporation.

Siegele, D. A. \& Kolter, R. (1993). Isolation and characterization of an Escherichia coli mutant defective in resuming growth after starvation. Genes \& Development 7 , 2629-2640.

Siegele, D. A., Imlay, K. R. \& Imlay, J. A. (1996). The stationary-phase-exit defect of cydC ( $\operatorname{sur} B)$ mutants is due to the lack of a functional terminal cytochrome oxidase. Journal of Bacteriology 178, 6091-6096.

Singer, M. E. V. \& Finnerty, W. R. (1988). Construction of an Escherichia coliRhodococcus shuttle vector and plasmid transformation in Rhodococcus spp. Journal of Bacteriology 170, 638-645.

Sledjeski, D. D., Gupta, A. \& Gottesman, S. (1996). The small RNA, DsrA, is essential for the low temperature expression of RpoS during exponential growth in Escherichia coli. EMBO Journal 15, 3993-4000. 
Smeulders, M. J., Keer, J., Speight, R. A. \& Williams, H. D. (1999). Adaptation of Mycobacterium smegmatis to stationary phase. Journal of Bacteriology 181, 270-283.

Smeulders, M. J., Keer, J., Gray, K. M. \& Williams, H. D. (2004). $S$ Nitrosoglutathione cytotoxicity to Mycobacterium smegmatis and its use to isolate stationary phase survival mutants. FEMS Microbiology Letters 239, 221.

Soddell, J. A., Stainsby, F. M., Eales, K. L., Kroppenstedt, R. M., Seviour, R. J. \& Goodfellow, M. (2006). Millisia brevis gen. nov., sp. nov., an actinomycete isolated from activated sludge foam. International Journal of Systematic and Evolutionary Microbiology 56, 739-744.

Sojo, M., Bru, R., Lopez-Molina, D., Garcia-Carmona, F. \& Argüelles, J. C. (1997). Cell-linked and extracellular cholesterol oxidase activities from Rhodococcus erythropolis. Isolation and physiological characterization. Applied Microbiology and Biotechnology 47, 583-589.

Sojo, M., Bru, R. \& García-Carmona, F. (2002). Rhodococcus erythropolis ATCC 25544 as a suitable source of cholesterol oxidase: cell-linked and extracellular enzyme synthesis, purification and concentration. BMC Biotechnology $\mathbf{2}, 3$.

Solovykh, G. N., Korobov, V. P., Karnaukhova, I. V., Lemkina, L. M., Ushakova, E. I., Ustinova, G. M. \& Minakova, V. V. (2004). Lysozyme of the mollusk Unio pictorum and the sensitivity of alkanotrophic rhodococci to its effect. Applied Biochemistry and Microbiology 40, 482-489.

Spector, M. (1998). The starvation-stress response (SSR) of Salmonella. Advances in Microbial Physiology 40, 233-279.

Stackebrandt, E., Rainey, F. A. \& Ward-Rainey, N. L. (1997). Proposal for a new hierarchic classification system, Actinobacteria classis nov. International Journal of Systematic Bacteriology 47, 479-491.

Stothard, P., Van Domselaar, G., Shrivastava, S., Guo, A., O'Neill, B., Cruz, J., Ellison, M. \& Wishart, D. S. (2005). BacMap: an interactive picture atlas of annotated bacterial genomes. Nucleic Acids Research 33, D317-320.

Tai, H. H. \& Sih, C. J. (1970). 3,4-Dihydroxy-9,10-secoandrosta-1,3,5(10)-triene-9, 17dione 4,5-dioxygenase from Nocardia restrictus. I. Isolation of the enzyme and study of its physical and chemical properties. Journal of Biological Chemistry 245, 5062-5071.

Takai, S., Sekizaki, T., Osawa, T., Sugawara, T., Watanabe, Y. \& Tsubaki, T. (1991). Association between a large plasmid and 15- to 17-kilodalton antigens in virulent Rhodococcus equi. Infection and Immunity 59, 4056-4060.

Takai, S., Sasaki, Y., Ikeda, T., Uchida, Y., Tsubaki, S. \& Sekizaki, T. (1994). Virulence of Rhodococcus equi isolates from patients with and without AIDS. Journal Of Clinical Microbiology 32, 457-460.

Takai, S., Imai, Y., Fukunaga, N., Uchida, Y., Kamisawa, K., Sasaki, Y., Tsubaki, S. \& Sekizaki, T. (1995). Identification of virulence-associated antigens and plasmids 
in Rhodococcus equi from patients with AIDS. Journal of Infectious Diseases 172, 1306-1311.

Takai, S., Vigo, G., Ikushima, H., Higuchi, T., Hagiwara, S.-t., Hashikura, S., Sasaki, Y., Tsubaki, S., Anzai, T. \& other authors (1998). Detection of virulent Rhodococcus equi in tracheal aspirate samples by polymerase chain reaction for rapid diagnosis of $R$. equi pneumonia in foals. Veterinary Microbiology 61, 59-69.

Tanaka, Y., Yoshikawa, O., Maruhashi, K. \& Kurane, R. (2002). The $c b s$ mutant strain of Rhodococcus erythropolis KA2-5-1 expresses high levels of Dsz enzymes in the presence of sulfate. Archives of Microbiology 178, 351-357.

Tarasov, A. L., Borzenkov, I. A., Milekhina, E. I., Mysyakina, I. S. \& Belyaev, S. S. (2004). Utilization of $\mathrm{H}_{2} \mathrm{O}_{2}$ as the oxygen source by bacteria of the genera Pseudomonas and Rhodococcus. Microbiology (translated from Mikrobiologiya, Russian) 73, 392-397.

Tark, M., Tover, A., Koorits, L., Tegova, R. \& Kivisaar, M. (2008). Dual role of NER in mutagenesis in Pseudomonas putida. DNA Repair 7, 20-30.

Thomas, S. M., DiCosimo, R. \& Nagarajan, V. (2002). Biocatalysis: applications and potentials for the chemical industry. Trends in Biotechnology 20, 238.

Tilford, P. (1936). Fasciation of sweet peas caused by Phytomonas fascians n. sp. Journal of Agricultural Research 53, 383-394.

Trainor, V. C., Udy, R. K., Bremer, P. J. \& Cook, G. M. (1999). Survival of Streptococcus pyogenes under stress and starvation. FEMS Microbiology Letters 176, 421-428.

Treadway, S. L., Yanagimachi, K. S., Lankenau, E., Lessard, P. A., Stephanopoulos, G. \& Sinskey, A. J. (1999). Isolation and characterization of indene bioconversion genes from Rhodococcus strain I24. Applied Microbiology and Biotechnology 51, 786-793.

Tsukamura, M. (1974). A further numerical taxonomic study of the rhodochrous group. Japanese Journal of Microbiology 18, 37-44.

Turfitt, G. E. (1944). Microbiological agencies in the degradation of steroids: I. The cholesterol-decomposing organisms of soils. Journal of Bacteriology 47, 487-493.

Uhde, C., Schmidt, R., Jording, D., Selbitschka, W. \& Puhler, A. (1997). Stationaryphase mutants of Sinorhizobium meliloti are impaired in stationary-phase survival or in recovery to logarithmic growth. Journal of Bacteriology 179, 6432-6440.

Vales, L. D., Chase, J. W. \& Murphy, J. B. (1979). Orientation of the guanine operon of Escherichia coli K-12 by utilizing strains containing guaB-xse and guaB-upp deletions. Journal of Bacteriology 139, 320-322.

van der Geize, R., Hessels, G. I., van Gerwen, R., van der Meijden, P. \& Dijkhuizen, L. (2001). Unmarked gene deletion mutagenesis of $k s t D$, encoding 3- 
ketosteroid $\Delta 1$-dehydrogenase, in Rhodococcus erythropolis SQ1 using $s a c B$ as counter-selectable marker. FEMS Microbiology Letters 205, 197-202.

van der Werf, M. J., Orru, R. V. A., Overkamp, K. M., Swarts, H. J., Osprian, I., Steinreiber, A., de Bont, J. A. M. \& Faber, K. (1999a). Substrate specificity and stereospecificity of limonene-1,2-epoxide hydrolase from Rhodococcus erythropolis DCL14; an enzyme showing sequential and enantioconvergent substrate conversion. Applied Microbiology and Biotechnology 52, 380-385.

van der Werf, M. J., Swarts, H. J. \& de Bont, J. A. M. (1999b). Rhodococcus erythropolis DCL14 contains a novel degradation pathway for limonene. Applied and Environmental Microbiology 65, 2092-2102.

Van Hamme, J. D., Singh, A. \& Ward, O. P. (2003). Recent advances in petroleum microbiology. Microbiology and Molecular Biology Reviews 67, 503-549.

van Overbeek, L. S., Eberl, L., Givskov, M., Molin, S. \& van Elsas, J. D. (1995). Survival of, and induced stress resistance in, carbon-starved Pseudomonas fluorescens cells residing in soil. Applied and Environmental Microbiology 61, 4202-4208.

van Overbeek, L. S., van Elsas, J. D. \& van Veen, J. A. (1997). Pseudomonas fluorescens Tn5-B20 mutant RA92 responds to carbon limitation in soil. FEMS Microbiology Ecology 24, 57-71.

Vasconcelos, J. A. \& Deneer, H. G. (1994). Expression of superoxide dismutase in Listeria monocytogenes. Applied and Environmental Microbiology 60, 2360-2366.

Veselý, M., Pátek, M., Newvera, J., Cejková, A., Masák, J. \& Jirku, V. (2003). Host-vector system for phenol-degrading Rhodococcus erythropolis based on Corynebacterium plasmids. Applied Microbiology and Biotechnology 61, 523-527.

Vidal, A., Abril, N. \& Pueyo, C. (1998). DNA sequence analysis of spontaneous lacl $^{-\mathrm{d}}$ mutations in $O^{6}$-alkylguanine-DNA alkyltransferase-proficient and -deficient Escherichia coli. Mutagenesis 13, 367-373.

Voelker, U., Voelker, A., Maul, B., Hecker, M., Dufour, A. \& Haldenwang, W. (1995). Separate mechanisms activate $\sigma^{\mathrm{B}}$ of Bacillus subtilis in response to environmental and metabolic stresses. Journal of Bacteriology 177, 3771-3780.

Völker, U., Maul, B. \& Hecker, M. (1999). Expression of the $\sigma^{\mathrm{B}}$-dependent general stress regulon confers multiple stress resistance in Bacillus subtilis. Journal of Bacteriology 181, 3942-3948.

Voloshin, S. A., Shleeva, M. O., Syroeshkin, A. V. \& Kaprelyants, A. S. (2005). The role of intercellular contacts in the initiation of growth and in the development of a transiently nonculturable state by cultures of Rhodococcus rhodochrous grown in poor media. Microbiology (translated from Mikrobiologiya, Russian) 74, 420-427.

Vomberg, A. \& Klinner, U. (2000). Distribution of alkB genes within n-alkanedegrading bacteria. Journal of Applied Microbiology 89, 339-348. 
Wai, S. N., Mizunoe, Y., Takade, A., Kawabata, S.-I. \& Yoshida, S.-I. (1998). Vibrio cholerae O1 strain TSI-4 produces the exopolysaccharide materials that determine colony morphology, stress resistance, and biofilm formation. Applied Environmental Microbiology 64, 3648-3655.

Walsh, D. A. \& Sallach, H. J. (1966). Comparative studies on the pathways for serine biosynthesis in animal tissues. Journal of Biological Chemistry 241, 4068-4076.

Warhurst, A. M. \& Fewson, C. A. (1994). Biotransformations catalysed by the genus Rhodococcus. Critical Reviews in Biotechnology 14, 29-73.

Watanabe, K., Miyashita, M. \& Harayama, S. (2000). Starvation improves survival of bacteria introduced into activated sludge. Applied Environmental Microbiology 66, 3905-3910.

Watson, S., Antonio, M. \& Foster, S. (1998a). Isolation and characterization of Staphylococcus aureus starvation-induced, stationary-phase mutants defective in survival or recovery. Microbiology 144, 3159-3169.

Watson, S. P., Clements, M. O. \& Foster, S. J. (1998b). Characterization of the starvation-survival response of Staphylococcus aureus. Journal of Bacteriology 180, 1750-1758.

Wayne, L. \& Hayes, L. (1996). An in vitro model for sequential study of shiftdown of Mycobacterium tuberculosis through two stages of nonreplicating persistence. Infection and Immunity 64, 2062-2069.

Weber, H., Polen, T., Heuveling, J., Wendisch, V. F. \& Hengge, R. (2005). Genomewide analysis of the general stress response network in Escherichia coli: $\sigma^{\mathrm{S}}$-dependent genes, promoters, and sigma factor selectivity. Journal of Bacteriology 187, 1591-1603.

Weichart, D., Querfurth, N., Dreger, M. \& Hengge-Aronis, R. (2003). Global role for ClpP-containing proteases in stationary-phase adaptation of Escherichia coli. Journal of Bacteriology 185, 115-125.

Wendrich, T. M. \& Marahiel, M. A. (1997). Cloning and characterization of a relA/spoT homologue from Bacillus subtilis. Molecular Microbiology 26, 65-79.

Weyland, H. (1969). Actinomycetes in North Sea and Atlantic Ocean Sediments. Nature 223, 858-858.

WHO (2002). Tuberculosis Fact Sheet No 104, revised March 2007: World Health Organisation.

Wise, A. \& Price, C. (1995). Four additional genes in the $\operatorname{sig} B$ operon of Bacillus subtilis that control activity of the general stress factor $\sigma^{\mathrm{B}}$ in response to environmental signals. Journal of Bacteriology 177, 123-133.

Woolcock, J. B., Farmer, A. M. \& Mutimer, M. D. (1979). Selective medium for Corynebacterium equi isolation. Journal Of Clinical Microbiology 9, 640-642. 
Wu, S., de Lencastre, H. \& Tomasz, A. (1996). Sigma-B, a putative operon encoding alternate sigma factor of Staphylococcus aureus RNA polymerase: molecular cloning and DNA sequencing. Journal of Bacteriology 178, 6036-6042.

Xiao, H., Kalman, M., Ikehara, K., Zemel, S., Glaser, G. \& Cashel, M. (1991). Residual guanosine 3',5'-bispyrophosphate synthetic activity of relA null mutants can be eliminated by spoT null mutations. Journal of Biological Chemistry 266, 5980-5990.

Yagafarova, G. G., Safarov, A. K., Il'ina, E. G., Yagafarov, I. R., Barakhnina, V. B. \& Sukharevich, M. E. (2002). Effect of shale kerogen oxidation products on biodegradation of oil and oil products in soil and water. Applied Biochemistry and Microbiology 38, 441-444.

Yamazaki, Y., Danelishvili, L., Wu, M., MacNab, M. \& Bermudez, L. E. (2006). Mycobacterium avium genes associated with the ability to form a biofilm. Applied and Environmental Microbiology 72, 819-825.

Yang, J. C. (2006). Conjugation of extrachromosomal replicons of Rhodococcus erythropolis AN12, PhD Thesis. Department of Biology, Massachussetts Institute of Technology.

Yang, J. C., Lessard, P. A., Sengupta, N., Windsor, S. D., O'Brien, X. M., Bramucci, M., Tomb, J.-F., Nagarajan, V. \& Sinskey, A. J. (2007a). TraA is required for megaplasmid conjugation in Rhodococcus erythropolis AN12. Plasmid 57, $55-70$.

Yang, J. C., Lessard, P. A. \& Sinskey, A. J. (2007b). Characterization of the mobilization determinants of pAN12, a small replicon from Rhodococcus erythropolis AN12. Plasmid 57, 71-81.

Yuan, Y., Crane, D. D. \& Barry, C. E., III (1996). Stationary phase-associated protein expression in Mycobacterium tuberculosis: function of the mycobacterial alphacrystallin homolog. Journal of Bacteriology 178, 4484-4492.

Zapparoli, G. (2004). Colony dimorphism associated with stress resistance in Oenococcus oeni VP01 cells during stationary growth phase. FEMS Microbiology Letters 239, 261.

Zeller, T. \& Klug, G. (2006). Thioredoxins in bacteria: functions in oxidative stress response and regulation of thioredoxin genes. Naturwissenschaften 93, 259-266.

Zheng, H., Tkachuk-Saad, O. \& Prescott, J. F. (1997). Development of a Rhodococcus equi-Escherichia coli plasmid shuttle vector. Plasmid 38, 180-187.

Zhu, W., Plikaytis, B. B. \& Shinnick, T. M. (2003). Resuscitation factors from mycobacteria: homologs of Micrococcus luteus proteins. Tuberculosis 83, 261-269.

Zink, M. C., Yager, J. A., Prescott, J. F. \& Fernando, M. A. (1987). Electron microscopic investigation of intracellular events after ingestion of Rhodococcus equi by foal alveolar macrophages. Veterinary Microbiology 14, 295-305. 
Zinser, E. R. \& Kolter, R. (1999). Mutations enhancing amino acid catabolism confer a growth advantage in stationary phase. Journal of Bacteriology 181, 5800-5807.

Zinser, E. R. \& Kolter, R. (2004). Escherichia coli evolution during stationary phase. Research in Microbiology 155, 328. 